\title{
ALGORITMOS DE INTEGRAÇÃO EFICIENTES PARA O MÉTODO DOS ELEMENTOS DE CONTORNO TRIDIMENSIONAL
}

Eng $^{\circ}$ VALERIO JUNIOR BITENCOURT DE SOUZA

ORIENTADOR: Prof. Dr. HUMBERTO BREVES CODA 
UNIVERSIDADE DE SÃO PAULO

ESCOLA DE ENGENHARIA DE SÃO CARLOS

DEPARTAMENTO DE ENGENHARIA DE ESTRUTURAS

\section{ALGORITMOS DE INTEGRAÇÃO EFICIENTES PARA O MÉTODO DOS ELEMENTOS DE CONTORNO TRIDIMENSIONAL.}

\section{Eng ${ }^{\circ}$ VALERIO JUNIOR BITENCOURT DE SOUZA}

Dissertação apresentada ao Departamento de Estruturas, Escola de Engenharia de São Carlos, Universidade de São Paulo, como parte dos requisitos para a obtenção do título de Mestre em Engenharia de Estruturas.

ORIENTADOR: Prof. Dr. HUMBERTO BREVES CODA

São Carlos

2001 
"Não percas tempo julgando as pessoas, assim não terás tempo para amá-las."

Madre Teresa de Calcutá. 
Aos meus Pais. 


\section{AGRADECIMENTOS}

A Deus, por me ter dado serenidade e paciência nos momentos mais difíceis de minha vida.

À Coordenadoria de Aperfeiçoamento de Pessoal de Nível Superior (CAPES), pela bolsa de estudo concedida.

Ao Professor Humberto Breves Coda, pela extraordinária orientação e amizade dispensada àminha pessoa.

Ao grupo de Mecânica Computacional: Arthur, Marcelo e Patrick pela amizade e idéias discutidas.

Ao Professor Wilson Sérgio Venturini pelos conhecimentos transmitidos e colaboração em partes deste trabalho.

A todos os professores que contribuíram diretamente para a minha formação profissional e pessoal.

Aos funcionários do Departamento de Engenharia de Estruturas, que de alguma forma colaboraram para a realização deste trabalho. 
À Maria Nadir Minatel e todos os funcionários das bibliotecas pela ajuda na revisão das referências bibliográficas.

Aos amigos de turma: Andrea, Ewerton, Fábio, Luciano, Luis Cláudio, Luiz Paulo, Malu, Renê, Ricardo, Robson, Rogério, Valentim, entre outros, pelos incentivos e momentos de descontração proporcionados.

Aos amigos: Alexandre, Bruno, Cristiano, Fabrício, Felippe, Gesiane, Lorenzo e todos aqueles que não tiveram seus nomes aqui citados, pela colaboração direta ou indireta para a realização deste trabalho.

Finalmente, a minha Família pelo apoio e estímulo, sem os quais este trabalho não teria sido possível. 


\section{SUMÁRIO}

LISTA DE FIGURAS ................................................................................... i

LISTA DE GRÁFICOS .................................................................................. iii

LISTA DE TABELAS ...................................................................................... v

LISTA DE ABREVIATURAS E SIGLAS ........................................................viii

LISTA DE SÍMBOLOS ........................................................................................ix

RESUMO ...............................................................................................................xii

ABSTRACT................................................................................................ xiii

CAPÍTULO 1 - INTRODUÇÃO ......................................................................

CAPÍTULO 2 - REVISÃO BIBLIOGRÁFICA ................................................3

\section{CAPÍTULO 3 - CONCEITOS DE TEORIA DE ELASTICIDADE}

TRIDIMENSIONAL ..................................................................................................7

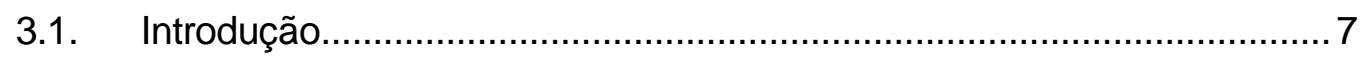

3.2. Notação indicial ......................................................................... 7

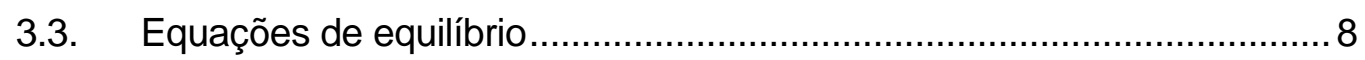

3.4. Relações entre deformação e deslocamento...................................... 10

3.5. Relações entre tensão e deformação.................................................. 11

3.6. Equação de Navier-Cauchy ......................................................... 12

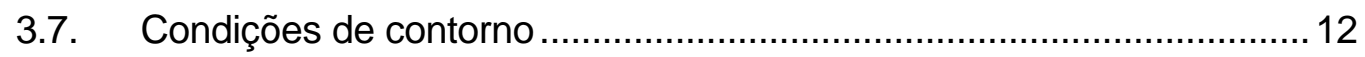

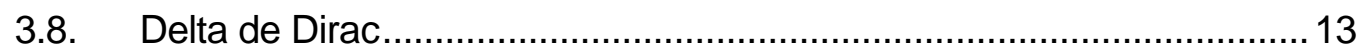


CAPÍTULO 4 - SOLUÇÃO FUNDAMENTAL................................................15

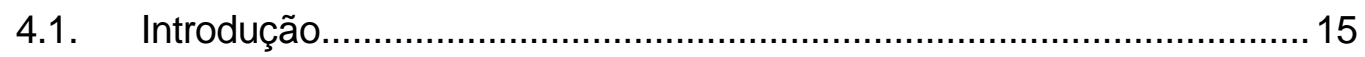

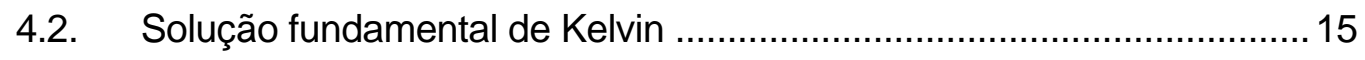

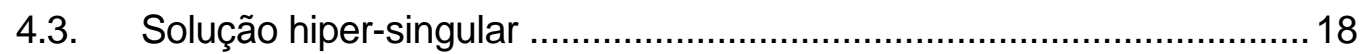

CAPÍTULO 5 - EQUAÇÃO INTEGRAL DE CONTORNO.............................20

5.1. Introdução

5.2. Equação integral para pontos do domínio ............................................. 21

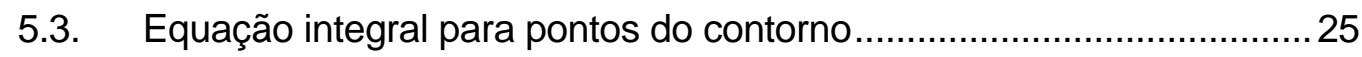

5.4. Equação integral para a solução hiper-singular ................................... 29

CAPÍTULO 6 - MÉTODO DOS ELEMENTOS DE CONTORNO....................31

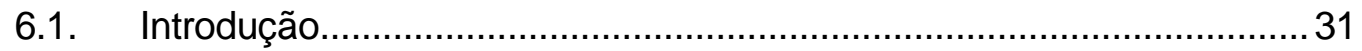

6.2. Equacionamento algébrico ……………………………………......... 31

6.3. Propriedades geométricas dos elementos ........................................... 35

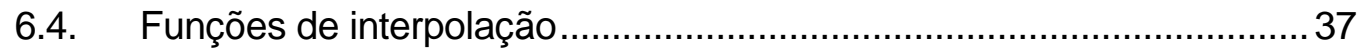

6.4.1. Função de interpolação constante.................................................. 37

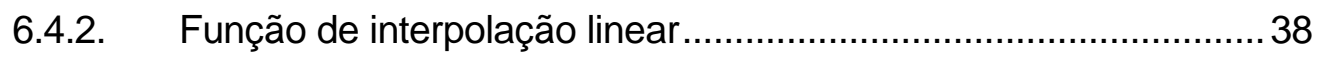

6.4.3. Função de interpolação quadrática ……………………………...... 39

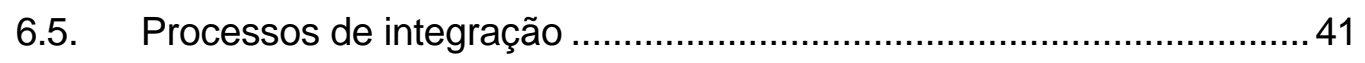

6.5.1. Integral não singular ................................................................ 41

6.5.2. Integral quase singular e quase hiper-singular ............................ 44

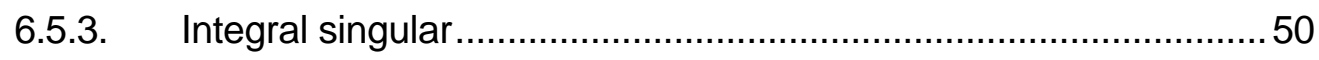

6.6. Montagem do sistema de equações .................................................... 60

6.7. Deslocamentos e tensões em pontos internos ...................................... 62

6.8. Tensões em pontos do contorno …………………………………..... 63 
7.1. Introdução 69

7.2. Exemplo 1 - Sólido tridimensional sob força axial .............................. 70

7.3. Exemplo 2 - Sólido tridimensional sob força transversal ......................78

7.4. Exemplo 3 - Sólido sob força transversal com vários comprimentos ... 89

7.5. Exemplo 4 - Viga bi-apoiada sob força transversal no meio do vão..... 92

7.6. Exemplo 5 - Esfera vazada sob pressão interna.................................. 99

7.7. Exemplo 6 - Casca esférica sob pressão interna............................... 104

7.8. Exemplo 7 - Placa retangular com flexão pura .................................. 106

7.8.1. Placa retangular com curvatura cilíndrica ................................. 106

7.8.2. Placa retangular com curvatura esférica................................. 111

7.8.3. Placa retangular com momentos uniformes em duas bordas..... 113

7.9. Exemplo 8 - Placa quadrada simplesmente apoiada com carga uniformemente distribuída ..................................................................... 115

CAPÍTULO 8 - CONCLUSÕES...................................................................118

ANEXO A

Valor principal de Cauchy para o elemento constante................................. 121

Valor principal de Cauchy para o elemento linear......................................... 123

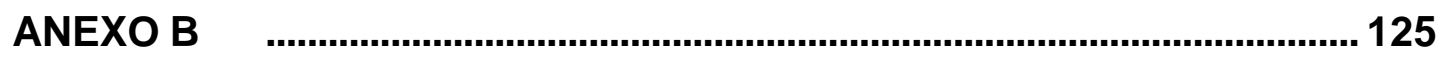

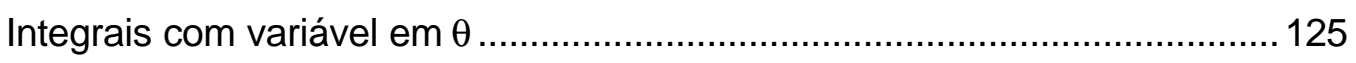

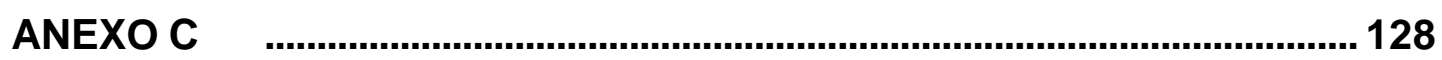

Formulação do MEC para forças concentradas............................................128

REFERÊNCIAS BIBLIOGRÁFICAS..........................................................129

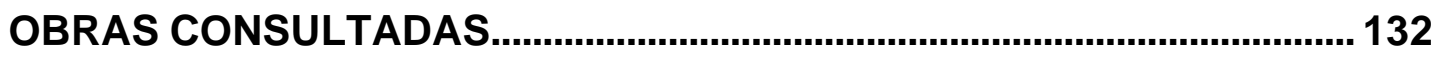

\section{APÊNDICE I}

Soluções analíticas de alguns problemas apresentados....

A. Sólido submetido àforça longitudinal ................................................ I

B. Sólido submetido àforça transversal.................................................. 
C. Viga bi-apoiada submetida a uma carga concentrada no meio do vão. IV

D. Esfera vazada submetida àpressão interna ..................................... V 


\section{LISTA DE FIGURAS}

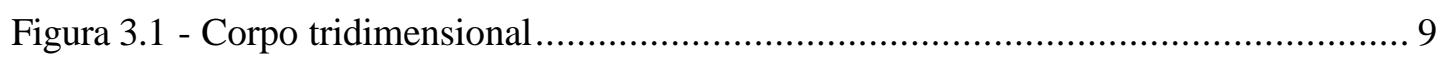

Figura 3.2 - Tensões internas e forças de superfície ........................................................ 10

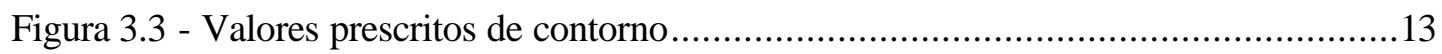

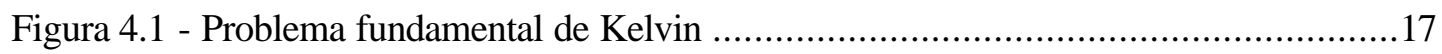

Figura 4.2 - Componentes dos tensores de deslocamentos e forças de superfície ..................18

Figura 5.1 - (a) Contorno expandido no ponto S; (b) Corte AA' ......................................26

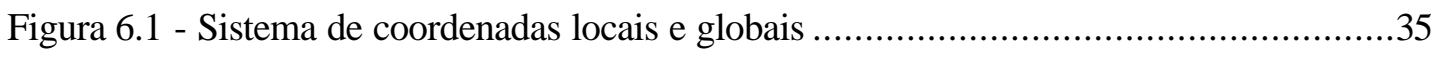

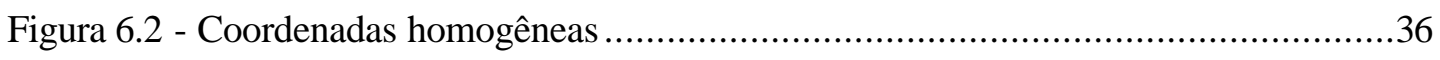

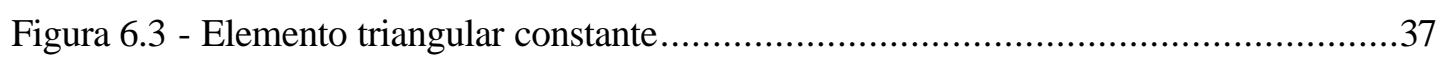

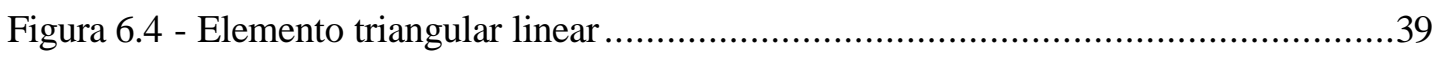

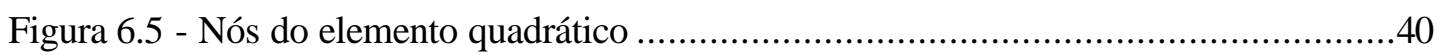

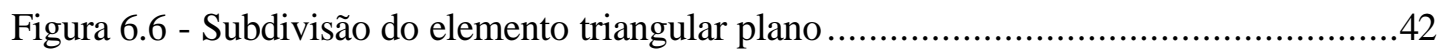

Figura 6.7 - Esquema para integração quase singular para o elemento linear .....................44

Figura 6.8 - Transformação para coordenadas homogêneas..............................................45

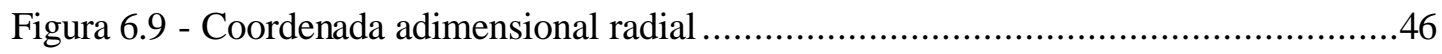

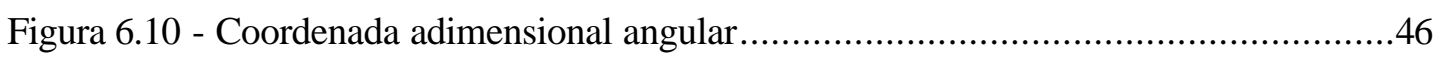

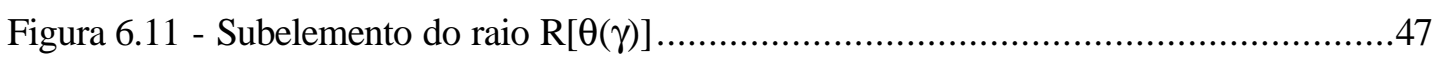

Figura 6.12 - Coordenadas homogêneas generalizadas..................................................49

Figura 6.13 - Divisão do elemento constante para a integral quase singular........................50

Figura 6.14 - Divisão do elemento quadrático para a integral quase singular ......................50

Figura 6.15 - Integral singular sobre o elemento constante .............................................51

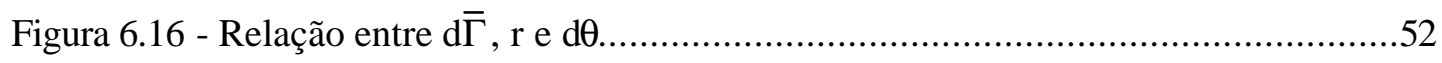

Figura 6.17 - Definição do raio em função do ângulo $\theta$...................................................53

Figura 6.18 - Integral semi-analítica sobre o elemento linear.........................................56

Figura 6.19 - Fluxograma da montagem das equações...................................................61

Figura 6.20 - Análise das tensões em ponto do contorno .................................................64

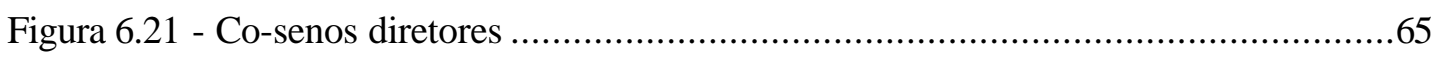


Figura 7.1 - Sólido tridimensional com força distribuída de tração e propriedades físicas e

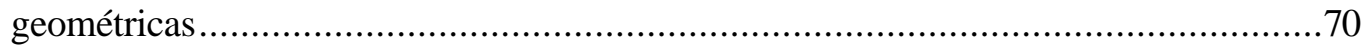

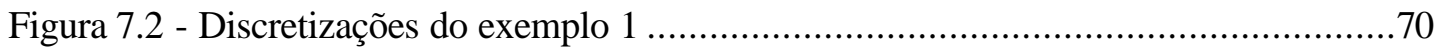

Figura 7.3 - Discretizações da formulação de forças concentradas ....................................76

Figura 7.4 - Sólido tridimensional submetido a uma força transversal - (a) problema clássico

e (b) problema equivalente solucionado pelo MEC …............................................... 78

Figura 7.5 - Discretizações do sólido do exemplo 2 - elemento constante............................79

Figura 7.6 - Discretizações do sólido do exemplo 2 - elemento linear................................82

Figura 7.7 - Discretizações do sólido do exemplo 2 - elemento quadrático ..........................86

Figura 7.8 - Viga bi-apoiada com carregamento concentrado ........................................92

Figura 7.9 - Discretização da viga de $80 \mathrm{~cm}$ indicando carregamento e apoios para o elemento

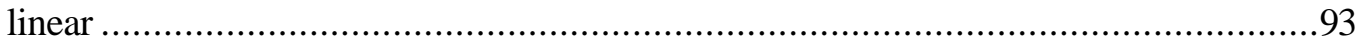

Figura 7.10 - Discretização do sólido do exemplo 4 indicando carregamento e apoios para o

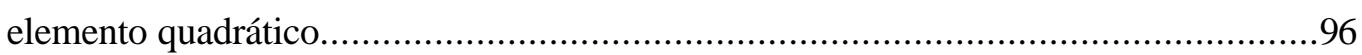

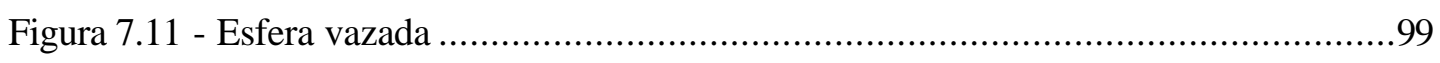

Figura 7.12 - Corte no plano médio da esfera discretizada............................................99

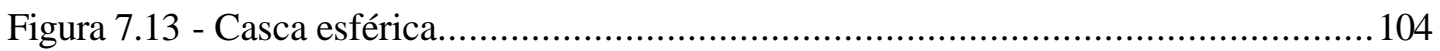

Figura 7.14 - Placa retangular com curvatura cilíndrica - elemento linear........................107

Figura 7.15 - Discretização da placa retangular - L=25cm - malha 8x8 - elemento linear .. 107

Figura 7.16 - Discretização da placa retangular - L=25cm - malha $2 \times 2$ - elemento quadrático

Figura 7.17 - Placa retangular com curvatura esférica - elemento linear ..........................111

Figura 7.18 - Placa retangular com momentos uniformes em duas bordas - elemento linear

Figura 7.19 - Placa quadrada simplesmente apoiada

Figura 7.20 - Discretização da placa quadrada $L=40 \mathrm{~cm}$ - malha $4 \times 4$ - elemento quadrático

Figura A.1 - Valor principal de Cauchy para o elemento constante ...............................121

Figura A.2 - Valor principal de Cauchy para o elemento linear...................................... 123

Figura A.3 - Formulação com forças concentradas ..................................................... 128

Figura I.1 - Sólido elástico sob força longitudinal.......................................................

Figura I.2 - Sólido elástico sob força transversal........................................................

Figura I.3 - Viga bi-apoiada sob flexão simples.................................................... IV

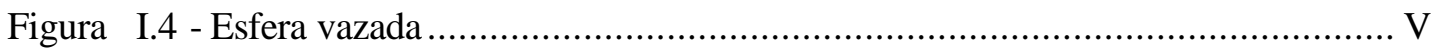




\section{LISTA DE GRÁFICOS}

Gráfico 7.1 - Convergência do elemento constante - exemplo 1.....................................71

Gráfico 7.2 - Estabilidade da solução para o elemento constante - exemplo 1......................71

Gráfico 7.3 - Estabilidade da solução para o elemento linear - exemplo 1 .........................73

Gráfico 7.4 - Estabilidade da solução para o elemento quadrático - exemplo 1 ...................74

Gráfico 7.5 - Convergência da formulação de forças concentradas ....................................77

Gráfico 7.6 - Convergência do elemento constante - exemplo 2 .......................................79

Gráfico 7.7 - Estabilidade da solução para o elemento constante - exemplo 2.....................80

Gráfico 7.8 - Sub-matriz da diagonal principal da matriz H - elemento constante - exemplo 2

Gráfico 7.9 - Estabilidade da solução para o elemento linear - CMEC - exemplo 2.............82

Gráfico 7.10 - Estabilidade da solução para o elemento linear - HMEC - exemplo 2............83

Gráfico 7.11 - Sub-matriz da diagonal principal da matriz H - elemento linear - exemplo 285

Gráfico 7.12 - Estabilidade da solução para o elemento quadrático - exemplo 2 ................87

Gráfico 7.13 - Sub-matriz da diagonal principal da matriz H - elemento quadrático -

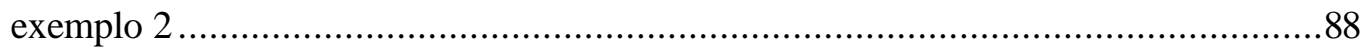

Gráfico 7.14 - Comportamento do deslocamento com a variação do comprimento - elemento linear.

Gráfico 7.15 - Comportamento do deslocamento com a variação do comprimento - elemento quadrático. .91

Gráfico 7.16 - Estabilidade da solução para o elemento linear - exemplo 4. . .93

Gráfico 7.17 - Deslocamentos transversais para pontos sobre o eixo central - viga $80 \mathrm{~cm}$ elemento linear.

Gráfico 7.18 - Deslocamentos transversais para pontos sobre o eixo central - viga $160 \mathrm{~cm}$ elemento linear. .95

Gráfico 7.19 - Estabilidade da solução para o elemento quadrático - exemplo 4 . .96

Gráfico 7.20 - Deslocamentos transversais para pontos sobre o eixo central - viga $80 \mathrm{~cm}$ elemento quadrático. 
Gráfico 7.21 - Deslocamentos transversais para pontos sobre o eixo central - viga 160cm -

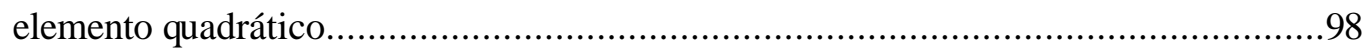

Gráfico 7.22 - Estabilidade do elemento linear para a esfera com relação b/a=1,01 ........... 100

Gráfico 7.23 - Estabilidade do elemento linear para a esfera com relação b/a=2,0 ............ 100

Gráfico 7.24 - Deslocamentos radiais para $\mathrm{r}=\mathrm{a}$ - elemento linear ..................................... 101

Gráfico 7.25 - Tensões circunferenciais para $\mathrm{r}=\mathrm{a}$ - elemento linear .................................. 102

Gráfico 7.26 - Sub-matriz da diagonal principal da matriz H - elemento linear - exemplo 5

Gráfico 7.27 - Convergência da placa retangular com curvatura cilíndrica - L=25cm -

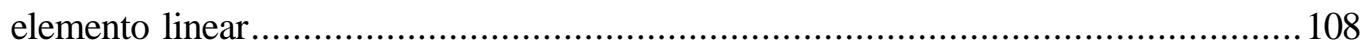

Gráfico 7.28 - Estabilidade da malha 8x8 - L=25cm - elemento linear ........................... 108

Gráfico 7.29 - Estabilidade da malha 2x2 - L=25cm - elemento quadrático …..................110

Gráfico 7.30 - Convergência da placa retangular simplesmente apoiada sob flexão simples -

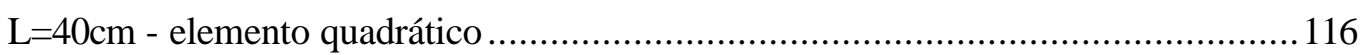

Gráfico 7.31 - Estabilidade da malha 4x4 - L=40cm - elemento quadrático .......................116 


\section{LISTA DE TABELAS}

Tabela 7.1 - Deslocamentos axiais com elemento constante (80 elementos - 240 GL) .........72

Tabela 7.2 - Reações de apoios com elemento constante (80 elementos - 240 GL)...............72

Tabela 7.3 - Deslocamentos axiais com elemento linear (12 elementos - 48 GL) ................73

Tabela 7.4 - Reações de apoios com elemento linear (12 elementos - 48 GL) .....................73

Tabela 7.5 - Deslocamentos axiais com elemento quadrático (12 elementos - 126 GL) .......74

Tabela 7.6 - Reações de apoios com elemento quadrático (12 elementos - 126 GL) .............74

Tabela 7.7 - Número de condicionamento - exemplo 1 - elemento constante .......................75

Tabela 7.8 - Número de condicionamento - exemplo 1 - elemento linear.............................76

Tabela 7.9 - Número de condicionamento - exemplo 1 - elemento quadrático .....................76

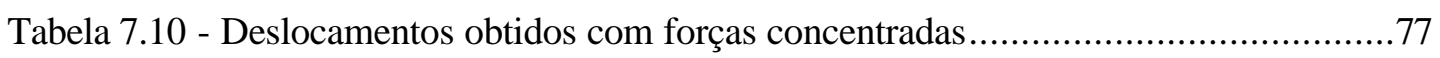

Tabela 7.11 - Número de condicionamento para a formulação de forças concentradas.........77

Tabela 7.12 - Deslocamentos transversais $\left(\mathrm{X}_{2}\right)$ do exemplo 2 com elemento constante - 1296

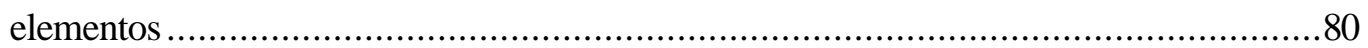

Tabela 7.13 - Número de condicionamento - exemplo 2 - elemento constante ....................80

Tabela 7.14 - Deslocamentos transversais $\left(\mathrm{X}_{2}\right)$ do exemplo 2 com elemento linear - 72

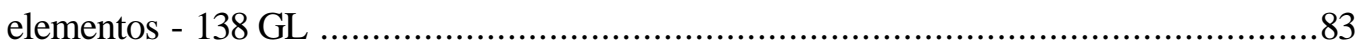

Tabela 7.15 - Número de condicionamento - exemplo 2 - 138 GL - elemento linear ...........83

Tabela 7.16 - Deslocamentos transversais $\left(\mathrm{X}_{2}\right)$ do exemplo 2 com elemento linear - 144

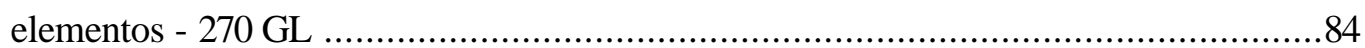

Tabela 7.17 - Número de condicionamento - exemplo 2 - 270 GL - elemento linear ...........84

Tabela 7.18 - Deslocamentos transversais $\left(\mathrm{X}_{2}\right)$ do exemplo 2 com elemento linear - 576

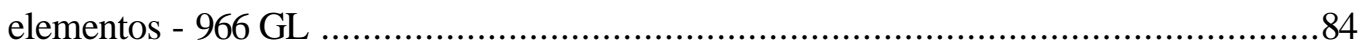

Tabela 7.19 - Número de condicionamento - exemplo 2 - 966 GL - elemento linear ............85

Tabela 7.20 - Deslocamentos transversais $\left(\mathrm{X}_{2}\right)$ do exemplo 2 com elemento quadrático - 36

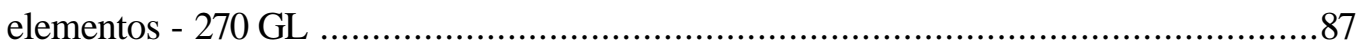

Tabela 7.21 - Número de condicionamento - exemplo 2 - elemento quadrático...................87

Tabela 7.22 - Deslocamentos transversais $\left(\mathrm{X}_{2}\right)$ do exemplo 3 com elemento linear..............89 
Tabela 7.23 - Número de condicionamento - exemplo 3 - elemento linear.. .90

Tabela 7.24 - Deslocamentos transversais $\left(\mathrm{X}_{2}\right)$ do exemplo 3 com elemento quadrático......91

Tabela 7.25 - Número de condicionamento - exemplo 3 - elemento quadrático...................91

Tabela 7.26 - Deslocamentos transversais $\left(\mathrm{X}_{2}\right)$ do exemplo 4 - elemento linear..................94

Tabela 7.27 - Número de condicionamento - exemplo 4 - elemento linear..........................94

Tabela 7.28 - Deslocamentos transversais para pontos sobre o eixo central - viga $80 \mathrm{~cm}$ elemento linear.

Tabela 7.29 - Deslocamentos transversais para pontos sobre o eixo central - viga 160cm elemento linear.

Tabela 7.30 - Deslocamentos transversais $\left(\mathrm{X}_{2}\right)$ do exemplo 4 - elemento quadrático............97

Tabela 7.31 - Número de condicionamento - exemplo 4 - elemento quadrático...................97

Tabela 7.32 - Deslocamentos transversais para pontos sobre o eixo central - viga $80 \mathrm{~cm}$ -

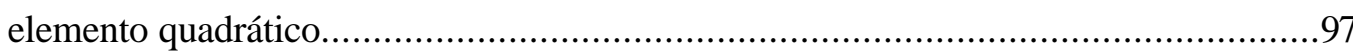

Tabela 7.33 - Deslocamentos transversais para pontos sobre o eixo central - viga $160 \mathrm{~cm}$ -

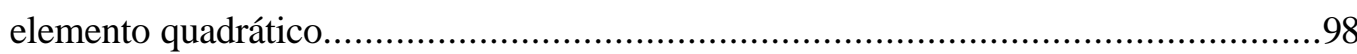

Tabela 7.34 - Deslocamentos radiais para $\mathrm{r}=\mathrm{a}$ - elemento linear........................................ 101

Tabela 7.35 - Tensões circunferenciais para $\mathrm{r}=\mathrm{a}$ - elemento linear ...................................101

Tabela 7.36 - Número de condicionamento - exemplo 5 - elemento linear........................102

Tabela 7.37 - Deslocamentos radiais para r=a - Ponto A - elemento linear ........................ 104

Tabela 7.38 - Deslocamentos radiais para $\mathrm{r}=\mathrm{a}$ - Ponto B - elemento linear.........................105

Tabela 7.39 - Tensões circunferenciais para $\mathrm{r}=\mathrm{a}$ - Ponto A - elemento linear ......................105

Tabela 7.40 - Tensões circunferenciais para $\mathrm{r}=\mathrm{a}$ - Ponto B - elemento linear ...................... 105

Tabela 7.41 - Número de condicionamento - exemplo 6 - elemento linear.......................... 106

Tabela 7.42 - Deslocamentos em placas retangulares com curvatura cilíndrica - elemento linear.....

Tabela 7.43 - Número de condicionamento - curvatura cilíndrica - elemento linear....

Tabela 7.44 - Deslocamentos em placas retangulares com curvatura cilíndrica - elemento quadrático

Tabela 7.45 - Número de condicionamento - curvatura cilíndrica - elemento quadrático ...110

Tabela 7.46 - Deslocamentos em placas retangulares com curvatura esférica - elemento linear.

Tabela 7.47 - Número de condicionamento - curvatura esférica - elemento linear. 112

Tabela 7.48 - Deslocamentos em placas retangulares com curvatura esférica - elemento quadrático

Tabela 7.49 - Número de condicionamento - curvatura esférica - elemento quadrático 
Tabela 7.50 - Deslocamentos em placas retangulares com momentos uniformes em duas

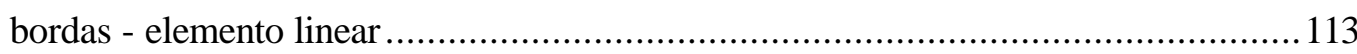

Tabela 7.51 - Número de condicionamento - placas retangulares com momentos uniformes

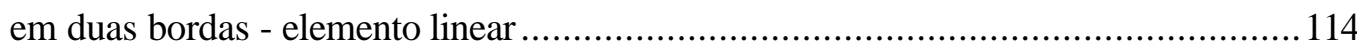

Tabela 7.52 - Deslocamentos em placas retangulares com momentos uniformes em duas bordas - elemento quadrático.

Tabela 7.53 - Número de condicionamento - placas retangulares com momentos uniformes em duas bordas - elemento quadrático. 114

Tabela 7.54 - Comparação dos fatores w(100D/q $\left.H^{+}\right)$- elemento quadrático........................117

Tabela 7.55 - Número de condicionamento - exemplo 8 - elemento quadrático..................117 


\section{LISTA DE ABREVIATURAS E SIGLAS}

$\begin{array}{ll}\text { MEC } & \text { Método dos Elementos de Contorno } \\ \text { MEF } & \text { Método dos Elementos Finitos } \\ \text { MDF } & \text { Método das Diferenças Finitas } \\ \text { 2D } & \text { Bidimensional } \\ \text { 3D } & \text { Tridimensional } \\ \text { CMEC } & \text { Método dos Elementos de Contorno Comum } \\ \text { HMEC } & \text { Método dos Elementos de Contorno Híbrido } \\ \text { ISA } & \text { Integral Singular Calculada Analiticamente } \\ \text { ISSA } & \text { Integral Singular Calculada Semi-Analiticamente } \\ \text { ISQG } & \text { Integral Quase Singular Calculada Numericamente Com } \\ & \text { Quadratura de Gauss } \\ \text { FC } & \text { Formulação com forças concentradas } \\ \text { GL } & \text { Graus de liberdade }\end{array}$




\section{LISTA DE SÍMBOLOS}

\begin{tabular}{|c|c|}
\hline$x_{i}$ & Coordenada cartesiana na direção i \\
\hline $\mathrm{u}_{\mathrm{i}}$ & Deslocamento na direção i \\
\hline $\mathrm{p}_{\mathrm{i}}$ & Força de superfície na direção i \\
\hline$\delta_{i j}$ & Delta de Kronecker \\
\hline$\delta(s, q)$ & Delta de Dirac \\
\hline$\sigma_{i j}$ & Componentes do tensor de tensões \\
\hline$b_{i}$ & Componentes do vetor de forças volumétricas \\
\hline$\Gamma$ & Contorno \\
\hline$\Omega$ & Domínio \\
\hline $\bar{p}_{i}$ & Componentes de força de superfície local ou prescrita \\
\hline $\bar{u}_{i}$ & Componentes de deslocamento local ou prescrito \\
\hline $\mathrm{n}_{\mathrm{j}}$ & Componentes do vetor normal na direção j \\
\hline$\varepsilon_{\mathrm{ij}}$ & Componentes do tensor de deformações \\
\hline $\mathrm{E}$ & Módulo de elasticidade longitudinal \\
\hline G & Módulo de elasticidade transversal \\
\hline$v$ & Coeficiente de Poisson \\
\hline$u_{i j}^{*}$ & Solução fundamental para deslocamentos \\
\hline$\varepsilon^{*}$ & Tensor das deformações do problema fundamental \\
\hline$\sigma^{*}$ & Tensor das tensões do problema fundamental \\
\hline$P_{i j}^{*}$ & Solução fundamental para força de superfície \\
\hline $\mathrm{u}_{\mathrm{ij}, \mathrm{k}}^{*}$ & Derivada da solução fundamental para deslocamentos \\
\hline$P_{i j, k}^{*}$ & Derivada da solução fundamental para força de superfície \\
\hline s & Ponto fonte onde a força unitária é aplicada \\
\hline$q$ & Ponto onde será avaliada a reação das forças unitárias \\
\hline r & Distância entre os pontos s e q \\
\hline$r_{i}$ & Componente cartesiana de $r$ na direção i \\
\hline
\end{tabular}




\begin{tabular}{|c|c|}
\hline$r_{, i}$ & Diferenciação de r na direção i \\
\hline$e_{i}$ & Vetor unitário na direção i \\
\hline$D_{\text {kij }}^{*}$ & Tensor derivado do tensor de deslocamentos fundamentais \\
\hline$S_{\text {kij }}^{*}$ & Tensor derivado do tensor de forças de superfície fundamentais \\
\hline$\varepsilon$ & Raio infinitesimal \\
\hline$c_{i j}$ & Termo livre \\
\hline$\Psi_{\mathrm{k}}$ & Funções interpoladoras \\
\hline$X_{i}^{k}$ & Coordenadas cartesianas dos nós do elemento \\
\hline$\Phi$ & Função interpoladora do elemento \\
\hline$\Phi_{c}$ & Função interpoladora da célula \\
\hline$U^{n}$ & Deslocamentos nodais do elemento \\
\hline$P^{n}$ & Forças de superfície nodais do elemento \\
\hline$B^{n}$ & Forças volumétricas nodais da célula \\
\hline$H$ & $\begin{array}{l}\text { Matriz do método dos elementos de contorno que multiplica o vetor } \\
\text { de deslocamentos }\end{array}$ \\
\hline$G$ & $\begin{array}{l}\text { Matriz do método dos elementos de contorno que multiplica o vetor } \\
\text { de forças de superfície }\end{array}$ \\
\hline$\underset{\sim}{D}$ & $\begin{array}{l}\text { Matriz do método dos elementos de contorno que multiplica o vetor } \\
\text { de forças volumétricas }\end{array}$ \\
\hline C & Matriz dos termos livres \\
\hline$\underset{\sim}{A}$ & $\begin{array}{l}\text { Matriz do sistema de equações do MEC, cujas colunas } \\
\text { correspondem a valores incógnitos }\end{array}$ \\
\hline$X$ & Vetor que contém as incógnitas do sistema de equações do MEC \\
\hline$\underset{\sim}{F}$ & Vetor que possui apenas valores conhecidos \\
\hline$\underset{\sim}{J}$ e $\underset{\sim}{G}$ & Matrizes Jacobianas de transformação \\
\hline$\xi_{\mathrm{i}}$ & Coordenada homogênea na direção i \\
\hline A & Área do elemento \\
\hline $\mathrm{w}_{\mathrm{i}}$ & Peso da quadratura de Gauss ou Hammer \\
\hline $\mathrm{N}$ & Subdivisão do lado do elemento \\
\hline L & Lado do elemento \\
\hline NS & Número de subelementos \\
\hline$\rho$ & Raio adimensional \\
\hline
\end{tabular}




\begin{tabular}{|c|c|}
\hline $\operatorname{ig} \theta$ & Número de pontos de Gauss para variação angular \\
\hline igr & Número de pontos de Gauss para variação radial \\
\hline $\mathrm{W}_{\mathrm{i} \theta}$ & Peso dos pontos de Gauss para a variação angular \\
\hline $\mathrm{w}_{\mathrm{ir}}$ & Peso dos pontos de Gauss para a variação radial \\
\hline $\bar{U}$ & Vetor de deslocamentos nodais nas coordenadas locais \\
\hline $\bar{P}$ & Vetor de forças de superfície nodais nas coordenadas locais \\
\hline$T$ & Matriz de transformação entre os eixos global e local \\
\hline$x$ & Vetor de coordenadas cartesianas locais \\
\hline $\bar{\varepsilon}_{i j}$ & Componentes do tensor de deformações locais \\
\hline $\bar{\sigma}_{i j}$ & Componentes do tensor de tensões locais \\
\hline & Fator de colocação do ponto fora \\
\hline
\end{tabular}




\section{RESUMO}

SOUZA, V. J. B. Algoritmos de integração eficientes para o método de elementos de contorno tridimensional. São Carlos, 2001. Dissertação (Mestrado) - Escola de Engenharia de São Carlos, Universidade de São Paulo.

Neste trabalho é analisado o problema elástico tridimensional através do método dos elementos de contorno empregando a solução fundamental de Kelvin. São utilizadas duas formulações principais: a formulação clássica e a formulação hiper-singular. A primeira utiliza a solução fundamental de Kelvin clássica e a segunda aplica uma derivada direcional da solução fundamental de Kelvin. O contorno é discretizado utilizando-se elemento triangular plano com aproximações constante, linear e quadrática. As integrais singulares são desenvolvidas analiticamente para o elemento constante, e semi-analiticamente para os elementos linear e quadrático. São apresentadas técnicas de integração de contorno considerando-se a eficiência e a precisão para a integral quase singular. São apresentados vários exemplos numéricos, inclusive problemas esbeltos, e seus resultados são comparados com valores conhecidos pela teoria de elasticidade, ou ainda, comparados com valores disponíveis na literatura.

Palavras-chave: elementos de contorno - técnicas de integração - elasticidade tridimensional. 


\begin{abstract}
SOUZA, V. J. B. Efficient integration algorithms for the three-dimensional boundary element method. São Carlos, 2001. Dissertação (Mestrado) - Escola de Engenharia de São Carlos, Universidade de São Paulo.

In this work the three-dimensional elastic problem is analyzed by the boundary element method using the Kelvin fundamental solution. Two main formulations are applied. The first one uses the classical Kelvin fundamental solution and the other, hyper-singular, uses a derivative of the Kelvin fundamental solution. The boundary is discretized by flat triangular elements with constant, linear and quadratic approximations. The singular integrals are analytically developed for constant elements, while for linear and quadratic elements a semi-analytical process is employed. Different techniques to perform quasi-singular boundary integrals are presented and their efficiency and accuracy are compared. Several numerical examples are presented, including slender problems. The results are compared with known solutions given by the theory of elasticity, or with other results found in the literature.
\end{abstract}

Keywords: boundary elements - integration techniques - three-dimensional elasticity. 


\section{CAPÍTULO 1}

\section{INTRODUÇÃO}

Atualmente já é possível resolver problemas elásticos tridimensionais (3D) e bidimensionais (2D) por meio do Método dos Elementos de Contorno (MEC) com extrema agilidade e precisão. Porém, tem-se encontrado enormes dificuldades quando o problema se trata de peças esbeltas, pois as matrizes geradas pelo método tornam-se pouco consistentes. Isto pode ser observado quando se tenta calcular uma placa fina tridimensional com uma solução fundamental para sólidos 3D.

Para que se consiga resolver os problemas de peças esbeltas (3D ou 2D) é necessário que se conheçam as limitações que envolvem o método, e propor novas técnicas para suplantar essas limitações.

Este trabalho insere-se no contexto de buscar alternativas para a melhoria das características numéricas das matrizes do método dos elementos de contorno com o intuito de melhorar o comportamento das soluções de peças esbeltas modeladas pelo MEC 3D, válidas também para formulações bidimensionais.

Neste trabalho também se propõem novas técnicas de integração quase singulares gerais e precisas para elementos de contorno tridimensionais.

Além disso, propõem-se funções ponderadoras alternativas no intuito de melhorar o comportamento numérico das matrizes do MEC, a saber, funções hipersingulares derivadas da própria solução fundamental de Kelvin.

A geração de matrizes consistentes deverá sanar problemas encontrados na resolução de peças esbeltas, placas e cascas delgadas por meio do MEC 3D. 
Apresenta-se no capítulo 2 uma revisão bibliográfica que mostra a evolução do Método dos Elementos de Contorno, com a finalidade de comparar os resultados numéricos de problemas resolvidos nesta pesquisa com soluções obtidas por outros pesquisadores. A revisão bibliográfica também foi realizada com o intuito de solidificar conceitos e tomar conhecimento de outras alternativas para o método, que não seja a usual, principalmente quando se trata da integração quase singular.

No capítulo 3 são apresentados alguns fundamentos de elasticidade tridimensional assim como a notação utilizada neste trabalho.

O capítulo 4 trata da solução fundamental de Kelvin, utilizada para o problema elástico tridimensional. Também é apresentada uma nova formulação, que utiliza soluções hiper-singulares, cujas expressões são obtidas através da diferenciação da solução fundamental de Kelvin em uma direção qualquer.

As equações integrais de contorno, que relacionam deslocamentos de um ponto qualquer do domínio com deslocamentos e esforços no contorno de um corpo tridimensional, são apresentadas no capítulo 5.

Para utilizar as equações integrais no Método dos Elementos de Contorno torna-se necessário discretizar o contorno em elementos, com a finalidade de transformá-las em equações algébricas para serem resolvidas numericamente. Para tal finalidade foi elaborado o capítulo 6 que trata do equacionamento algébrico, das propriedades geométricas dos elementos, das funções de interpolação e principalmente dos processos de integração empregados.

Com a finalidade de analisar a formulação apresentada, são resolvidas várias aplicações numéricas no capítulo 7, comparando-se os resultados numéricos com os obtidos através da teoria da elasticidade e por outros pesquisadores. No capítulo 8 são apresentadas algumas discussões com a finalidade de concluir o presente trabalho.

Os códigos computacionais são desenvolvidos na linguagem Fortran e compilados no ambiente Fortran PowerStation 4.0 da Microsoft. O processamento dos exemplos foi realizado em um micro computador PC utilizando um processador Pentium III 500MHz com 128MB de memória RAM. 


\section{CAPÍTULO 2}

\section{REVISÃO BIBLIOGRÁFICA}

A teoria da elasticidade tem sido bastante usada e desenvolvida ao longo da história, e isto se deve ao fato de que era preciso obter soluções analíticas para vários problemas da mecânica dos sólidos. Infelizmente tais soluções só são possíveis para um número limitado de problemas, além de normalmente se adotar hipóteses simplificadoras para se aplicar os conceitos da teoria da elasticidade tradicional.

Dessa forma era preciso encontrar soluções cujos valores eram aproximados, surgindo, assim, as chamadas técnicas aproximadas, como, por exemplo, aproximações por séries de Fourier. Tais técnicas foram se desenvolvendo e deram origem aos métodos numéricos, esses métodos baseiam-se em soluções que não são obtidas analiticamente, e sim com aproximações numéricas das equações diferenciais que governam os problemas da engenharia.

Os primeiros métodos numéricos formulados em bases matemáticas foram 0 Método das Diferenças Finitas (MDF) e o Método dos Elementos Finitos (MEF). Com o avanço da tecnologia, principalmente com o advento dos computadores, esses métodos foram se desenvolvendo numa velocidade extraordinária. Esses métodos aproximam a equação diferencial que rege o problema a ser analisado, por meio de um sistema de equações que utiliza valores de domínio e de contorno, sendo por isso também chamados de métodos de domínio. Para se obter uma resposta aceitável, é necessária uma grande discretização de todo o domínio. Dessa forma, gera-se um sistema de equações com um grande número de variáveis. 
Devido ànecessidade de técnicas que pudessem diminuir a discretização do problema, conseqüentemente o número de variáveis do sistema de equações a ser resolvido, surgiram as técnicas de contorno, as quais consistem em aproximar o problema analisado apenas em termos de valores de contorno. Tais técnicas têm como ponto de partida as equações integrais.

O mais antigo problema resolvido desta forma de que se tem notícia, é o problema da tautócrona ("tempo igual"), propriedade esta utilizada na formulação de um pêndulo isócrono. Esta solução foi publicada em 1823 e deve-se ao matemático norueguês Niels Henrik Abel ${ }^{1}$. Vários matemáticos depois de Abel utilizaram esta técnica para resolver outros problemas físicos, até culminar com a primeira teoria clássica das equações integrais desenvolvida por Fredholm (1903)².

Por volta dos anos cinqüenta, surgiram vários trabalhos neste contexto e que muito contribuíram para o desenvolvimento dos métodos de contorno, principalmente de autores russos como Muskhelishvili (1953), Mikhlin (1957), Kupradze (1965) e Smirnov (1964) apud Brebbia et al. (1984), dos quais se destaca o trabalho de Kupradze, com o qual surgiu a formulação indireta dos métodos de contorno, cuja base consiste em obter soluções físicas a partir de fontes fictícias aplicadas no contorno. Em 1967, surgiu a primeira formulação direta dos métodos de contorno, onde as variáveis são valores que possuem significados físicos. Esta formulação surgiu com o trabalho de Rizzo (1967), que apresentava o método das integrais de contorno como um método numérico. Surgiram vários trabalhos após o de Rizzo (1967), os quais serviram para divulgar o método das equações integrais de contorno, destacando-se os trabalhos de Cruse \& Rizzo (1968), Cruse (1968) apud Barbirato (1999), Cruse (1969), entre outros.

A partir dos trabalhos citados, Lachat (1975) apud Barbirato (1999) e principalmente Brebbia (1978), o qual apresentou uma formulação do método das equações integrais de contorno a partir da técnica de resíduos ponderados, este método passou a ser denominado de "Método dos Elementos de Contorno" (MEC). Desde então, o MEC vem se desenvolvendo, passando a ser estudado em muitos centros de pesquisa em todo o mundo, servindo para análises em mecânica dos

\footnotetext{
${ }^{1}$ Para maiores detalhes, ver notas biográficas em SIMMONS, G. F. (1987). Cálculo com geometria analítica. Tradução de Seiji Hariki. V.1. São Paulo, McGraw-Hill. No segundo volume desta obra é apresentada uma solução deste problema. 2 FREDHOLM, I. (1903). Sur une classe d'équations fonctionelles. Acta Math., v. 27, pp. 365-390.
} 
sólidos, mecânica dos fluidos, propagação de ondas, acústica, entre outras aplicações. Podendo ainda ser acoplado com outros métodos numéricos, como o Método dos Elementos Finitos.

A elasticidade tridimensional estática e dinâmica tem sido um campo bastante estudado, onde o primeiro trabalho nesta área deve-se a Cruse (1969). Surgiram vários trabalhos nesta área, destacando-se no Brasil os trabalhos de Nakaguma (1979), Curotto (1981) apud Barbirato (1999), Sá \& Telles (1986), Barbirato (1991), Coda (1993), Calderón (1996), Barbirato (1999), entre outros.

No presente trabalho estudar-se-á problemas com a elasticidade tridimensional estática através do Método dos Elementos de Contorno, mas utilizando uma nova abordagem. Ao invés de utilizar apenas a solução fundamental como função ponderadora, também se faz uso das soluções hiper-singulares, ou ainda uma combinação entre tais funções. As soluções hiper-singulares têm sido largamente utilizadas em aplicações na área de mecânica da fratura, mas com uma abordagem diferente da que se propõe neste trabalho. Alguns trabalhos mais recentes utilizaram as soluções hiper-singulares como funções ponderadoras na resolução do problema potencial bidimensional, dentre os quais se destacam: Rudolphi (1989), Ingber \& Rudolphi (1990) e Telles \& Prado (1993), entre outros. Rudolphi (1989) utiliza a derivada tangente ao elemento e a solução fundamental no mesmo nó, gerando um elemento com grau de liberdade igual a quatro, e, portanto uma função aproximadora cúbica. Os resultados apresentados por ele são bastante satisfatórios para este elemento, sugerindo que possa ser utilizado no problema elástico.

Ingber \& Rudolphi (1990) formulam uma técnica híbrida, utilizando-se a derivada normal em alguns pontos estratégicos para tentarem reduzir 0 condicionamento da matriz do sistema linear. Formulam os três tipos a seguir:

1. Derivada normal para pontos onde o fluxo é prescrito (condição de contorno de Dirichlet) e a solução fundamental clássica onde o potencial é prescrito (condição de contorno de Neumann);

2. Solução clássica para pontos onde o fluxo é prescrito e a derivada normal onde o potencial é prescrito;

3. Solução fundamental clássica em todos os nós. 
Além disso, apresentam exemplos numéricos e comparam o erro absoluto obtido em vários pontos do domínio com o número de condicionamento, que diz o quanto o sistema linear é consistente, quanto menor o número de condicionamento maior a consistência do sistema linear. Concluem que, para contorno suave, todas as três técnicas produzem valores aceitáveis, sendo que a segunda formulação é a que apresenta resultados mais precisos apesar de possuir um número de condicionamento elevado, mas concluem que para o problema potencial bidimensional o número de condicionamento não é tão elevado que possa tornar o sistema linear muito inconsistente, devido àsolução fundamental ser logarítmica.

Telles \& Prado (1993) utilizam-se da solução hiper-singular, a derivada normal, também para o problema potencial, e comparam com a solução fundamental clássica, mas não apresentam resultados conclusivos a respeito da utilização da solução hiper-singular, e sugerem que sejam feitos mais testes combinando as duas soluções.

Além da aplicação de soluções fundamentais hiper-singulares, pretende-se, neste trabalho, implementar técnicas de integração singulares e quase-singulares gerais e precisas, tornando possível a solução de problemas esbeltos e a comparação entre as diversas soluções fundamentais empregadas. Espera-se gerar formulação confiável do MEC possibilitando, no futuro, a elaboração de software específico para o ensino e desenvolvimento científico de problemas mais avançados. 


\section{CAPÍTULO 3}

\section{CONCEITOS DE TEORIA DE ELASTICIDADE TRIDIMENSIONAL}

\subsection{Introdução}

Será mostrada a notação matemática que deverá ser utilizada em muitas expressões ao longo do texto. Depois serão apresentadas as relações da elasticidade estática para sólidos tridimensionais, mostrando as principais equações e constantes necessárias àformulação do MEC.

\subsection{Notação indicial}

Várias expressões apresentadas neste texto são escritas utilizando-se a notação indicial. Dessa forma as expressões ficam mais elegantes e seu tratamento mais ágil na implementação do código computacional.

O sistema de coordenadas cartesianas, normalmente representado pelos eixos $x, y$ e $z$, será representado por $x_{1}, x_{2}$ e $x_{3}$, respectivamente; ou de maneira genérica por $x_{i}$.

Todas as variáveis relacionadas com o índice i referem-se à direção cartesiana i. Por exemplo, deslocamento $u_{i}$, força de superfície $p_{i}$, entre outras.

Quando aparecer um índice repetido em uma expressão, este se refere a um somatório. Por exemplo: 


$$
\begin{aligned}
& a_{j}=u_{1 j} \cdot p_{1}+u_{2 j} \cdot p_{2}+u_{3 j} \cdot p_{3}=\sum_{i=1}^{3} u_{i j} \cdot p_{i}=u_{i j} \cdot p_{i}, \quad \mathrm{i}=\mathrm{j}=1,2,3 \\
& a_{k k}=a_{11}+a_{22}+a_{33}, \quad \mathrm{k}=1,2,3
\end{aligned}
$$

O delta de Kronecker, determinado pelo símbolo $\delta_{\mathrm{ij}}$, utilizado neste trabalho, tem a seguinte definição:

$$
\delta_{i j}= \begin{cases}1, & \text { se } i=j \\ 0, & \text { se } i \neq j\end{cases}
$$

As derivadas parciais usualmente apresentadas em livros de cálculo serão representadas apenas por uma vírgula. Por exemplo:

$$
\begin{gathered}
\frac{\partial \xi_{i}}{\partial x_{k}}=\xi_{i},{ }_{k} \\
\frac{\partial u_{i j}}{\partial x_{k}}=u_{i j},_{k}
\end{gathered}
$$

Informações adicionais sobre a notação indicial aqui apresentada podem ser encontradas em Brebbia \& Dominguez (1992) ou em Brebbia et al. (1984).

\subsection{Equações de equilíbrio}

As forças atuantes em um corpo são admitidas como sendo de dois tipos: forças volumétricas e forças de superfície. As forças volumétricas são determinadas por unidade de volume, pois atuam sobre o volume do corpo. Já as forças de superfície são determinadas por unidade de área, pois estas atuam apenas sobre a superfície do corpo. 

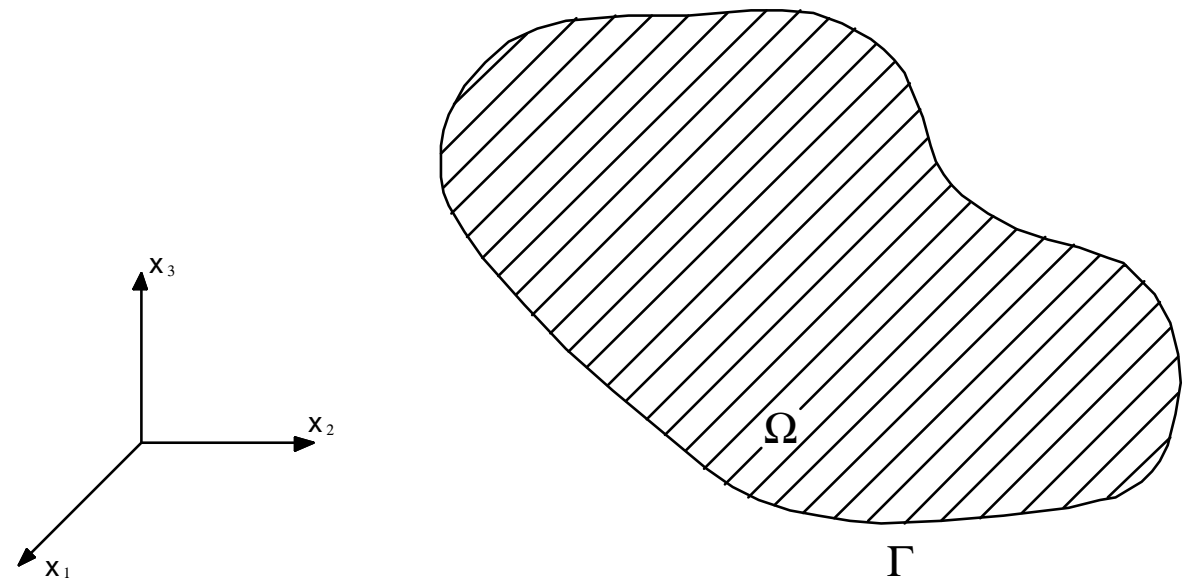

Figura 3.1 - Corpo tridimensional

Considerando-se um elemento infinitesimal, em forma de um paralelepípedo (figura 3.2), de um corpo tridimensional, isotrópico, elástico-linear e homogêneo, definido por um domínio $\Omega$ e contorno $\Gamma$ (figura 3.1), sobre o qual atuam forças volumétricas b e de superfície $p$, o equilíbrio de um ponto qualquer deste corpo é regido pela seguinte relação:

$$
\mathrm{o}_{i j,}+\mathrm{b}_{j}=0, \quad \mathrm{i}, \mathrm{j}=1,2,3
$$

Onde:

$$
\begin{aligned}
& \sigma_{i j} \text { : é o tensor de tensões } \\
& b_{j} \text { : é o vetor de forças volumétricas }
\end{aligned}
$$

Além disso, se não existir momentos volumétricos aplicados, a condição de equilíbrio do corpo nos garante a simetria existente no tensor de tensões:

$$
\sigma_{i j}=\sigma_{j i}
$$

As componentes das forças de superfície atuantes em um ponto qualquer do corpo podem ser expressas através das seis componentes do tensor de tensões, essa expressão é conhecida como fórmula de Cauchy':

\footnotetext{
${ }^{1}$ CAUCHY, A. L. (1828). Exercices de mathématique. Sur l'équilibre et le mouvement d'un systéme de points matériels par des forces d'attraction ou de répulsion mutuelle.
} 


$$
p_{i}=\sigma_{i j} \cdot n_{j}, \quad \mathrm{i}=\mathrm{j}=1,2,3
$$

Onde $n$ representa os co-senos diretores dos ângulos entre a normal $n$, do plano tangente que passe através do ponto em estudo (figura 3.2), e o eixo $x_{j}$.

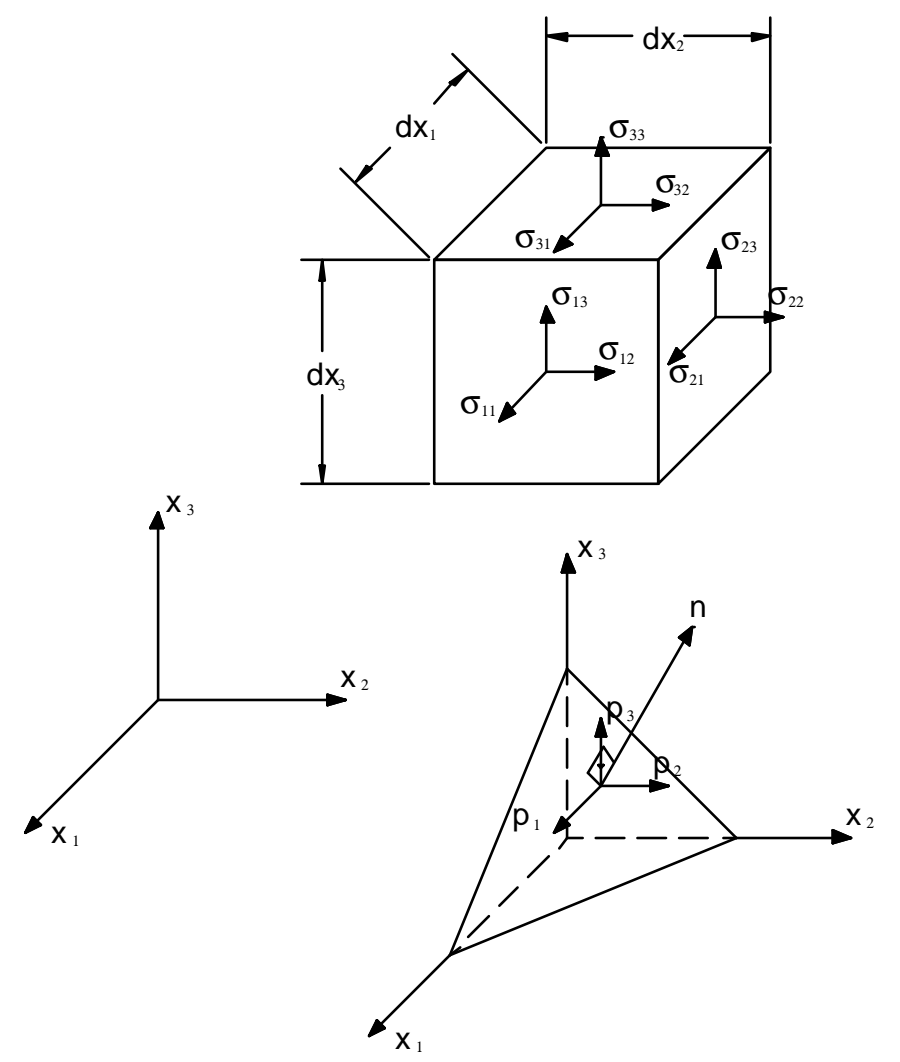

Figura 3.2 - Tensões internas e forças de superfície

\subsection{Relações entre deformação e deslocamento}

Para um corpo deformado em que apenas se considerem pequenas deformações, estas podem ser relacionadas com os deslocamentos através da relação:

$$
\varepsilon_{i j}=\frac{1}{2}\left(u_{i},{ }_{j}+u_{j},,_{i}\right), \quad \mathrm{i}=\mathrm{j}=1,2,3
$$




\subsection{Relações entre tensão e deformação}

As tensões e deformações de um corpo linearmente elástico, isotrópico e homogêneo são relacionadas através da lei de Hooke":

$$
\sigma_{i j}=\frac{2 \cdot G \cdot v}{1-2 \cdot v} \cdot \varepsilon_{k k} \cdot \delta_{i j}+2 \cdot G \cdot \varepsilon_{i j}, \quad i=\mathrm{j}=1,2,3
$$

Ou inversamente

$$
\varepsilon_{i j}=\frac{1}{2 \cdot G}\left(\sigma_{i j}-\frac{v}{1+\nu} \cdot \sigma_{k k} \cdot \delta_{i j}\right), \quad i=j=1,2,3
$$

Ou de outra forma:

$$
\dot{o}_{i j}=\ddot{e} \cdot \stackrel{\circ}{k}_{k k} \cdot \ddot{a}_{i j}+2 \cdot G \cdot \stackrel{\circ}{a j}_{i j}
$$

E que ainda pode ser escrita de forma mais compacta:

$$
\sigma_{i j}=C_{i j}^{k l} \cdot \varepsilon_{k l}
$$

Com:

$$
C_{i j}^{k l}=\frac{2 \cdot G \cdot v}{1-2 \cdot v} \cdot \delta_{i j} \cdot \delta_{k l}+G \cdot\left(\delta_{i k} \cdot \delta_{j l}+\delta_{i l} \cdot \delta_{j k}\right)
$$

Onde:

$v$ : coeficiente de Poisson ${ }^{2}$

E: módulo de elasticidade longitudinal

$\delta_{\mathrm{ij}}$ : delta de Kronecker

$\lambda$ e G: constantes de Lamé, sendo G também conhecido como módulo de elasticidade transversal.

As constantes de Lamé são escritas em função do coeficiente de Poisson e do módulo de elasticidade longitudinal:

\footnotetext{
${ }^{1}$ HOOKE, Robert. (1678). De potentia restitutiva, Londres

2 POISSON, S. D. (1829). Mémoire sur l'équilibre et le mouvement des corps élastiques. Paris, Mém. Acad. Vol. 8.
} 


$$
\begin{aligned}
& \ddot{e}=\frac{E \cdot i}{(1-2 \cdot i) \cdot(1+i)} \\
& G=\frac{E}{2 \cdot(1+v)}
\end{aligned}
$$

Substituindo-se a equação (3.9) na equação (3.10c), tem-se a expressão que relaciona diretamente as tensões com o campo de deslocamentos:

$$
\delta_{i j}=\ddot{e} \cdot u_{k, k} \cdot \ddot{a}_{i j}+G \cdot\left(u_{i, j}+u_{j, i}\right), \mathrm{i}=\mathrm{j}, \mathrm{k}=1,2,3
$$

\subsection{Equação de Navier-Cauchy}

A equação diferencial que governa os deslocamentos de um corpo sólido tridimensional é denominada de equação de Navier-Cauchy ${ }^{1}$, a qual é obtida através da substituição da equação (3.9) na equação (3.10a) e a resultante na (3.6), ou simplesmente a equação (3.14) na (3.6).

$$
u_{j, k k}+\frac{1}{1-2 \cdot v} u_{k},{ }_{k j}+\frac{b_{j}}{G}=0
$$

Ou ainda:

$$
G \cdot u_{j, k k}+(\lambda+G) \cdot u_{k, k j}+b_{j}=0
$$

\subsection{Condições de contorno}

Para se resolver um problema elástico é necessário que se conheçam as condições de contorno do corpo tridimensional.

\footnotetext{
${ }^{1}$ NAVIER, L. M. H. (1864). Resumé des leçons données àl'école des ponts et chaussés sur l'application de la mechanique à l'établissement des constructions et des machines, Paris, 3th. Ed.
} 
Considere o contorno $\Gamma$ de um corpo sólido, composto por $\Gamma_{1}$ e $\Gamma_{2}$, onde $\Gamma=$ $\Gamma_{1}+\Gamma_{2}$, de acordo com a figura 3.3.

Definem-se então os valores prescritos de contorno que serão utilizados na análise do problema (deslocamentos e forças de superfície):

$$
\begin{aligned}
& u_{i}(Q)=\bar{u}_{i}(Q), Q \in \Gamma_{1} \text { (condições de contorno essenciais) } \\
& p_{i}(S)=\bar{p}_{i}(S), \quad S \in \Gamma_{2} \text { (condições de contorno naturais) }
\end{aligned}
$$

Onde o traço sobre os valores indica valores prescritos. Lembrando que as condições de contorno não necessariamente são aplicadas em partes separadas do contorno.

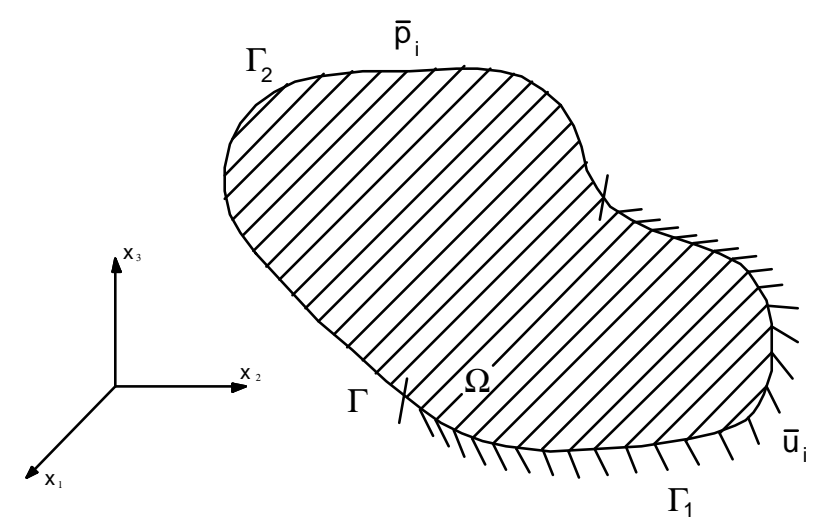

Figura 3.3 - Valores prescritos de contorno

\subsection{Delta de Dirac}

O conceito da distribuição Delta de Dirac é muito importante para a formulação do método dos elementos de contorno. A distribuição Delta de Dirac é uma função geral, a qual pode ser definida como o limite de uma função normal, a qual é zero para todos os pontos do domínio, exceto para o ponto em que o argumento da função é zero, neste ponto o limite tende para um valor infinito. Como definido na equação (3.19a-b)

$$
\left\{\begin{array}{l}
\delta(s, q)=0, \text { se } q \neq s \\
\delta(s, q)=\infty, \text { se } q=s
\end{array}\right.
$$


Além disso, a distribuição Delta de Dirac possui uma propriedade muito utilizada na engenharia:

$\int_{\Omega} \delta(s, q) \cdot 1 \cdot d \Omega=1$

E para uma função contínua qualquer, tem-se:

$\int_{\Omega} f(q) \cdot \delta(s, q) \cdot d \Omega=f(s)$

Maiores informações a respeito da função delta de Dirac podem ser obtidas em Brebbia et al. (1984). 


\section{CAPÍTULO 4}

\section{SOLUÇÃO FUNDAMENTAL}

\subsection{Introdução}

Para desenvolver uma formulação do método dos elementos de contorno é necessário um conhecimento prévio da chamada solução fundamental, que é escolhida de acordo com o problema a ser solucionado.

A solução fundamental que será utilizada neste trabalho foi desenvolvida por William Thompson ${ }^{1}$ (Lorde Kelvin), e é conhecida por solução fundamental de Kelvin.

A solução fundamental de Kelvin é definida como a resposta de um corpo elástico e de domínio infinito, submetido àação de uma força estática e unitária.

\subsection{Solução fundamental de Kelvin}

A solução fundamental de Kelvin, por ser a mais abrangente e mais simples de ser implementada, tornou-se a mais utilizada e difundida pela área técnica. Esta solução foi desenvolvida por Kelvin, de acordo com Love (1944), e é determinada

\footnotetext{
${ }^{1}$ LOVE, A. E. H. (1944). A treatise on the mathematical theory of elasticity. $4^{\text {th }}$. Ed. New York, Dover Publications.
} 
considerando-se um domínio $\Omega$, como um sólido elástico, isotrópico, homogêneo e infinito, como mostrado na figura 4.1.

As expressões encontradas para a solução do problema fundamental de Kelvin em termos de deslocamentos e forças de superfície, para o estado tridimensional, são as seguintes:

$$
u_{i j}^{*}(s, q)=\frac{1}{16 \pi G(1-v) r}\left[(3-4 v) \cdot \delta_{i j}+r,,_{i} \cdot r,{ }_{j}\right]
$$

Onde o primeiro índice refere-se à direção da carga unitária aplicada e o segundo a direção do efeito provocado, como mostrado na figura 4.2.

Pela definição apresentada na equação (3.9), tem-se que:

$$
\varepsilon_{i j k}^{*}(s, q)=\frac{-1}{16 \pi G(1-v) r^{2}}\left[(1-2 v) \cdot\left(r,{ }_{j} \cdot \delta_{i k}+r,{ }_{i} \cdot \delta_{j k}\right)-r,,_{k} \cdot \delta_{i j}+3 \cdot r,,_{i} \cdot r,{ }_{j} \cdot r,{ }_{k}\right]
$$

Aplicando-se a lei de Hooke, tem-se:

$$
\sigma_{i j k}^{*}(s, q)=\frac{-1}{8 \pi(1-v) r^{2}}\left[(1-2 v) \cdot\left(r,,_{j} \cdot \delta_{i k}+r,_{i} \cdot \delta_{j k}-r,{ }_{, k} \cdot \delta_{i j}\right)+3 \cdot r,_{i} \cdot r,{ }_{j} \cdot r,_{k}\right]
$$

Enfim, aplicando-se a fórmula de Cauchy (equação 4.8):

$$
P_{i j}^{*}(s, q)=\frac{-1}{8 \pi(1-v) r^{2}}\left\{\left[(1-2 v) \cdot \delta_{i j}+3 \cdot r,{ }_{i} \cdot r,{ }_{j}\right] \frac{\partial r}{\partial n}-(1-2 v) \cdot\left(r,{ }_{i} \cdot n_{j}-r,{ }_{j} \cdot n_{i}\right\}\right.
$$




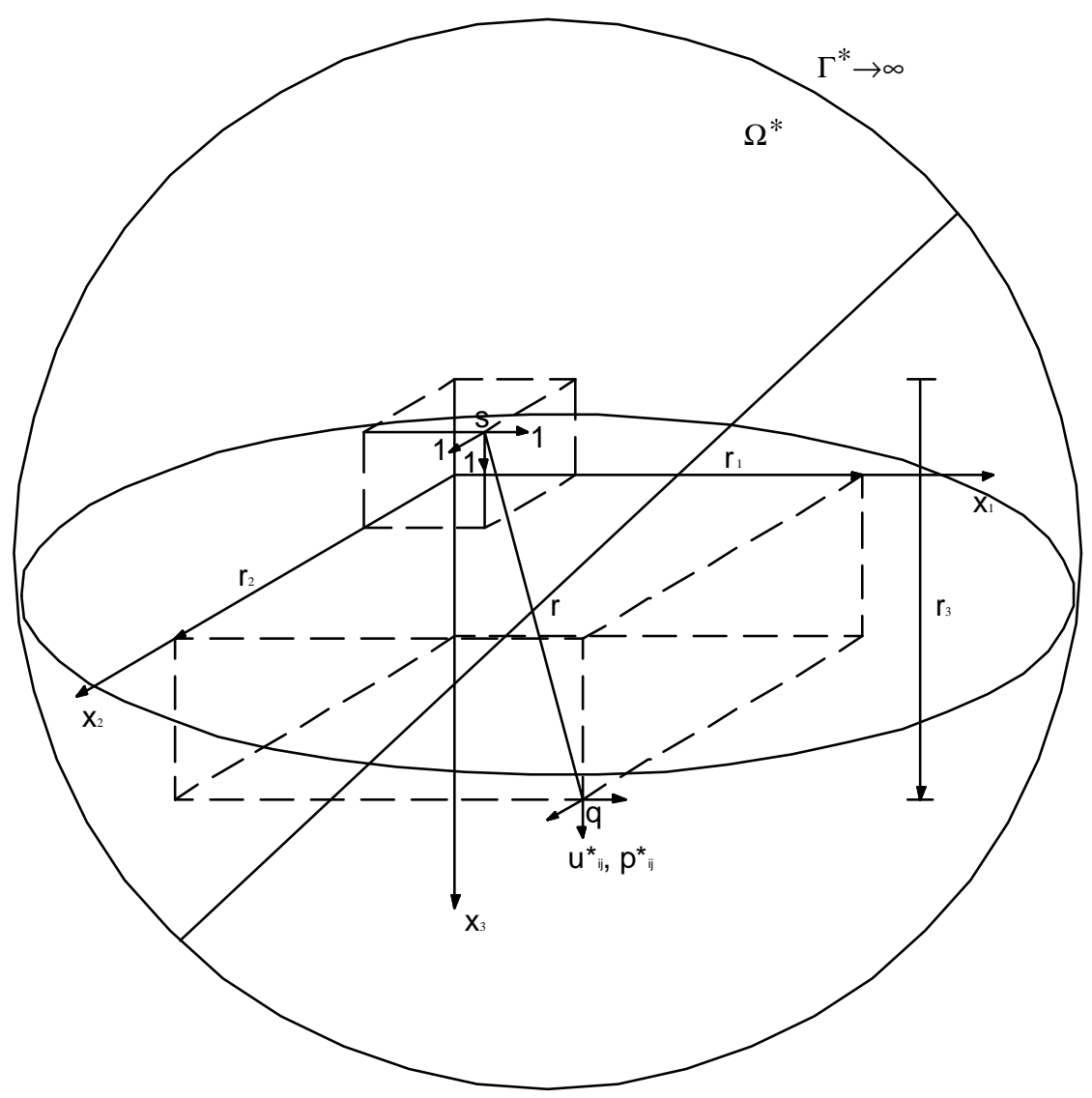

Figura 4.1 - Problema fundamental de Kelvin

Onde:

$\Omega^{*}$ : domínio que se estende ao infinito

$\Gamma^{*}$ : contorno que também se estende ao infinito

$\mathrm{x}_{\mathrm{i}}$ : corresponde aos eixos cartesianos

r: distância entre os pontos s e q

$r_{i}$ : componentes cartesianas de $r$

s: ponto fonte com as forças unitárias nas três direções

q: ponto onde serão avaliadas as respostas æ̀ forças unitárias

$\mathrm{u}_{\mathrm{ij}}^{*}$ : deslocamentos fundamentais

$\mathrm{p}_{\mathrm{ij}}^{*}$ : forças de superfície fundamentais 


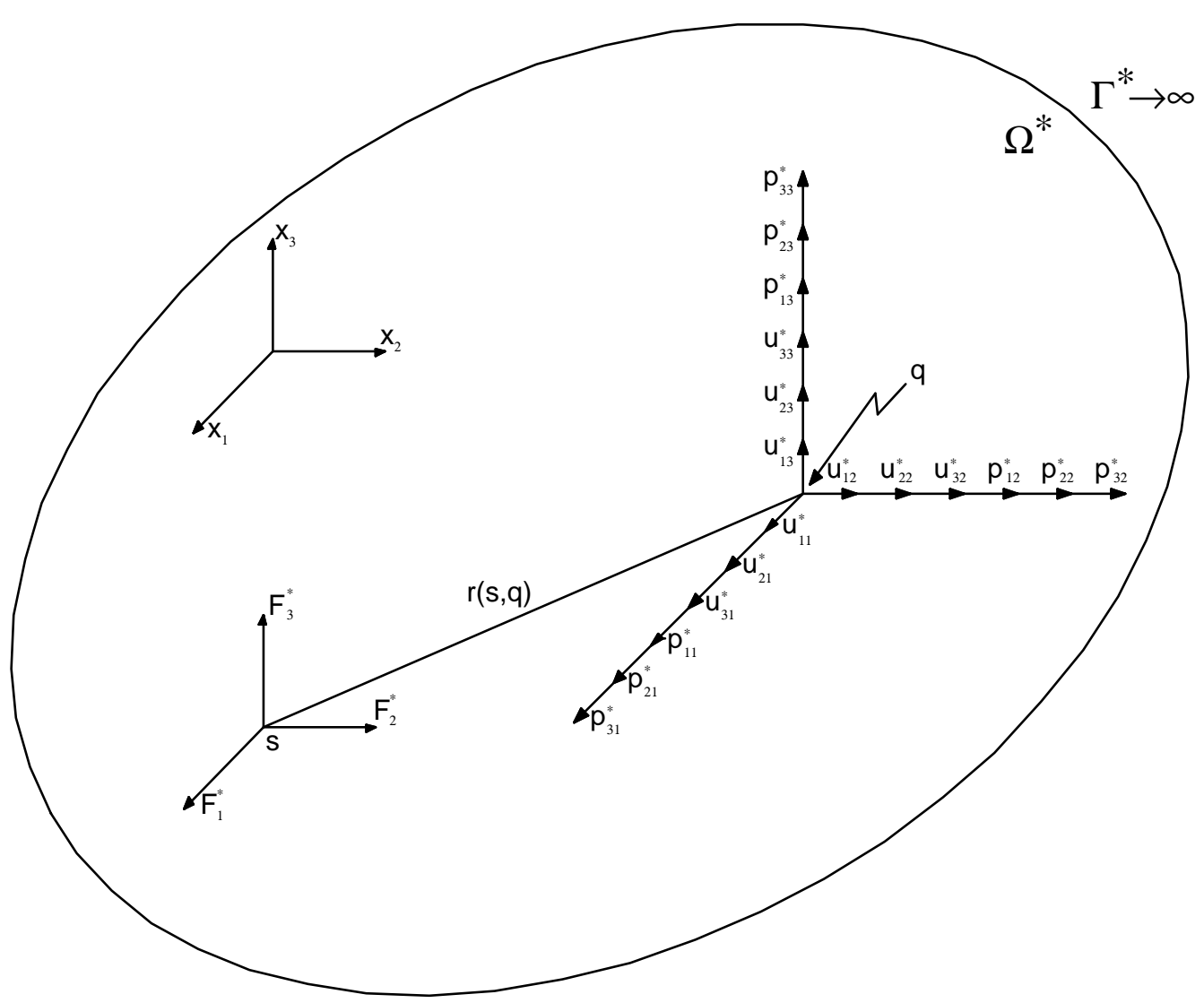

Figura 4.2 - Componentes dos tensores de deslocamentos e forças de superfície

\subsection{Solução hiper-singular}

Uma solução hiper-singular é um ponderador com ordem de singularidade maior do que a solução fundamental.

A solução hiper-singular utilizada neste trabalho é derivada da solução fundamental de Kelvin. É obtida derivando-se a solução fundamental de Kelvin segundo uma direção qualquer.

As expressões obtidas para as derivadas de deslocamentos e forças de superfície são as seguintes:

$$
\frac{\partial u_{i j}^{*}(s, q)}{\partial x_{k}(s)}=u_{i j, k}^{*}(s, q)=\frac{-1}{16 \pi G(1-v) r^{2}}\left[-(3-4 v) \cdot r,_{k} \cdot \delta_{i j}+r,{ }_{i} \cdot \delta_{j k}+r,{ }_{j} \cdot \delta_{i k}-3 \cdot r,,_{i} \cdot r,{ }_{j} \cdot r,,_{k}\right]
$$




$$
\begin{gathered}
\frac{\partial P_{i j}^{*}(s, q)}{\partial x_{k}(s)}=P_{i j}^{*}{ }_{k}(s, q)=\frac{-1}{8 \pi(1-v) r^{3}}\left\{3 \cdot \frac{\partial r}{\partial n} \cdot\left[(1-2 v) \cdot r,{ }_{, k} \delta_{i j}-r,{ }_{i} \delta_{j k}-r,{ }_{j} \cdot \delta_{i k}+5 \cdot r,,_{i} \cdot r,{ }_{j} \cdot r_{, k}\right]\right. \\
-3 \cdot r_{, i} \cdot r,{ }_{j} \cdot n_{k}+(1-2 v) \cdot\left(-3 \cdot r,{ }_{i} \cdot r_{,} \cdot n_{j}+3 \cdot r,,_{j} \cdot r,{ }_{k} \cdot n_{i}\right. \\
\left.-n_{i} \cdot \delta_{j k}+n_{j} \cdot \delta_{i k}-n_{k} \cdot \delta_{i j}\right\}
\end{gathered}
$$

A partir das expressões (4.5) e (4.6) pode-se facilmente determinar as derivadas destas na direção normal aos elementos de contorno, como mostrado a seguir:

$$
\begin{aligned}
& \frac{\partial u_{i j}^{*}}{\partial n}=\frac{\partial u_{i j}^{*}}{\partial x_{l}} \cdot n_{l} \\
& \frac{\partial P_{i j}^{*}}{\partial n}=\frac{\partial P_{i j}^{*}}{\partial x_{l}} \cdot n_{l}
\end{aligned}
$$

Com I = 1, 2 e 3. 


\section{CAPÍTULO 5}

\section{EQUAÇÃO INTEGRAL DE CONTORNO}

\subsection{Introdução}

Para se desenvolver uma formulação para o método dos elementos de contorno é necessário que se tenha conhecimento das equações integrais de contorno, as quais relacionam deslocamentos de um ponto qualquer do domínio com deslocamentos e esforços no contorno de um corpo tridimensional através de integrais envolvendo as soluções fundamentais. Tais equações podem ser obtidas através da técnica dos resíduos ponderados, utilizando a solução fundamental como função ponderadora, ou através do teorema da reciprocidade de Betti ${ }^{1}$. Neste trabalho utilizar-se-á a técnica dos resíduos ponderados, pois através desta o método dos elementos de contorno consegue ser associado a outros métodos numéricos, como, por exemplo, o método das diferenças finitas e o método dos elementos finitos.

${ }^{1}$ BETTI, E. (1872). Teoria dell elasticita. II Nuovo Cimento. (Ser. 3), v6-10. 


\subsection{Equação integral para pontos do domínio}

Seja considerado um espaço infinito e que contenha um sólido tridimensional de domínio $\Omega$ e contorno $\Gamma$ dividido em $\Gamma_{1}$ (deslocamentos prescritos) e $\Gamma_{2}$ (forças de superfície prescritas).

$$
\begin{aligned}
& u_{i}=\bar{u}_{i} \quad \text { em } \Gamma_{1} \text { (condições de contorno essenciais) } \\
& p_{i}=\bar{p}_{i} \quad \text { em } \Gamma_{2} \text { (condições de contorno naturais) }
\end{aligned}
$$

Onde o traço em cima dos valores $u_{i}$ e $p_{i}$ indica valores prescritos de deslocamentos e forças de superfície respectivamente.

Através da técnica dos resíduos ponderados e utilizando-se uma solução fundamental qualquer como função ponderadora para aproximar numericamente a equação de equilíbrio da estática (equação 3.6), obtém-se:

$$
\left(\sigma_{i j, i}+b_{j}\right) \cdot u_{j}^{*}=0
$$

Efetuando-se uma integral em todo o domínio do corpo estudado, a igualdade (5.3) não se altera, portanto:

$$
\int_{\Omega} \sigma_{i j, i} \cdot u_{j}^{*} \cdot d \Omega+\int_{\Omega} b_{j} \cdot u_{j}^{*} \cdot d \Omega=0
$$

A primeira integral da expressão (5.4) pode ser transformada para uma integral no contorno através de uma integração por partes:

$$
\int_{\Omega} \sigma_{i j},{ }_{i} \cdot u_{j}^{*} \cdot d \Omega=\int_{\Gamma} \sigma_{i j} \cdot n_{i} \cdot u_{j}^{*} \cdot d \Gamma-\int_{\Omega} \sigma_{i j} \cdot u_{j}^{*},{ }_{i} \cdot d \Omega
$$

Aplicando-se a fórmula de Cauchy (3.8) na integral sobre o contorno $\Gamma$ :

$$
\int_{\Omega} \sigma_{i j},{ }_{i} \cdot u_{j}^{*} \cdot d \Omega=\int_{\Gamma} p_{j} \cdot u_{j}^{*} \cdot d \Gamma-\int_{\Omega} \sigma_{i j} \cdot u_{j}^{*}, \cdot d \Omega
$$

Levando-se em consideração a simetria do tensor de tensões e a definição apresentada em (3.9): 


$$
\sigma_{i j} \cdot u_{j}^{*},{ }_{i}=\sigma_{i j} \cdot \varepsilon_{j i}^{*}=\sigma_{i j} \cdot \varepsilon_{i j}^{*}
$$

Sendo:

$\varepsilon_{i j}^{*}$ : campo de deformação do problema fundamental

Substituindo a relação obtida em (5.7) na integral sobre o domínio no lado direito da igualdade (5.6) e o resultado em (5.4), tem-se:

$$
\int_{\Gamma} p_{j} \cdot u_{j}^{*} \cdot d \Gamma-\int_{\Omega} \sigma_{i j} \cdot \varepsilon_{i j}^{*} \cdot d \Omega+\int_{\Omega} b_{j} \cdot u_{j}^{*} \cdot d \Omega=0
$$

Para se chegar a equação integral final, ainda é necessário se trabalhar a integral sobre o domínio, que contém o tensor de tensões, da expressão (5.8). Pela Lei de Hooke, tem-se:

$$
\sigma_{i j} \cdot \varepsilon_{i j}^{*}=C_{i j}^{k l} \cdot \varepsilon_{k l} \cdot \varepsilon_{i j}^{*}=\varepsilon_{k l} \cdot C_{i j}^{k l} \cdot \varepsilon_{i j}^{*}=\varepsilon_{k l} \cdot C_{k l}^{i j} \cdot \varepsilon_{i j}^{*}=\varepsilon_{k l} \cdot \sigma_{k l}^{*}
$$

A penúltima passagem na expressão (5.9) é devido à simetria do tensor constitutivo elástico. E de forma análoga, mas inversa, àpropriedade (5.7), tem-se:

$$
\varepsilon_{i j} \cdot \sigma_{i j}^{*}=u_{j}, \sigma_{i}{ }_{i j}
$$

Portanto a integral sobre o domínio na expressão (5.8), fica:

$$
\int_{\Omega} \sigma_{i j} \cdot \varepsilon_{i j}^{*} \cdot d \Omega=\int_{\Omega} u_{j}, \sigma_{i j}^{*} \cdot d \Omega
$$

Agora é preciso transformar esta integral para uma integral sobre o contorno através de uma integração por partes:

$$
\int_{\Omega} u_{j},{ }_{i} \cdot \sigma_{i j}^{*} \cdot d \Omega=\int_{\Gamma} u_{j} \cdot n_{i} \cdot \sigma_{i j}^{*} \cdot d \Gamma-\int_{\Omega} u_{j} \cdot \sigma_{i j}^{*},_{i} \cdot d \Omega
$$

Aplicando-se a fórmula de Cauchy na integral sobre o contorno da expressão (5.12), e levando-se esse resultado à expressão (5.8), de acordo com a igualdade (5.11), obtém-se:

$$
\int_{\Gamma} p_{j} \cdot u_{j}^{*} \cdot d \Gamma-\int_{\Gamma} u_{j} \cdot p_{j}^{*} \cdot d \Gamma+\int_{\Omega} u_{j} \cdot \sigma_{i j}^{*},{ }_{i} \cdot d \Omega+\int_{\Omega} b_{j} \cdot u_{j}^{*} \cdot d \Omega=0
$$


Lembrando ainda que, sendo o campo de deslocamento fundamental, elástico e estático, respeitando assim a equação diferencial, tem-se:

$$
\sigma_{i j}^{*},{ }_{i}+b_{j}^{*}=0
$$

E, portanto a expressão (5.13) fica escrita em sua forma final:

$$
\int_{\Gamma} p_{j} \cdot u_{j}^{*} \cdot d \Gamma+\int_{\Omega} b_{j} \cdot u_{j}^{*} \cdot d \Omega=\int_{\Gamma} u_{j} \cdot p_{j}^{*} \cdot d \Gamma+\int_{\Omega} b_{j}^{*} \cdot u_{j} \cdot d \Omega
$$

Para transformar a expressão (5.15) numa forma integral sobre a qual se possa realizar uma análise numérica, é preciso uma breve explicação do problema fundamental, como é descrito pela equação (5.14). Mas para um melhor entendimento torna-se necessário dividir o sistema de equações (5.14) nas três equações que representam o problema:

$$
\begin{aligned}
& \sigma_{i 1, i}+b_{1}^{*}=0 \\
& \sigma_{i 2, i}+b_{2}^{*}=0 \\
& \sigma_{i 3, i}+b_{3}^{*}=0
\end{aligned}
$$

O problema fundamental é constituído por 3 casos de carregamento. No primeiro aplica-se apenas uma força concentrada e unitária na direção 1, portanto:

$$
\begin{aligned}
& \sigma_{1 i 1, i}+\delta^{*}(s, q)=0 \\
& \sigma_{1 i 2, i}+0=0 \\
& \sigma_{1 i 3, i}+0=0
\end{aligned}
$$

Onde:

$\delta^{*}(s, q)$ : distribuição Delta de Dirac.

$\mathrm{Na}$ equação (5.17), o índice adicional "1" representa o primeiro problema fundamental e, portanto, a direção do carregamento concentrado.

No segundo problema fundamental, a força concentrada e unitária atua na direção 2, o que resulta:

$$
\begin{aligned}
& \sigma_{2 i 1, i}+0=0 \\
& \sigma_{2 i, i}+\delta^{*}(s, q)=0 \\
& \sigma_{2 i 3, i}+0=0
\end{aligned}
$$


E de forma análoga, tem-se para o terceiro problema fundamental:

$$
\begin{aligned}
& \sigma_{3 i 1, i}+0=0 \\
& \sigma_{3 i 2, i}+0=0 \\
& \sigma_{3 i 3, i}+\delta^{*}(s, q)=0
\end{aligned}
$$

E de maneira geral, tem-se:

$$
\sigma_{k i j,}^{*}+b_{k j}^{*}=0
$$

Onde:

$$
b_{k j}^{*}=\delta_{k j} \cdot \delta(s, q)
$$

Sendo $\delta_{\mathrm{kj}}$ O delta de Kronecker. O conjunto de soluções dos problemas " $\mathrm{k}$ " denomina-se solução fundamental do problema elastostático $\left(u_{k j}^{*}\right)$.

Substituindo-se os estados $u_{k j}^{*}$ e de seu carregamento correspondente $b_{k j}^{*}$ na expressão (5.15) e trocando-se devidamente o índice "k" por "i", tem-se a equação integral de contorno para deslocamento.

$$
u_{i}(s)=\int_{\Gamma} p_{j}(Q) \cdot u_{i j}^{*}(s, Q) \cdot d \Gamma-\int_{\Gamma} u_{j}(Q) \cdot p_{i j}^{*}(s, Q) \cdot d \Gamma+\int_{\Omega} b_{j}(q) \cdot u_{i j}^{*}(s, q) \cdot d \Omega
$$

Esta equação é chamada de Identidade Somigliana ${ }^{1}$ que determina valores de deslocamentos para pontos internos através de deslocamentos e forças de superfície do contorno, $\mathrm{u}_{\mathrm{j}}$ e $\mathrm{p}_{\mathrm{j}}$ respectivamente.

Com a representação integral para deslocamentos em pontos internos, poderse-á determinar a representação integral para tensões nos pontos internos. Basta para isso aplicar a relação cinemática (3.9) sobre a equação (5.22), lembrando que a diferenciação será feita com relação à posição do ponto fonte $\mathrm{s}$, e após isso, utilizar a lei de Hooke (3.10). Obtendo-se assim a seguinte expressão:

$$
\sigma_{i j}(s)=\int_{\Gamma} D_{k i j}^{*}(s, Q) \cdot p_{k}(Q) \cdot d \Gamma-\int_{\Gamma} S_{k i j}^{*}(s, Q) \cdot u_{k}(Q) \cdot d \Gamma+\int_{\Omega} D_{k i j}^{*}(s, q) \cdot b_{k}(q) \cdot d \Omega
$$

\footnotetext{
${ }^{1}$ SOMIGLIANA, C. (1886). Sopra l'equilibrio di um corpo elástico isótropo. /l Nuovo Cimento. (Ser. 3), v. 17-20.
} 
Onde:

$D^{*}{ }_{\text {kij }}$ e $S_{\text {kij }}^{*}$ são tensores derivados dos tensores de deslocamentos e forças de superfície do problema fundamental, e suas componentes para a solução fundamental de Kelvin são:

$$
\begin{aligned}
& D_{k i j}^{*}(s, Q)=\frac{1}{8 \pi(1-v) r^{2}}\left\{(1-2 v) \cdot\left[\delta_{k i} \cdot r,{ }_{j}+\delta_{k j} \cdot r,{ }_{i}-\delta_{i j} \cdot r,{ }_{k}\right]+3 \cdot r,{ }_{i} \cdot r,{ }_{j} \cdot r,{ }_{k}\right\} \\
& S_{k i j}^{*}(s, Q)=\frac{G}{4 \pi(1-v) r^{3}} \cdot\left\{3 \cdot \frac{\partial r}{\partial n}\left[(1-2 v) \delta_{i j} \cdot r,{ }_{k}+v\left(\delta_{k i} \cdot r,{ }_{j}+\delta_{k j} \cdot r,_{i}\right)-5 \cdot r,,_{i} \cdot r,{ }_{j} \cdot r,{ }_{k}\right]\right. \\
& +3 \cdot v \cdot\left(n_{i} \cdot r,{ }_{j} \cdot r,{ }_{k}+n_{j} \cdot r,{ }_{i} \cdot r,{ }_{k}\right)+(1-2 v) \cdot\left(3 \cdot n_{k} \cdot r,{ }_{i} \cdot r,{ }_{j}\right. \\
& \left.\left.+n_{j} \delta_{i k}+n_{i} \delta_{j k}\right)-(1-4 v) n_{k} \delta_{i j}\right\}
\end{aligned}
$$

\subsection{Equação integral para pontos do contorno}

A Identidade Somigliana é válida apenas para pontos internos; como para determinar os valores de deslocamentos de pontos do domínio é necessário que se conheçam os valores de deslocamentos e forças de superfície de todos os pontos do contorno, então é preciso determinar os valores incógnitos de deslocamentos e forças de superfície de todos os pontos do contorno. Para isso é necessário escrever a equação integral de contorno para pontos do contorno, utilizando-se um artifício que consiste em transformar, inicialmente, o ponto de contorno em um ponto de domínio, sobre o qual pode-se aplicar a Identidade Somigliana. Esta técnica consiste em acrescentar o contorno de uma superfície esférica de domínio $\Omega_{\varepsilon}$, com centro no ponto fonte de contorno e de raio $\varepsilon$, conforme mostrado na figura 5.1. Dessa forma, o ponto do contorno transforma-se, transitoriamente, em um ponto do domínio, estabelecendo-se para ele a Identidade Somigliana com o domínio e contorno acrescidos da parte esférica. Feito isso, determina-se o limite das parcelas acrescidas anteriormente para $\varepsilon \rightarrow 0$, resultando assim a Identidade Somigliana para pontos do contorno. 


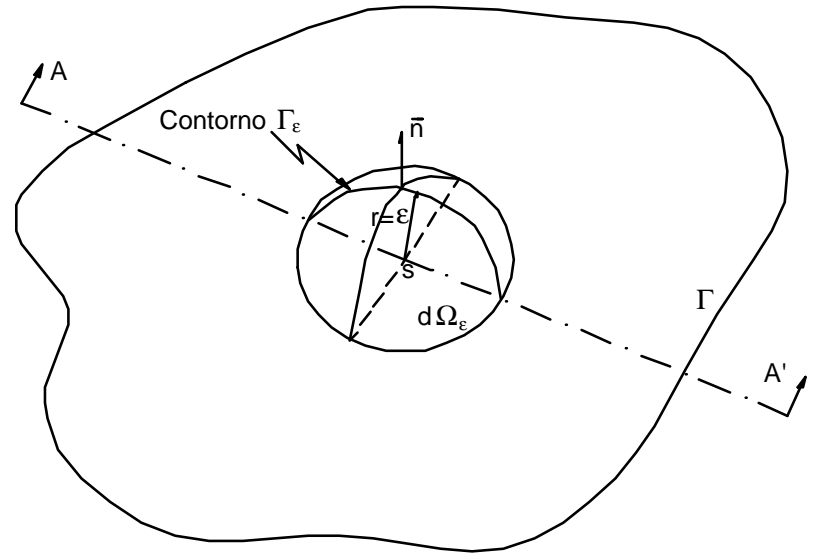

(a)

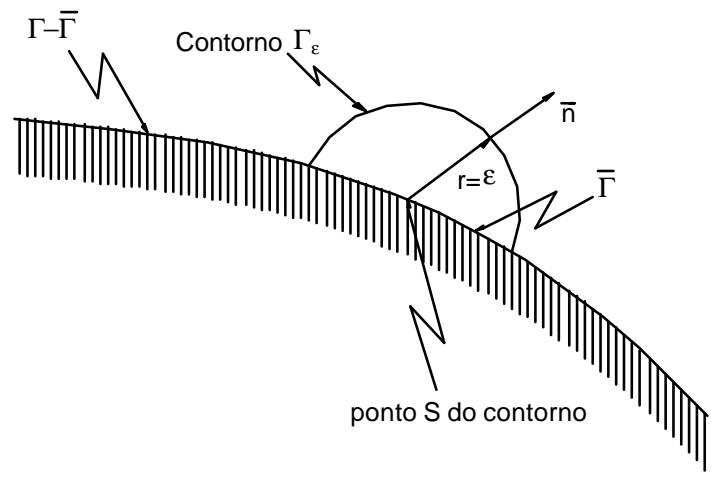

(b)

Figura 5.1 - (a) Contorno expandido no ponto S; (b) Corte AA'

Com o acréscimo do contorno, a Identidade Somigliana fica da seguinte forma:

$$
u_{i}(S)=\int_{\Gamma-\Gamma+\Gamma_{\varepsilon}} p_{j}(Q) \cdot u_{i j}^{*}(S, Q) \cdot d \Gamma-\int_{\Gamma-\bar{\Gamma}+\Gamma_{\varepsilon}} u_{j}(Q) \cdot p_{i j}^{*}(S, Q) \cdot d \Gamma+\int_{\Omega+\Omega_{\varepsilon}} b_{j}(q) \cdot u_{i j}^{*}(S, q) \cdot d \Omega \quad \text { (5.26) }
$$

Onde:

$\Gamma_{\varepsilon}:$ contorno da superfície esférica acrescida

$\bar{\Gamma}$ : parte do contorno que foi expandida

$\Omega_{\varepsilon}$ : domínio da parte esférica acrescida 
Desmembrando-se as partes das integrais que contêm os acréscimos de contorno e domínio, $\Gamma_{\varepsilon}$ e $\Omega_{\varepsilon}$, respectivamente, tem-se:

$$
\begin{aligned}
u_{i}(S)= & \int_{\Gamma-\bar{\Gamma}} p_{j}(Q) \cdot u_{i j}^{*}(S, Q) \cdot d \Gamma+\int_{\Gamma_{\varepsilon}} p_{j}(Q) \cdot u_{i j}^{*}(S, Q) \cdot d \Gamma-\int_{\Gamma-\bar{\Gamma}} u_{j}(Q) \cdot p_{i j}^{*}(S, Q) \cdot d \Gamma \\
& -\int_{\Gamma_{\varepsilon}} u_{j}(Q) \cdot p_{i j}^{*}(S, Q) \cdot d \Gamma+\int_{\Omega+\Omega_{\varepsilon}} b_{j}(q) \cdot u_{i j}^{*}(S, q) \cdot d \Omega
\end{aligned}
$$

Para que o ponto $S$ pertença ao contorno $\Gamma$ novamente é necessário fazer 0 limite de $\varepsilon \rightarrow 0$, assim $\Omega_{\varepsilon}, \Gamma_{\varepsilon}$ e $\bar{\Gamma}$ tenderão a zero também.

É preciso agora analisar as seguintes integrais quando se faz o limite $\varepsilon \rightarrow 0$ :

$$
\begin{aligned}
u_{i}(S)= & \lim _{\varepsilon \rightarrow 0} \int_{\Gamma-\bar{\Gamma}} p_{j}(Q) \cdot u_{i j}^{*}(S, Q) \cdot d \Gamma+\lim _{\varepsilon \rightarrow 0} \int_{\Gamma_{\varepsilon}} p_{j}(Q) \cdot u_{i j}^{*}(S, Q) \cdot d \Gamma \\
& -\lim _{\varepsilon \rightarrow 0} \int_{\Gamma-\bar{\Gamma}} u_{j}(Q) \cdot p_{i j}^{*}(S, Q) \cdot d \Gamma-\lim _{\varepsilon \rightarrow 0} \int_{\Gamma_{\varepsilon}} u_{j}(Q) \cdot p_{i j}^{*}(S, Q) \cdot d \Gamma \\
& +\lim _{\varepsilon \rightarrow 0} \int_{\Omega+\Omega_{\varepsilon}} b_{j}(q) \cdot u_{i j}^{*}(S, q) \cdot d \Omega
\end{aligned}
$$

A solução fundamental de Kelvin em termos de deslocamentos possui singularidade $1 / r$, como o contorno acrescentado é esférico, gerará um termo $r^{2}$ (a área de uma esfera é $4 \pi r^{2}$ ), portanto as integrais que contém deslocamentos fundamentais como núcleo podem ser avaliadas diretamente.

Analisando a primeira integral do lado direito da igualdade (5.28), tem-se:

$$
\lim _{\varepsilon \rightarrow 0} \int_{\Gamma-\bar{\Gamma}} p_{j}(Q) \cdot u_{i j}^{*}(S, Q) \cdot d \Gamma=\int_{\Gamma} p_{j}(Q) \cdot u_{i j}^{*}(S, Q) \cdot d \Gamma
$$

A segunda integral do lado direito da igualdade (5.28):

$$
\lim _{\varepsilon \rightarrow 0} \int_{\Gamma_{\varepsilon}} p_{j}(Q) \cdot u_{i j}^{*}(S, Q) \cdot d \Gamma=0
$$

O mesmo caso acontece para a quinta integral do lado direito da igualdade (5.28), pois quando $\varepsilon \rightarrow 0, \Omega_{\varepsilon}$ também tende a zero e como a integral não possui singularidade, pode ser avaliada diretamente:

$$
\lim _{\varepsilon \rightarrow 0} \int_{\Omega+\Omega_{\varepsilon}} b_{j}(q) \cdot u_{i j}^{*}(S, q) \cdot d \Omega=\int_{\Omega} b_{j}(q) \cdot u_{i j}^{*}(S, q) \cdot d \Omega
$$


A solução fundamental de Kelvin em termos de forças de superfície possui singularidade $1 / r^{2}$, e como o contorno gerará um termo $r^{2}$, as integrais contendo forças de superfície fundamentais não podem ser avaliadas diretamente, ou seja, terão que ser avaliadas em termos de valor principal de Cauchy.

Analisando a terceira integral, tem-se que:

$$
\lim _{\varepsilon \rightarrow 0} \int_{\Gamma-\bar{\Gamma}} u_{j}(Q) \cdot p_{i j}^{*}(S, Q) \cdot d \Gamma={\underset{\Gamma}{\Gamma}}_{j} u_{j}(Q) \cdot p_{i j}^{*}(S, Q) \cdot d \Gamma
$$

Onde $f$ indica que a integral está sendo avaliada em termos de valor principal de Cauchy.

Finalmente analisando a quarta integral:

$$
\begin{aligned}
\lim _{\varepsilon \rightarrow 0} \int_{\Gamma_{\varepsilon}} u_{j}(Q) \cdot p_{i j}^{*}(S, Q) \cdot d \Gamma= & \lim _{\varepsilon \rightarrow 0} \int_{\Gamma_{\varepsilon}}\left[u_{j}(Q)-u_{j}(S)\right] \cdot p_{i j}^{*}(S, Q) \cdot d \Gamma \\
& +u_{j}(S) \cdot \lim _{\varepsilon \rightarrow 0} \int_{\Gamma_{\varepsilon}} p_{i j}^{*}(S, Q) \cdot d \Gamma
\end{aligned}
$$

O primeiro termo do lado direito da igualdade (5.33) é nulo, pois o campo de deslocamentos obedece àcondição de Hölder:

$$
\left[u_{j}(Q)-u_{j}(S)\right] \leq A \cdot r(S, Q)^{\alpha}
$$

Onde:

$u_{j}(Q)$ e $u_{j}(S)$ : são os deslocamentos nos pontos $Q$ e $S$, respectivamente $r(S, Q)$ : distância entre os pontos $S$ e $Q$

A e $\alpha$ : constantes positivas, sendo $0 \leq \alpha \leq 1$

O segundo termo do lado direito da igualdade (5.33) irá gerar um termo livre, e caso o contorno seja suave, ou seja, o contorno possua uma única tangente no ponto $S$, tem-se o seguinte valor:

$$
u_{j}(S) \cdot \lim _{\varepsilon \rightarrow 0} \int_{\Gamma_{\varepsilon}} p_{i j}^{*}(S, Q) \cdot d \Gamma=-\frac{1}{2} \cdot \delta_{i j} \cdot u_{j}(S)
$$

O valor da integral (5.35) pode ser verificado com detalhes em Brebbia \& Dominguez (1992). 
Dessa forma a equação integral para pontos no contorno tem o seguinte aspecto:

$$
\begin{aligned}
c_{i j}(S) \cdot u_{j}(S)+\int_{\Gamma} u_{j}(Q) \cdot p_{i j}^{*}(S, Q) \cdot d \Gamma(Q)= & \int_{\Gamma} p_{j}(Q) \cdot u_{i j}^{*}(S, Q) \cdot d \Gamma(Q) \\
& +\int_{\Omega} b_{j}(q) \cdot u_{i j}^{*}(S, q) \cdot d \Omega(q)
\end{aligned}
$$

Onde:

$$
c_{i j}(S)=\frac{1}{2} \delta_{i j} \quad i, j=1,2,3
$$

Para pontos externos ao domínio pode-se obter uma expressão semelhante à (5.36), mas com o coeficiente $c_{i j}$ igual a zero. Assim a expressão (5.36) torna-se uma expressão geral cujo coeficiente $\mathrm{c}_{\mathrm{ij}}$ possui os seguintes valores:

$$
\begin{array}{ll}
c_{i j}(S)=\delta_{i j} & \mathrm{p} / \text { pontos internos } \\
c_{i j}(S)=\frac{1}{2} \cdot \delta_{i j} & \mathrm{p} / \text { pontos do contorno suave } \\
c_{i j}(S)=0 & \mathrm{p} / \text { pontos externos }
\end{array}
$$

O Método dos Elementos de Contorno tem origem com a avaliação numérica da expressão (5.36). Esta formulação é denominada de formulação direta, pois a equação (5.36) expressa deslocamentos e forças de superfície de pontos do contorno do sólido, e tais variáveis possuem significado físico imediato.

\subsection{Equação integral para a solução hiper-singular}

A equação (5.36) é válida no caso da solução fundamental de Kelvin; para utilizar a solução hiper-singular é necessário derivá-la em relação a uma direção qualquer (genericamente chamada de $\mathrm{k}$ ).

$$
\begin{aligned}
& \frac{\partial\left[c_{i j}(s) \cdot u_{j}(s)\right]}{\partial x_{k}(s)}+\frac{\partial}{\partial x_{k}(s)} \int_{\Gamma} u_{j}(Q) \cdot p_{i j}^{*}(s, Q) \cdot d \Gamma(Q)= \\
& \frac{\partial}{\partial x_{k}(s)} \int_{\Gamma} p_{j}(Q) \cdot u_{i j}^{*}(s, Q) \cdot d \Gamma(Q)+\frac{\partial}{\partial x_{k}(s)} \int_{\Omega} b_{j}(q) \cdot u_{i j}^{*}(s, q) \cdot d \Omega(q)
\end{aligned}
$$


Neste trabalho será utilizado ponto fonte externo, desta forma o significado físico das variáveis do problema é facilmente preservado, alterando-se assim apenas as funções ponderadoras presentes nas integrais de contorno.

Deve-se notar que, para cada ponto fonte exterior pode-se escolher a equação (5.40) ou a equação (5.36), tornando bastante flexível a composição da técnica a ser apresentada.

$$
\begin{aligned}
\int_{\Gamma} u_{j}(Q) \cdot \frac{\partial}{\partial x_{k}(s)} p_{i j}^{*}(s, Q) \cdot d \Gamma(Q) & =\int_{\Gamma} p_{j}(Q) \cdot \frac{\partial}{\partial x_{k}(s)} u_{i j}^{*}(s, Q) \cdot d \Gamma(Q) \\
& +\int_{\Omega} b_{j}(q) \cdot \frac{\partial}{\partial x_{k}(s)} u_{i j}^{*}(s, q) \cdot d \Omega(q)
\end{aligned}
$$

Ou seja, basta substituir a solução fundamental pela solução hiper-singular quando o ponto fonte não for ponto interno ou do contorno. 


\section{CAPÍTULO 6}

\section{MÉTODO DOS ELEMENTOS DE CONTORNO}

\subsection{Introdução}

As equações integrais de contorno servem de base ao Método dos Elementos de Contorno, mas para utilizá-las no método torna-se necessária a discretização do contorno em elementos com várias formas possíveis, transformando-as em equações algébricas, a fim de que possam ser resolvidas numericamente.

Normalmente os elementos utilizados para a discretização de um sólido tridimensional possuem a forma triangular ou quadrangular, podendo ser planos ou curvos. Estes elementos ainda podem possuir funções interpoladoras, definidas por polinômios constantes, lineares, quadráticos ou de ordem superior.

\subsection{Equacionamento algébrico}

Para se escrever o equacionamento algébrico do método é necessário inicialmente determinar as coordenadas cartesianas de um ponto $P$ qualquer de um elemento em função das coordenadas dos nós que o definem. Essas coordenadas podem ser expressas em termos de notação indicial, como segue:

$$
x_{i}=\Phi_{k} \cdot X_{i}^{k}
$$


Onde:

$\mathrm{x}_{\mathrm{i}}$ : coordenadas cartesianas do ponto $\mathrm{P}$

$\Phi_{\mathrm{k}}$ : funções interpoladoras

$X_{i}^{k}$ : coordenadas cartesianas dos nós do elemento

Que em forma matricial pode ser expressa da seguinte forma:

$x=\Phi^{T} \cdot X^{n}$

De maneira análoga são determinadas expressões para deslocamentos e forças de superfície:

$$
\begin{aligned}
& \underset{\sim}{u}=\underset{\sim}{\Phi^{T}} \cdot{\underset{\sim}{U}}^{n} \\
& p=\underset{\sim}{\Phi^{T}} \cdot P_{\sim}^{n}
\end{aligned}
$$

Da mesma forma, as forças volumétricas são dadas pela seguinte expressão:

$$
\underset{\sim}{b}=\underset{\sim}{\Phi_{c}^{T}} \cdot{\underset{\sim}{B}}^{n}
$$

Onde:

$\Phi e \Phi_{c}$ : funções interpoladoras do elemento e da célula, respectivamente.

$U^{n}$ e $P^{n}$ : valores de deslocamentos e forças de superfície nodais do elemento, respectivamente.

$B^{n}$ : valores de forças volumétricas nodais da célula.

Aplicando-se as aproximações apresentadas sobre a Identidade Somigliana (equação 5.24) para um ponto $S$ qualquer e uma discretização do contorno em $L$ elementos e uma discretização do domínio em $\mathrm{M}$ células, a seguinte equação algébrica é determinada: 


$$
\begin{aligned}
& \underset{\sim}{c(S)} \underset{\sim}{u}(S)+\sum_{i=1}^{L}\left[\int_{\Gamma_{i}} p^{*}(S, Q) \cdot{\underset{\sim}{\Phi}}^{T}(Q) \cdot d \Gamma(Q)\right] \cdot{\underset{\sim}{U}}^{N}(Q)= \\
& \sum_{i=1}^{L}\left[\int_{\Gamma_{i}} u^{*}(S, Q) \cdot{\underset{\sim}{T}}^{T}(Q) \cdot d \Gamma(Q)\right] \cdot P_{\sim}^{N}(Q) \\
& +\sum_{j=1}^{M}\left[\int_{\Omega_{j}}^{\underset{\sim}{u}}{ }^{*}(S, q) \cdot \underset{\sim c}{\Phi^{T}}(q) \cdot d \Omega(q)\right] \cdot \boldsymbol{\sim}_{\sim}^{N}(q)
\end{aligned}
$$

Onde o índice $\mathrm{N}$ nos vetores $\mathrm{U}, \mathrm{P}$ e $\mathrm{B}$ indica que se trata dos vetores com os valores de todos os elementos ou células, e não somente do elemento i ou da célula j.

Deve-se observar que uma equação semelhante a (6.6a) pode ser escrita para a equação (5.40).

$$
\begin{aligned}
& \sum_{i=1}^{L}\left[\int_{\Gamma_{i}} \frac{\partial}{\partial x_{k}(S)} p_{\sim}^{*}(S, Q) \cdot \sim_{\sim}^{\Phi^{T}}(Q) \cdot d \Gamma(Q)\right] \cdot \mathcal{\sim}^{N}(Q)= \\
& \sum_{i=1}^{L}\left[\int_{\Gamma_{i}} \frac{\partial}{\partial x_{k}(S)} u_{\sim}^{*}(S, Q) \cdot{\underset{\sim}{\sim}}^{T}(Q) \cdot d \Gamma(Q)\right] \cdot{\underset{\sim}{\sim}}^{N}(Q) \\
& +\sum_{j=1}^{M}\left[\int_{\Omega_{j}} \frac{\partial}{\partial x_{k}(S)} u_{\sim}^{*}(S, q) \cdot \underset{\sim}{\Phi_{c}^{T}}(q) \cdot d \Omega(q)\right] \cdot{\underset{\sim}{\sim}}^{N}(q)
\end{aligned}
$$

Ou ainda, tomando-se a derivada na direção normal aos elementos de contorno, esta fica:

$$
\begin{aligned}
& \sum_{i=1}^{L}\left[\int_{\Gamma_{i}} \frac{\partial}{\partial x_{k}(S)} p_{\sim}^{*}(S, Q) \cdot \eta_{k}^{i} \cdot{\underset{\sim}{\sim}}^{T}(Q) \cdot d \Gamma(Q)\right] \cdot{\underset{\sim}{U}}^{N}(Q)= \\
& \sum_{i=1}^{L}\left[\int_{\Gamma_{i}} \frac{\partial}{\partial x_{k}(S)} u_{\sim}^{*}(S, Q) \cdot \eta_{k}^{i} \cdot \underset{\sim}{\Phi^{T}}(Q) \cdot d \Gamma(Q)\right] \cdot \underset{\sim}{P^{N}}(Q) \\
& +\sum_{j=1}^{M}\left[\int_{\Omega_{j}} \frac{\partial}{\partial x_{k}(S)} u_{\sim}^{*}(S, q) \cdot \eta_{k}^{i} \cdot \underset{\sim}{\Phi_{c}^{T}}(q) \cdot d \Omega(q)\right] \cdot{\underset{\sim}{\sim}}^{N}(q)
\end{aligned}
$$

Tomando-se o número de pontos fonte igual ao número de nós do contorno, escolhendo-se convencionalmente, para cada ponto fonte, a equação (6.6a) ou (6.6c) pode-se escrever matricialmente:

$$
\underset{\sim}{C} \cdot \underset{\sim}{U}+\underset{\sim}{H} \cdot \underset{\sim}{U}=\underset{\sim}{G} \cdot \underset{\sim}{P}+\underset{\sim}{D} \cdot \underset{\sim}{B}
$$


Onde:

$H, G$ e $D$ : matrizes determinadas através das integrais numéricas sobre os elementos e células, e $C$ é a matriz dos termos livres dados em (5.38) para as linhas referentes àequação (6.6a).

Adicionando-se a matriz $C$ àmatriz $\hat{H}$, obtém-se:

$$
\underset{\sim}{H} \cdot \underset{\sim}{U}=\underset{\sim}{G} P \underset{\sim}{P}+\underset{\sim}{D} \cdot \underset{\sim}{B}
$$

Este é o sistema algébrico de equações para a solução do problema elástico bi ou tridimensional, utilizando-se soluções fundamentais de Kelvin (singulares) e suas derivadas (hiper-singulares).

Conhecendo-se os valores prescritos dos deslocamentos e forças de superfície de alguns pontos do contorno e os valores das forças volumétricas, devese trocar as colunas das matrizes $\mathrm{He} \mathrm{G}$ e os respectivos valores de deslocamentos e forças de superfície de modo que todas as incógnitas do problema fiquem do lado esquerdo da igualdade, tendo-se com isso um sistema algébrico que pode ser resolvido com qualquer procedimento para resolução de sistema linear:

$$
\underset{\sim}{A} \underset{\sim}{X}=\underset{\sim}{F}
$$

Onde:

A: matriz cujas colunas correspondem a valores incógnitos; é válido lembrar que esta matriz é sempre cheia e não-simétrica.

$X$ : vetor que contém as incógnitas, que são deslocamentos e forças de superfície.

$F$ : vetor obtido através da multiplicação da matriz $\mathrm{G}$, com os valores já trocados, e o vetor $\mathrm{P}$, que possui apenas valores conhecidos de deslocamentos e forças de superfície. Este ainda pode ser acrescido da contribuição das forças de volume $D \cdot B$. 


\subsection{Propriedades geométricas dos elementos}

A discretização da superfície dos sólidos tridimensionais será feita através de elementos triangulares planos, como pode ser visto na figura 6.1.

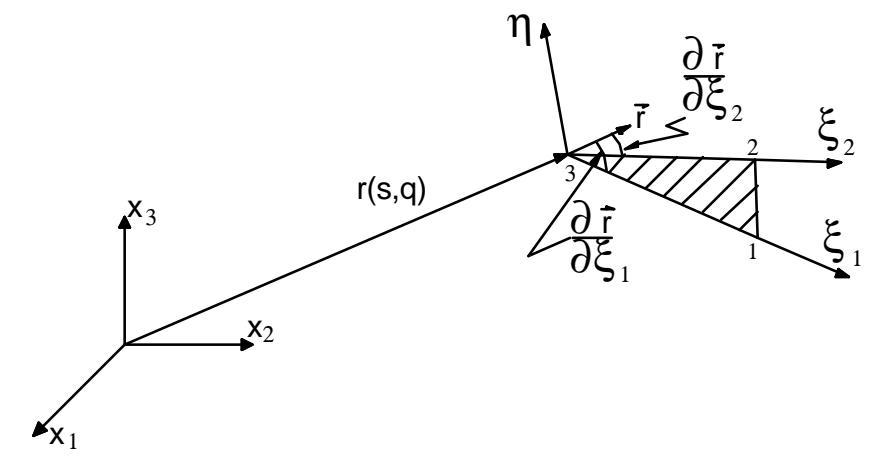

Figura 6.1 - Sistema de coordenadas locais e globais

Considerando-se os sistemas locais e globais definidos na figura 6.1, a matriz que relaciona a diferenciação de uma função qualquer em coordenadas globais e tal diferenciação em coordenadas locais é dada pela seguinte expressão:

$$
\left\{\begin{array}{l}
\frac{\partial \vec{r}}{\partial \xi_{1}} \\
\frac{\partial \vec{r}}{\partial \xi_{2}} \\
\frac{\partial \vec{r}}{\partial \xi_{3}}
\end{array}\right\}=\left[\begin{array}{lll}
\frac{\partial x_{1}}{\partial \xi_{1}} & \frac{\partial x_{2}}{\partial \xi_{1}} & \frac{\partial x_{3}}{\partial \xi_{1}} \\
\frac{\partial x_{1}}{\partial \xi_{2}} & \frac{\partial x_{2}}{\partial \xi_{2}} & \frac{\partial x_{3}}{\partial \xi_{2}} \\
\frac{\partial x_{1}}{\partial \eta} & \frac{\partial x_{2}}{\partial \eta} & \frac{\partial x_{3}}{\partial \eta}
\end{array}\right] \cdot\left\{\begin{array}{l}
\frac{\partial \vec{r}}{\partial x_{1}} \\
\frac{\partial \vec{r}}{\partial x_{2}} \\
\frac{\partial \vec{r}}{\partial x_{3}}
\end{array}\right\}=J \cdot\left\{\begin{array}{l}
\frac{\partial \vec{r}}{\partial x_{1}} \\
\frac{\partial \vec{r}}{\partial x_{2}} \\
\frac{\partial \vec{r}}{\partial x_{3}}
\end{array}\right\}
$$

Onde:

$J$ : Matriz Jacobiana de transformação entre o sistema de coordenadas globais e o sistema de coordenadas locais.

O diferencial do volume $\mathrm{d} \Omega$ é dado por:

$$
d \Omega=\left|\frac{\partial \vec{r}}{\partial \xi_{1}} \times \frac{\partial \vec{r}}{\partial \xi_{2}} \cdot \frac{\partial \vec{r}}{\partial \eta}\right| \cdot d \xi_{1} \cdot d \xi_{2} \cdot d \eta=|J| \cdot d \xi_{1} \cdot d \xi_{2} \cdot d \eta
$$


E o diferencial da área $\mathrm{d} \Gamma$ é dado por:

$$
d \Gamma=\left|\frac{\partial \vec{r}}{\partial \xi_{1}} \times \frac{\partial \vec{r}}{\partial \xi_{2}}\right| \cdot d \xi_{1} \cdot d \xi_{2}=|\underset{\sim}{G}| \cdot d \xi_{1} \cdot d \xi_{2}
$$

Para o elemento triangular plano com aproximação linear da geometria o jacobiano de transformação é constante e numericamente igual ao dobro da área do elemento:

$$
|\underset{\sim}{G}|=2 \cdot A
$$

As coordenadas locais quando tratadas de forma adimensional, ou seja, assumindo valores de 0 a 1 , são chamadas de coordenadas homogêneas (ver figura 6.2).
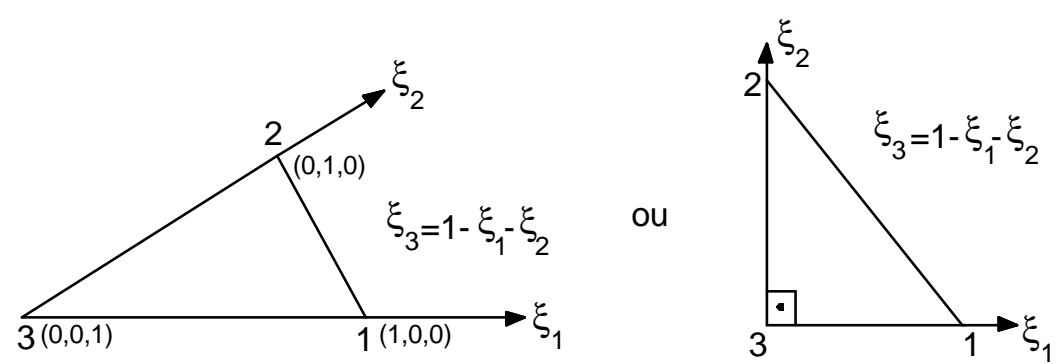

Figura 6.2 - Coordenadas homogêneas

As coordenadas homogêneas servem para definir as coordenadas dos pontos, pois sendo:

$$
\underset{\sim}{\Phi}=f\left(\xi_{1}, \xi_{2}, \xi_{3}\right)
$$

De acordo com (6.2), tem-se para as funções de interpolação constante e linear e quadrática:

$$
x_{i}=\ddot{O}^{j} \cdot X_{i}^{j}
$$

Onde:

j=1, 2 e 3 para as funções de interpolação constante e linear e j=1, 2, 3, 4, 5 e 6 para a função de interpolação quadrática. 
$X_{i}^{j}$ : são as coordenadas dos nós do elemento a ser integrado, sendo o índice i referente àdireção da coordenada e o índice j referente ao nó.

\subsection{Funções de interpolação}

As funções de interpolação servem para aproximar a solução das variáveis envolvidas no problema. Essa aproximação se dá em forma de interpolação dos valores nodais. As funções de interpolação são formadas por polinômios e podem ser constantes, lineares, quadráticas, cúbicas ou de ordens mais elevadas. Neste trabalho tratar-se-á apenas com funções de interpolação constante, linear e quadrática.

\subsubsection{Função de interpolação constante}

Para essa função de interpolação todo o elemento possui os mesmos valores de deslocamentos e forças de superfície, então, para o elemento triangular, o único valor que precisa ser avaliado é o valor do ponto centroidal do elemento. A função de aproximação $\Phi$ recebe o valor de 1, como pode ser visto na figura 6.3.

A vantagem do elemento com a função de interpolação constante é que pode ser aplicado sobre qualquer problema que envolva descontinuidade, mas possui a desvantagem de que para se conseguir aumentar a precisão de qualquer modelo é necessário um grande refinamento da malha.

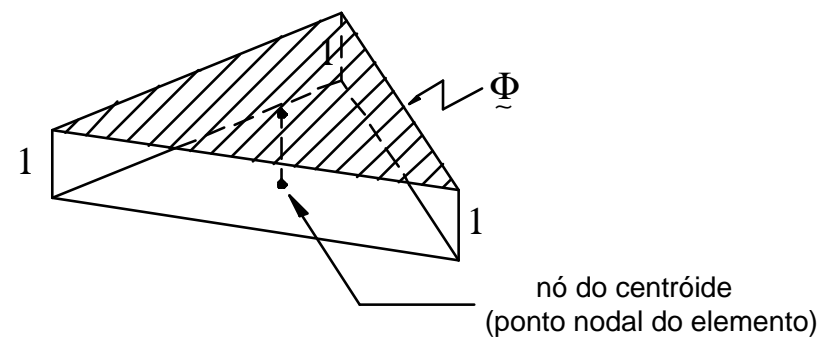

Figura 6.3 - Elemento triangular constante

Sendo $\Phi^{T}$ a transposta de $\Phi$ e dada pela seguinte matriz: 


$$
\tilde{\Phi}^{T}=\left[\begin{array}{lll}
1 & 0 & 0 \\
0 & 1 & 0 \\
0 & 0 & 1
\end{array}\right]
$$

E de acordo com as equações (6.3) e (6.4), os deslocamentos e forças de superfície dos pontos pertencentes ao elemento a ser integrado, serão dados por:

$$
\begin{aligned}
& \left\{\begin{array}{l}
u_{1} \\
u_{2} \\
u_{3}
\end{array}\right\}=\left[\begin{array}{lll}
1 & 0 & 0 \\
0 & 1 & 0 \\
0 & 0 & 1
\end{array}\right] \cdot\left\{\begin{array}{l}
U_{1}^{1} \\
U_{2}^{1} \\
U_{3}^{1}
\end{array}\right\} \\
& \left\{\begin{array}{l}
p_{1} \\
p_{2} \\
p_{3}
\end{array}\right\}=\left[\begin{array}{lll}
1 & 0 & 0 \\
0 & 1 & 0 \\
0 & 0 & 1
\end{array}\right] \cdot\left\{\begin{array}{l}
P_{1}^{1} \\
P_{2}^{1} \\
P_{3}^{1}
\end{array}\right\}
\end{aligned}
$$

Onde:

$\mathrm{u}_{\mathrm{i}}$ e $\mathrm{p}_{\mathrm{i}}$ : deslocamentos e forças de superfície nas direções 1, 2 e 3.

$U_{i}^{1}$ e $P_{i}^{1}$ : deslocamentos e forças de superfície no centróide do elemento, o índice i se refere às coordenadas 1,2 e 3.

\subsubsection{Função de interpolação linear}

A função de interpolação linear permite uma melhor aproximação das variáveis do que a função constante. Para o elemento triangular ela consiste em interpolar linearmente entre os três valores nodais do elemento.

No elemento triangular linear os nós do elemento são os próprios nós dos vértices do elemento, e as variáveis são aproximadas com funções de interpolação $\Phi^{1}, \Phi^{2}$ e $\Phi^{3}$, que assumem valores unitários no respectivo nó do elemento e zero no lado oposto ao nó, conforme mostrado na figura 6.4. 

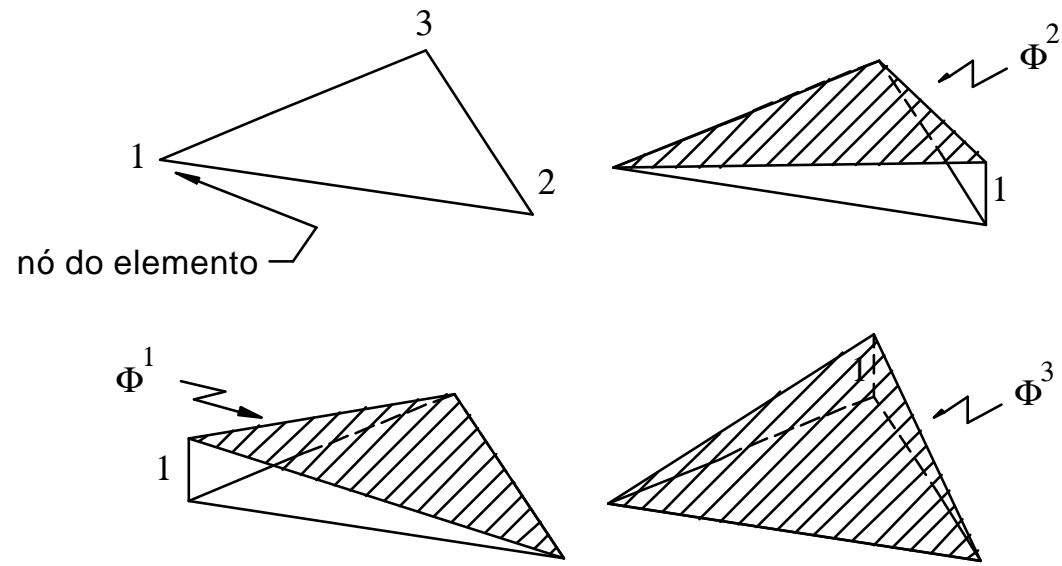

Figura 6.4 - Elemento triangular linear

Utilizando-se das coordenadas homogêneas $\left(\xi_{1}, \xi_{2}\right.$ e $\left.\xi_{3}\right)$, já apresentadas anteriormente, tem-se que para um ponto qualquer do elemento:

$$
\begin{aligned}
& \Phi^{1}=\xi_{1} \\
& \Phi^{2}=\xi_{2} \\
& \Phi^{3}=\xi_{3}
\end{aligned}
$$

Os valores de deslocamentos e forças de superfície são obtidos analogamente àexpressão (6.15), onde se mostra a obtenção das coordenadas.

\subsubsection{Função de interpolação quadrática}

A função de interpolação quadrática permite uma melhor aproximação das variáveis do que as funções apresentadas anteriormente.

No elemento triangular quadrático os nós do elemento são os próprios nós dos vértices do elemento e os pontos médios de cada lado do triângulo, como pode ser visto na figura 6.5, e as variáveis são aproximadas com funções de interpolação $\Phi^{1}, \Phi^{2}, \Phi^{3}, \Phi^{4}, \Phi^{5}$ e $\Phi^{6}$ apresentadas na equação (6.19). 


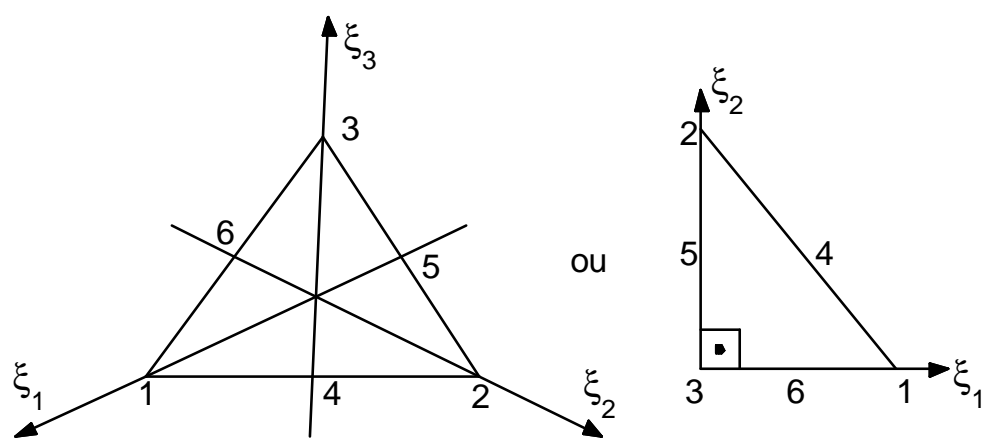

Figura 6.5 - Nós do elemento quadrático

$$
\begin{aligned}
& \Phi^{1}=\xi_{1} \cdot\left(2 \cdot \xi_{1}-1\right) \\
& \Phi^{2}=\xi_{2} \cdot\left(2 \cdot \xi_{2}-1\right) \\
& \Phi^{3}=\xi_{3} \cdot\left(2 \cdot \xi_{3}-1\right) \\
& \Phi^{4}=4 \cdot \xi_{1} \cdot \xi_{2} \\
& \Phi^{5}=4 \cdot \xi_{2} \cdot \xi_{3} \\
& \Phi^{6}=4 \cdot \xi_{3} \cdot \xi_{1}
\end{aligned}
$$

Os valores de deslocamentos e forças de superfície são obtidos analogamente àexpressão (6.15).

Para o tratamento de descontinuidade de forças de superfície não é necessário aplicar os conceitos de elementos contínuos e descontínuos. Quando se desejam duas equações diferentes para uma mesma posição geométrica basta apenas utilizar-se dois números de nós para a mesma posição geométrica. Caso existam dois pontos fontes singulares (sobre elementos adjacentes) o termo livre $c_{i j}$ será colocado em colunas diferentes da matriz $\mathrm{H}$, devido à numeração dos nós, garantindo a não singularidade da matriz $A$, desde que os dois nós referidos não estejam com deslocamentos restritos, o que causaria a alocação de termos idênticos da matriz $G$ nas respectivas colunas da matriz $A$, brnando-a singular. Neste caso adotam-se dois pontos fontes exteriores para canto ou um singular $e$ outro exterior àsuperfície plana. 


\subsection{Processos de integração}

\subsubsection{Integral não singular}

A integral não singular ocorre quando o ponto fonte não pertence ao elemento a ser integrado ou quando o ponto fonte não está muito próximo ao elemento.

A integração não singular é a forma mais direta de se calcular a integral sobre um elemento. No caso do elemento triangular plano com aproximações constante, linear ou quadrática, a integração é feita através da quadratura de Hammer et al. (1956), podendo facilmente ser encontrada em tabelas tal como em Brebbia \& Dominguez (1992) e é estabelecida em função das coordenadas homogêneas.

As componentes das matrizes H e G são obtidas através da integração sobre cada elemento do contorno, conforme apresentado nas equações (6.6a-c), onde:

$$
\begin{aligned}
& \underset{\sim}{g}=\int_{\Gamma_{j}} u_{\sim}^{*}(S, Q) \cdot \Phi_{\sim}^{T}(Q) \cdot d \Gamma(Q) \\
& \underset{\sim}{h}=\int_{\Gamma_{j}} p^{*}(S, Q) \cdot{\underset{\sim}{\Phi}}^{T}(Q) \cdot d \Gamma(Q)
\end{aligned}
$$

Para o caso em que as integrais (6.20) e (6.21) forem efetuadas numericamente através da quadratura de Hammer é preciso realizar uma transformação de coordenadas globais para coordenadas homogêneas.

$$
\underset{\sim}{g}=|G| \cdot \int_{0}^{1}\left(\int_{0}^{1-\xi_{2}} f\left(\xi_{1}, \xi_{2}, \xi_{3}\right) \cdot d \xi_{1}\right) \cdot d \xi_{2}
$$

E só então aplicar a quadratura em forma de somatório:

$$
\underset{\sim}{g}=|G| \cdot \sum_{i=1}^{n} f_{\sim}\left(\xi_{1}^{i}, \xi_{2}^{i}, \xi_{3}^{i}\right) \cdot w_{i}
$$

Onde:

$\mathrm{n}$ : número de pontos de Hammer

|G|: jacobiano de transformação 
$\xi_{1}^{i}, \xi_{2}^{i}, \xi_{3}^{i}:$ coordenadas homogêneas do ponto a ser considerado

$\mathrm{w}_{\mathrm{i}}$ : peso do ponto a ser considerado

$f\left(\xi_{1}^{i}, \xi_{2}^{i}, \xi_{3}^{i}\right)$ : integrando de (6.20) calculado no ponto i

$\sim g$

De forma análoga tem-se para a integral (6.21):

$$
\underset{\sim}{h}=|G| \cdot \sum_{i=1}^{n} \underset{\sim h}{f}\left(\xi_{1}^{i}, \xi_{2}^{i}, \xi_{3}^{i}\right) \cdot w_{i}
$$

Neste trabalho optou-se por subdividir o elemento a ser integrado em vários triângulos menores, de tal forma que os elementos mais próximos do ponto fonte sejam mais subdivididos e os elementos mais distantes utilizem uma menor subdivisão ou até nem sejam subdivididos. Esta subdivisão é feita comparando-se a distância em que o ponto fonte está do elemento a ser integrado com a média dos lados deste elemento, neste trabalho optou-se por usar a distância do nó mais próximo e não do centróide do elemento. O número de subelementos é calculado através do fator multiplicador, que é um dado de entrada do programa computacional, esta subdivisão pode ser vista na figura 6.6.

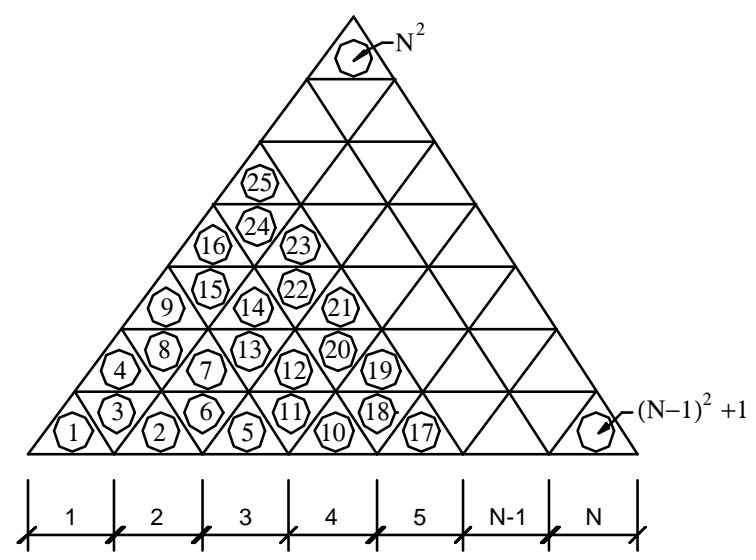

Figura 6.6 - Subdivisão do elemento triangular plano

Esta subdivisão é baseada na subdivisão apresentada por Calderón (1996), o qual utiliza no máximo 25 subelementos. No atual trabalho a subdivisão é feita em um número qualquer de subelementos. Esta técnica é utilizada em diversos trabalhos para considerar inclusive a integração de pontos muito próximos aos 
elementos, chamados quase singulares. Porém neste trabalho foi verificada sua baixa eficiência quando utilizada para esta finalidade.

A determinação do número máximo de subelementos é feita da seguinte forma:

Quando o ponto fonte S pertence inicialmente ao elemento integrado tem-se $\mathrm{F}$ como fator multiplicador que coloca o ponto para fora $(0.1 \leq \mathrm{F} \leq 0.5)$, o número máximo de subdivisões de cada lado é dado pela seguinte regra:

$$
N=\operatorname{int}\left(\sqrt{\frac{10}{F}}\right)
$$

$\mathrm{N}$ é o valor inteiro mais próximo da raiz quadrada anterior. Esta regra foi obtida no sentido de satisfazer a precisão necessária à integração, que possui singularidade $\left(1 / r^{2}\right)$ e, além disso, concordar com a apresentada por Calderón (1996) para o caso em que $\mathrm{F}=0.4$.

Dessa forma, o número de subelementos é dado por:

$$
\begin{array}{lcl}
0 \leq D \leq \bar{L} & N^{2} & \text { subelements } \\
\bar{L}<D \leq 2 \bar{L} & (N-3)^{2} & \text { subelemenos } \\
2 \bar{L}<D & 1 & \text { subelemeno }
\end{array}
$$

Onde:

D: distância do ponto fonte ao nó mais próximo pertencente ao elemento a ser integrado

$\bar{L}:$ média dos lados do elemento a ser integrado

Dessa forma os somatórios (6.23) e (6.24) assumem a seguinte expressão para um número NS de subelementos:

$$
\begin{aligned}
& \underset{\sim}{g}=\sum_{M=1}^{N S} \frac{|G|}{N S} \cdot \sum_{i=1}^{n} \underset{\sim g}{f}\left(\xi_{1}^{i}, \xi_{2}^{i}, \xi_{3}^{i}\right) \cdot w_{i} \\
& \underset{\sim}{h}=\sum_{M=1}^{N S} \frac{|G|}{N S} \cdot \sum_{i=1}^{n} \underset{\sim h}{f}\left(\xi_{1}^{i}, \xi_{2}^{i}, \xi_{3}^{i}\right) \cdot w_{i}
\end{aligned}
$$




\subsubsection{Integral quase singular e quase hiper-singular}

Para se efetuar as integrais quando o ponto está muito próximo ao elemento e a utilização da quadratura de Hammer torna-se muito dispendiosa com relação ao tempo de processamento (número de subelementos muito grande para se obter resultados aceitáveis) é preciso recorrer a um método alternativo para avaliar essa integral de forma mais rápida, baseado no trabalho de Mom-Ma et al. (1996), que efetua a integração em elementos unidimensionais através da quadratura de Gauss $^{1}$ com subdivisão progressiva dos elementos. Esta técnica foi adaptada para elementos triangulares planos conforme mostra a figura 6.7. Considerando-se primeiramente o ponto fonte no contorno, utiliza-se uma transformação das coordenadas cartesianas para coordenadas polares e para cada ângulo $\theta$ subdivide-se o elemento radialmente, pois este se torna unidimensional no raio.

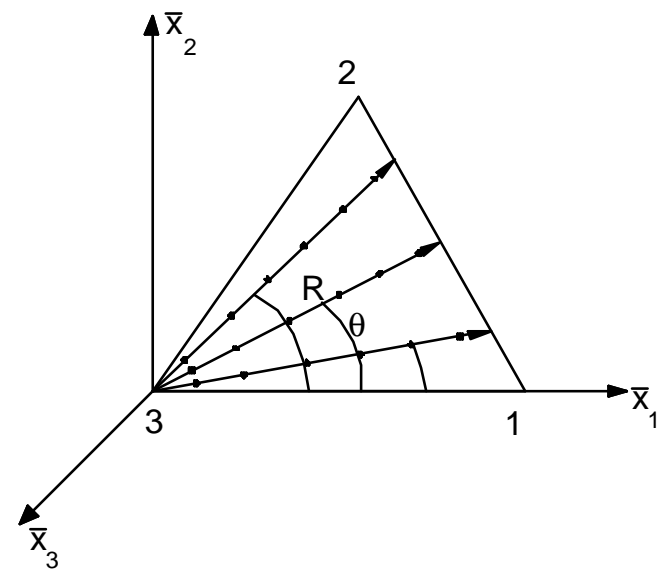

Figura 6.7 - Esquema para integração quase singular para o elemento linear

Com o interesse de generalizar essa integração é efetuada outra mudança de coordenadas, agora para coordenadas homogêneas, conforme a figura 6.8.

\footnotetext{
${ }^{1}$ Stroud, A. H., and Secrest, D. (1966). Gaussian Quadrature Formulas. Prentice Hall, New York.
} 


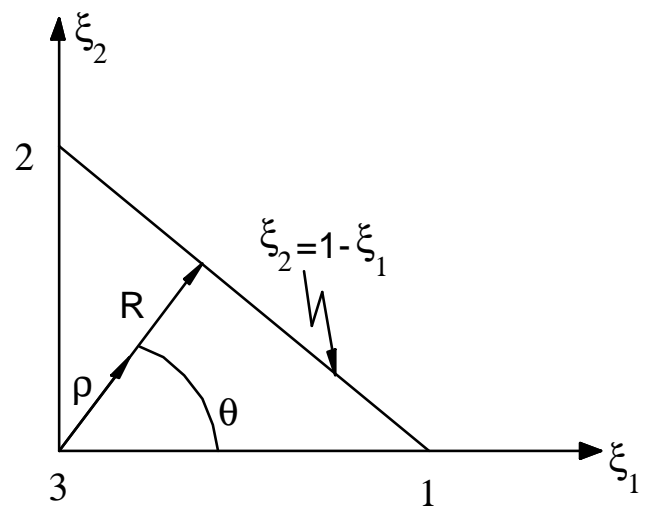

Figura 6.8 - Transformação para coordenadas homogêneas

Onde:

$\mathrm{R}$ : raio adimensional limite superior

$\rho$ : raio adimensional

$\theta$ : ângulo entre o raio e o eixo $\xi_{1}$

Da figura 6.8 tiram-se as seguintes expressões:

$\xi_{1}=\rho \cdot \cos (\theta)$

$\xi_{2}=\rho \cdot \operatorname{sen}(\theta)$

$R(\theta)=\frac{1}{\operatorname{sen}(\theta)+\cos (\theta)}$

Dessa forma a integral (6.22) torna-se:

$\underset{\sim}{g}=|G| \cdot \int_{0}^{\frac{\pi}{2}} \int_{0}^{R(\theta)} f\left(\xi_{1}, \xi_{2}, \xi_{3}\right) \cdot \rho \cdot d \rho \cdot d \theta$

Transformando a variação radial para coordenadas adimensionais:

$\underset{\sim}{g}=|G| \cdot \int_{0}^{\frac{\pi}{2}} \int_{-1}^{1} \frac{R(\theta)}{2} \cdot \underset{\sim g}{f}\left(\xi_{1}, \xi_{2}, \xi_{3}\right) \cdot \rho(\eta) \cdot d \eta \cdot d \theta$

Onde $\eta$ é a coordenada adimensional radial (figura 6.9): 


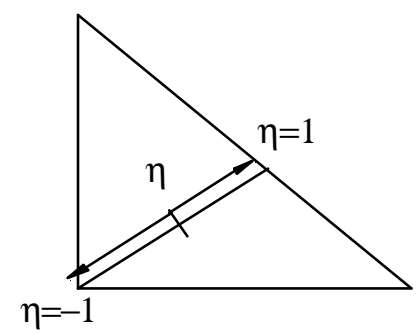

Figura 6.9 - Coordenada adimensional radial

Transformando a variação angular para coordenadas adimensionais:

$$
\underset{\sim}{g}=|G| \cdot \int_{-1}^{1} \int_{-1}^{1} \frac{R[\theta(\gamma)]}{2} \cdot \frac{\pi}{4} \cdot \underset{\sim g}{f}\left(\xi_{1}, \xi_{2}, \xi_{3}\right) \cdot \rho(\eta) \cdot d \eta \cdot d \gamma
$$

Onde g é a coordenada adimensional angular (figura 6.10):

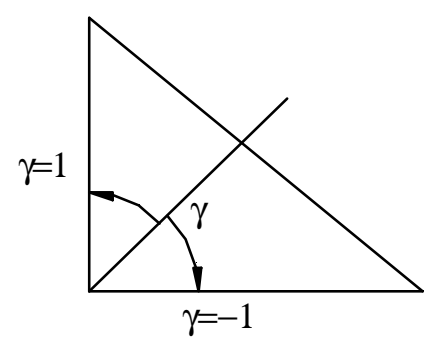

Figura 6.10 - Coordenada adimensional angular

Aplicando-se a quadratura de Gauss, a integral (6.34) assume a seguinte forma de somatório:

$$
\underset{\sim}{g}=|G| \cdot \frac{\pi}{4} \cdot \sum_{i \theta=1}^{i g \gamma} \frac{R[\theta(\gamma)]}{2} \cdot \sum_{i r=1}^{i g r} \underset{\sim}{f}\left(\xi_{1}, \xi_{2}, \xi_{3}\right) \cdot \rho(\eta) \cdot w_{i \theta} \cdot w_{i r}
$$

Onde:

$|G|$ : Jacobiano de transformação, no caso do elemento triangular plano vale numericamente o dobro da área do elemento.

lgr: número de pontos de Gauss para a variação do ângulo

igr: número de pontos de Gauss para a variação do raio

$\mathrm{R}[\theta(\gamma)]$ : extensão máxima do raio para a posição $\theta(\gamma)$

$f$ : integrando da integral (6.21)

$\sim g$

$\rho(\eta)$ : raio do ponto de Gauss 
$\mathrm{w}_{\mathrm{i} \theta}$ : peso dos pontos de Gauss para a variação angular

$\mathrm{w}_{\mathrm{ir}}$ : peso dos pontos de Gauss para a variação radial

De forma análoga, tem-se para a integral (6.21):

$$
\underset{\sim}{h}=|G| \cdot \frac{\pi}{4} \cdot \sum_{i \theta=1}^{i g \gamma} \frac{R[\theta(\gamma)]}{2} \cdot \sum_{i r=1}^{i g r} \underset{\sim h}{f}\left(\xi_{1}, \xi_{2}, \xi_{3}\right) \cdot \rho(\eta) \cdot w_{i \theta} \cdot w_{i r}
$$

Onde:

$$
\begin{aligned}
& \theta(\gamma)=\frac{\pi}{4} \cdot(\gamma+1) \\
& R[\theta(\gamma)]=\frac{1}{\sin [\theta(\gamma)]+\cos [\theta(\gamma)]} \\
& \rho(\eta)=\frac{R[\theta(\gamma)]}{2} \cdot(\eta+1)
\end{aligned}
$$

Nas expressões acima ainda não se efetuou a subdivisão de elemento no raio. Para o caso de subdivisão calcula-se de acordo com a figura 6.11.

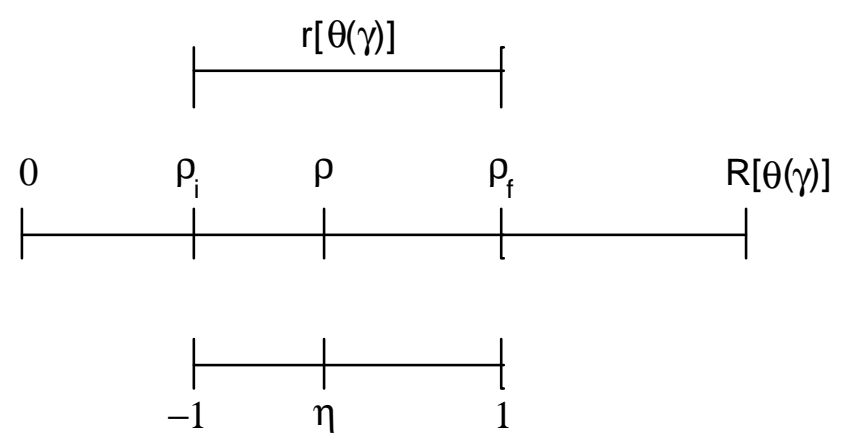

Figura 6.11 - Subelemento do raio $\mathrm{R}[\boldsymbol{\theta}(\gamma)]$

Substituindo-se $r[\theta(\gamma)]$ no lugar de $\mathrm{R}[\theta(\gamma)]$ nas expressões (6.35)-(6.39) efetuase a integração em um subelemento de raio $r$.

Para um subelemento qualquer o raio adimensional $\rho$ é dado por: 


$$
\rho[\theta(\gamma)]=\frac{(1-\eta)}{2} \cdot \rho_{i}+\frac{(1+\eta)}{2} \cdot \rho_{f}
$$

E a extensão do subelemento é dada por:

$$
r[\theta(\gamma)]=\rho_{f}-\rho_{i}
$$

E ainda:

$$
\rho_{f}=\rho_{i}+\left(\frac{d}{L}\right)^{k} \cdot m^{(n-1)}
$$

Onde, conforme Mom-Ma et al. (1996), tem-se:

$\mathrm{k}$ : ordem de quase singularidade ou quase hiper-singularidade

m: parâmetro de progressão geométrica (relação entre o tamanho do subelemento atual e o tamanho do subelemento anterior)

$\mathrm{n}$ : número do subelemento

d: distância do ponto fonte ao nó mais próximo pertencente ao elemento

L: média dos lados do elemento triangular

$\rho_{\mathrm{i}}$ assume o valor de zero para o primeiro subelemento

Assim as coordenadas homogêneas podem ser calculadas da seguinte forma:

$$
\begin{aligned}
& \xi_{i}=\rho \cdot \cos (\theta) \\
& \xi_{j}=\rho \cdot \sin (\theta) \\
& \xi_{k}=1-\xi_{i}-\xi_{j}
\end{aligned}
$$

Com:

$\mathrm{i}=1,2,3 ; \mathrm{j}=2,3,1$ e $\mathrm{k}=3,1,2$.

Onde os índices i,j e k se referem aos nós mostrados na figura 6.12. 


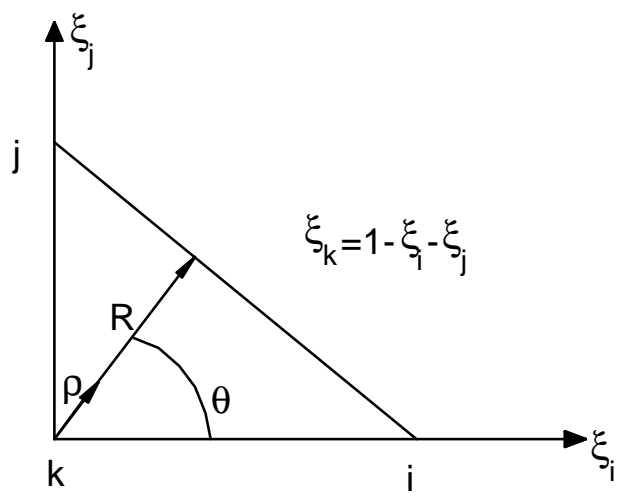

Figura 6.12 - Coordenadas homogêneas generalizadas

Finalmente, somando-se o resultado das integrações sobre cada subelemento de raio $r$ e efetuando-se o somatório de Gauss em $\theta$ resulta o valor total da integral procurada.

Pode-se utilizar esta integração para a técnica do ponto fora, para isso verifica-se qual o nó, pertencente ao elemento a ser integrado, mais próximo do ponto fonte, este será o nó k, e procede-se àintegração normalmente.

Vale lembrar ainda que esta integração é auto-adaptativa, ou seja, o tamanho dos elementos e a quantidade de pontos de Gauss variam de acordo com a distância do subelemento ao nó k.

Com a implementação desta técnica pôde-se ampliar o intervalo do fator multiplicador do ponto fora, que era $0.1 \leq \mathrm{F} \leq 0.5$ e passou a ser $0.01 \leq \mathrm{F} \leq 0.5$, apesar de 0 autor recomendar que seja utilizado $0.01 \leq F \leq 0.2$. Deve-se mencionar que o limite superior não está relacionado a problemas de integração e sim ao condicionamento do sistema que piora muito para $\mathrm{F}>0.5$.

A técnica demonstrada foi desenvolvida para o elemento linear, para utilizá-la no elemento constante basta dividir o triângulo em três partes, como mostra a figura 6.13 e proceder de maneira análoga. 


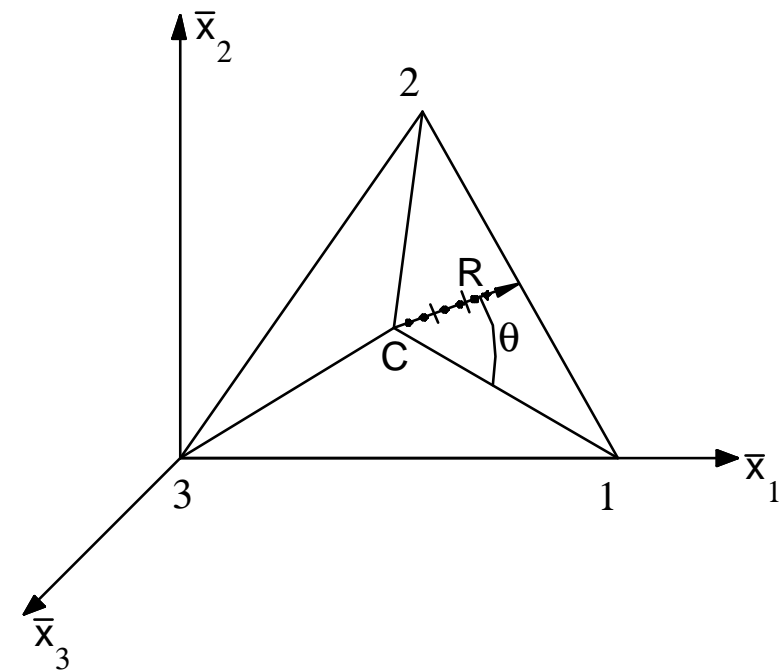

Figura 6.13 - Divisão do elemento constante para a integral quase singular

Para utilizar esta técnica no elemento quadrático, também se utiliza uma divisão do triângulo, só que em dois; como é apresentado na figura 6.14.

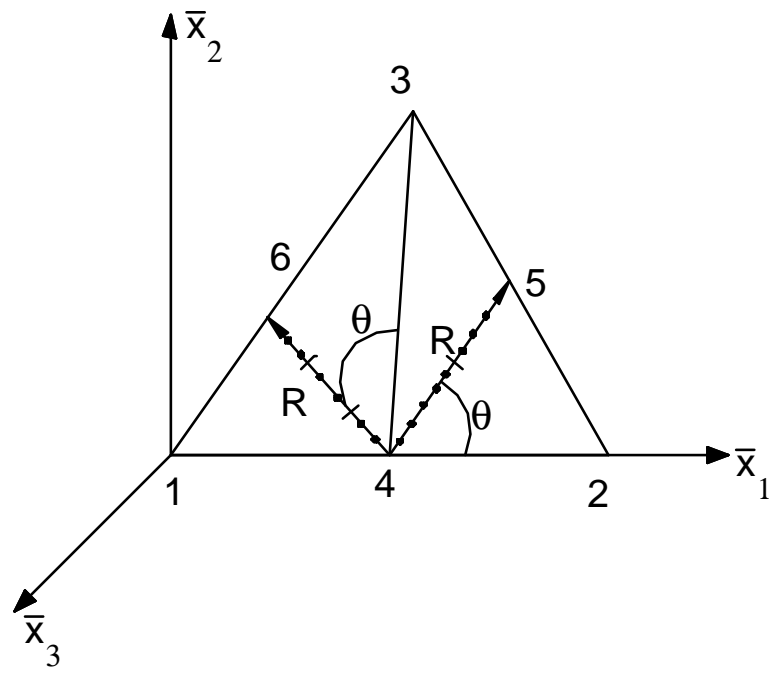

Figura 6.14 - Divisão do elemento quadrático para a integral quase singular

\subsubsection{Integral singular}

Quando o ponto fonte pertence ao elemento a ser integrado e este ponto está contido no contorno diz-se que essa integral é uma integral singular, pois a solução 
fundamental possui termos $\left(1 / \mathrm{r}\right.$ e $\left.1 / \mathrm{r}^{2}\right)$ que não podem ser integrados diretamente pelas técnicas descritas anteriormente. Para se efetuar tal integral é preciso recorrer àmudança das coordenadas cartesianas para coordenadas polares.

A seguir é apresentada a integral singular para o elemento constante, como pode ser visto na figura 6.15 , através de integração analítica e semi-analítica; já para os elementos linear e quadrático, optou-se por realizar esta integral apenas de forma semi-analítica.

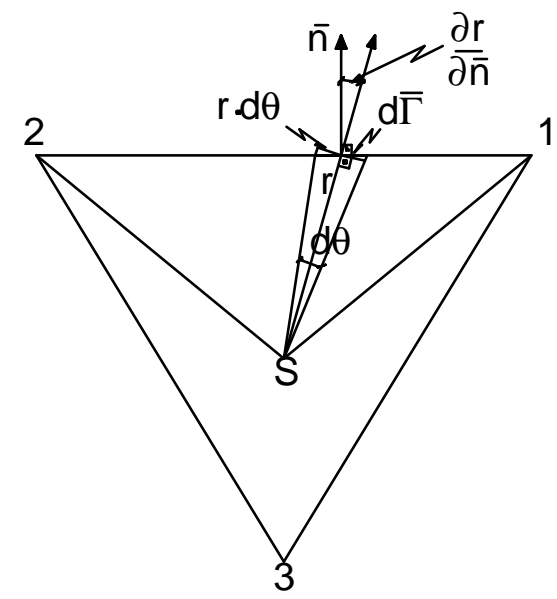

Figura 6.15 - Integral singular sobre o elemento constante

A integral sobre este elemento pode ser resolvida de três formas:

- Analiticamente em r e numericamente com a quadratura de Gauss em $\theta$;

- Analiticamente em r e com uma transformação da parte angular para linear no contorno de cada lado do elemento e efetuando-se essa parte numericamente com a quadratura de Gauss;

- Analiticamente em re $\theta$.

As duas primeiras formas denominam-se integrais semi-analíticas, pois parte da integral é resolvida analiticamente e a outra parte numericamente através da quadratura de Gauss. Neste trabalho a integral semi-analítica segue o segundo procedimento. Utilizando-se as integrais (6.20) e (6.21) e efetuando a mudança de coordenadas cartesianas para polares, tem-se que:

$$
\underset{\sim}{g}=\int_{\theta} \int_{r}^{u_{\sim}^{*}}(S, Q) \cdot \underset{\sim}{\Phi^{T}}(Q) \cdot r \cdot d r \cdot d \theta
$$




$$
\underset{\sim}{h}=\int_{\theta} \int_{r} p_{\sim}^{*}(S, Q) \cdot \underset{\sim}{\Phi^{T}}(Q) \cdot r \cdot d r \cdot d \theta
$$

Substituindo-se a solução fundamental na integral (6.44) e integrando-se primeiramente em $r$, depois mudando a variável em $\theta$ (teta) para a variável sobre 0 lado do elemento como pode ser visto na figura 6.16 e mostrado na equação (6.46), obtém-se a integral (6.47).

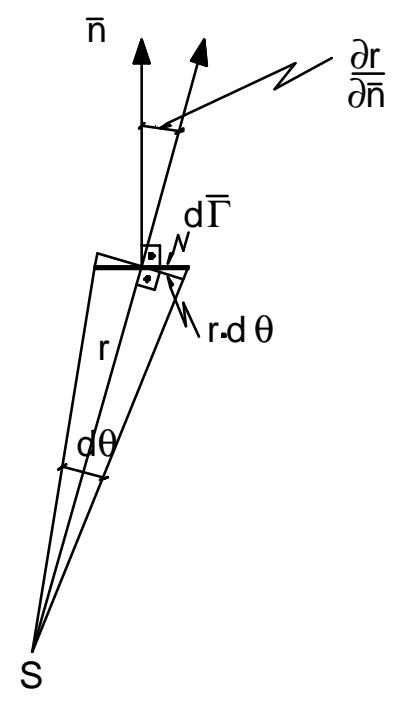

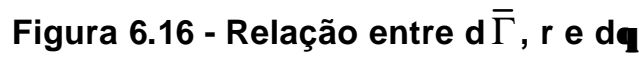

$$
\begin{aligned}
& d \bar{\Gamma} \cdot \frac{\partial r}{\partial \bar{n}}=r \cdot d \theta \\
& g_{i j}=\int_{0}^{L_{i}} \frac{1}{16 \cdot \pi \cdot G \cdot(1-v)} \cdot\left[(3-4 \cdot v) \cdot \delta_{i j}+r,,_{i} \cdot r, j\right] \cdot \frac{\partial r}{\partial \bar{n}} \cdot d \bar{\Gamma}
\end{aligned}
$$

Aplicando-se a quadratura de Gauss linearmente em cada lado do elemento, obtém-se o seguinte somatório:

$$
g_{i j}=\frac{L_{i}}{2} \cdot \sum_{i g=1}^{i g t} \frac{1}{16 \cdot \pi \cdot G \cdot(1-v)} \cdot\left[(3-4 \cdot v) \cdot \delta_{i j}+r,{ }_{i} \cdot r, j\right] \cdot \frac{\partial r}{\partial n} \cdot w_{i g}
$$

Onde:

$L_{i}$ : lado sobre o qual está integrando 
igt: número de pontos de Gauss em cada lado

$\mathrm{w}_{\mathrm{ig}}$ : peso do ponto de Gauss

$\frac{\partial r}{\partial \bar{n}}$ : derivada parcial do raio em relação ànormal ao lado.

Analogamente, o valor principal de Cauchy, pois a integral (6.21) é singular em $r$, é dado pelo seguinte somatório para a integral (6.21):

$$
h_{i j}=\frac{L_{i}}{2} \cdot \sum_{i g=1}^{i g t} \frac{(1-2 \cdot v)}{8 \cdot \pi \cdot(1-v)} \cdot\left[\left(r_{i} \cdot \eta_{j}-r,{ }_{j} \cdot \eta_{i}\right)\right] \cdot \frac{\ln (r)}{r} \cdot \frac{\partial r}{\partial n} \cdot w_{i g}
$$

Para se efetuar a integral analiticamente, segue-se exatamente os mesmos passos iniciais da anterior, só que ao invés de transformar a integral para os lados do elemento efetua-se a integral em $\theta$ analiticamente. Para isso é preciso definir explicitamente o raio em função do ângulo $\theta$, como mostra a figura 6.17.

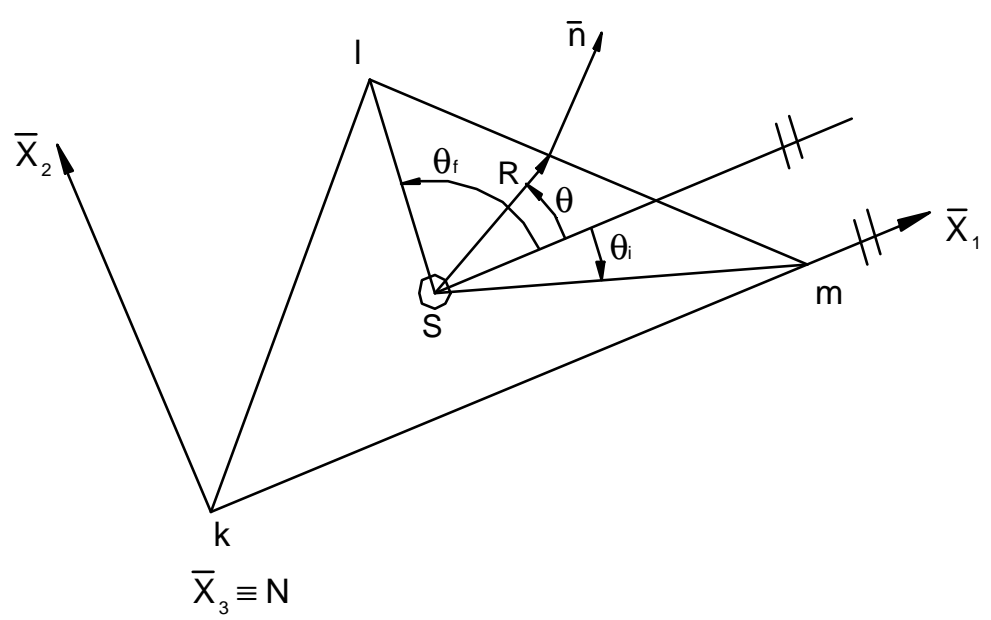

Figura 6.17 - Definição do raio em função do ângulo $\theta$

As coordenadas homogêneas de qualquer ponto do triângulo, como pode ser verificado em Coda (1990), são dadas pela seguinte expressão:

$$
\xi_{k}^{Q}=\xi_{k}^{S}+\frac{r}{2 \cdot A} \cdot\left(b^{k} \cdot \cos \theta+a^{k} \cdot \operatorname{sen} \theta\right)
$$

Onde: 


$$
\begin{aligned}
& a^{k}=\bar{X}_{1}^{l}-\bar{X}_{1}^{m} \\
& b^{k}=\bar{X}_{2}^{m}-\bar{X}_{2}^{l} \\
& A=\frac{1}{2} \cdot\left(b^{1} a^{2}-b^{2} a^{1}\right)
\end{aligned}
$$

Com:

$m=1,2,3 ; l=2,3,1$ e $k=3,1,2$.

Sendo assim, a distância do ponto $S$ a um ponto $Q$ pertencente ao lado $\mathrm{ml}$ é dado por:

$$
R_{m l}(S, Q)=\frac{-2 \cdot A \cdot \xi_{k}^{S}}{b^{k} \cdot \cos \theta+a^{k} \cdot \operatorname{sen} \theta}
$$

E as diferenciais do raio em relação æ̀ coordenadas globais são:

$$
\begin{aligned}
& r,_{1}=\frac{\partial r}{\partial \bar{X}_{1}} \cdot \frac{\partial \bar{X}_{1}}{\partial X_{1}}+\frac{\partial r}{\partial \bar{X}_{2}} \cdot \frac{\partial \bar{X}_{2}}{\partial X_{1}}+\frac{\partial r}{\partial \bar{X}_{3}} \cdot \frac{\partial \bar{X}_{3}}{\partial X_{1}}=\cos \theta \cdot \frac{\partial \bar{X}_{1}}{\partial X_{1}}+\operatorname{sen} \theta \cdot \frac{\partial \bar{X}_{2}}{\partial X_{1}} \\
& r,_{2}=\frac{\partial r}{\partial \bar{X}_{1}} \cdot \frac{\partial \bar{X}_{1}}{\partial X_{2}}+\frac{\partial r}{\partial \bar{X}_{2}} \cdot \frac{\partial \bar{X}_{2}}{\partial X_{2}}+\frac{\partial r}{\partial \bar{X}_{3}} \cdot \frac{\partial \bar{X}_{3}}{\partial X_{2}}=\cos \theta \cdot \frac{\partial \bar{X}_{1}}{\partial X_{2}}+\operatorname{sen} \theta \cdot \frac{\partial \bar{X}_{2}}{\partial X_{2}} \\
& r,,_{3}=\frac{\partial r}{\partial \bar{X}_{1}} \cdot \frac{\partial \bar{X}_{1}}{\partial X_{3}}+\frac{\partial r}{\partial \bar{X}_{2}} \cdot \frac{\partial \bar{X}_{2}}{\partial X_{3}}+\frac{\partial r}{\partial \bar{X}_{3}} \cdot \frac{\partial \bar{X}_{3}}{\partial X_{3}}=\cos \theta \cdot \frac{\partial \bar{X}_{1}}{\partial X_{3}}+\operatorname{sen} \theta \cdot \frac{\partial \bar{X}_{2}}{\partial X_{3}}
\end{aligned}
$$

Generalizando:

$$
r,_{i}=\cos \theta \cdot \frac{\partial \bar{X}_{1}}{\partial X_{i}}+\operatorname{sen} \theta \cdot \frac{\partial \bar{X}_{2}}{\partial X_{i}}
$$

Substituindo-se a solução fundamental de Kelvin na integral (6.44), tem-se:

$$
g_{i j}=\int_{\theta_{i}}^{\theta_{f}} \int_{0}^{R} \frac{1}{16 \cdot \pi \cdot G \cdot(1-v) \cdot r} \cdot\left[(3-4 \cdot v) \cdot \delta_{i j}+r,_{i} \cdot r,{ }_{j}\right] \cdot r \cdot d r \cdot d \theta
$$

Integrando-se em r, obtém-se:

$$
g_{i j}=\int_{\theta_{i}}^{\theta_{f}} \frac{1}{16 \cdot \pi \cdot G \cdot(1-v)} \cdot\left[(3-4 \cdot v) \cdot \delta_{i j}+r,{ }_{i} \cdot r, j\right] \cdot R \cdot d \theta
$$


Substituindo-se o raio em função do ângulo $\theta$ e as diferenciais do raio apresentadas na equação (6.53), obtém-se a seguinte integral em termos de componentes para um lado genérico $\mathrm{ml}$ :

$$
\begin{aligned}
& g_{i j}=\frac{-2 \cdot A \cdot \xi_{k}^{S}}{16 \cdot \pi \cdot G \cdot(1-v)} \cdot\left\{(3-4 \cdot v) \cdot \delta_{i j} \cdot \int_{\theta_{i}}^{\theta_{f}} \frac{1}{b^{k} \cdot \cos \theta+a^{k} \cdot \operatorname{sen} \theta} \cdot d \theta+\right. \\
& \frac{\partial \bar{X}_{1}}{\partial X_{i}} \cdot \frac{\partial \bar{X}_{1}}{\partial X_{j}} \cdot \int_{\theta_{i}}^{\theta_{f}} \frac{\cos ^{2} \theta}{b^{k} \cdot \cos \theta+a^{k} \cdot \operatorname{sen} \theta} \cdot d \theta+ \\
& \left(\frac{\partial \bar{X}_{1}}{\partial X_{i}} \cdot \frac{\partial \bar{X}_{2}}{\partial X_{j}}+\frac{\partial \bar{X}_{1}}{\partial X_{j}} \cdot \frac{\partial \bar{X}_{2}}{\partial X_{i}}\right) \cdot \int_{\theta_{i}}^{\theta_{f}} \frac{\operatorname{sen} \theta \cdot \cos \theta}{b^{k} \cdot \cos \theta+a^{k} \cdot \operatorname{sen} \theta} \cdot d \theta+ \\
& \left.\frac{\partial \bar{X}_{2}}{\partial X_{i}} \cdot \frac{\partial \bar{X}_{2}}{\partial X_{j}} \cdot \int_{\theta_{i}}^{\theta_{f}} \frac{\operatorname{sen}^{2} \theta}{b^{k} \cdot \cos \theta+a^{k} \cdot \operatorname{sen} \theta} \cdot d \theta\right\}
\end{aligned}
$$

Onde:

$$
\begin{aligned}
& \xi_{k}^{S}=\frac{1}{3}, \text { para o elemento constante. } \\
& m=1,2,3 ; \mathrm{l}=2,3,1 \text { e } k=3,1,2 \\
& i, j=1,2,3
\end{aligned}
$$

Procedendo-se de forma análoga para a integral (6.45), tem-se:

$$
h_{i j}=\int_{\theta_{i}}^{\theta_{f}} \int_{0}^{R} \frac{1}{8 \cdot \pi \cdot(1-v) \cdot r^{2}} \cdot(1-2 \cdot v) \cdot\left(r,{ }_{i} \cdot \eta_{j}-r,{ }_{j} \cdot \eta_{i}\right) \cdot r \cdot d r \cdot d \theta
$$

Avaliando esta integral em termos de valor principal de Cauchy, como demonstrado no anexo A, obtém-se:

$$
h_{i j}=\int_{\theta_{i}}^{\theta_{f}} \frac{(1-2 \cdot v)}{8 \cdot \pi \cdot(1-v)} \cdot\left(r_{i} \cdot \eta_{j}-r,{ }_{j} \cdot \eta_{i}\right) \cdot \ln R \cdot d \theta
$$

Efetuando-se a substituição do raio em função de teta e suas diferenciais, tem-se em termos de componentes para um lado genérico $\mathrm{ml}$ : 


$$
\begin{aligned}
h_{i j}= & \frac{(1-2 \cdot v) \cdot\left(1-\delta_{i j}\right)}{8 \cdot \pi \cdot(1-v)} \cdot\left\{( \frac { \partial \overline { X } _ { 1 } } { \partial X _ { i } } \cdot \eta _ { j } - \frac { \partial \overline { X } _ { 1 } } { \partial X _ { j } } \cdot \eta _ { i } ) \cdot \left[\left.\ln \left(2 \cdot A \cdot \xi_{k}^{S}\right) \cdot \operatorname{sen} \theta\right|_{\theta_{i}} ^{\theta_{f}}-\right.\right. \\
& \left.\operatorname{sen} \theta \cdot \ln \left[-\left(b^{k} \cdot \cos \theta+a^{k} \cdot \operatorname{sen} \theta\right)\right]\right|_{\theta_{i}} ^{\theta_{f}}+a^{k} \cdot \int_{\theta_{i}}^{\theta_{i}} \frac{\operatorname{sen} \theta \cdot \cos \theta}{b^{k} \cdot \cos \theta+a^{k} \cdot \operatorname{sen} \theta} \cdot d \theta- \\
& \left.b^{k} \cdot \int_{\theta_{i}}^{\theta_{f}} \frac{\operatorname{sen}^{2} \theta}{b^{k} \cdot \cos \theta+a^{k} \cdot \operatorname{sen} \theta} \cdot d \theta\right]+\left(\frac{\partial \bar{X}_{2}}{\partial X_{i}} \cdot \eta_{j}-\frac{\partial \bar{X}_{2}}{\partial X_{j}} \cdot \eta_{i}\right) \cdot[ \\
-\left.\ln \left(2 \cdot A \cdot \xi_{k}^{S}\right) \cdot \cos \theta\right|_{\theta_{i}} ^{\theta_{f}}+\left.\cos \theta \cdot \ln \left[-\left(b^{k} \cdot \cos \theta+a^{k} \cdot \operatorname{sen} \theta\right)\right]\right|_{\theta_{i}} ^{\theta_{f}}+ & \left.\left.b^{k} \cdot \int_{\theta_{i}}^{\theta_{f}} \frac{\operatorname{sen} \theta \cdot \cos \theta}{b^{k} \cdot \cos \theta+a^{k} \cdot \operatorname{sen} \theta} \cdot d \theta-a^{k} \cdot \int_{\theta_{i}}^{\theta_{f}} \frac{\cos ^{2} \theta}{b^{k} \cdot \cos \theta+a^{k} \cdot \operatorname{sen} \theta} \cdot d \theta\right]\right\}
\end{aligned}
$$

Com:

$m=1,2,3 ; l=2,3,1$ e $k=3,1,2$.

$\mathrm{i}, \mathrm{j}=1,2,3$.

As primitivas das integrais com a variável em $\theta$ estão mostradas no anexo.

Para o elemento linear, a integral singular foi calculada apenas semianaliticamente, seguindo o primeiro procedimento, efetuando-se a integração analítica em $r$ e numericamente em teta com a quadratura de Gauss, sem transformar para uma integral linear sobre os lados, como pode ser visto na figura 6.18.

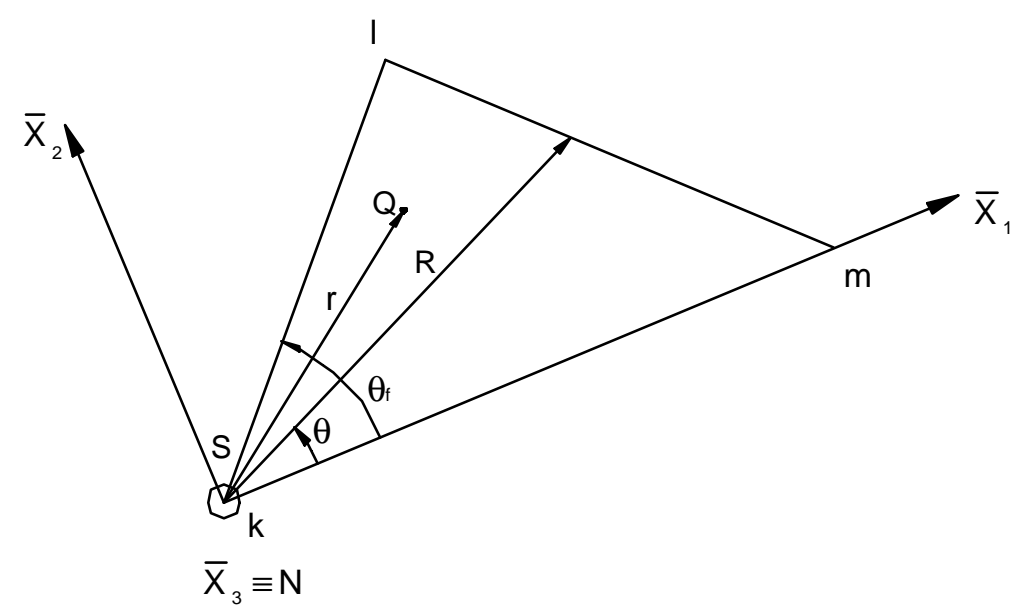

Figura 6.18 - Integral semi-analítica sobre o elemento linear 
As coordenadas homogêneas de um ponto qualquer do triângulo são obtidas pela expressão (6.50) e o raio pela expressão (6.52). Para o elemento linear, as coordenadas homogêneas do ponto $S$ assumem os seguintes valores:

$$
p /\left\{\begin{array}{l}
S=k \Rightarrow \xi_{k}^{S}=1 \\
S \neq k \Rightarrow \xi_{k}^{S}=0
\end{array}\right.
$$

Com isso a integral (6.44) fica:

$$
g_{i j}=\int_{\theta_{i}}^{\theta_{f}} \int_{0}^{R} \frac{1}{16 \cdot \pi \cdot G \cdot(1-v) \cdot r} \cdot\left[(3-4 \cdot v) \cdot \delta_{i j}+r,{ }_{i} \cdot r,{ }_{j}\right] \cdot \xi_{k}^{Q} \cdot r \cdot d r \cdot d \theta
$$

Substituindo-se a expressão de coordenada homogênea genérica de um ponto qualquer do triângulo, tem-se:

$$
\begin{gathered}
g_{i j}=\int_{\theta_{i}}^{\theta_{f}} \int_{0}^{R} \frac{1}{16 \cdot \pi \cdot G \cdot(1-v)} \cdot\left[(3-4 \cdot v) \cdot \delta_{i j}+r,{ }_{i} \cdot r,{ }_{j}\right] \\
\\
{\left[\xi_{k}^{S}+\frac{r}{2 \cdot A} \cdot\left(b^{k} \cdot \cos \theta+a^{k} \cdot \operatorname{sen} \theta\right)\right] \cdot d r \cdot d \theta}
\end{gathered}
$$

Efetuando-se a integral em r:

$$
\begin{gathered}
g_{i j}=\int_{\theta_{i}}^{\theta_{f}} \frac{1}{16 \cdot \pi \cdot G \cdot(1-v) \cdot r} \cdot\left[(3-4 \cdot v) \cdot \delta_{i j}+r,{ }_{i} \cdot r,{ }_{j}\right] . \\
\\
{\left[\xi_{k}^{S} \cdot R+\frac{R^{2}}{4 \cdot A} \cdot\left(b^{k} \cdot \cos \theta+a^{k} \cdot \operatorname{sen} \theta\right)\right] \cdot d \theta}
\end{gathered}
$$

Aplicando-se a quadratura de Gauss, obtém-se o seguinte somatório:

$$
\begin{aligned}
& g_{i j}= \frac{\theta_{f}-\theta_{i}}{2} \cdot \sum_{i g=1}^{i g \theta} \frac{1}{16 \cdot \pi \cdot G \cdot(1-v) \cdot r} \cdot\left[(3-4 \cdot v) \cdot \delta_{i j}+r,,_{i} \cdot r, j\right. \\
& \\
& {\left[\xi_{k}^{S} \cdot R+\frac{R^{2}}{4 \cdot A} \cdot\left(b^{k} \cdot \cos \theta+a^{k} \cdot \operatorname{sen} \theta\right)\right] }
\end{aligned}
$$

Para a integral (6.45) procede-se de forma análoga:

$$
h_{i j}=\int_{\theta_{i}}^{\theta_{f}} \int_{0}^{R} \frac{1}{8 \cdot \pi \cdot(1-v) \cdot r^{2}} \cdot(1-2 \cdot v) \cdot\left(r,{ }_{i} \cdot \eta_{j}-r,{ }_{j} \cdot \eta_{i}\right) \cdot \xi_{k}^{Q} \cdot r \cdot d r \cdot d \theta
$$


Substituindo-se a expressão de coordenada homogênea genérica:

$$
\begin{aligned}
h_{i j}= & \int_{\theta_{i}}^{\theta_{f}} \int_{0}^{R} \frac{1}{8 \cdot \pi \cdot(1-v)} \cdot(1-2 \cdot v) \cdot\left(r,{ }_{i} \cdot \eta_{j}-r,{ }_{j} \cdot \eta_{i}\right) . \\
& {\left[\frac{\xi_{k}^{S}}{r}+\frac{1}{2 \cdot A} \cdot\left(b^{k} \cdot \cos \theta+a^{k} \cdot \operatorname{sen} \theta\right)\right] \cdot d r \cdot d \theta }
\end{aligned}
$$

Lembra-se que esta integral á avaliada como valor principal de Cauchy, conforme demonstrado no anexo $\mathrm{A}$, tem-se:

$$
\begin{aligned}
h_{i j}= & \int_{\theta_{i}}^{\theta_{f}} \frac{(1-2 \cdot v)}{8 \cdot \pi \cdot(1-v)} \cdot\left(r,{ }_{i} \cdot \eta_{j}-r,{ }_{j} \cdot \eta_{i}\right) . \\
& {\left[\xi_{k}^{S} \cdot \ln R+\frac{R}{2 \cdot A} \cdot\left(b^{k} \cdot \cos \theta+a^{k} \cdot \operatorname{sen} \theta\right)\right] \cdot d \theta }
\end{aligned}
$$

Aplicando-se a quadratura de Gauss, obtém-se o somatório:

$$
\begin{array}{r}
h_{i j}=\frac{\theta_{f}-\theta_{i}}{2} \cdot \sum_{i g=1}^{i g \theta} \frac{(1-2 \cdot v)}{8 \cdot \pi \cdot(1-v)} \cdot\left(r,,_{i} \cdot \eta_{j}-r,,_{j} \cdot \eta_{i}\right) . \\
{\left[\xi_{k}^{S} \cdot \ln R+\frac{R}{2 \cdot A} \cdot\left(b^{k} \cdot \cos \theta+a^{k} \cdot \operatorname{sen} \theta\right)\right]}
\end{array}
$$

Para efetuar esses somatórios, os ângulos $\theta_{\mathrm{f}}$ e $\theta_{\mathrm{i}}$ devem estar em radianos.

Para o elemento quadrático a integral singular é obtida de forma análoga ao demonstrado para o elemento linear, bastando utilizar agora as funções de forma do elemento quadrático apresentadas em (6.19).

No caso em que as funções de forma são $\Phi_{1}, \Phi_{2}$ ou $\Phi_{3}$, tem-se:

$$
\Phi_{p}^{Q}=\xi_{k}^{Q} \cdot\left(2 \cdot \xi_{k}^{Q}-1\right)=2 \cdot\left(\xi_{k}^{Q}\right)^{2}-\xi_{k}^{Q}, \quad \mathrm{p}=\mathrm{k}=1,2,3
$$

Substituindo-se as coordenadas homogêneas do ponto $Q$ em função do ponto S, já mostrado em (6.50), resulta:

$$
\Phi_{p}^{Q}=2 \cdot\left[\xi_{k}^{S}+\frac{r}{2 \cdot A} \cdot\left(b^{k} \cdot \cos \theta+a^{k} \cdot \operatorname{sen} \theta\right)\right]^{2}-\left[\xi_{k}^{S}+\frac{r}{2 \cdot A} \cdot\left(b^{k} \cdot \cos \theta+a^{k} \cdot \operatorname{sen} \theta\right)\right]
$$

Desenvolvendo esta expressão, tem-se: 


$$
\begin{aligned}
\Phi_{p}^{Q}= & 2 \cdot\left(\xi_{k}^{S}\right)^{2}-\xi_{k}^{S}+\frac{r}{2 \cdot A} \cdot\left(b^{k} \cdot \cos \theta+a^{k} \cdot \operatorname{sen} \theta\right) \cdot\left(4 \cdot \xi_{k}^{S}-1\right) \\
& +\frac{r^{2}}{2 \cdot A^{2}} \cdot\left(b^{k} \cdot \cos \theta+a^{k} \cdot \operatorname{sen} \theta\right)^{2}
\end{aligned}
$$

E para o caso em que as funções de forma são $\Phi_{4}, \Phi_{5}$ ou $\Phi_{6}$, tem-se:

$$
\Phi_{p}^{Q}=4 \cdot \xi_{k}^{Q} \cdot \xi_{m}^{Q}, \quad \mathrm{p}=4,5,6 ; \mathrm{k}=1,2,3 \text { e } \mathrm{m}=2,3,1
$$

Substituindo-se as coordenadas homogêneas em função das coordenadas do ponto fonte $\mathrm{S}$ e desenvolvendo a expressão, obtém-se:

$$
\begin{aligned}
\Phi_{p}^{Q} & =4 \cdot\left\{\xi_{k}^{S} \cdot \xi_{m}^{S}+\frac{r}{2 \cdot A} \cdot\left[\xi_{k}^{S} \cdot\left(b^{m} \cdot \cos \theta+a^{m} \cdot \operatorname{sen} \theta\right)+\xi_{m}^{S} \cdot\left(b^{k} \cdot \cos \theta+a^{k} \cdot \operatorname{sen} \theta\right)\right]\right. \\
& \left.+\frac{r^{2}}{4 \cdot A^{2}} \cdot\left(b^{k} \cdot \cos \theta+a^{k} \cdot \operatorname{sen} \theta\right) \cdot\left(b^{m} \cdot \cos \theta+a^{m} \cdot \operatorname{sen} \theta\right)\right\}
\end{aligned}
$$

Substituindo essas funções de forma na integral (6.44) e (6.45) e desenvolvendo como no elemento linear, obtém-se as integrais singulares para 0 elemento quadrático. Para as funções de forma $\Phi_{1}, \Phi_{2}$ ou $\Phi_{3}$ :

$$
\begin{aligned}
g_{i j}= & \frac{\theta_{f}-\theta_{i}}{2} \cdot \sum_{i g=1}^{i g \theta} \frac{1}{16 \cdot \pi \cdot G \cdot(1-v)} \cdot\left[(3-4 \cdot v) \cdot \delta_{i j}+r,,_{i} \cdot r, j\right] \\
& \left\{R \cdot\left[2 \cdot\left(\xi_{k}^{S}\right)^{2}-\xi_{k}^{S}\right]+\frac{R^{2}}{4 \cdot A} \cdot\left(b^{k} \cdot \cos \theta+a^{k} \cdot \operatorname{sen} \theta\right) \cdot\left(4 \cdot \xi_{k}^{S}-1\right)\right. \\
& \left.+\frac{R^{3}}{6 \cdot A^{2}} \cdot\left(b^{k} \cdot \cos \theta+a^{k} \cdot \operatorname{sen} \theta\right)^{2}\right\} \cdot w_{i g} \\
h_{i j}= & \frac{\theta_{f}-\theta_{i}}{2} \cdot \sum_{i g=1}^{i g \theta} \frac{(1-2 \cdot v)}{8 \cdot \pi \cdot(1-v)} \cdot\left(r,,_{i} \cdot \eta_{j}-r,,_{j} \cdot \eta_{i}\right) \cdot \\
& \left\{\left[2 \cdot\left(\xi_{k}^{S}\right)^{2}-\xi_{k}^{S}\right] \cdot \ln R+\frac{R}{2 \cdot A} \cdot\left(b^{k} \cdot \cos \theta+a^{k} \cdot \operatorname{sen} \theta\right) \cdot\left(4 \cdot \xi_{k}^{S}-1\right)\right. \\
& \left.+\frac{R^{2}}{4 \cdot A^{2}} \cdot\left(b^{k} \cdot \cos \theta+a^{k} \cdot \operatorname{sen} \theta\right)^{2}\right\} \cdot w_{i g}
\end{aligned}
$$

Para as funções de forma $\Phi_{4}, \Phi_{5}$ ou $\Phi_{6}$, essas integrais são: 


$$
\begin{aligned}
g_{i j}= & \frac{\theta_{f}-\theta_{i}}{2} \cdot \sum_{i g=1}^{i g \theta} \frac{1}{16 \cdot \pi \cdot G \cdot(1-v)} \cdot\left[(3-4 \cdot v) \cdot \delta_{i j}+r,{ }_{i} \cdot r,{ }_{j}\right] \cdot\left\{4 \cdot \xi_{k}^{S} \cdot \xi_{m}^{S} \cdot R\right. \\
& +\frac{R^{2}}{A} \cdot\left[\xi_{k}^{S} \cdot\left(b^{m} \cdot \cos \theta+a^{m} \cdot \operatorname{sen} \theta\right)+\xi_{m}^{S} \cdot\left(b^{k} \cdot \cos \theta+a^{k} \cdot \operatorname{sen} \theta\right)\right] \\
& \left.+\frac{R^{3}}{3 \cdot A^{2}} \cdot\left(b^{k} \cdot \cos \theta+a^{k} \cdot \operatorname{sen} \theta\right) \cdot\left(b^{m} \cdot \cos \theta+a^{m} \cdot \operatorname{sen} \theta\right)\right\} \cdot w_{i g} \\
h_{i j}= & \frac{\theta_{f}-\theta_{i}}{2} \cdot \sum_{i g=1}^{i g \theta} \frac{(1-2 \cdot v)}{8 \cdot \pi \cdot(1-v)} \cdot\left(r,{ }_{i} \cdot \eta_{j}-r,,_{j} \cdot \eta_{i}\right) \cdot\left\{4 \cdot \xi_{k}^{S} \cdot \xi_{m}^{S} \cdot \ln R\right. \\
& +\frac{2 \cdot R}{A} \cdot\left[\xi_{k}^{S} \cdot\left(b^{m} \cdot \cos \theta+a^{m} \cdot \operatorname{sen} \theta\right)+\xi_{m}^{S} \cdot\left(b^{k} \cdot \cos \theta+a^{k} \cdot \operatorname{sen} \theta\right)\right] \\
& \left.+\frac{R^{2}}{2 \cdot A^{2}} \cdot\left(b^{k} \cdot \cos \theta+a^{k} \cdot \operatorname{sen} \theta\right) \cdot\left(b^{m} \cdot \cos \theta+a^{m} \cdot \operatorname{sen} \theta\right)\right\} \cdot w_{i g}
\end{aligned}
$$

No caso em que o ponto fonte coincide geometricamente com os vértices do triângulo (nós 1, 2 e 3), as coordenadas homogêneas do ponto $S$ assumem valores iguais a 1 ou 0 . E para o caso em que o ponto fonte não coincide geometricamente com os nós das faces do triângulo (nós 4, 5 e 6), calculam-se as coordenadas homogêneas do ponto $S$, lembrando que nesse caso é preciso dividir o elemento em dois triângulos, como feito na integral quase singular e mostrado na figura 6.14.

Para o cálculo dos termos livres utiliza-se a propriedade de movimento de corpo rígido tanto para o elemento linear quanto para o elemento quadrático.

\subsection{Montagem do sistema de equações}

Para montar o sistema de equações torna-se necessário calcular as componentes das matrizes $\mathrm{H}$ e $\mathrm{G}$, e para isso toma-se como ponto fonte todos os nós dos elementos discretizados e todos os pontos internos e integram-se todos os elementos do contorno. Neste trabalho optou-se por calcular os deslocamentos em pontos internos no sistema linear principal, por esta técnica conferir uma maior precisão aos resultados, apesar de aumentar o número de graus de liberdade das matrizes. A seguir é mostrado um fluxograma, com os principais passos do programa computacional no que diz respeito àgeração do sistema de equações. 
Sim

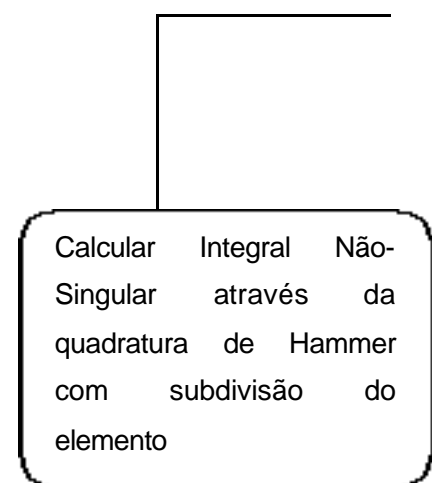

Entrada de Dados:

Leitura, Propriedades,

Local do Ponto Fonte,

Alocação de Memória

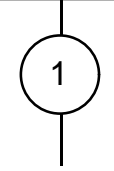

Ponto Fonte está

longe do Elemento

a ser Integrado ou

é Ponto Interno?
Calcular integral Singular

Semi-Analiticamente

Analiticamente

Constante) ou

(Elemento

rar

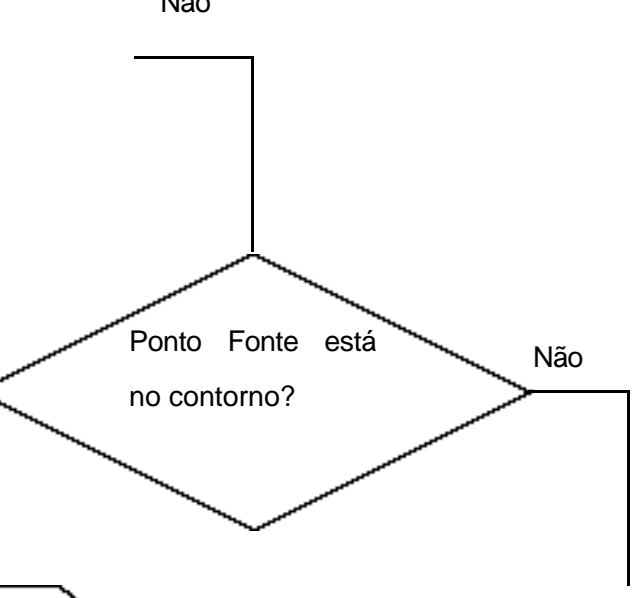

Calcular integral Quase

Singular através da

Quadratura de Gauss

Alocação dos Termos Livres na Matriz $\mathrm{H}$,

Troca das Colunas das Matrizes e

Resolução do Sistema Linear

Figura 6.19 - Fluxograma da montagem das equações 


\subsection{Deslocamentos e tensões em pontos internos}

Depois de calculados todos os valores de deslocamentos do contorno e forças de superfície, é necessário calcular os valores de deslocamentos e tensões em pontos internos. Para obter os deslocamentos nos pontos internos utiliza-se a equação integral (5.22), chegando-se a uma equação semelhante à(6.6a):

$$
\begin{aligned}
& \underset{\sim}{u}(s)=-\sum_{i=1}^{L}\left[\int_{\Gamma_{i}} p^{*}(s, Q) \cdot \underset{\sim}{\Phi^{T}}(Q) \cdot d \Gamma(Q)\right] \cdot{\underset{\sim}{U}}^{N}(Q) \\
& +\sum_{i=1}^{L}\left[\int_{\Gamma_{i}} u^{*}(s, Q) \cdot{\underset{\sim}{\sim}}^{T}(Q) \cdot d \Gamma(Q)\right] \cdot P_{\sim}^{N}(Q) \\
& +\sum_{j=1}^{M}\left[{\underset{\Omega_{j}}{\underset{\sim}{u}}}_{u^{*}}(s, q) \cdot{\underset{\sim}{\Phi_{c}}}^{T}(q) \cdot d \Omega(q)\right] \cdot \boldsymbol{\sim}^{N}(q)
\end{aligned}
$$

Que sob forma matricial assume o seguinte aspecto:

$$
\underset{\sim}{u}=-\underset{\sim}{H} \cdot \underset{\sim}{U}+\underset{\sim}{G} \cdot \underset{\sim}{P}+\underset{\sim}{D} B
$$

Onde:

$$
\underset{\sim}{u}(s)=\left\{\begin{array}{l}
u_{1} \\
u_{2} \\
u_{3}
\end{array}\right\}
$$

Utiliza-se o procedimento de integração não singular, já discutido anteriormente para pontos no contorno. Vale lembrar que neste trabalho optou-se por calcular os deslocamentos internos através do sistema linear principal, junto com os valores de contorno, e não depois de calculados os valores de contorno, como fora apresentado. Isto levou a uma maior precisão nos resultados obtidos, mas apresenta o inconveniente de aumentar o número de graus de liberdade das matrizes. Para efetuar o cálculo dos deslocamentos internos desta forma, calculase as componentes das matrizes $\mathrm{H}$ e $\mathrm{G}$ normalmente e depois se acrescentam os termos livres referentes a pontos internos, que é igual a 1, na diagonal correspondente ao grau de liberdade do ponto interno, como mostrado em (6.82). 


$$
\begin{gathered}
\vdots \\
3 i-2 \\
3 i-1 \\
3 i \\
\vdots \\
\vdots
\end{gathered}\left[\begin{array}{llllll}
\ldots & 3 i-2 & 3 i-1 & 3 i & \ldots \\
& \vdots & \vdots & \vdots & \\
\cdots & 1 & 0 & 0 & \ldots \\
\cdots & 0 & 1 & 0 & \ldots \\
& \vdots & \vdots & \vdots &
\end{array}\right]
$$

Para se obter o tensor de tensões internas, utiliza-se a equação (5.23), obtendo-se uma equação semelhante à equação para deslocamentos nos pontos internos:

$$
\begin{aligned}
& \underset{\sim}{\sigma}(s)=-\sum_{i=1}^{L}\left[\int_{\Gamma_{i}} \underset{\sim}{S^{*}}(s, Q) \cdot{\underset{\sim}{\Phi}}^{T}(Q) \cdot d \Gamma(Q)\right] \cdot \underset{\sim}{U^{N}}(Q) \\
& +\sum_{i=1}^{L}\left[\int_{\Gamma_{i}} D^{*}(s, Q) \cdot \Phi_{\sim}^{T}(Q) \cdot d \Gamma(Q)\right] \cdot P_{\sim}^{N}(Q) \\
& +\sum_{j=1}^{M}\left[\int_{\Omega_{j}}{\underset{\sim}{*}}^{*}(s, q) \cdot{\underset{\sim}{\Phi}}^{T}(q) \cdot d \Omega(q)\right] \cdot{\underset{\sim}{B}}^{N}(q)
\end{aligned}
$$

Que matricialmente é representada por:

$$
\underset{\sim}{\sigma}=-\underset{\sim}{H} \cdot \underset{\sim}{U}+\underset{\sim}{\bar{G}} \cdot \underset{\sim}{P}+\underset{\sim}{\bar{D}} \cdot \underset{\sim}{B}
$$

Onde a utilização do traço serve apenas para diferenciar as matrizes envolvidas daquelas utilizadas na equação (6.80). E os tensores $D^{*}$ e $S^{*}$ estão definidos nas equações (5.24) e (5.25) respectivamente.

O tensor de tensões internas é dado pelos seguintes valores:

$$
\underset{\sim}{\sigma}(s)=\left[\begin{array}{lll}
\sigma_{11} & \sigma_{12} & \sigma_{13} \\
\sigma_{21} & \sigma_{22} & \sigma_{23} \\
\sigma_{31} & \sigma_{32} & \sigma_{33}
\end{array}\right]
$$

\subsection{Tensões em pontos do contorno}

Para analisar as tensões em pontos do contorno utilizar-se-á o procedimento indicado por Brebbia et al. (1984), o qual consiste em utilizar uma aproximação para 
as deformações a partir de valores de deslocamentos dos nós dos elementos. A escolha deste procedimento se deve ao fato de não ser necessário executar o limite da equação (5.23) quando o ponto fonte tende a ir para o contorno, o que segundo Brebbia et al. (1984) não produz valores precisos. Nenhuma integração adicional é necessária, o que gera uma economia no tempo de processamento.

Seja um ponto nodal como o apresentado na figura 6.20; as componentes de tensão podem ser representadas através de um elemento infinitesimal, onde as componentes de tensão na direção 3 são as próprias forças de superfície neste nó.

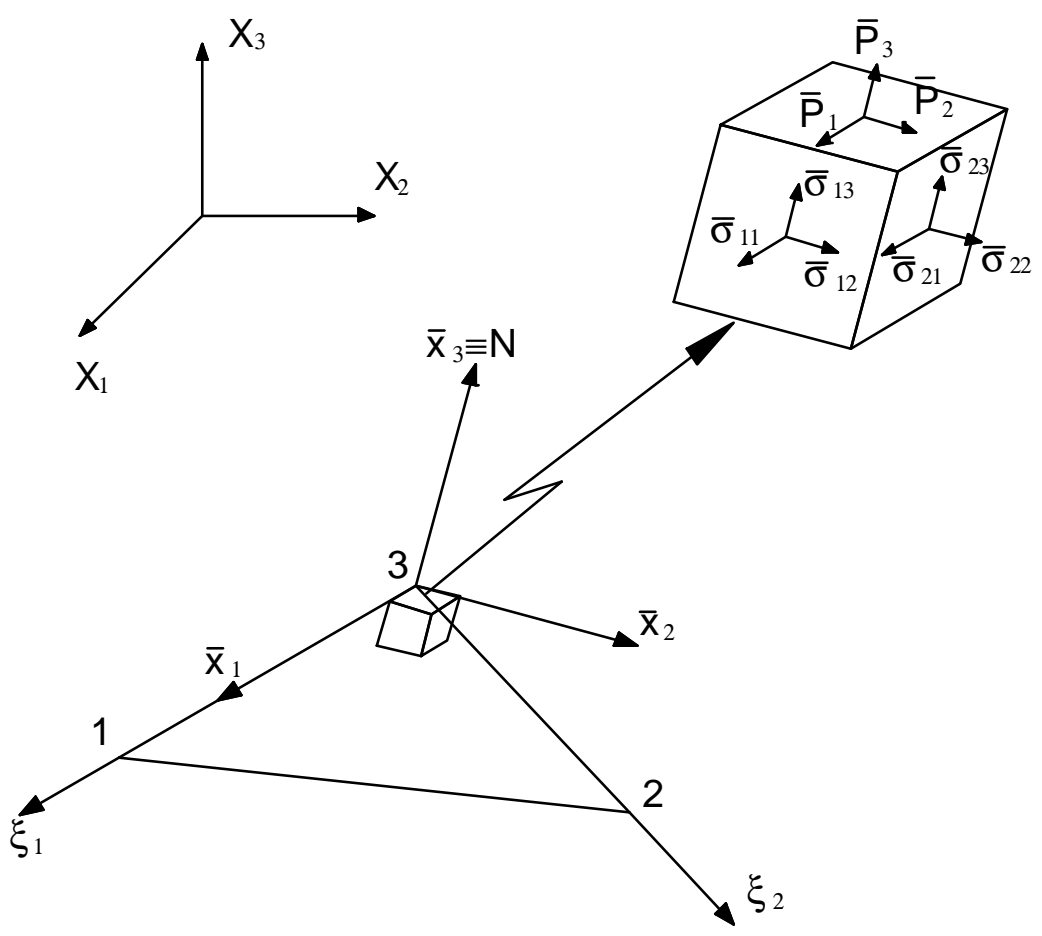

Figura 6.20 - Análise das tensões em ponto do contorno

As componentes de deslocamento em coordenadas locais são dadas por:

$$
\bar{\sim}=\underset{\sim}{\Phi^{T}} \cdot \bar{\sim}
$$

Onde:

$\bar{U}$ : deslocamentos nodais do elemento nas coordenadas locais

$\Phi^{T}:$ função aproximadora 
Que em termos de componentes:

$$
\bar{u}_{i}\left(\xi_{1}, \xi_{2}\right)=\xi_{1} \cdot\left(\bar{U}_{i}^{1}-\bar{U}_{i}^{3}\right)+\xi_{2} \cdot\left(\bar{U}_{i}^{2}-\bar{U}_{i}^{3}\right)+\bar{U}_{i}^{3}
$$

E possui derivadas em relação às coordenadas homogêneas:

$$
\begin{aligned}
& \frac{\partial \bar{u}_{i}}{\partial \xi_{1}}=\bar{U}_{i}^{1}-\bar{U}_{i}^{3} \\
& \frac{\partial \bar{u}_{i}}{\partial \xi_{2}}=\bar{U}_{i}^{2}-\bar{U}_{i}^{3}
\end{aligned}
$$

Onde os deslocamentos nodais em coordenadas locais podem ser obtidos através da rotação dos deslocamentos em coordenadas globais:

$$
\bar{U}=T_{\sim}^{T} \cdot \underset{\sim}{U}
$$

Sendo $T$ a matriz de transformação entre os eixos global e local, e cujas componentes são os co-senos diretores mostrados na figura 6.21:

$$
\underset{\sim}{T}=\left[\begin{array}{ccc}
l_{1} & l_{2} & l_{3} \\
m_{1} & m_{2} & m_{3} \\
n_{1} & n_{2} & n_{3}
\end{array}\right]
$$

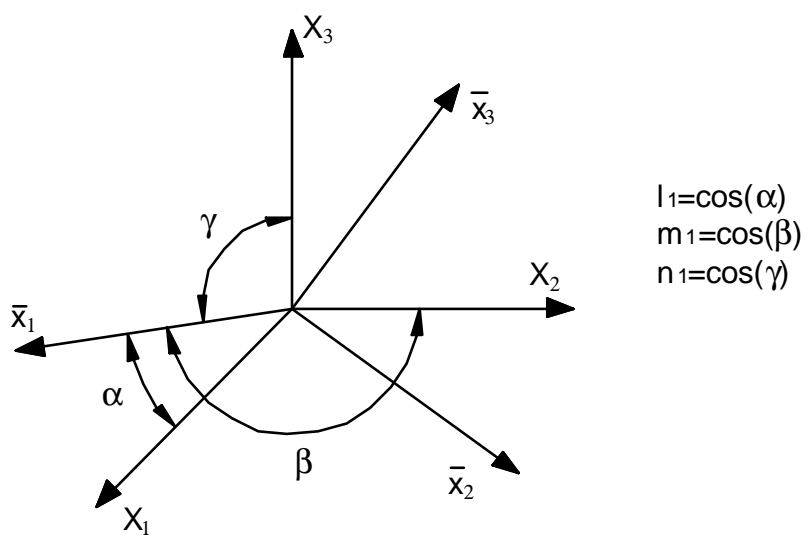

Figura 6.21 - Co-senos diretores

De modo análogo, as coordenadas cartesianas também são escritas como os deslocamentos: 


$$
\bar{x}_{i}\left(\xi_{1}, \xi_{2}\right)=\xi_{1} \cdot\left(\bar{x}_{i}-\bar{x}_{i}^{3}\right)+\xi_{2} \cdot\left(\bar{x}_{i}^{2}-\bar{x}_{i}^{3}\right)+\bar{x}_{i}^{3}
$$

E suas derivadas em relação æ̀s coordenadas homogêneas são:

$$
\begin{aligned}
& \frac{\partial \bar{x}_{i}}{\partial \xi_{1}}=\bar{x}_{i}-\bar{x}_{i}^{3} \\
& \frac{\partial \bar{x}_{i}}{\partial \xi_{2}}=\bar{x}_{i}^{2}-\bar{x}_{i}^{3}
\end{aligned}
$$

Da mesma forma que para deslocamentos, as coordenadas locais podem ser obtidas pela rotação de eixos:

$$
\bar{x}=T^{T} \cdot x
$$

As componentes de deformações sobre a superfície do elemento em coordenadas locais são dadas por:

$$
\bar{\varepsilon}_{i j}=\frac{1}{2} \cdot\left(\frac{\partial \bar{u}_{j}}{\partial \bar{x}_{i}}+\frac{\partial \bar{u}_{i}}{\partial \bar{x}_{j}}\right) \quad \text { com } i, j=1,2
$$

Pela equação (6.10) que mostra a relação de diferenciais de uma função em relação ao sistema de coordenadas cartesianas e as diferenciais desta função em relação æ̀s coordenadas homogêneas, tem-se para o plano do elemento:

$$
\left\{\begin{array}{l}
\frac{\partial \bar{u}_{i}}{\partial \xi_{1}} \\
\frac{\partial \bar{u}_{i}}{\partial \xi_{2}}
\end{array}\right\}=\left[\begin{array}{ll}
\frac{\partial \bar{x}_{1}}{\partial \xi_{1}} & \frac{\partial \bar{x}_{2}}{\partial \xi_{1}} \\
\frac{\partial \bar{x}_{1}}{\partial \xi_{2}} & \frac{\partial \bar{x}_{2}}{\partial \xi_{2}}
\end{array}\right] \cdot\left\{\begin{array}{l}
\frac{\partial \bar{u}_{i}}{\partial \bar{x}_{1}} \\
\frac{\partial \bar{u}_{i}}{\partial \bar{x}_{2}}
\end{array}\right\}=J \cdot\left\{\begin{array}{l}
\frac{\partial \bar{u}_{i}}{\partial \bar{x}_{1}} \\
\frac{\partial \bar{u}_{i}}{\partial \bar{x}_{2}}
\end{array}\right\}
$$

Onde o Jacobiano de transformação é $|G|=2 \cdot A$, apresentado em (6.13). Para se determinar as deformações, basta obter o inverso do Jacobiano, ou seja, a matriz inversa das diferenciais entre os sistemas de coordenadas, pois todos os outros parâmetros já foram definidos:

$$
\left\{\begin{array}{l}
\frac{\partial \bar{u}_{i}}{\partial \bar{x}_{1}} \\
\frac{\partial \bar{u}_{i}}{\partial \bar{x}_{2}}
\end{array}\right\}=\frac{1}{2 \cdot A} \cdot\left[\begin{array}{cc}
\frac{\partial \bar{x}_{2}}{\partial \xi_{2}} & -\frac{\partial \bar{x}_{2}}{\partial \xi_{1}} \\
-\frac{\partial \bar{x}_{1}}{\partial \xi_{2}} & \frac{\partial \bar{x}_{1}}{\partial \xi_{1}}
\end{array}\right] \cdot\left\{\begin{array}{l}
\frac{\partial \bar{u}_{i}}{\partial \xi_{1}} \\
\frac{\partial \bar{u}_{i}}{\partial \xi_{2}}
\end{array}\right\}
$$


Através da Lei de Hooke (4.10) e aplicando-se a fórmula de Cauchy no ponto mostrado na figura 6.20, resultam as componentes de tensão em coordenadas locais:

$$
\begin{aligned}
& \bar{\sigma}_{11}=\frac{2 \cdot G \cdot v}{1-2 \cdot v} \cdot \bar{\varepsilon}_{11}+\frac{2 \cdot G \cdot v}{1-2 \cdot v} \cdot \bar{\varepsilon}_{22}+\frac{2 \cdot G \cdot v}{1-2 \cdot v} \cdot \bar{\varepsilon}_{33}+2 \cdot G \cdot \bar{\varepsilon}_{11} \\
& \bar{\sigma}_{12}=2 \cdot G \cdot \bar{\varepsilon}_{12} \\
& \bar{\sigma}_{22}=\frac{2 \cdot G \cdot v}{1-2 \cdot v} \cdot \bar{\varepsilon}_{11}+\frac{2 \cdot G \cdot v}{1-2 \cdot v} \cdot \bar{\varepsilon}_{22}+\frac{2 \cdot G \cdot v}{1-2 \cdot v} \cdot \bar{\varepsilon}_{33}+2 \cdot G \cdot \bar{\varepsilon}_{22} \\
& \bar{\sigma}_{31}=\bar{P}_{1} \\
& \bar{\sigma}_{32}=\bar{P}_{2} \\
& \bar{\sigma}_{33}=\bar{P}_{3}
\end{aligned}
$$

Para simplificar essa expressão define-se $\lambda$ como:

$$
\lambda=\frac{2 \cdot G \cdot v}{1-2 \cdot v}
$$

Substituindo-se na equação (6.96.a e 6.96.c), tem-se:

$$
\begin{aligned}
& \bar{\sigma}_{11}=(2 \cdot G+\lambda) \cdot \bar{\varepsilon}_{11}+\lambda \cdot\left(\bar{\varepsilon}_{22}+\bar{\varepsilon}_{33}\right) \\
& \bar{\sigma}_{12}=2 \cdot G \cdot \bar{\varepsilon}_{12} \\
& \bar{\sigma}_{22}=(2 \cdot G+\lambda) \cdot \bar{\varepsilon}_{22}+\lambda \cdot\left(\bar{\varepsilon}_{11}+\bar{\varepsilon}_{33}\right) \\
& \bar{\sigma}_{31}=\bar{P}_{1} \\
& \bar{\sigma}_{32}=\bar{P}_{2} \\
& \bar{\sigma}_{33}=\bar{P}_{3}
\end{aligned}
$$

Para a direção 3, tem-se que:

$$
\bar{\sigma}_{33}=\bar{P}_{3}=(2 \cdot G+\lambda) \cdot \bar{\varepsilon}_{33}+\lambda \cdot\left(\bar{\varepsilon}_{11}+\bar{\varepsilon}_{22}\right)
$$

Ou inversamente:

$$
\bar{\varepsilon}_{33}=\frac{1}{(2 \cdot G+\lambda)} \cdot\left[\bar{P}_{3}-\lambda \cdot\left(\bar{\varepsilon}_{11}+\bar{\varepsilon}_{22}\right)\right]
$$

Substituindo-se a equação (6.100) nas equações (6.98.a e 6.98.c), obtém-se as seguintes equações: 


$$
\begin{aligned}
& \bar{\sigma}_{11}=\frac{1}{1-v} \cdot\left[v \cdot \bar{P}_{3}+2 \cdot G \cdot\left(\bar{\varepsilon}_{11}+v \cdot \bar{\varepsilon}_{22}\right)\right] \\
& \bar{\sigma}_{12}=2 \cdot G \cdot \bar{\varepsilon}_{12} \\
& \bar{\sigma}_{22}=\frac{1}{1-v} \cdot\left[v \cdot \bar{P}_{3}+2 \cdot G \cdot\left(\bar{\varepsilon}_{22}+v \cdot \bar{\varepsilon}_{11}\right)\right] \\
& \bar{\sigma}_{31}=\bar{P}_{1} \\
& \bar{\sigma}_{32}=\bar{P}_{2} \\
& \bar{\sigma}_{33}=\bar{P}_{3}
\end{aligned}
$$

Onde as forças de superfície nas coordenadas locais podem ser obtidas através da rotação das forças de superfície nas coordenadas globais, já calculadas através da resolução do sistema de equações do MEC:

$$
\bar{P}=T^{T} \cdot P
$$

Tendo-se as componentes das tensões em coordenadas locais, basta rotacioná-las para as coordenadas globais:

$$
\underset{\sim}{\sigma}=\underset{\sim}{T} \cdot \underset{\sim}{\sigma} \cdot T_{\sim}^{T}
$$

Onde:

$T:$ matriz de transformação, já definida anteriormente. 


\section{CAPÍTULO 7}

\section{APLICAÇÕES NUMÉRICAS}

\subsection{Introdução}

Para analisar a formulação apresentada é preciso utilizá-la em exemplos numéricos com valores analíticos conhecidos e que já foram utilizados por outros pesquisadores da área para que se possa realizar uma comparação entre as diversas técnicas. Todos os exemplos numéricos calculados com elementos lineares e quadráticos utilizaram a técnica do ponto no contorno (integral singular semi-analítica) e a técnica do ponto fora, cujo fator de afastamento do ponto fonte será escrito em cada exemplo; para os exemplos calculados com elementos constantes utilizam-se a técnica do ponto no contorno (integral singular analítica e semi-analítica) e a técnica do ponto fora.

Adota-se a abreviatura CMEC para se referir ao método dos elementos de contorno clássico, o qual utiliza a solução fundamental de Kelvin; e a abreviatura HMEC quando se trata da utilização da solução hiper-singular em alguns pontos e a solução fundamental de Kelvin nos pontos restantes. Para a formulação do HMEC, baseada no trabalho de Ingber \& Rudolphi (1990), utiliza-se a solução fundamental de Kelvin em pontos que tenham forças de superfície prescritas e a solução hipersingular em pontos com deslocamentos prescritos. Deixa-se claro que a formulação HMEC só foi utilizada com o elemento linear. Ainda a respeito das abreviaturas, utiliza-se ISA para indicar a formulação na qual efetua-se a integral singular analiticamente, ISSA para a integral singular efetuada semi-analiticamente e ISQG 
para a integral quase singular calculada numericamente com quadratura de Gauss e subdivisão progressiva do raio.

\subsection{Exemplo 1 - Sólido tridimensional sob força axial}

Neste problema tem-se de um sólido elástico tridimensional, que possui base quadrangular vinculada, e é solicitado por uma força de tração distribuída na face superior. Este sólido possui propriedades geométricas e físicas apresentadas na figura 7.1 .

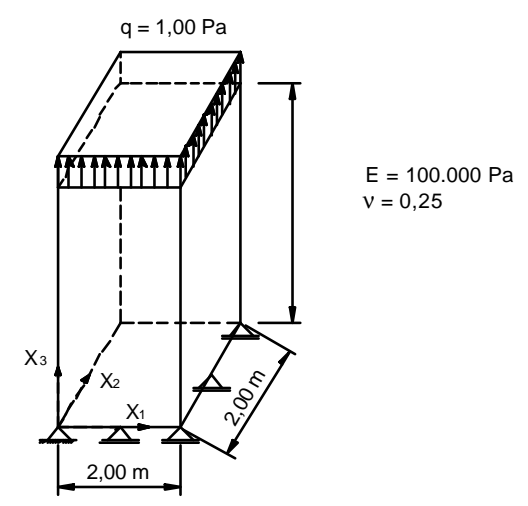

Figura 7.1 - Sólido tridimensional com força distribuída de tração e propriedades físicas e geométricas

Testou-se a formulação apresentada em uma discretização para os elementos linear e quadrático: 12 elementos (16 nós com 48 graus de liberdade (GL) para o elemento linear e 42 nós com 126 graus de liberdade para o elemento quadrático) e três discretizações para o elemento constante: 12 elementos (36 GL), 80 elementos (240 GL) e 180 elementos (540 GL), figura 7.2, com a finalidade de se ilustrar o processo de convergência do elemento constante.

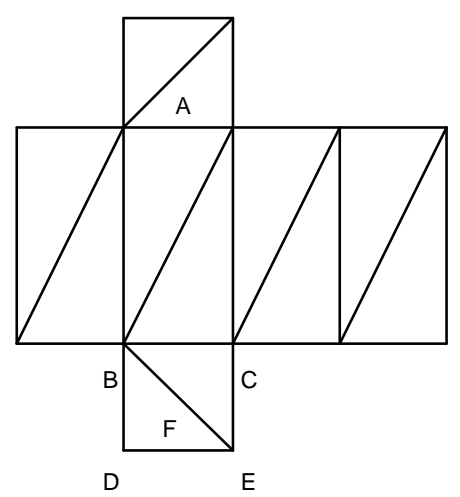

(a)

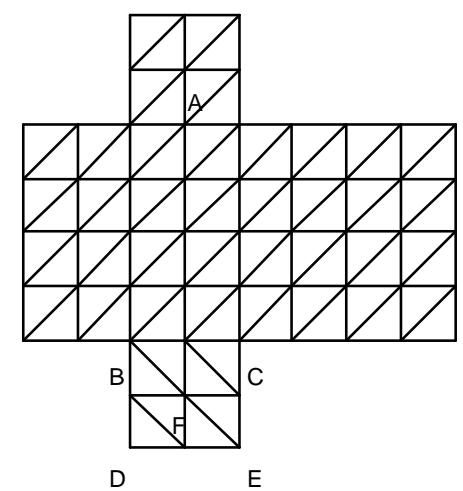

(b)

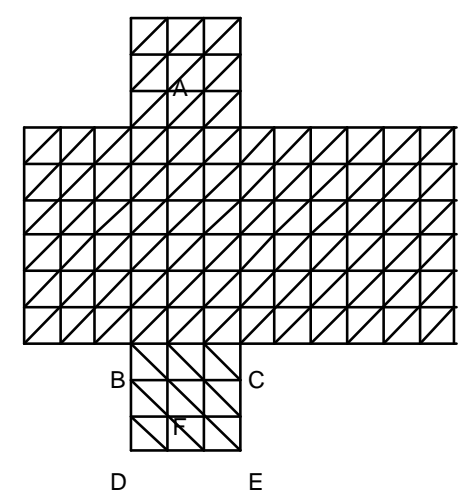

(c)

Figura 7.2 - Discretizações do exemplo 1 
A seguir é apresentado o gráfico 7.1, o qual mostra a convergência do elemento constante para o deslocamento axial do ponto A e o gráfico 7.2 o qual mostra a estabilidade da solução, através do MEC clássico, fazendo-se variar a posição do ponto fonte para as três discretizações apresentadas e comparando-se os resultados com os valores analíticos obtidos em Timoshenko \& Goodier (1970). Dessa forma é possível dizer que os valores encontrados como resposta do problema são estáveis apenas para 240 GL ou mais. Quando as soluções semelhantes ao gráfico 7.2 não apresentarem variação significativa quando se varia a posição do ponto fonte (colocado para fora) entre 0,01L e 0,2L, e o seu valor convergir para a solução analítica, pode-se dizer que se obteve um nível satisfatório de discretização. Sendo assim apresenta-se na tabela 7.1 os resultados de deslocamentos axiais no eixo central do sólido e seus respectivos erros para a discretização com 80 elementos, utilizando-se um fator de 0,1 para a técnica do ponto fora.

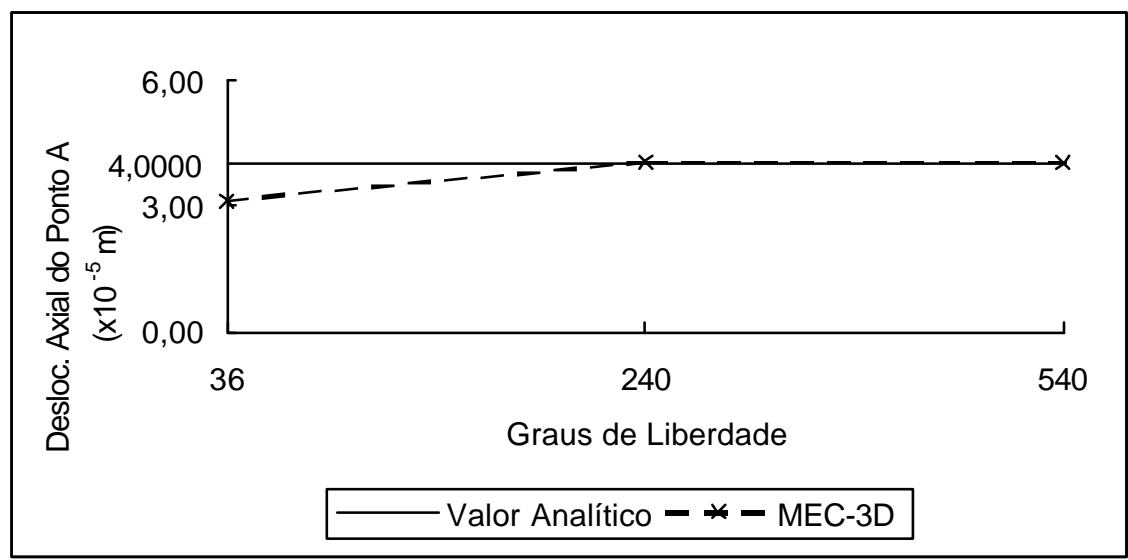

Gráfico 7.1 - Convergência do elemento constante - exemplo 1

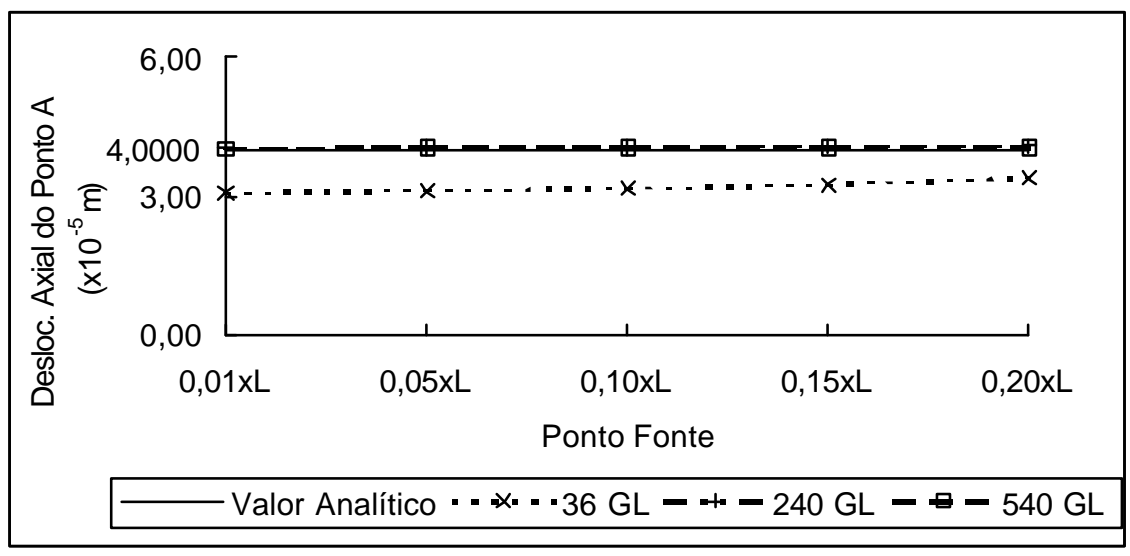

Gráfico 7.2 - Estabilidade da solução para o elemento constante - exemplo 1 
Tabela 7.1 - Deslocamentos axiais com elemento constante (80 elementos - $240 \mathrm{GL}$ )

\begin{tabular}{cccccccc}
\hline COORD. DESLOC. ANALÍTICO & \multicolumn{7}{c}{ FORMULAÇÃO } \\
X3 $(\mathrm{m})$ & PONTO A $\left(\times 10^{-5} \mathrm{~m}\right)$ & ISA & ERRO (\%) & ISSA & ERRO (\%) & ISQG & ERRO (\%) \\
\hline & & & & & & & \\
1,00 & 1,0000 & 0,9992 & $-0,08$ & 1,0007 & 0,07 & 1,0021 & 0,21 \\
2,00 & 2,0000 & 1,9943 & $-0,29$ & 1,9975 & $-0,12$ & 2,0013 & 0,07 \\
3,00 & 3,0000 & 2,9882 & $-0,39$ & 2,9915 & $-0,28$ & 3,0031 & 0,10 \\
4,00 & 4,0000 & 4,0153 & 0,38 & 4,0184 & 0,46 & 4,0431 & 1,08 \\
\hline
\end{tabular}

ISA - INTEGRAL SINGULAR CALCULADA ANALITICAMENTE ISSA - INTEGRAL SINGULAR CALC. SEMI-ANALITICAMENTE

ISQG - INTEGRAL QUASE SINGULAR CALC. NUM. COM QUADRATURA DE GAUSS E SUBDIVISÃO PROGRESSIVA

Apresenta-se na tabela 7.2 as reações dos apoios da base do sólido.

Tabela 7.2 - Reações de apoios com elemento constante (80 elementos - $240 \mathrm{GL}$ )

\begin{tabular}{cccccccc}
\hline ELEMENTO & $\begin{array}{c}\text { REAÇÃO ANALÍTICA } \\
\text { EM X } \text { X }_{3}(\mathrm{~Pa})\end{array}$ & ISA & ERRO (\%) & ISSA & ERRO (\%) & ISQG & ERRO (\%) \\
\hline & 1,0000 & 1,0527 & 5,27 & 1,0537 & 5,37 & 1,0581 & 5,81 \\
1 & 1,0000 & 0,9671 & $-3,29$ & 0,9681 & $-3,19$ & 0,9758 & $-2,42$ \\
2 & 1,0000 & 0,9339 & $-6,61$ & 0,9350 & $-6,50$ & 0,8883 & $-11,17$ \\
3 & 1,0000 & 1,0432 & 4,32 & 1,0442 & 4,42 & 1,0788 & 7,88 \\
4 & & & & & & & \\
\hline
\end{tabular}

ISA - INTEGRAL SINGULAR CALCULADA ANALITICAMENTE ISSA - INTEGRAL SINGULAR CALC. SEMI-ANALITICAMENTE

ISQG - INTEGRAL QUASE SINGULAR CALC. NUM. COM QUADRATURA DE GAUSS E SUBDIVISÃO PROGRESSIVA

A seguir são feitas as mesmas considerações para o elemento linear, primeiro apresenta-se o gráfico 7.3 sobre o qual se faz variar o ponto fonte para verificar a estabilidade da solução e a seguir a tabela 7.3 que mostra os valores de deslocamentos e a tabela 7.4 que apresenta as reações nos apoios da base. $O$ fator utilizado para a técnica do ponto fora é igual a 0,1 . 


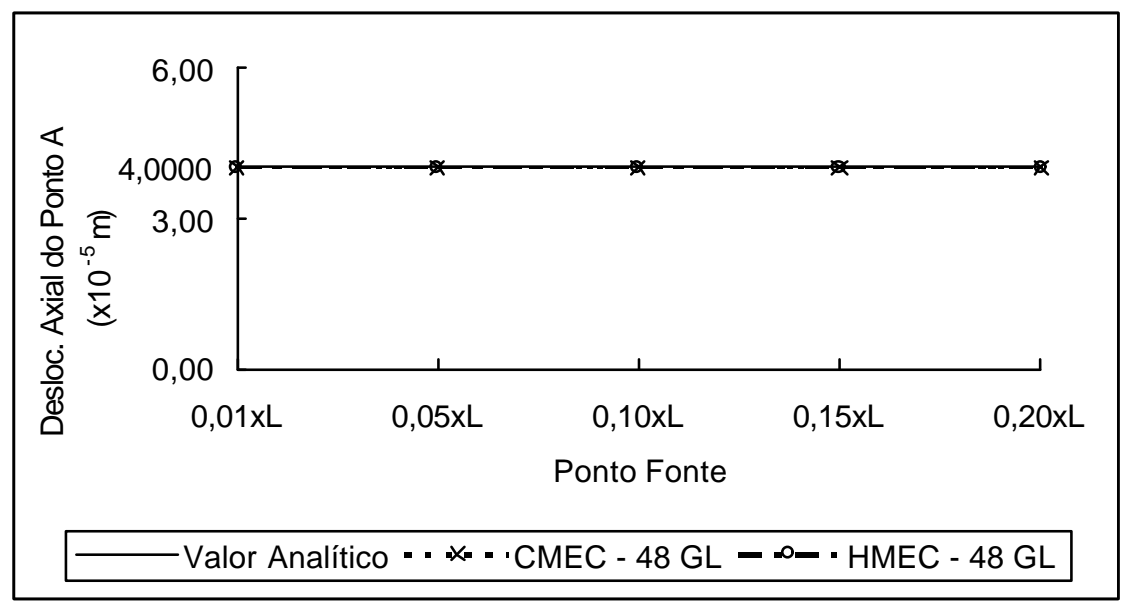

Gráfico 7.3 - Estabilidade da solução para o elemento linear - exemplo 1

Tabela 7.3 - Deslocamentos axiais com elemento linear (12 elementos - $48 \mathrm{GL}$ )

\begin{tabular}{cccccccc}
\hline COORD. & DESLOC. ANALÍTICO & \multicolumn{5}{c}{ FORMULAÇÃO } \\
X3 $(\mathrm{m})$ & PONTO A $\left(\times 10^{-5} \mathrm{~m}\right)$ & ISSA & ERRO $(\%)$ & ISQG & ERRO $\%)$ & HMEC & ERRO (\%) \\
\hline & & & & & & & \\
1,00 & 1,0000 & 1,0000 & 0,00 & 1,0000 & 0,00 & 1,0000 & 0,00 \\
2,00 & 2,0000 & 2,0000 & 0,00 & 2,0000 & 0,00 & 1,9999 & 0,00 \\
3,00 & 3,0000 & 3,0000 & 0,00 & 3,0000 & 0,00 & 2,9999 & 0,00 \\
4,00 & 4,0000 & 4,0000 & 0,00 & 4,0000 & 0,00 & 4,0000 & 0,00 \\
\hline
\end{tabular}

ISSA - INTEGRAL SINGULAR CALC. SEMI-ANALITICAMENTE

ISQG - INTEGRAL QUASE SINGULAR CALC. NUM. COM QUADRATURA DE GAUSS E SUBDIVISÃO PROGRESSIVA HMEC - MEC HÍBRIDO

Tabela 7.4 - Reações de apoios com elemento linear (12 elementos - $48 \mathrm{GL}$ )

\begin{tabular}{cccccccc}
\hline PONTO & $\begin{array}{c}\text { REAÇÃO ANALITICA } \\
\text { EM X } \text { X }_{3}(\mathrm{~Pa})\end{array}$ & ISSA & ERRO (\%) & ISQG & ERRO (\%) & HMEC & ERRO (\%) \\
\hline & & & & & & & \\
B & 1,0000 & 1,0000 & 0,00 & 1,0000 & 0,00 & 1,0000 & 0,00 \\
C & 1,0000 & 1,0000 & 0,00 & 1,0001 & 0,01 & 1,0002 & 0,02 \\
D & 1,0000 & 1,0000 & 0,00 & 1,0000 & 0,00 & 1,0000 & 0,00 \\
E & 1,0000 & 1,0000 & 0,00 & 1,0000 & 0,00 & 1,0000 & 0,00 \\
\hline
\end{tabular}

Deve-se comentar que o fato da aproximação empregada para deslocamento coincidir com o comportamento da resposta analítica conduz àprecisão encontrada para todos os tipos de pontos de colocação, posição e solução fundamental utilizada. Também justifica o uso de apenas 12 elementos. Os erros apresentados nas tabelas 7.3 e 7.4 são numéricos e não de aproximação. 
Para o elemento quadrático é realizada a mesma análise anterior, apresentando no gráfico 7.4 a estabilidade da solução com a variação do ponto fonte, na tabela 7.5 são apresentados os resultados de deslocamentos e na tabela 7.6 as reações de apoio da base. Utiliza-se um fator de 0,1 para a técnica do ponto fora.

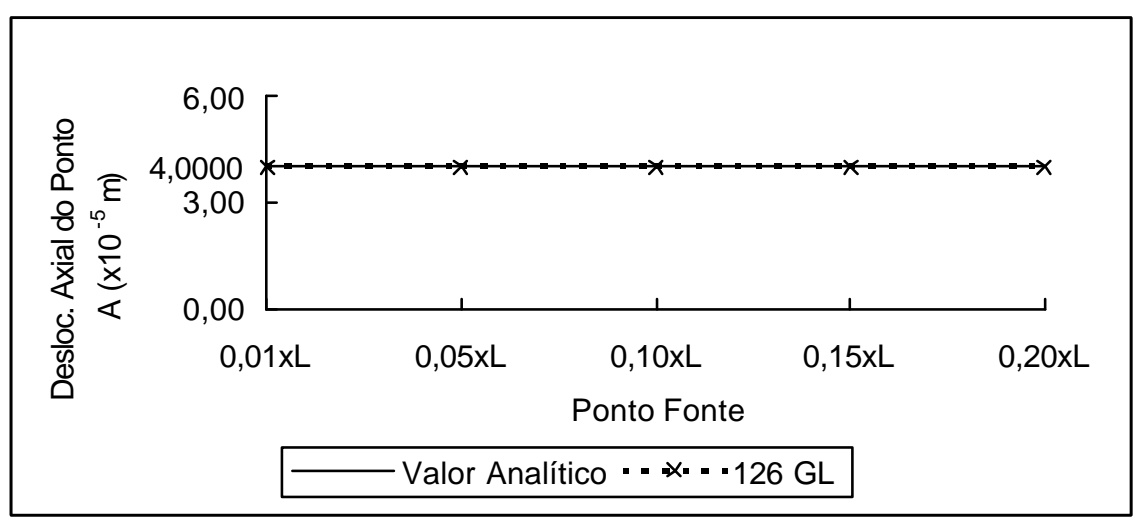

Gráfico 7.4 - Estabilidade da solução para o elemento quadrático - exemplo 1

Tabela 7.5 - Deslocamentos axiais com elemento quadrático (12 elementos - $126 \mathrm{GL}$ )

\begin{tabular}{cccccc}
\hline COORD. & DESLOC. ANALÍTICO & \multicolumn{4}{c}{ FORMULAÇÃO } \\
$\mathrm{X}_{3}(\mathrm{~m})$ & PONTO A $\left(\times 10^{-5} \mathrm{~m}\right)$ & ISSA & ERRO $(\%)$ & ISQG & ERRO $(\%)$ \\
\hline & & & & & \\
1,00 & 1,0000 & 1,0000 & 0,00 & 1,0001 & 0,01 \\
2,00 & 2,0000 & 2,0000 & 0,00 & 2,0001 & 0,01 \\
3,00 & 3,0000 & 3,0000 & 0,00 & 3,0000 & 0,00 \\
4,00 & 4,0000 & 4,0000 & 0,00 & 3,9999 & 0,00 \\
\hline
\end{tabular}

ISSA - INTEGRAL SINGULAR CALC. SEMI-ANALITICAMENTE

ISQG - INTEGRAL QUASE SINGULAR CALC. NUM. COM QUAD. DE GAUSS E SUBDIVISÃO PROGRESSIVA

Tabela 7.6 - Reações de apoios com elemento quadrático (12 elementos - 126 GL)

\begin{tabular}{cccccc}
\hline PONTO & $\begin{array}{c}\text { REAÇÃO ANALITICA } \\
\text { EM } X_{3}(\mathrm{~Pa})\end{array}$ & ISSA & ERRO (\%) & ISQG & ERRO (\%) \\
\hline & & & & & \\
B & 1,0000 & 1,0000 & 0,00 & 0,9979 & $-0,21$ \\
C & 1,0000 & 1,0000 & 0,00 & 0,9999 & $-0,01$ \\
D & 1,0000 & 1,0000 & 0,00 & 0,9994 & $-0,06$ \\
E & 1,0000 & 1,0000 & 0,00 & 0,9998 & $-0,02$ \\
F & 1,0000 & 1,0000 & 0,00 & 1,0001 & 0,01 \\
\hline
\end{tabular}

Como pode ser visto nas tabelas 7.3 e 7.5, os elementos linear e quadrático, respectivamente, apresentam resultados de deslocamentos idênticos aos analíticos, 
o que já era esperado, pois o deslocamento axial varia linearmente com a altura; o pequeno erro apresentado é devido ao cálculo numérico. Para o elemento constante, a tabela 7.1 mostra que o erro é ligeiramente maior, pois cada trecho da altura é representado por um valor constante, além de que para se obter 0 deslocamento do ponto $A$ é necessário fazer uma média dos elementos centrais, diferente dos elementos linear e quadrático, os quais pode-se facilmente discretizar o corpo de tal forma que um nó esteja geometricamente no local do ponto A. Para se obter um resultado ainda melhor seria necessário discretizar ainda mais 0 contorno do sólido, o que não é preciso, pois a solução já atingiu um nível satisfatório de estabilidade e precisão.

As soluções para as reações de apoio para os elementos linear e quadrático também foram idênticas ̀̀ respostas analíticas, conforme pode ser visto nas tabelas 7.4 e 7.6, respectivamente. Para o elemento constante foi constatado um erro um pouco maior, mostrando que a discretização poderia ser um pouco mais densa para que esse erro diminuísse.

Apresenta-se a seguir uma tabela com o número de condicionamento da matriz do sistema linear principal (matriz $\mathrm{A}$ com as colunas já trocadas).

O número de condicionamento diz o quanto a matriz está inconsistente, ou seja, mostra o grau de singularidade existente na matriz, quanto maior o número de condicionamento mais inconsistente é o sistema linear.

Tabela 7.7 - Número de condicionamento - exemplo 1 - elemento constante

\begin{tabular}{lr}
\hline FORMULAÇÃO & $\begin{array}{r}\text { NÚMERO DE CONDICIONAMENTO } \\
\text { 80 ELEMENTOS - } 240 \mathrm{GL}\end{array}$ \\
\hline & \\
ISA & 228.443 \\
ISSA & 228.363 \\
ISQG & 276.253 \\
\hline
\end{tabular}

Para o elemento constante, tabela 7.7 , é visível que o número de condicionamento da técnica que utiliza a quadratura de Gauss para calcular a integral quase singular (ISQG) é um pouco maior, gerando um sistema menos consistente que os outros dois. 
Tabela 7.8 - Número de condicionamento - exemplo 1 - elemento linear

\begin{tabular}{cr}
\hline FORMULAÇÃO & \multicolumn{2}{c}{ NÚMERO DE CONDICIONAMENTO } \\
& 12 ELEMENTOS - 48 GL \\
\hline ISSA & 693.140 \\
ISQG & 1.543 .136 \\
HMEC & 1.628 .071 \\
\hline
\end{tabular}

De acordo com a tabela 7.8, o sistema linear fica mais consistente com a formulação ISSA, o que não significa que os resultados sejam melhores, pois são muito próximos. Quanto às formulações, o HMEC mantém o mesmo nível de consistência da matriz.

Tabela 7.9 - Número de condicionamento - exemplo 1 - elemento quadrático

\begin{tabular}{|c|c|}
\hline FORMULAÇÃO & $\begin{array}{c}\text { NÚMERO DE } \\
\text { CONDICIONAMENTO } \\
12 \text { ELEMENTOS - } 126 \mathrm{GL} \\
\end{array}$ \\
\hline ISSA & 34.795 .529 \\
\hline ISQG & 5.735 .974 .247 \\
\hline
\end{tabular}

$\mathrm{Na}$ tentativa de melhorar o condicionamento do sistema linear testou-se uma nova formulação, cujo desenvolvimento está no anexo C. Trata-se de aplicar as forças pontualmente e não superficialmente como no MEC clássico, assim o que se obtém são reações concentradas, esta formulação apresenta aproximação linear para deslocamentos. Verificou-se a convergência desta formulação para 0 deslocamento axial no ponto $A$, como apresentado no gráfico 7.5 e tabela 7.10. $\mathrm{Na}$ tabela 7.11 são mostrados os números de condicionamento para cada discretização. Utiliza-se a técnica do ponto fora com fator 0,1 . Procurou-se manter a discretização nas faces e aumentar gradativamente no topo e na base do sólido, conforme figura 7.3.

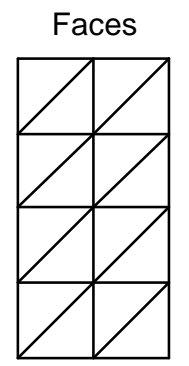

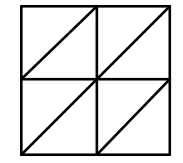

$2 \times 2$

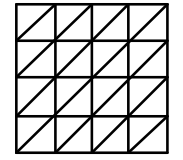

$4 \times 4$
Topo e Base

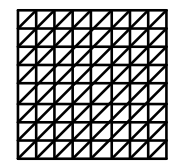

$8 \times 8$

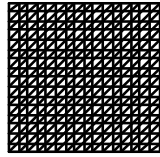

$16 \times 16$

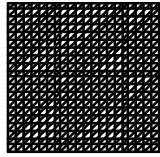

$20 \times 20$

Figura 7.3 - Discretizações da formulação de forças concentradas 


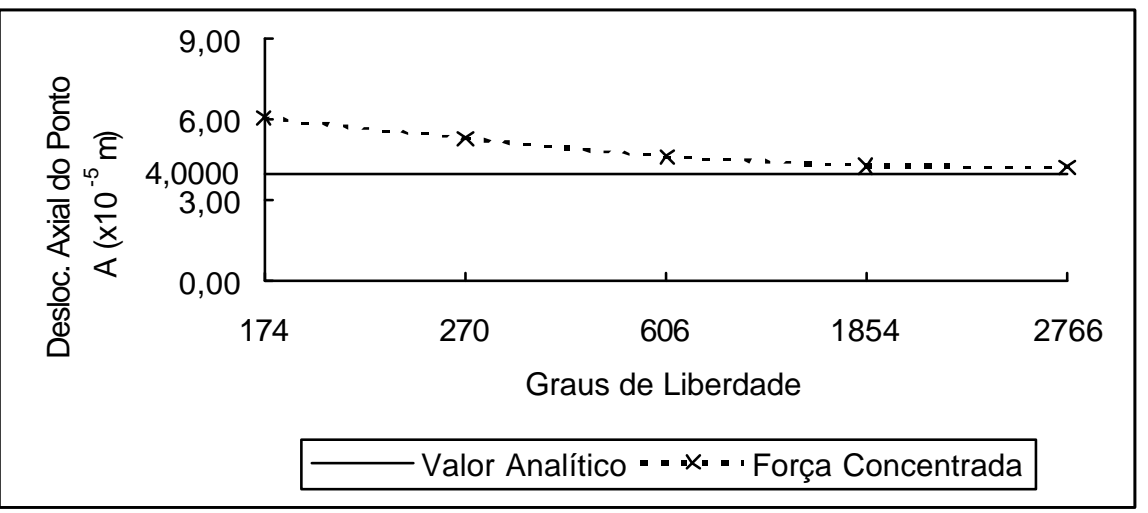

Gráfico 7.5 - Convergência da formulação de forças concentradas

Tabela 7.10 - Deslocamentos obtidos com forças concentradas

\begin{tabular}{cccr}
\hline GRAUS DE & DESLOC. ANALÍTICO & \multicolumn{2}{c}{ FORMULAÇÃO } \\
LIBERDADE & PONTO A $\left(\times 10^{-5} \mathrm{~m}\right)$ & FC & \multicolumn{1}{c}{ ERRO $(\%)$} \\
\hline & & & \\
174 & 4,0000 & 6,0593 & 51,48 \\
270 & 4,0000 & 5,3102 & 32,76 \\
606 & 4,0000 & 4,6212 & 15,53 \\
1854 & 4,0000 & 4,2978 & 7,44 \\
2766 & 4,0000 & 4,2634 & 6,59 \\
& & & \\
\hline
\end{tabular}

FC - FORMULAÇÃO COM FORÇAS CONCENTRADAS

Tabela 7.11 - Número de condicionamento para a formulação de forças concentradas

\begin{tabular}{cr}
\hline GRAUS DE & \multicolumn{2}{c}{ NÚMERO DE } \\
LIBERDADE & CONDICIONAMENTO \\
\hline & \\
174 & 111.414 \\
270 & 315.889 \\
606 & 525.672 \\
1854 & 4.445 .563 \\
2766 & 6.527 .251 \\
& \\
\hline
\end{tabular}

Esta formulação apresentou convergência, como já era esperado, e o número de condicionamento é bem menor, considerando o número de graus de liberdade, se comparado com o elemento linear, mas para obter resultados satisfatórios é preciso aumentar a discretização, o que aumenta o número de graus de liberdade, aumentando assim o tempo de processamento. Desta maneira optou-se por abandonar esta formulação, por ser pouco eficiente para as análises propostas nesta pesquisa. 
Além disso, conclui-se que a consistência do sistema linear não tem influência significativa nos resultados.

\subsection{Exemplo 2 - Sólido tridimensional sob força transversal}

Neste exemplo considera-se de um sólido tridimensional com base quadrangular vinculada nas três direções em cada ponto, submetido a uma força transversal na face superior. As propriedades geométricas e físicas do sólido podem ser vistas na figura 7.4, assim como a configuração adotada para a resolução do problema pelo MEC, que consiste em substituir a força concentrada por uma força distribuída sobre a área do topo do sólido.

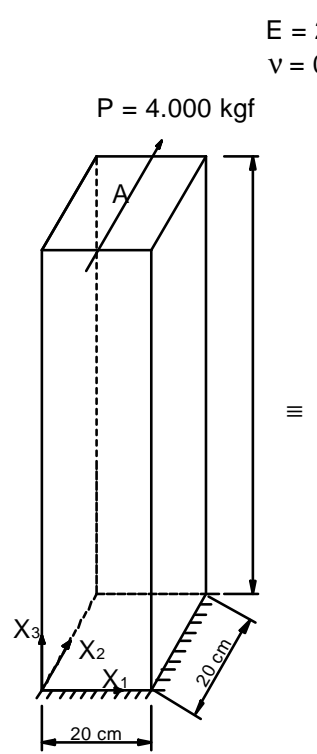

(a)

$$
\begin{aligned}
& E=210.000 \mathrm{kgf} / \mathrm{cm}^{2} \\
& V=0,30
\end{aligned}
$$

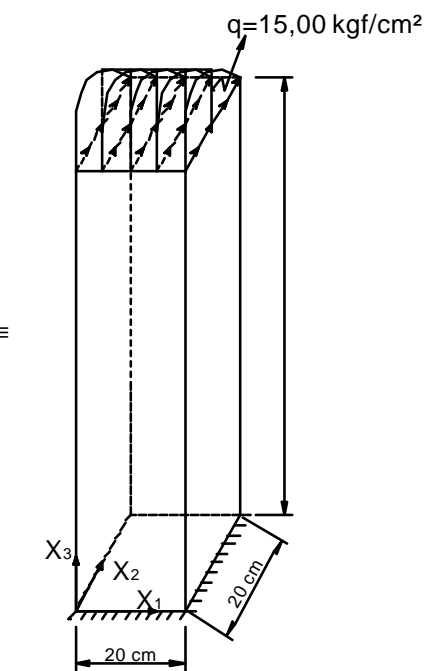

(b)

Figura 7.4 - Sólido tridimensional submetido a uma força transversal - (a) problema clássico e (b) problema equivalente solucionado pelo MEC

Utilizam-se as discretizações mostradas na figura 7.5 para o elemento constante, como para este elemento não é possível aplicar a força como na figura 7.4 (b), procedeu-se à aplicação de forças distribuídas de mesmo valor nos elementos da extremidade. Apresenta-se o gráfico 7.6 de convergência, o gráfico 7.7 de estabilidade e a tabela 7.12 com os resultados de deslocamentos transversais $\left(e m \mathrm{X}_{2}\right)$ dos pontos localizados no eixo central para o elemento constante, comparando-se com valores clássicos obtidos em Schiel (1984), considerando-se a deformação devido ao cisalhamento (demonstrado no apêndice 
C). O valor de deslocamento do ponto A é obtido através da média dos deslocamentos dos elementos mais próximos a este ponto. Utilizou-se o fator para a técnica do ponto fora igual a 0,2 , por este ser um valor que apresenta resultados aceitáveis para o elemento constante.
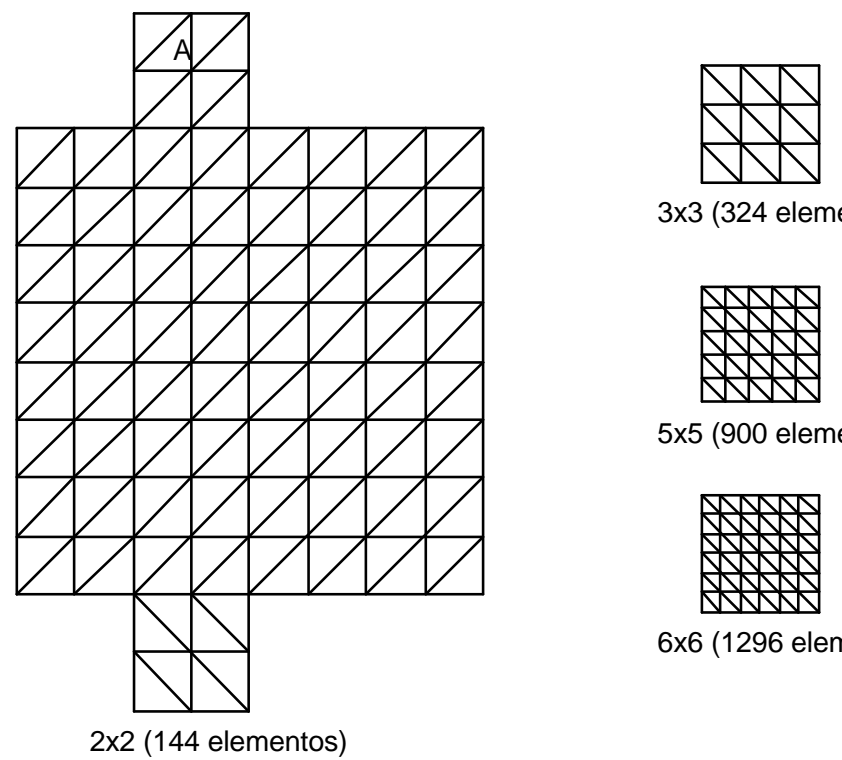

$3 \times 3$ (324 elementos)

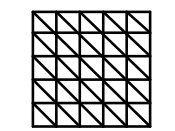

$5 \times 5$ (900 elementos)

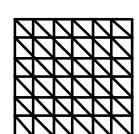

$6 \times 6$ (1296 elementos)

$2 \times 2$ (144 elementos)

Figura 7.5 - Discretizações do sólido do exemplo 2 - elemento constante

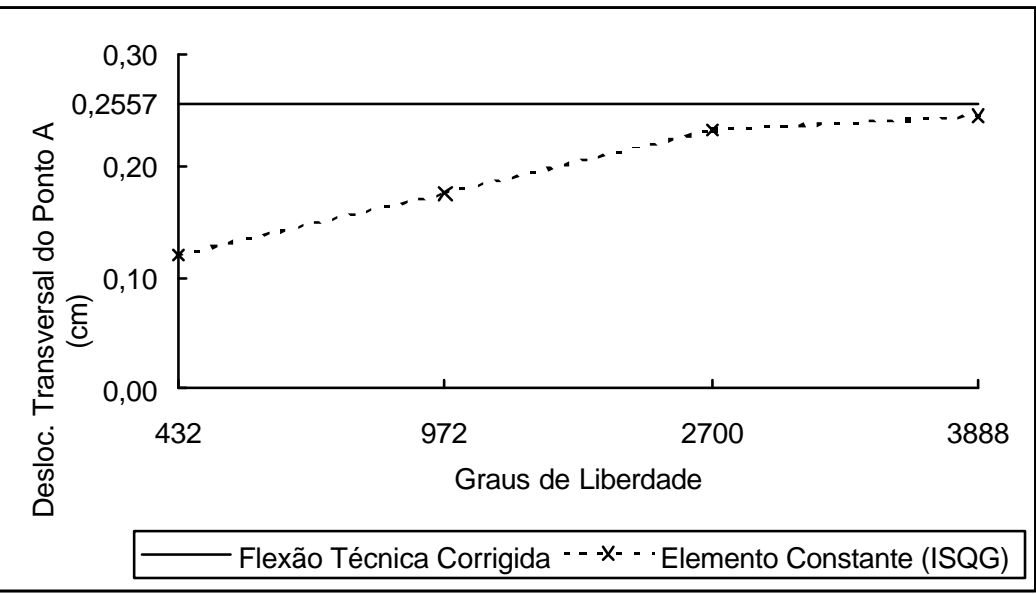

Gráfico 7.6 - Convergência do elemento constante - exemplo 2 


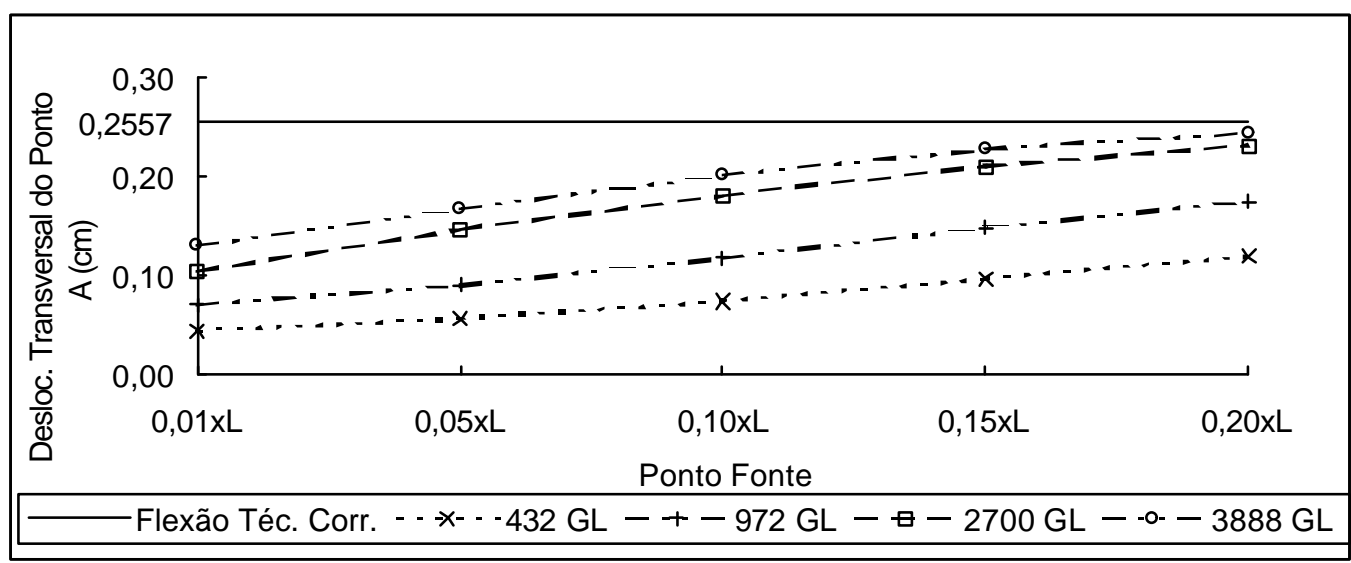

Gráfico 7.7 - Estabilidade da solução para o elemento constante - exemplo 2

Este gráfico mostra que, apesar de convergir para a resposta correta o nível de estabilidade da solução via elemento constante está muito aquém do desejado.

Tabela 7.12 - Deslocamentos transversais $\left(X_{2}\right)$ do exemplo 2 com elemento constante 1296 elementos

\begin{tabular}{cccccccc}
\hline COORD. & FLEXÃO TÉC. CORR. & \multicolumn{5}{c}{ FORMULAÇÃO } \\
$\mathrm{X}_{3}(\mathrm{~cm})$ & DESLOC. EM $X_{2}(\mathrm{~cm})$ & ISA & ERRO $(\%)$ & ISSA & ERRO $(\%)$ & ISQG & ERRO (\%) \\
\hline & & & & & & & \\
20,00 & 0,0239 & 0,0120 & $-49,80$ & 0,0127 & $-46,84$ & 0,0232 & $-3,03$ \\
30,00 & 0,0495 & 0,0239 & $-51,67$ & 0,0253 & $-48,81$ & 0,0479 & $-3,16$ \\
40,00 & 0,0821 & 0,0386 & $-53,06$ & 0,0408 & $-50,28$ & 0,0794 & $-3,29$ \\
50,00 & 0,1205 & 0,0552 & $-54,22$ & 0,0584 & $-51,52$ & 0,1164 & $-3,46$ \\
60,00 & 0,1632 & 0,0730 & $-55,25$ & 0,0773 & $-52,62$ & 0,1572 & $-3,68$ \\
70,00 & 0,2087 & 0,0914 & $-56,20$ & 0,0968 & $-53,65$ & 0,2004 & $-3,97$ \\
80,00 & 0,2557 & 0,1097 & $-57,11$ & 0,1160 & $-54,62$ & 0,2448 & $-4,26$ \\
& & & & & & & \\
\hline
\end{tabular}

Ainda sobre o elemento constante, apresenta-se na tabela 7.13 o número de condicionamento para a discretização com 1296 elementos.

Tabela 7.13 - Número de condicionamento - exemplo 2 - elemento constante

\begin{tabular}{cr}
\hline & \multicolumn{1}{c}{ NÚMERO DE } \\
FORMULAÇÃO & CONDICIONAMENTO \\
& 1296 ELEM. - 3888 GL \\
\hline & \\
ISA & 498.085 \\
ISSA & 503.638 \\
ISQG & 3.290 .595 \\
& \\
\hline
\end{tabular}


No intuito de descobrir porque o ponto externo ao contorno conduz a melhores resultados, apesar de apresentar um condicionamento pior do que o ponto no contorno, estudou-se a sub-matriz $(3 \times 3)$ da diagonal principal na matriz $\mathrm{H}$. Os resultados para um elemento da extremidade estão mostrados no gráfico 7.8.
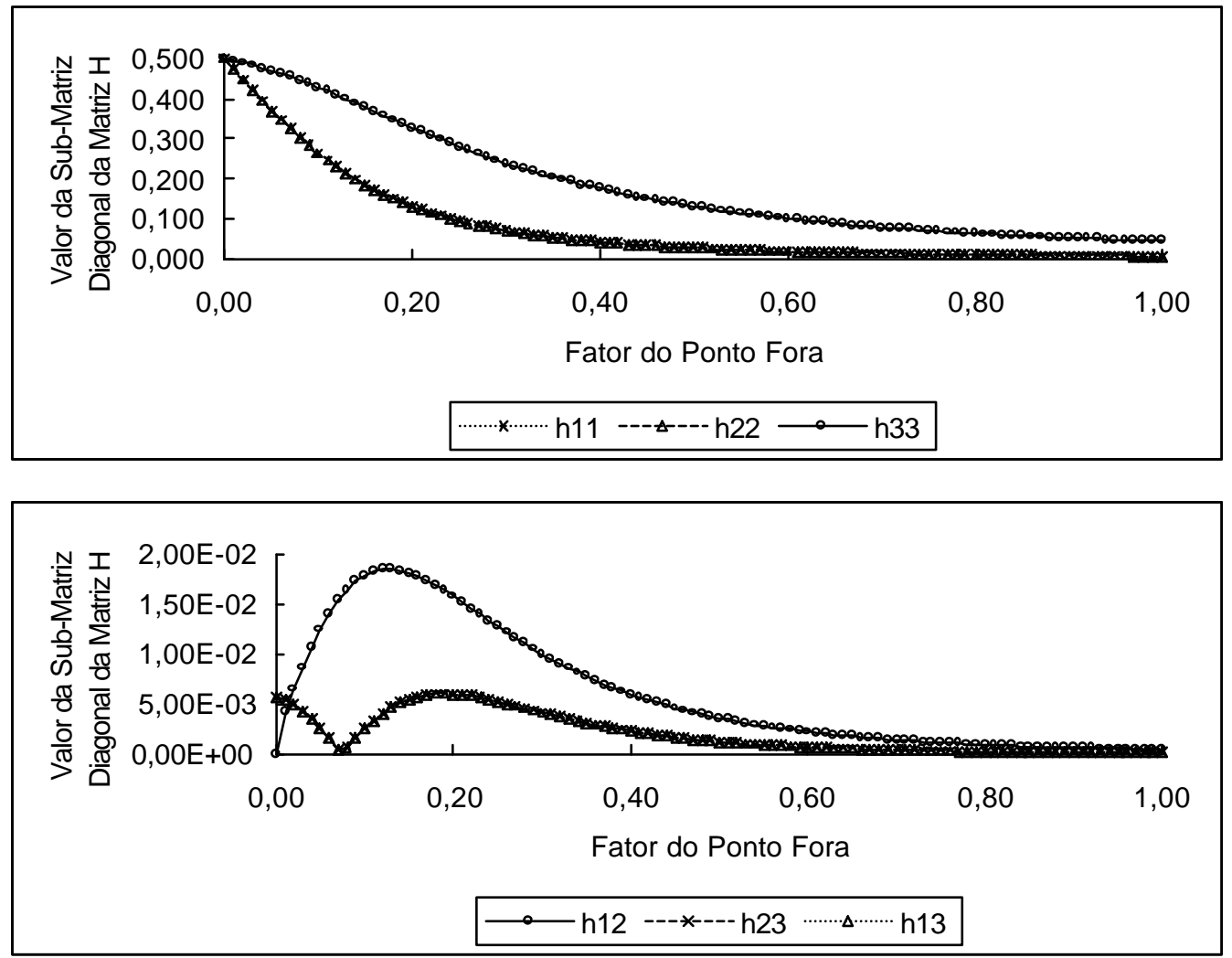

Gráfico 7.8 - Sub-matriz da diagonal principal da matriz H - elemento constante exemplo 2

De acordo com o gráfico 7.8 é possível ver que àmedida que se afasta muito o ponto externo do contorno, o sistema vai ficando mais instável, pois a diagonal principal vai se aproximando de zero. Pode-se ver também que os termos cruzados $\left(h_{12}, h_{13}\right.$ e $\left.h_{23}\right)$ são diferentes de zero quando o ponto é externo ao contorno, principalmente $h_{2}$ que está no plano do elemento, atingindo seu valor máximo quando o ponto fonte está afastado de $0,15 \mathrm{~L}$, por isso a melhor solução ocorrerá ao se escolher uma distância próxima a este valor. Sendo a matriz do sistema linear principal uma matriz diagonal dominante, pois os outros termos possuem valores bem menores que os da diagonal principal, quando tais valores são diferentes de zero conduzem a soluções mais precisas do sistema linear. Plotou-se apenas alguns termos da sub-matriz porque esta é simétrica. 
Para o elemento linear utilizam-se as discretizações mostradas na figura 7.6. Escolheu-se a discretização (a) com 72 elementos (46 nós - $138 \mathrm{GL}$ ), pelo fato desta ter sido utilizada em Barbirato (1999), com isso pode-se comparar os resultados obtidos por este pesquisador e os da presente pesquisa. Para a discretização (a) aplicou-se força distribuída de mesmo valor em todos os nós da extremidade, e nas discretizações (b) e (c) procurou-se aproximar a parábola do carregamento, que é a forma correta de se representar o problema, por trechos lineares.

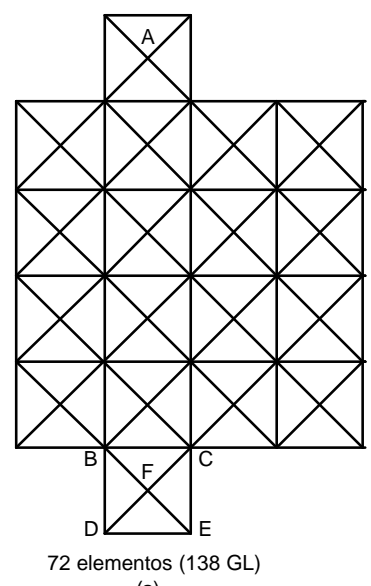

(a)

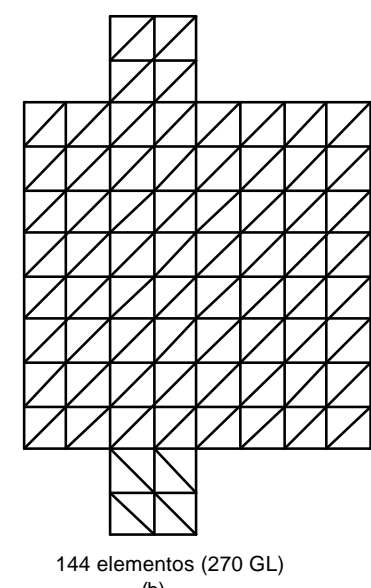

(b)

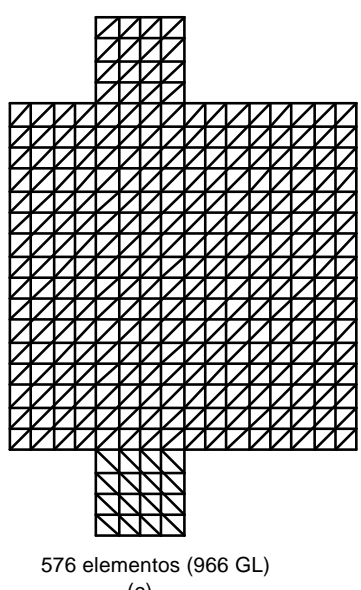

(c)

Figura 7.6 - Discretizações do sólido do exemplo 2 - elemento linear

No gráfico 7.9 mostra-se a estabilidade do MEC clássico para essas discretizações, e no gráfico 7.10 para o HMEC, em seguida apresenta-se a tabela 7.14, a qual contém os valores para deslocamentos transversais (em $X_{2}$ ) para os pontos sobre o eixo central do sólido utilizando-se a discretização (a) com 72 elementos e a tabela 7.15 apresenta os números de condicionamento correspondentes a esta discretização. Utiliza-se $F=0,2$.

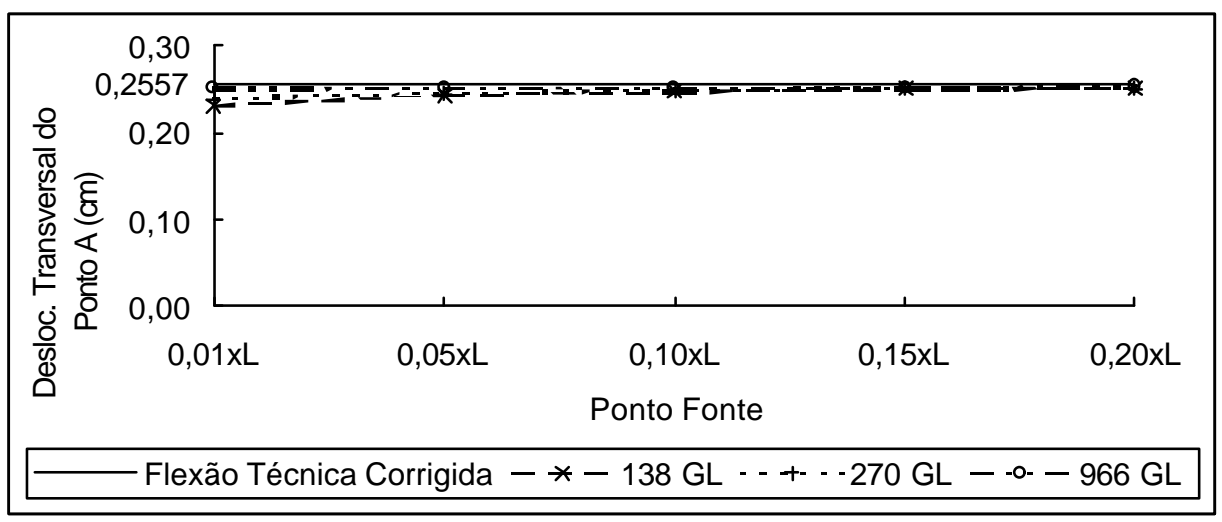

Gráfico 7.9 - Estabilidade da solução para o elemento linear - CMEC - exemplo 2 


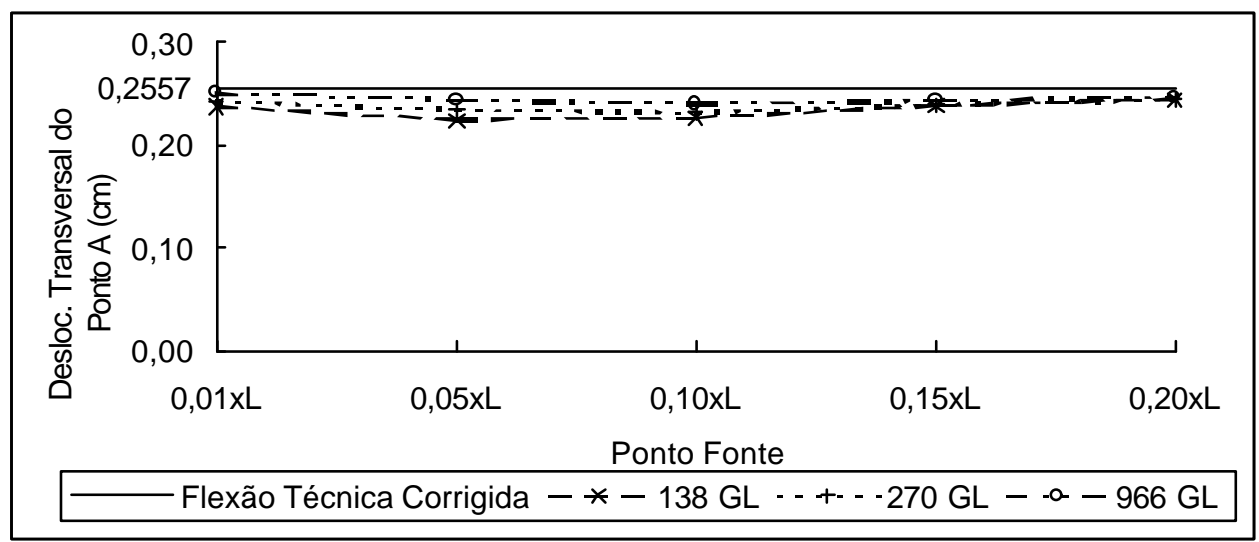

Gráfico 7.10 - Estabilidade da solução para o elemento linear - HMEC - exemplo 2

Tabela 7.14 - Deslocamentos transversais $\left(X_{2}\right)$ do exemplo 2 com elemento linear 72 elementos - $138 \mathrm{GL}$

\begin{tabular}{|c|c|c|c|c|c|c|c|c|c|}
\hline COORD. & FLEXÃO TÉCNICA CORR. & & & & $\overline{\text { FORM }}$ & JLAÇÃO & & & \\
\hline $\mathrm{X}_{3}(\mathrm{~cm})$ & DESLOC. EM X $X_{2}(\mathrm{~cm})$ & ISSA & $\operatorname{ERRO}(\%)$ & ISQG & ERRO $(\%)$ & HMEC & ERRO (\%) & REF. A & $\operatorname{ERRO}(\%)$ \\
\hline 20,0 & 0,0239 & 0,0237 & $-0,81$ & 0,0236 & $-1,50$ & 0,0217 & $-9,31$ & 0,0210 & $-12,22$ \\
\hline 36 & 0 , & 0,0471 & -4 & 0,0490 & $-1,01$ & 0,0463 & $-6,3$ & & \\
\hline 40,00 & 0 & 0,0755 & -8 & 0,0 & $-1,63$ & 0,0773 & $-5,90$ & 0,0740 & $-9,90$ \\
\hline 50,00 & 0 , & 0,1 & -9 , & 0,1 & $-1,59$ & 0,1143 & $-5,19$ & & \\
\hline 60,00 & 0,163 & 0,1444 & $-11,51$ & 0,1602 & $-1,81$ & 0,1551 & $-4,97$ & 0,1490 & $-8,70$ \\
\hline 70,6 & 0,2 & & & & & 0,1989 & -4 & & \\
\hline 80,00 & 0,2557 & 0,2219 & $-13,23$ & 0,2507 & $-1,96$ & 0,2439 & $-4,61$ & 0,2350 & $-8,09$ \\
\hline
\end{tabular}

REF. A - Barbirato (1999)

Tabela 7.15 - Número de condicionamento - exemplo 2 - 138 GL - elemento linear

\begin{tabular}{cr}
\hline \multirow{2}{*}{ FORMULAÇÃO } & \multicolumn{1}{c}{ NÚMERO DE } \\
& CONDICIONAMENTO \\
& 72 ELEMENTOS - 138 GL \\
\hline & \\
ISSA & 448.170 \\
ISQG & 478.690 \\
HMEC & 2.335 .306 \\
\hline
\end{tabular}

Este exemplo serve para demonstrar como a técnica usada neste trabalho, utilizando a quadratura de Gauss com subdivisão progressiva para calcular a integral quase singular, apresenta resultados bem mais próximos dos valores analíticos do que a técnica usual, que é a divisão máxima em 25 subelementos triangulares de mesmo tamanho, utilizando-se a quadratura de Hammer para calcular a integral quase singular (Barbirato 1999). A formulação HMEC apresenta- 
se menos estável, mas ainda assim, conduz a resultados aceitáveis para discretização mais densa.

Na tabela 7.16 estão os deslocamentos para a discretização (b) e na tabela 7.17 os números de condicionamento desta discretização. Na tabela 7.18 apresentam-se os deslocamentos para a discretização (c) e na tabela 7.19 os números de condicionamento correspondentes a esta discretização. Utiliza-se o fator 0,2 para a técnica do ponto fora, pois nesta situação a variabilidade dos resultados é a menor (ver gráfico 7.9).

Tabela 7.16 - Deslocamentos transversais $\left(X_{2}\right)$ do exemplo 2 com elemento linear 144 elementos - $270 \mathrm{GL}$

\begin{tabular}{cccccccc}
\hline \multicolumn{3}{c}{ COORD. FLEXÃO TÉCNICA CORR. } & \multicolumn{7}{c}{ FORMULAÇÃO } \\
$\mathrm{X}_{3}(\mathrm{~cm})$ & DESLOC. EM $\mathrm{X}_{2}(\mathrm{~cm})$ & ISSA & ERRO $(\%)$ & ISQG & ERRO $\%)$ & HMEC & ERRO (\%) \\
\hline & & & & & & & \\
20,00 & 0,0239 & 0,02342 & $-2,12$ & 0,02319 & $-3,08$ & 0,02132 & $-10,90$ \\
30,00 & 0,0495 & 0,04723 & $-4,50$ & 0,04815 & $-2,65$ & 0,04531 & $-8,39$ \\
40,00 & 0,0821 & 0,07717 & $-6,04$ & 0,08017 & $-2,39$ & 0,07640 & $-6,99$ \\
50,00 & 0,1205 & 0,11192 & $-7,14$ & 0,11784 & $-2,23$ & 0,11313 & $-6,13$ \\
60,00 & 0,1632 & 0,15023 & $-7,95$ & 0,15975 & $-2,11$ & 0,15410 & $-5,58$ \\
70,00 & 0,2087 & 0,19092 & $-8,53$ & 0,20450 & $-2,03$ & 0,19791 & $-5,19$ \\
80,00 & 0,2557 & 0,23309 & $-8,84$ & 0,25072 & $-1,95$ & 0,24295 & $-4,98$ \\
\hline
\end{tabular}

Tabela 7.17 - Número de condicionamento - exemplo 2 - 270 GL - elemento linear

\begin{tabular}{|c|c|}
\hline FORMULAÇÃO & $\begin{array}{c}\text { NÚMERO DE } \\
\text { CONDICIONAMENTO } \\
144 \text { ELEMENTOS - } 270 \mathrm{GL}\end{array}$ \\
\hline ISSA & 443.887 \\
\hline ISQG & 3.192 .730 \\
\hline HMEC & 4.272 .518 \\
\hline
\end{tabular}

Tabela 7.18 - Deslocamentos transversais $\left(X_{2}\right)$ do exemplo 2 com elemento linear 576 elementos - $966 \mathrm{GL}$

\begin{tabular}{cccccccc}
\hline $\begin{array}{c}\text { COORD. FLEXÃO TÉCNICA CORR. } \\
\mathrm{X}_{3}(\mathrm{~cm})\end{array}$ & $\begin{array}{c}c \\
\text { DESLOC. EM X }\end{array}(\mathrm{cm})$ & ISSA & ERRO (\%) & ISQG & ERRO $(\%)$ & HMEC & ERRO (\%) \\
\hline \multirow{2}{*}{20,00} & 0,0239 & 0,02340 & $-2,19$ & 0,02323 & $-2,90$ & 0,02161 & $-9,69$ \\
30,00 & 0,0495 & 0,04831 & $-2,32$ & 0,04828 & $-2,39$ & 0,04589 & $-7,22$ \\
40,00 & 0,0821 & 0,08008 & $-2,50$ & 0,08039 & $-2,12$ & 0,07730 & $-5,89$ \\
50,00 & 0,1205 & 0,11729 & $-2,68$ & 0,11815 & $-1,97$ & 0,11441 & $-5,07$ \\
60,00 & 0,1632 & 0,15857 & $-2,84$ & 0,16015 & $-1,87$ & 0,15579 & $-4,54$ \\
70,00 & 0,2087 & 0,20258 & $-2,95$ & 0,20496 & $-1,81$ & 0,20003 & $-4,17$ \\
80,00 & 0,2557 & 0,24806 & $-2,99$ & 0,25130 & $-1,72$ & 0,24580 & $-3,87$ \\
\hline
\end{tabular}


Tabela 7.19 - Número de condicionamento - exemplo 2 - 966 GL - elemento linear

\begin{tabular}{|c|c|}
\hline FORMULAÇÃO & $\begin{array}{c}\text { NÚMERO DE } \\
\text { CONDICIONAMENTO } \\
576 \text { ELEMENTOS - } 966 \text { GL }\end{array}$ \\
\hline ISSA & 703.494 \\
\hline ISQG & 1.810 .593 \\
\hline HMEC & 3.057 .894 \\
\hline
\end{tabular}

Os resultados de deslocamentos com o ponto no contorno mostram que com a discretização (c), além de mais estável, conduz a melhores resultados com o elemento linear, já para o ponto fonte externo ao contorno não houve grande diferença entre as discretizações, assim como para o HMEC.

Verifica-se para o ponto A (extremidade) o comportamento da sub-matriz da diagonal principal da matriz $\mathrm{H}$ para o elemento linear no gráfico 7.11 .
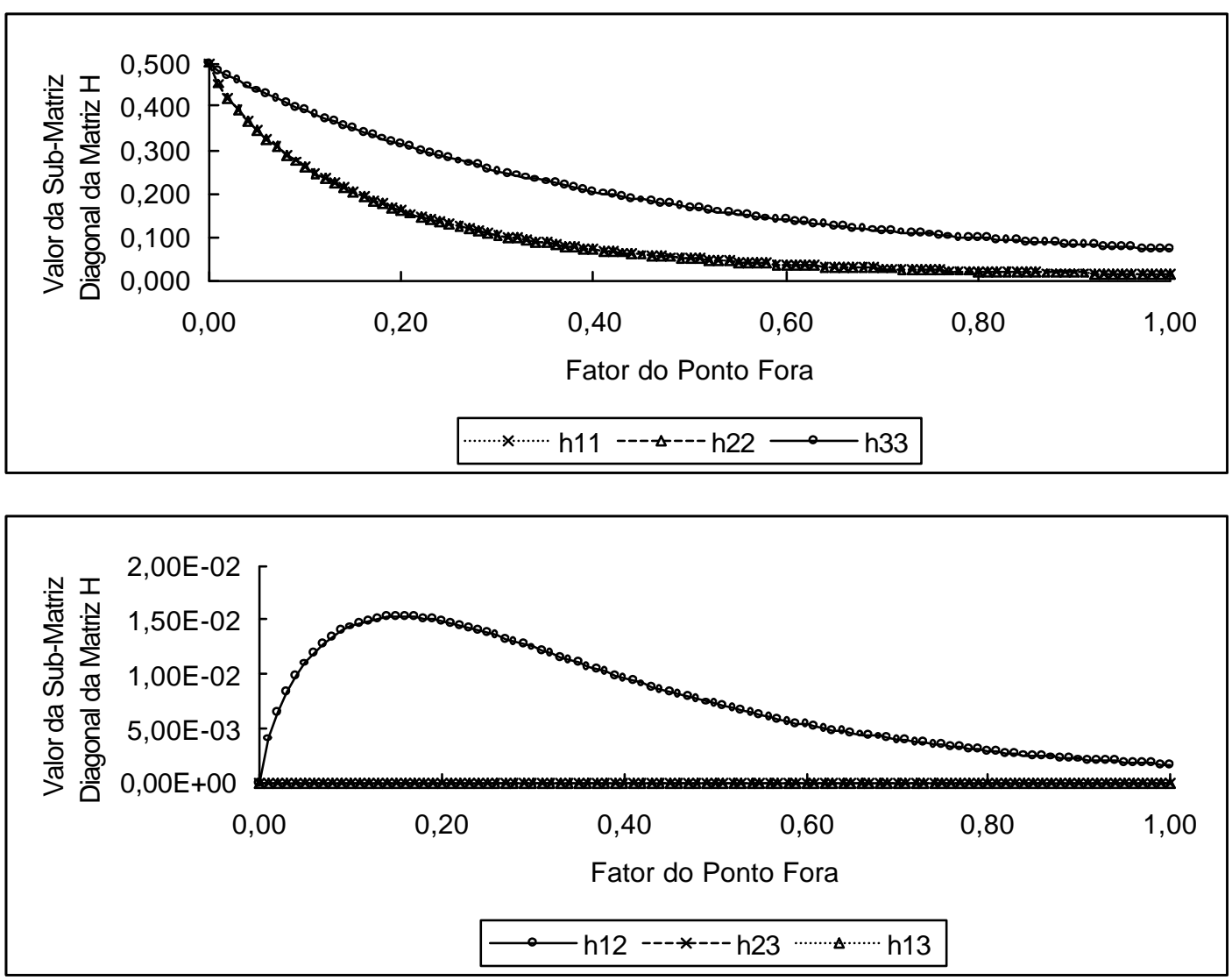

Gráfico 7.11 - Sub-matriz da diagonal principal da matriz H - elemento linear exemplo 2 
Como o elemento constante, o elemento linear também mostra valores diferentes de zero para o termo $h_{2}$, o que leva a melhores resultados em um intervalo próximo ao seu valor de máximo, mas diferentemente do elemento constante os outros termos cruzados são identicamente nulos, como quando o ponto fonte está no contorno, provavelmente seja esta a causa do elemento constante apresentar tamanha diferença entre as formulações, tendo vantagens para a formulação ISQG com ponto fonte externo ao contorno. Resultados de outros pontos sobre o contorno também apresentaram o mesmo comportamento, diferindo apenas no termo que é diferente de zero.

Para o elemento quadrático utiliza-se uma discretização de 36 elementos (90 nós - $270 \mathrm{GL}$ ), conforme mostra a figura 7.7. Em seguida mostra-se o gráfico 7.12 com a estabilidade deste elemento e a tabela 7.20 com os valores de deslocamentos transversais dos pontos situados no eixo central do sólido. A tabela 7.21 mostra a comparação dos números de condicionamento. Utilizou-se um fator de 0,1 para técnica do ponto fora, pois como pode ser visto no gráfico 7.12 , este elemento mostrou-se bastante estável para quaisquer das duas discretizações apresentadas na figura 7.7 .

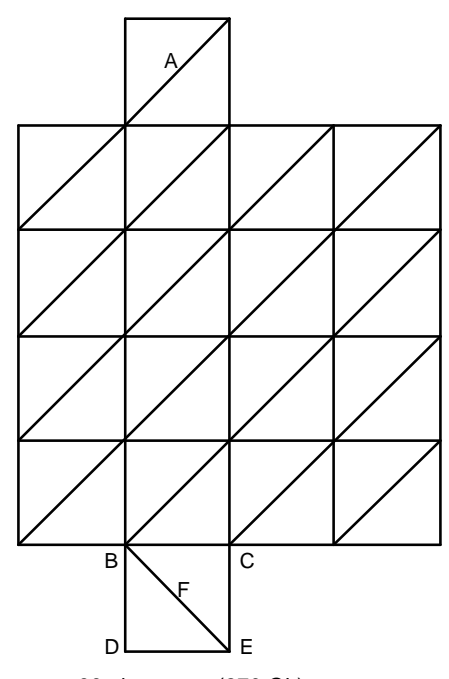

36 elementos ( $270 \mathrm{GL})$

(a)

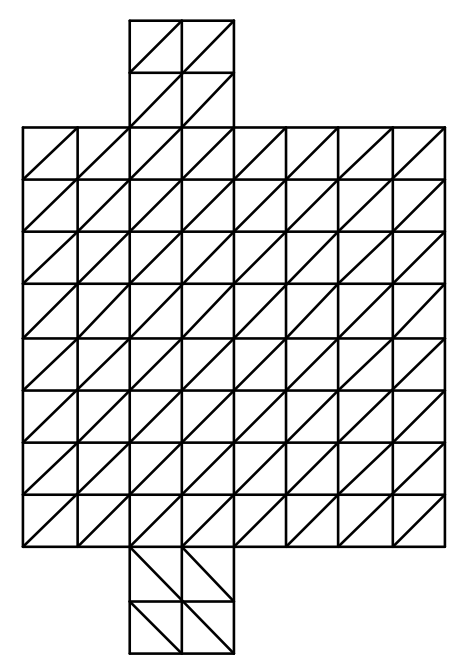

144 elementos (966 GL)

(b)

Figura 7.7 - Discretizações do sólido do exemplo 2 - elemento quadrático 


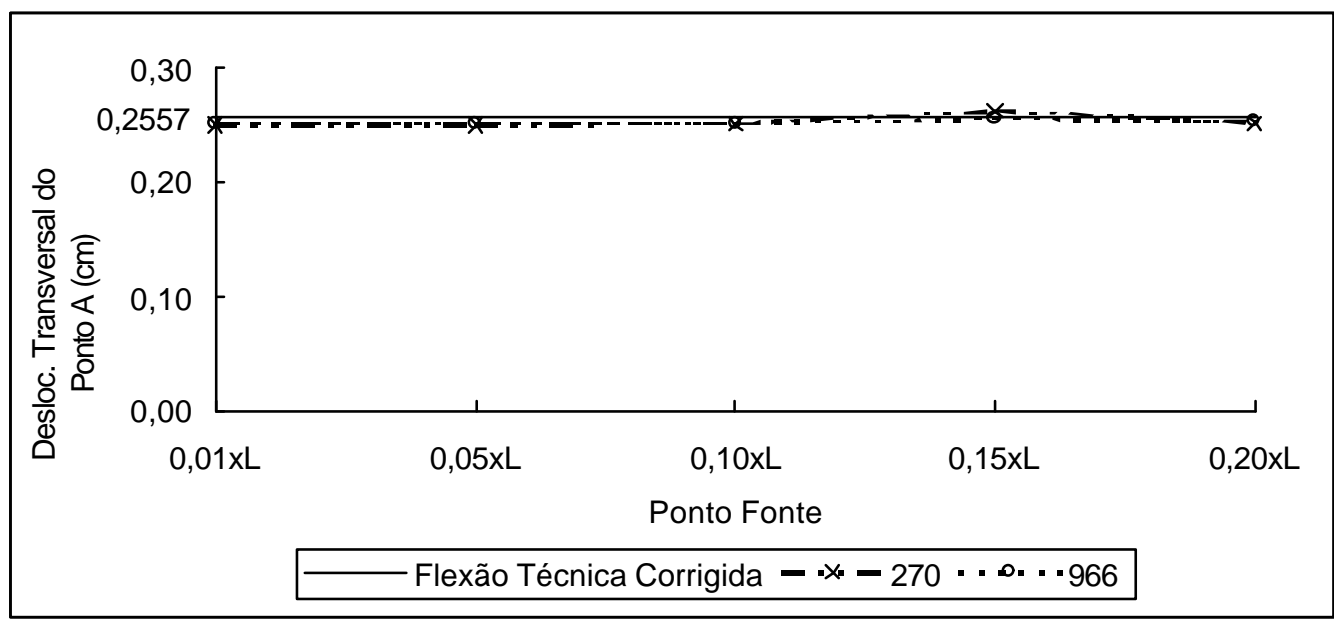

Gráfico 7.12 - Estabilidade da solução para o elemento quadrático - exemplo 2

Tabela 7.20 - Deslocamentos transversais $\left(X_{2}\right)$ do exemplo 2 com elemento quadrático - 36 elementos - $270 \mathrm{GL}$

\begin{tabular}{|c|c|c|c|c|c|}
\hline \multirow{2}{*}{$\begin{array}{l}\text { COORD. } \\
\mathrm{X}_{3}(\mathrm{~cm}) \\
\end{array}$} & \multirow{2}{*}{$\begin{array}{l}\text { FLEXÃO TÉC. CORRIG. } \\
\text { DESLOC. EM X } X_{2}(\mathrm{~cm}) \\
\end{array}$} & \multicolumn{4}{|c|}{ FORMULAÇÃO } \\
\hline & & ISSA & ERRO (\%) & ISQG & ERRO (\%) \\
\hline 20,00 & 0,0239 & 0,02248 & $-6,02$ & 0,02278 & $-4,77$ \\
\hline 30,00 & 0,0495 & 0,04728 & $-4,40$ & 0,04771 & $-3,54$ \\
\hline 40,00 & 0,0821 & 0,07926 & $-3,49$ & 0,07970 & $-2,96$ \\
\hline 50,00 & 0,1205 & 0,11700 & $-2,92$ & 0,11743 & $-2,57$ \\
\hline 60,00 & 0,1632 & 0,15906 & $-2,54$ & 0,15943 & $-2,31$ \\
\hline 70,00 & 0,2087 & 0,20400 & $-2,27$ & 0,20431 & $-2,12$ \\
\hline 80,00 & 0,2557 & 0,25046 & $-2,05$ & 0,25063 & $-1,98$ \\
\hline
\end{tabular}

Tabela 7.21 - Número de condicionamento - exemplo 2 - elemento quadrático

\begin{tabular}{|c|c|}
\hline FORMULAÇÃO & $\begin{array}{c}\text { NÚMERO DE } \\
\text { CONDICIONAMENTO } \\
\text { 36 ELEMENTOS - } 270 \mathrm{GL}\end{array}$ \\
\hline ISSA & 509.556 \\
\hline ISQG & 3.365 .744 \\
\hline
\end{tabular}

O elemento quadrático mostra, como esperado, uma maior estabilidade que os elementos constante e linear. Os valores de deslocamentos para o ponto fonte no contorno são tão bons quanto para o ponto fonte externo ao contorno.

Verifica-se também para o elemento quadrático os termos da sub-matriz da diagonal principal da matriz $\mathrm{H}$, conforme gráfico 7.13 . 

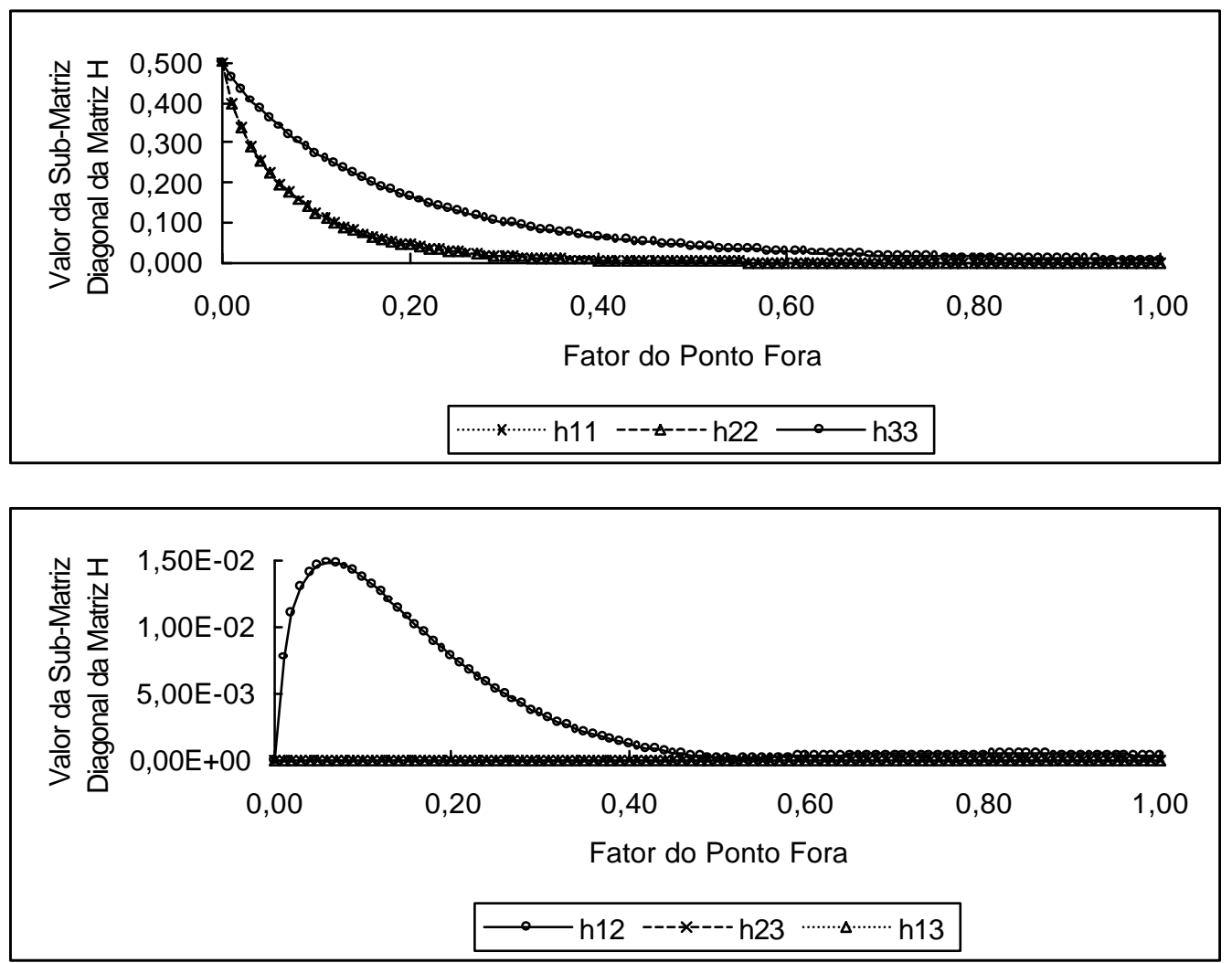

Gráfico 7.13 - Sub-matriz da diagonal principal da matriz H - elemento quadrático exemplo 2

O gráfico 7.13 mostra que o elemento quadrático possui comportamento análogo ao elemento linear, com a diferença que o termo $h_{12}$ possui valor máximo menor do que o seu valor para o elemento linear, e o seu pico é mais concentrado.

Este exemplo mostra a sensibilidade dos elementos de contorno ao tipo de resposta esperada, ou seja, quando se tem resposta linear, o elemento linear já conduz a bons resultados; e quando a resposta é quadrática, o elemento quadrático conduz a melhores resultados. Para que um elemento de grau menor conduza a resultados aceitáveis e estáveis torna-se necessária uma discretização exageradamente grande.

Neste exemplo também é possível verificar para o elemento linear que a formulação clássica (CMEC) leva vantagem sobre a formulação com o uso da função hiper-singular (HMEC). A formulação HMEC apresenta um número de condicionamento bem maior que o do CMEC, tornando o sistema linear mais instável, refletindo ligeiramente nos resultados.

Também se pode notar, através deste exemplo, que o elemento constante apesar de apresentar um condicionamento melhor, conduz a resultados muito 
imprecisos, sendo necessário aumentar exageradamente a discretização para que os resultados apresentem convergência para o ponto fonte colocado $0,2 \mathrm{~L}$ do contorno.

Isto não quer dizer que a resposta seja aceitável, basta ver o gráfico 7.7 que reflete a instabilidade dos resultados. Assim, este elemento deve ser descartado para análises da prática.

\subsection{Exemplo 3 - Sólido sob força transversal com vários comprimentos}

Neste exemplo é considerado o mesmo sólido do exemplo 2, inclusive as propriedades físicas e geométricas, a não ser pela altura, que agora varia de $20 \mathrm{~cm}$ até $200 \mathrm{~cm}$ de $20 \mathrm{em} 20 \mathrm{~cm}$. Para o elemento linear é mantida a mesma densidade de malha da discretização (b) apresentada na figura 7.6, ou seja, o número de elementos varia, assim como o número de nós. São apresentados na tabela 7.22 os resultados de deslocamentos transversais sobre o eixo $X_{\Sigma}$ no ponto $A$ do sólido (extremidade) e na tabela 7.23 os números de condicionamento deste elemento para esta discretização. É mantido o fator de 0,2 para a técnica do ponto fora.

Tabela 7.22 - Deslocamentos transversais $\left(X_{2}\right)$ do exemplo 3 com elemento linear

\begin{tabular}{rcccccccc}
\hline $\begin{array}{c}\text { ALTURA } \\
(\mathrm{cm})\end{array}$ & $\begin{array}{c}\text { RELAÇÃO } \\
\text { H:B }\end{array}$ & $\begin{array}{c}\text { FLEXÃO TÉC. CORR. } \\
\text { DESLOC. EM } X_{2}(\mathrm{~cm})\end{array}$ & ISSA & ERRO (\%) & ISQG & ERRO $(\%)$ & HMEC & ERRO (\%) \\
\hline & & & & & & & & \\
20,00 & $1: 1$ & 0,0068 & 0,0058 & $-14,71$ & 0,0064 & $-5,86$ & 0,00570 & $-15,91$ \\
40,00 & $2: 1$ & 0,0364 & 0,0317 & $-13,04$ & 0,0350 & $-3,83$ & 0,03286 & $-9,78$ \\
60,00 & $3: 1$ & 0,1118 & 0,0996 & $-10,92$ & 0,1088 & $-2,70$ & 0,10429 & $-6,69$ \\
80,00 & $4: 1$ & 0,2557 & 0,2331 & $-8,84$ & 0,2507 & $-1,95$ & 0,24319 & $-4,89$ \\
100,00 & $5: 1$ & 0,4910 & 0,4582 & $-6,69$ & 0,4846 & $-1,32$ & 0,47324 & $-3,63$ \\
120,00 & $6: 1$ & 0,8407 & 0,8031 & $-4,47$ & 0,8347 & $-0,71$ & 0,81887 & $-2,59$ \\
140,00 & $7: 1$ & 1,3275 & 1,2981 & $-2,21$ & 1,3263 & $-0,09$ & 1,30540 & $-1,66$ \\
160,00 & $8: 1$ & 1,9742 & 1,9747 & 0,02 & 1,9857 & 0,58 & 1,95950 & $-0,75$ \\
180,00 & $9: 1$ & 2,8039 & 2,8648 & 2,17 & 2,8408 & 1,32 & 2,80880 & 0,18 \\
200,00 & $10: 1$ & 3,8392 & 4,0000 & 4,19 & 3,9209 & 2,13 & 3,88320 & 1,15 \\
& & & & & & & & \\
\hline
\end{tabular}


Tabela 7.23 - Número de condicionamento - exemplo 3 - elemento linear

\begin{tabular}{ccccc}
\hline $\begin{array}{c}\text { ALTURA } \\
(\mathrm{cm})\end{array}$ & $\begin{array}{c}\text { RELAÇÃO } \\
\text { H:B }\end{array}$ & \multicolumn{3}{c}{ NÚMERO DE CONDICIONAMENTO } \\
\hline & & & & \\
& ISSA & ISQG & HMEC \\
20,00 & $1: 1$ & 467.329 & 3.224 .694 & 3.462 .016 \\
40,00 & $2: 1$ & 411.960 & 3.195 .681 & 4.328 .680 \\
60,00 & $3: 1$ & 408.746 & 3.222 .177 & 4.380 .192 \\
80,00 & $4: 1$ & 443.887 & 3.192 .730 & 4.272 .518 \\
100,00 & $5: 1$ & 516.128 & 3.126 .219 & 4.093 .698 \\
120,00 & $6: 1$ & 642.535 & 3.035 .260 & 3.883 .621 \\
140,00 & $7: 1$ & 795.513 & 2.928 .479 & 3.662 .735 \\
160,00 & $8: 1$ & 982.833 & 2.811 .882 & 3.439 .472 \\
180,00 & $9: 1$ & 1.197 .308 & 2.694 .719 & 3.237 .788 \\
200,00 & $10: 1$ & 1.441 .252 & 2.578 .458 & 3.054 .001 \\
& & & & \\
\hline
\end{tabular}

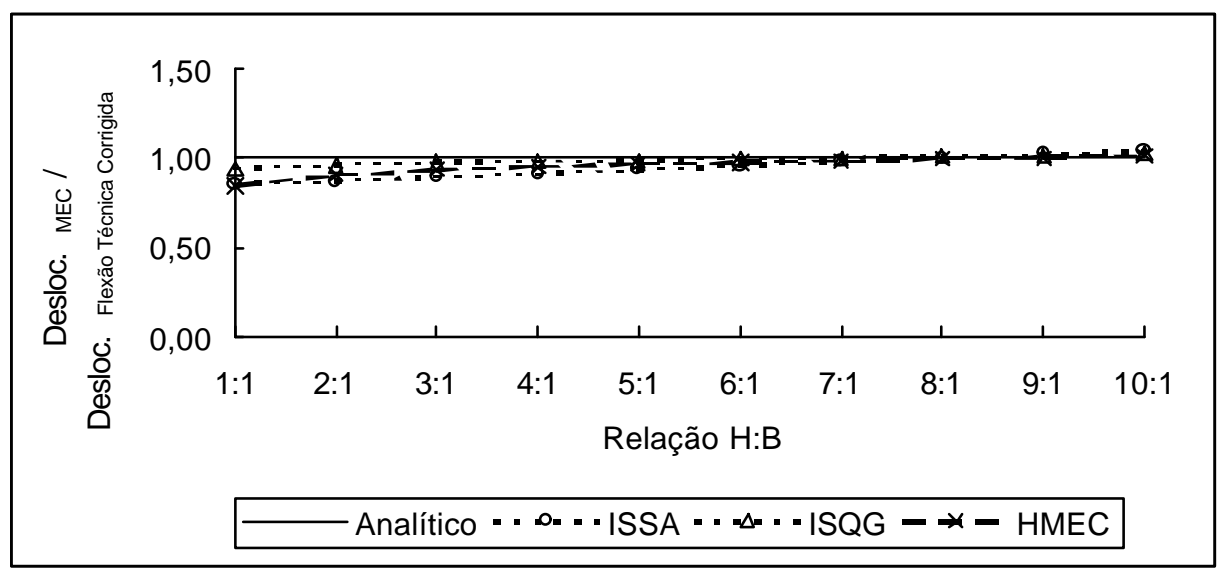

Gráfico 7.14 - Comportamento do deslocamento com a variação do comprimento elemento linear

Os resultados deste exemplo para o elemento linear mostram que a formulação ISQG com o ponto fonte externo ao contorno é melhor para este tipo de problema do que a ISSA com o ponto fonte no contorno e HMEC que também utiliza o ponto fonte externo ao contorno. Mais uma vez o número de condicionamento mostra que o HMEC está produzindo um sistema mais instável.

Para o elemento quadrático, utilizou-se a mesma densidade de malha da discretização apresentada na figura 7.7 (a) para o exemplo anterior. Na tabela 7.24 apresentam-se os deslocamentos transversais do ponto A e na tabela 7.25 , 0 condicionamento deste elemento para cada comprimento e no gráfico 7.15 , o comportamento do deslocamento de acordo com o comprimento. Utiliza-se 0,1 
como fator de ponto fora para este elemento, pois este elemento apresenta-se estável.

Tabela 7.24 - Deslocamentos transversais $\left(X_{2}\right)$ do exemplo 3 com elemento quadrático

\begin{tabular}{rcccccc}
\hline $\begin{array}{c}\text { ALTURA } \\
(\mathrm{cm})\end{array}$ & $\begin{array}{c}\text { RELAÇÃO } \\
\mathrm{H}: \mathrm{B}\end{array}$ & $\begin{array}{c}\text { FLEXÃO TÉC. CORR. } \\
\text { DESLOC. EM X }\end{array}(\mathrm{cm})$ & ISSA & ERRO $(\%)$ & ISQG & ERRO (\%) \\
\hline & & & & & & \\
20,00 & $1: 1$ & 0,0068 & 0,0061 & $-10,33$ & 0,0063 & $-7,12$ \\
40,00 & $2: 1$ & 0,0364 & 0,0345 & $-5,18$ & 0,0350 & $-3,80$ \\
60,00 & $3: 1$ & 0,1118 & 0,1083 & $-3,11$ & 0,1089 & $-2,61$ \\
80,00 & $4: 1$ & 0,2557 & 0,2505 & $-2,05$ & 0,2506 & $-1,98$ \\
100,00 & $5: 1$ & 0,4910 & 0,4842 & $-1,39$ & 0,4833 & $-1,59$ \\
120,00 & $6: 1$ & 0,8407 & 0,8328 & $-0,94$ & 0,8297 & $-1,31$ \\
140,00 & $7: 1$ & 1,3275 & 1,3196 & $-0,59$ & 1,3128 & $-1,10$ \\
160,00 & $8: 1$ & 1,9742 & 1,9677 & $-0,33$ & 1,9554 & $-0,95$ \\
180,00 & $9: 1$ & 2,8039 & 2,8008 & $-0,11$ & 2,7806 & $-0,83$ \\
200,00 & $10: 1$ & 3,8392 & 3,8421 & 0,07 & 3,8111 & $-0,73$ \\
\hline
\end{tabular}

Tabela 7.25 - Número de condicionamento - exemplo 3 - elemento quadrático

\begin{tabular}{|c|c|c|c|}
\hline \multirow{2}{*}{$\begin{array}{l}\text { ALTURA } \\
(\mathrm{cm})\end{array}$} & \multirow{2}{*}{$\begin{array}{c}\text { RELAÇÃO } \\
\mathrm{H}: \mathrm{B}\end{array}$} & \multicolumn{2}{|c|}{$\begin{array}{c}\text { NÚMERO DE } \\
\text { CONDICIONAMENTO }\end{array}$} \\
\hline & & ISSA & ISQG \\
\hline 20,00 & $1: 1$ & 609.728 & 3.893 .589 \\
\hline 40,00 & $2: 1$ & 702.114 & 3.417 .754 \\
\hline 60,00 & $3: 1$ & 647.384 & 3.419 .552 \\
\hline 80,00 & $4: 1$ & 509.556 & 3.365.744 \\
\hline 100,00 & $5: 1$ & 677.871 & 3.284 .033 \\
\hline 120,00 & $6: 1$ & 744.057 & 3.184 .875 \\
\hline 140,00 & $7: 1$ & 871.778 & 3.075 .808 \\
\hline 160,00 & $8: 1$ & 982.598 & 2.961 .449 \\
\hline 180,00 & $9: 1$ & 1.111 .727 & 2.845 .606 \\
\hline 200,00 & 10:1 & 1.246 .404 & 2.748 .986 \\
\hline
\end{tabular}

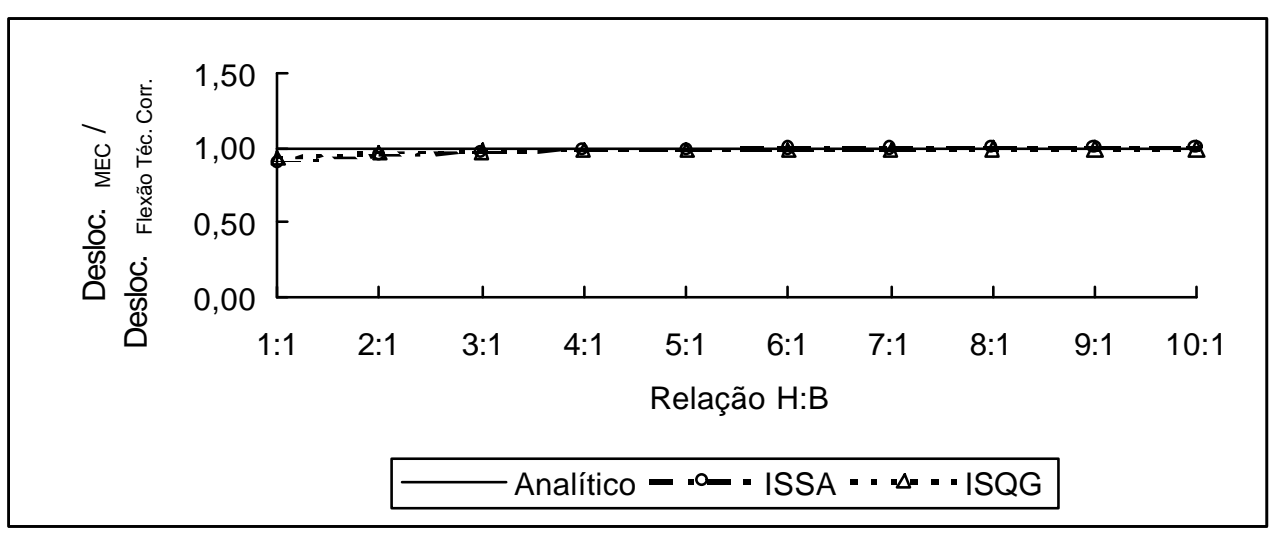

Gráfico 7.15 - Comportamento do deslocamento com a variação do comprimento elemento quadrático 
O elemento quadrático, como previsto, apresentou resultados muito bons e com pequena diferença entre as formulações. Outro ponto que se deve prestar atenção é o fato de que quando se aumenta o comprimento do sólido, o erro diminui, o que era esperado, pois a teoria de flexão técnica prevê que quanto maior o comprimento do sólido em relação à dimensões da base, maior a aproximação da teoria com a realidade. Para os sólidos com comprimentos menores do que 6 vezes a dimensão da base $(L<6 h)$, como pode ser visto em Schiel (1984), a correção dos deslocamentos devido ao cisalhamento mostra-se bastante importante, pois tal correção, nos casos especificados acima, é da mesma ordem de grandeza dos deslocamentos devido à flexão. Caso não fosse feita essa correção, o erro poderia ser até de $50 \%$. Observa-se também que para peças muito curtas, $L<3 h$, a correção efetuada já não é tão precisa, refletindo no erro aparente apresentado.

\subsection{Exemplo 4 - Viga bi-apoiada sob força transversal no meio do vão}

Neste exemplo modela-se uma viga bi-apoiada sob flexão simples devido a uma força transversal concentrada no meio do vão. As propriedades geométricas e físicas são mostradas na figura 7.8. A discretização para o elemento linear, assim como a distribuição do carregamento e apoios da viga; podem ser vistos na figura 7.9. O carregamento é distribuído de forma a simular o carregamento concentrado, gerando um volume na área destacada, assim como os apoios. Calcula-se essa viga com dois comprimentos, $80 \mathrm{~cm}(966 \mathrm{GL})$ e $160 \mathrm{~cm}(1734 \mathrm{GL})$, possuindo $70 \mathrm{~cm}$ e $150 \mathrm{~cm}$ de vãos livres, respectivamente, mantendo-se a mesma densidade de malha.

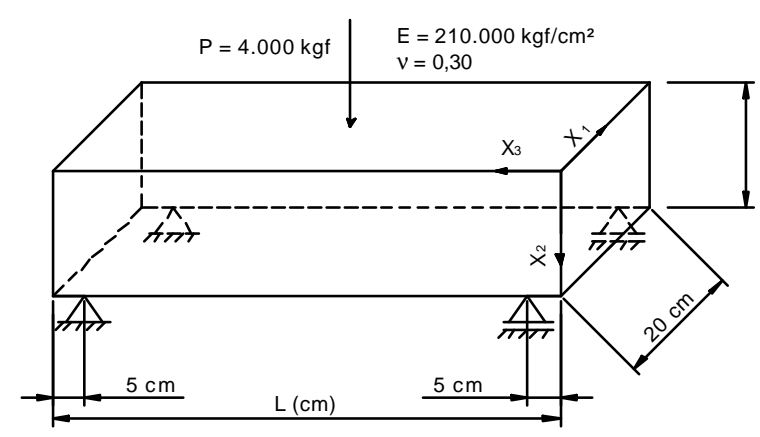

Figura 7.8 - Viga bi-apoiada com carregamento concentrado 


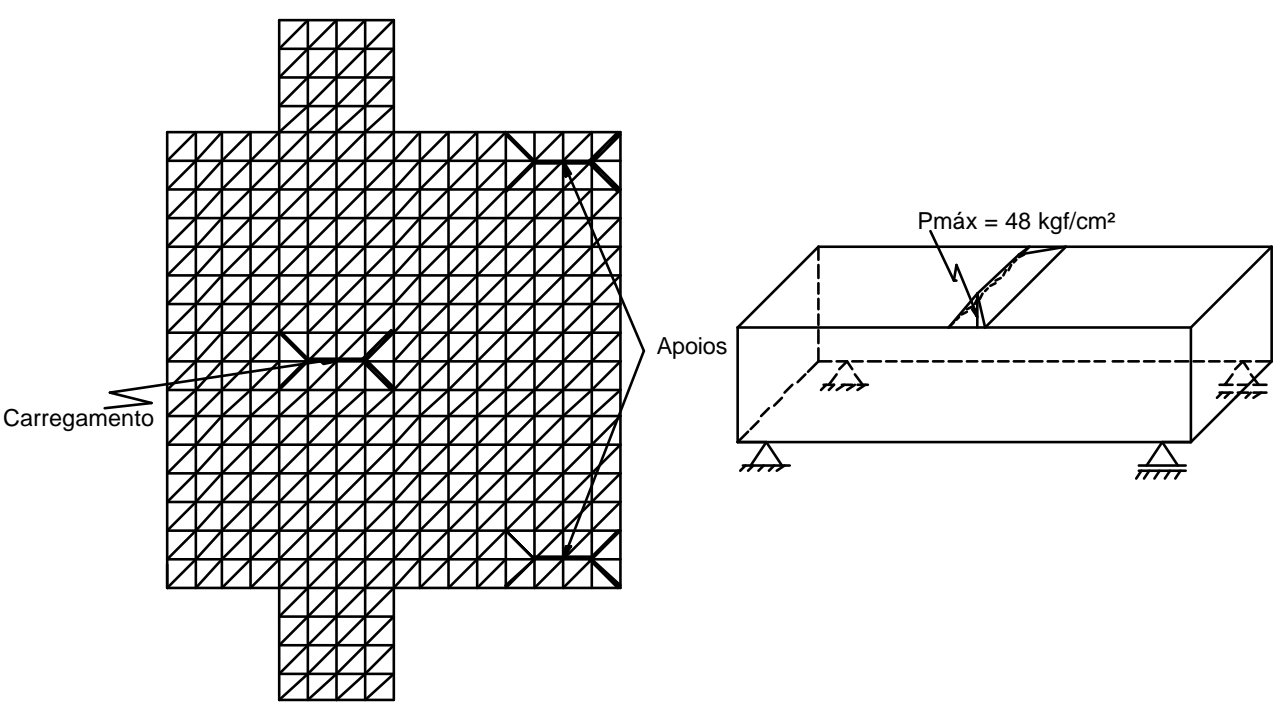

Figura 7.9 - Discretização da viga de $80 \mathrm{~cm}$ indicando carregamento e apoios para o elemento linear

Apresenta-se no gráfico 7.16 a estabilidade deste elemento com a discretização adotada para a viga de comprimento $80 \mathrm{~cm}$ e na tabela 7.26 , os resultados de deslocamentos transversais $\left(\mathrm{X}_{2}\right)$ no ponto central da viga para os dois comprimentos já mencionados, comparando-se com valores analíticos encontrados em Schiel (1984), considerando-se as deformações devido ao cisalhamento. É comparado novamente o número de condicionamento (tabela 7.27) para verificar a estabilidade do sistema linear. É utilizado o fator de 0,2 para a técnica do ponto fora, por este valor apresentar os melhores resultados no intervalo adotado, como pode ser visto no gráfico 7.16. Além disso, o nível de estabilidade está bastante razoável.

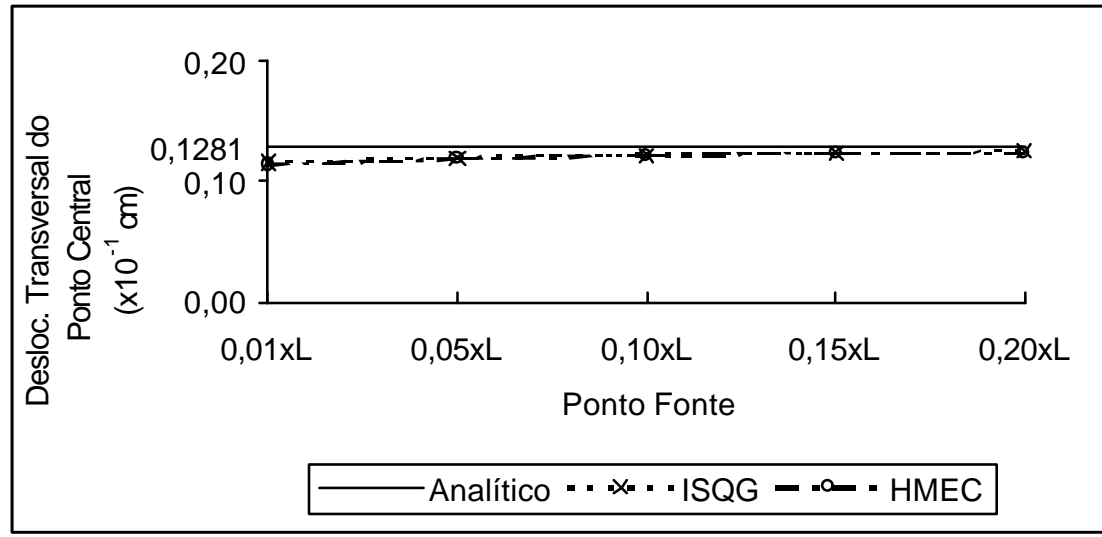

Gráfico 7.16 - Estabilidade da solução para o elemento linear - exemplo 4 
Tabela 7.26 - Deslocamentos transversais $\left(X_{2}\right)$ do exemplo 4 - elemento linear

\begin{tabular}{|c|c|c|c|c|c|c|c|}
\hline VÃO LIVRE & FLEXÃO TÉC. CORRIGIDA & & & FON & ULAÇÃO & & \\
\hline$(\mathrm{cm})$ & DESLOC. EM $X_{2}\left(\times 10^{-1} \mathrm{~cm}\right)$ & ISSA & ERRO (\%) & ISQG & ERRO (\%) & HMEC & ERRO (\%) \\
\hline 70,00 & 0,1281 & 0,1137 & $-11,21$ & $0,124 \varsigma$ & $-2,52$ & 0,1243 & $-2,93$ \\
\hline 150,00 & 1,0602 & 0,8499 & $-19,83$ & 1,0084 & $-4,88$ & 0,9829 & $-7,29$ \\
\hline
\end{tabular}

Tabela 7.27 - Número de condicionamento - exemplo 4 - elemento linear

\begin{tabular}{|c|c|c|c|c|}
\hline COMPRIM. & VÃO LIVRE & NÚMERO & CONDIC & JAMENTO \\
\hline$(\mathrm{cm})$ & $(\mathrm{cm})$ & ISSA & ISQG & HMEC \\
\hline 80,0 & $x^{2}+2$ & 67.689 & ( & 1.5 \\
\hline 160,00 & 150,00 & 84.509 & 200.449 & 139.401 \\
\hline
\end{tabular}

Com a finalidade de comparar as formulações e também os elementos utilizados apresentam-se as tabelas 7.28 e 7.29, onde são mostrados os deslocamentos transversais $\left(\mathrm{X}_{2}\right)$ para vários pontos sobre o eixo central da viga com comprimentos 80 e $160 \mathrm{~cm}$, respectivamente, e plotam-se esses valores nos gráficos 7.17 e 7.18 .

Tabela 7.28 - Deslocamentos transversais para pontos sobre o eixo central - viga $80 \mathrm{~cm}$ - elemento linear

DIST. DO APOIO FLEXÃO TÉC. CORRIG. FOMULAÇÃO (cm) DESLOC. EM X $X_{2}\left(\times 10^{-1} \mathrm{~cm}\right)$ ISSA ERRO (\%) ISQG ERRO (\%) HMEC ERRO (\%)

$\begin{array}{llllllll}25 & 0,1093 & 0,0998 & -8,74 & 0,1096 & 0,21 & 0,1091 & -0,25 \\ 35 & 0,1281 & 0,1137 & -11,21 & 0,1249 & -2,52 & 0,1243 & -2,93 \\ 45 & 0,1093 & 0,0998 & -8,74 & 0,1096 & 0,21 & 0,1091 & -0,25\end{array}$

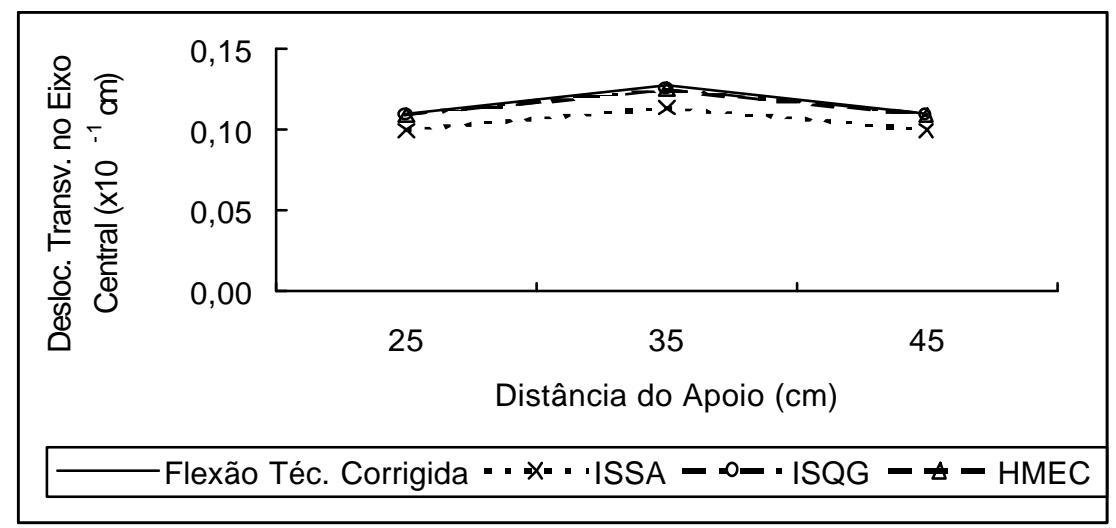

Gráfico 7.17 - Deslocamentos transversais para pontos sobre o eixo central - viga $80 \mathrm{~cm}$ - elemento linear 
Tabela 7.29 - Deslocamentos transversais para pontos sobre o eixo central - viga $160 \mathrm{~cm}$ - elemento linear

\begin{tabular}{|c|c|c|c|c|c|c|c|}
\hline \multirow{2}{*}{$\begin{array}{l}\text { DIST. DO APOIO } \\
(\mathrm{cm}) \\
\end{array}$} & \multirow{2}{*}{$\begin{array}{l}\text { FLEXÃO TÉC. CORRIG. } \\
\text { DESLOC. EM X } X_{2}\left[\times 10^{-1} \mathrm{~cm}\right]\end{array}$} & \multicolumn{6}{|c|}{ FOMULAÇÃO } \\
\hline & & ISSA & ERRO (\%) & ISQG & ERRO (\%) & HMEC & ERRO (\%) \\
\hline 35 & 0,6781 & 0,5444 & $-19,72$ & 0,6494 & $-4,24$ & 0,6342 & $-6,47$ \\
\hline 45 & 0,8290 & 0,6654 & $-19,74$ & 0,7927 & $-4,37$ & 0,7741 & $-6,62$ \\
\hline 55 & 0,9477 & 0,7609 & $-19,71$ & 0,9053 & $-4,47$ & 0,8837 & $-6,75$ \\
\hline 65 & 1,0272 & 0,8252 & $-19,66$ & 0,9802 & $-4,57$ & 0,9563 & $-6,90$ \\
\hline 75 & 1,0602 & 0,8499 & $-19,83$ & 1,0084 & $-4,88$ & 0,9829 & $-7,29$ \\
\hline 85 & 1,0272 & 0,8253 & $-19,65$ & 0,9791 & $-4,68$ & 0,9527 & $-7,25$ \\
\hline 95 & 0,9477 & 0,7611 & $-19,69$ & 0,9030 & $-4,71$ & 0,8766 & $-7,50$ \\
\hline 105 & 0,8290 & 0,6655 & $-19,72$ & 0,7894 & $-4,77$ & 0,7639 & $-7,85$ \\
\hline 115 & 0,6781 & 0,5446 & $-19,69$ & 0,6451 & $-4,86$ & 0,6213 & $-8,38$ \\
\hline
\end{tabular}

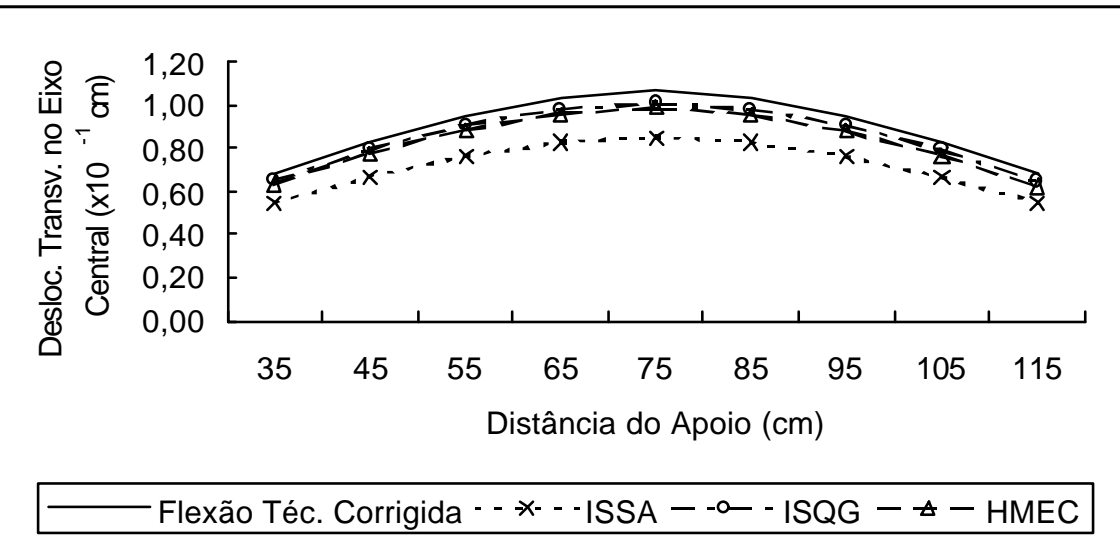

Gráfico 7.18 - Deslocamentos transversais para pontos sobre o eixo central - viga $160 \mathrm{~cm}$ - elemento linear

Os resultados obtidos com o ISQG foram um pouco melhores do que o HMEC e bem melhores do que o ISSA, que dispõe o ponto fonte no contorno, apesar do número de condicionamento desta última formulação ser menor, o que mostra que o sistema linear está consistente, mas a ordem de grandeza do número de condicionamento não interfere muito nos resultados. Como o exemplo anterior, o ponto fonte externo ao contorno conduz a melhores resultados, pois, em se tratando de um sólido retangular, as sub-matrizes da diagonal principal da matriz $\mathrm{H}$ possuem valores semelhantes aos do exemplo anterior. Além disso, para melhorar os resultados com pontos fonte mais próximos bastaria aumentar a densidade da malha, buscando uma maior estabilidade (gráfico 7.16).

Para o elemento quadrático utiliza-se a discretização para a viga de $80 \mathrm{~cm}$ e a forma de carregamento mostrados na figura 7.10 , e para a viga de $160 \mathrm{~cm}$ manteve- 
se a mesma densidade de malha. No gráfico 7.19 mostra-se a estabilidade da discretização da figura 7.10 para o elemento quadrático. Na tabela 7.30 estão os deslocamentos transversais para a viga com os comprimentos $80 \mathrm{~cm}$ (984 GL) e $160 \mathrm{~cm}$ (1752 GL) e na tabela 7.31, os números de condicionamento deste elemento para a discretização apresentada. Utilizou-se 0,1 como fator para a técnica do ponto fora.

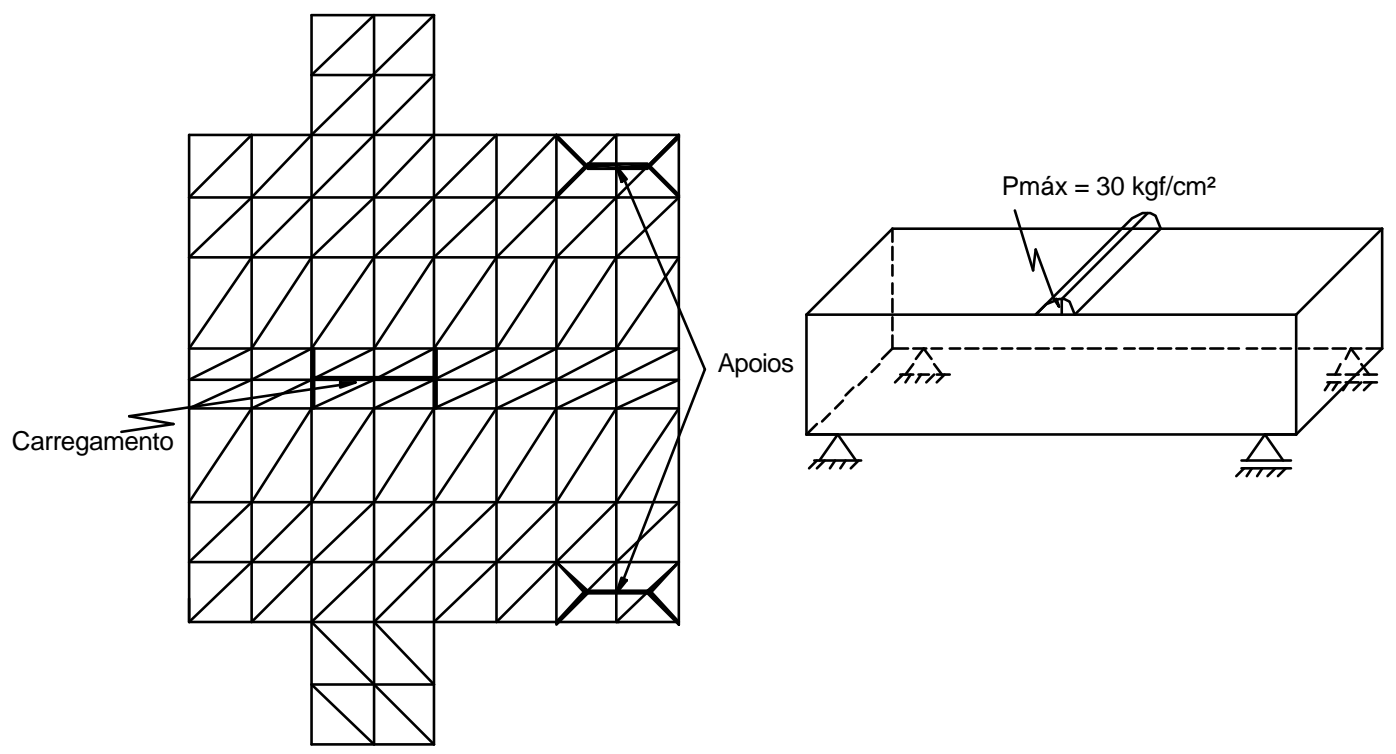

Figura 7.10 - Discretização do sólido do exemplo 4 indicando carregamento e apoios para o elemento quadrático

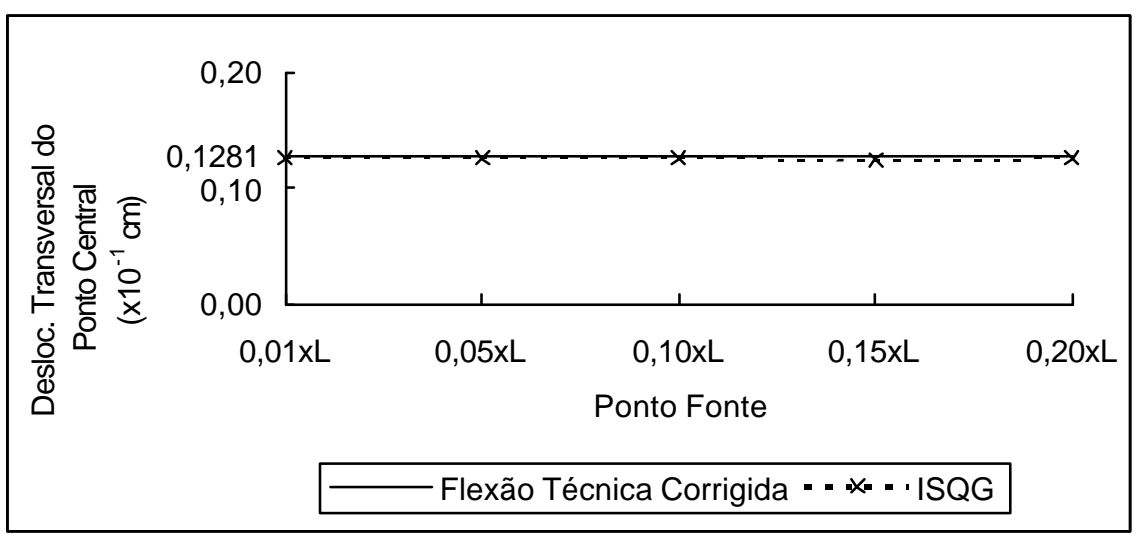

Gráfico 7.19 - Estabilidade da solução para o elemento quadrático - exemplo 4 
Tabela 7.30 - Deslocamentos transversais $\left(X_{2}\right)$ do exemplo 4 - elemento quadrático

\begin{tabular}{|c|c|c|c|c|c|c|}
\hline \multirow{2}{*}{$\begin{array}{l}\text { COMPRIM. } \\
(\mathrm{cm})\end{array}$} & \multirow{2}{*}{$\begin{array}{l}\text { VÃO LIVRE } \\
(\mathrm{cm})\end{array}$} & \multirow{2}{*}{$\begin{array}{l}\text { FLEXÃO TÉC. CORRIGIDA } \\
\text { DESLOC. EM } X_{2}\left(\times 10^{-1} \mathrm{~cm}\right)\end{array}$} & \multicolumn{4}{|c|}{ FOMULAÇÃO } \\
\hline & & & ISSA & ERRO (\%) & ISQG & ERRO (\%) \\
\hline 80,00 & 70,00 & 0,1281 & 0,1259 & $-1,73$ & 0,1265 & $-1,24$ \\
\hline 160,00 & 150,00 & 1,0602 & 1,0560 & $-0,39$ & 1,0653 & 0,48 \\
\hline
\end{tabular}

Tabela 7.31 - Número de condicionamento - exemplo 4 - elemento quadrático

\begin{tabular}{cccr}
\hline $\begin{array}{c}\text { COMPRIM. } \\
(\mathrm{cm})\end{array}$ & $\begin{array}{c}\text { VÃO LIVRE } \\
(\mathrm{cm})\end{array}$ & $\begin{array}{c}\text { NÚMERO DE CONDICIONAMENTO } \\
\text { ISSA }\end{array}$ & \multicolumn{1}{c}{ ISQG } \\
\hline & & & \\
80,00 & 70,00 & 78.798 & 5.386 .556 \\
160,00 & 150,00 & 66.344 & 731.693 \\
& & & \\
\hline
\end{tabular}

O elemento quadrático mostrou-se bastante estável com praticamente 0 mesmo número de graus de liberdade do elemento linear, e, como no exemplo 2 , os resultados com o ponto fonte no contorno (ISSA) são tão bons quanto o ponto fonte externo ao contorno (ISQG); isto se deve ao fato do elemento quadrático representar bem o problema e sua discretização ter um grau de refinamento adequado. Apresentam-se os deslocamentos transversais para pontos sobre o eixo central da viga de $80 \mathrm{~cm}$ (tabela 7.32) e da viga de $160 \mathrm{~cm}$ (tabela 7.33) e plotou-se esses resultados nos gráficos 7.20 e 7.21, respectivamente.

Tabela 7.32 - Deslocamentos transversais para pontos sobre o eixo central - viga $80 \mathrm{~cm}$ - elemento quadrático

\begin{tabular}{cccccc}
\hline $\begin{array}{c}\text { DIST. DO APOIO FLEXÃO TÉC. CORRIGIDA } \\
(\mathrm{cm})\end{array}$ & DESLOC. EM X $\mathrm{X}_{2}\left(\times 10^{-1} \mathrm{~cm}\right)$ & ISSA & ERRO (\%) & ISQG & ERRO (\%) \\
\hline & & & & & \\
25 & 0,1093 & 0,1106 & 1,12 & 0,1114 & 1,88 \\
35 & 0,1281 & 0,1259 & $-1,73$ & 0,1265 & $-1,24$ \\
45 & 0,1093 & 0,1106 & 1,12 & 0,1114 & 1,91 \\
\hline
\end{tabular}




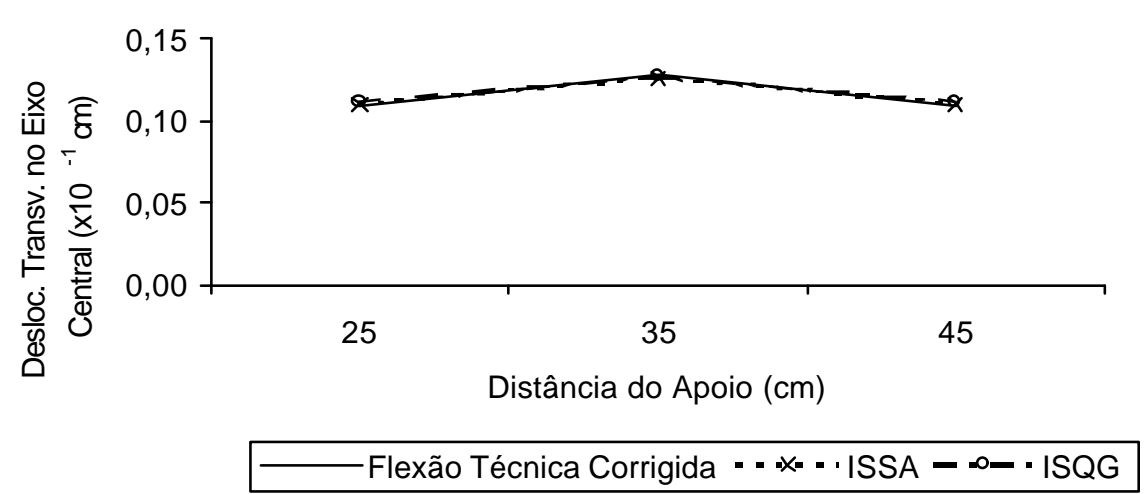

Gráfico 7.20 - Deslocamentos transversais para pontos sobre o eixo central - viga $80 \mathrm{~cm}$ - elemento quadrático

Tabela 7.33 - Deslocamentos transversais para pontos sobre o eixo central - viga $160 \mathrm{~cm}$ - elemento quadrático

\begin{tabular}{cccccc}
\hline $\begin{array}{c}\text { DIST. DO APOIO FLEXÃO TÉC. CORRIGIDA } \\
(\mathrm{cm})\end{array}$ & $\begin{array}{c}\text { FOMULACCÃO } \\
\text { DESLOC. EM } X_{2}\left(\times 10^{-1} \mathrm{~cm}\right)\end{array}$ & ISSA & ERRO $(\%)$ & ISQG & ERRO (\%) \\
\hline & & & & & \\
35 & 0,6781 & 0,6800 & 0,28 & 0,6860 & 1,16 \\
45 & 0,8290 & 0,8301 & 0,14 & 0,8375 & 1,03 \\
55 & 0,9477 & 0,9480 & 0,03 & 0,9566 & 0,94 \\
65 & 1,0272 & 1,0264 & $-0,07$ & 1,0358 & 0,84 \\
75 & 1,0602 & 1,0560 & $-0,39$ & 1,0653 & 0,48 \\
85 & 1,0272 & 1,0264 & $-0,07$ & 1,0360 & 0,86 \\
95 & 0,9477 & 0,9480 & 0,03 & 0,9568 & 0,95 \\
105 & 0,8290 & 0,8301 & 0,14 & 0,8377 & 1,05 \\
115 & 0,6781 & 0,6800 & 0,28 & 0,6862 & 1,19 \\
\hline
\end{tabular}

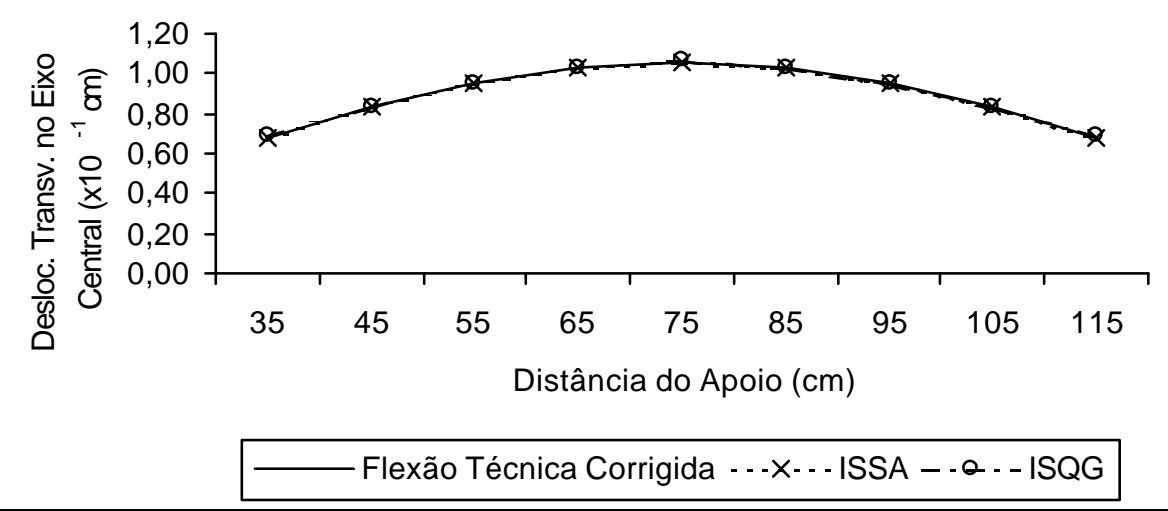

Gráfico 7.21 - Deslocamentos transversais para pontos sobre o eixo central - viga $160 \mathrm{~cm}$ - elemento quadrático 
Comparando-se os resultados obtidos entre os elementos linear e quadrático, vê-se que os resultados por este último são bem melhores e mais estáveis, justamente pela melhor representação do problema de flexão.

\subsection{Exemplo 5 - Esfera vazada sob pressão interna}

Este exemplo trata-se de uma esfera vazada ou oca (figura 7.11) submetida a uma pressão interna unitária, com raio interno unitário e raio externo com valores entre 1,01 e 2,00. Possui módulo de elasticidade unitário e coeficiente de Poisson 0,3 . Foi mantido o mesmo número de elementos para todos os valores de raios externos, com um total de 864 elementos (532 nós e 1596 graus de liberdade) para o elemento linear, como mostra o corte da figura 7.12. Esta discretização foi obtida a partir da discretização de um cubo de lado igual ao diâmetro da esfera.

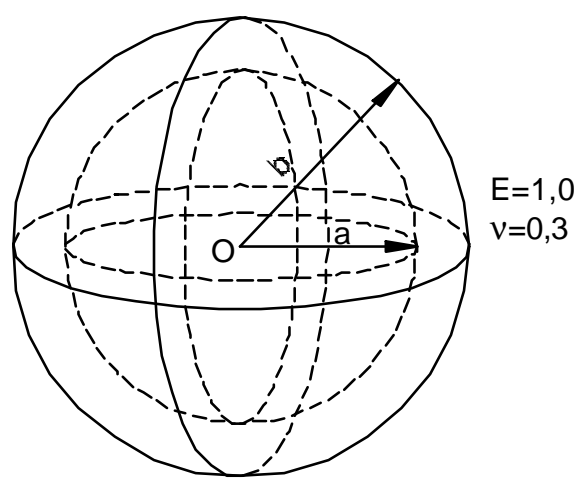

Figura 7.11 - Esfera vazada

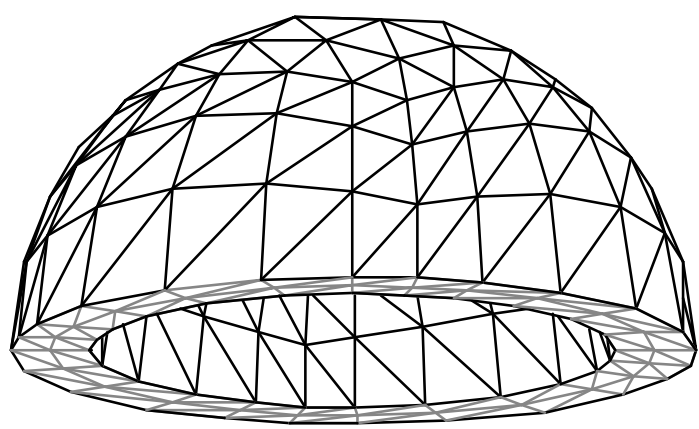

Figura 7.12 - Corte no plano médio da esfera discretizada

No gráfico 7.22 mostra-se a estabilidade do elemento linear para a esfera com relação b/a=1,01 e no gráfico 7.23 para a relação b/a=2,0. 
$\mathrm{Na}$ tabela 7.34 comparam-se os valores dos deslocamentos radiais para $r=a$ (raio interno) para várias relações b/a com valores analíticos (ver apêndice I) e no gráfico 7.24 encontram-se esses valores plotados. Na tabela 7.35 apresentam-se os valores para as tensões circunferenciais, assim como seus valores plotados no gráfico 7.25 e na tabela 7.36 os números de condicionamento. Utiliza-se 0,2 como fator para a técnica do ponto fora.

\begin{tabular}{|c|c|c|c|c|c|}
\hline \multicolumn{2}{|c|}{ DIST.DO APOOFEXÃO TÉC. CORRIGIA } & \multicolumn{4}{|c|}{ FOMULAÇÃO } \\
\hline (cm) & DESLOC. EM $X_{2}\left(x 10^{-1} \mathrm{~cm}\right)$ & ISSA & ERRO $(\%)$ & ISQG & 由RO (\%) \\
\hline 35 & 0,6781 & 0,6800 & 0,28 & 0,6860 & 1,16 \\
\hline 45 & 0,8290 & 0,8301 & 0,14 & 0,8375 & 1,03 \\
\hline 55 & 0,9477 & 0,9480 & 0,03 & 0,9566 & 0,94 \\
\hline 65 & 1,0272 & 1,0264 & $-0,07$ & 1,0358 & 0,84 \\
\hline 75 & 1,0602 & 1,0560 & $-0,39$ & 1,0653 & 0,48 \\
\hline 85 & 1,0272 & 1,0264 & $-0,07$ & 1,0360 & 0,86 \\
\hline 95 & 0,9477 & 0,9480 & 0,03 & 0,9568 & 0,95 \\
\hline 105 & 0,8290 & 0,8301 & 0,14 & 0,8377 & 1,05 \\
\hline 115 & 0,6781 & 0,6800 & 0,28 & 0,6862 & 1,19 \\
\hline
\end{tabular}

Gráfico 7.22 - Estabilidade do elemento linear para a esfera com relação b/a=1,01

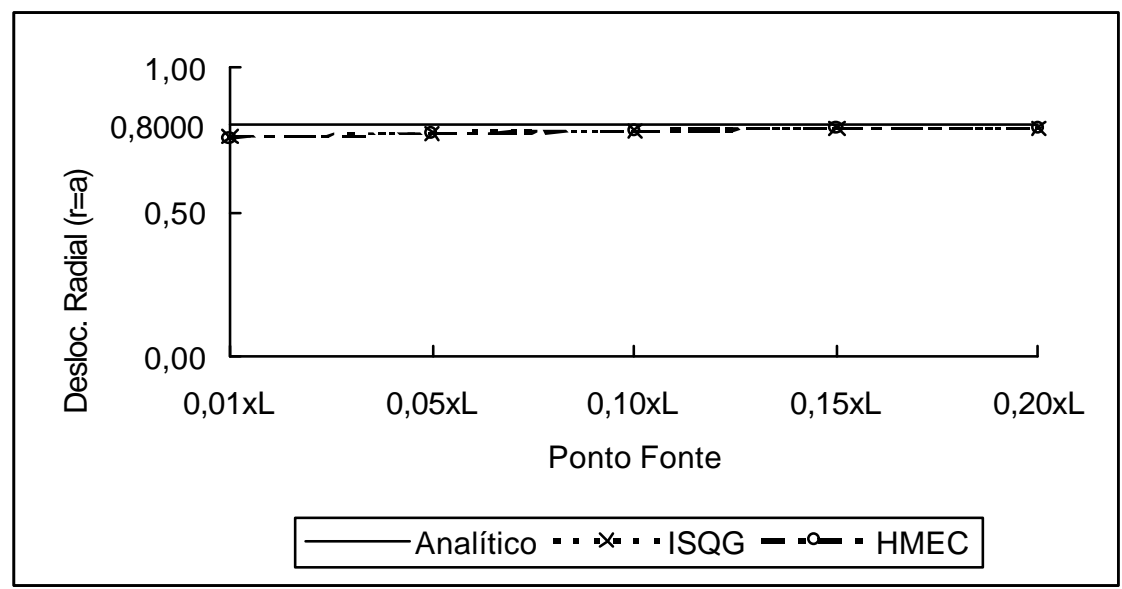

Gráfico 7.23 - Estabilidade do elemento linear para a esfera com relação b/a=2,0 
Tabela 7.34 - Deslocamentos radiais para $r=a$ - elemento linear
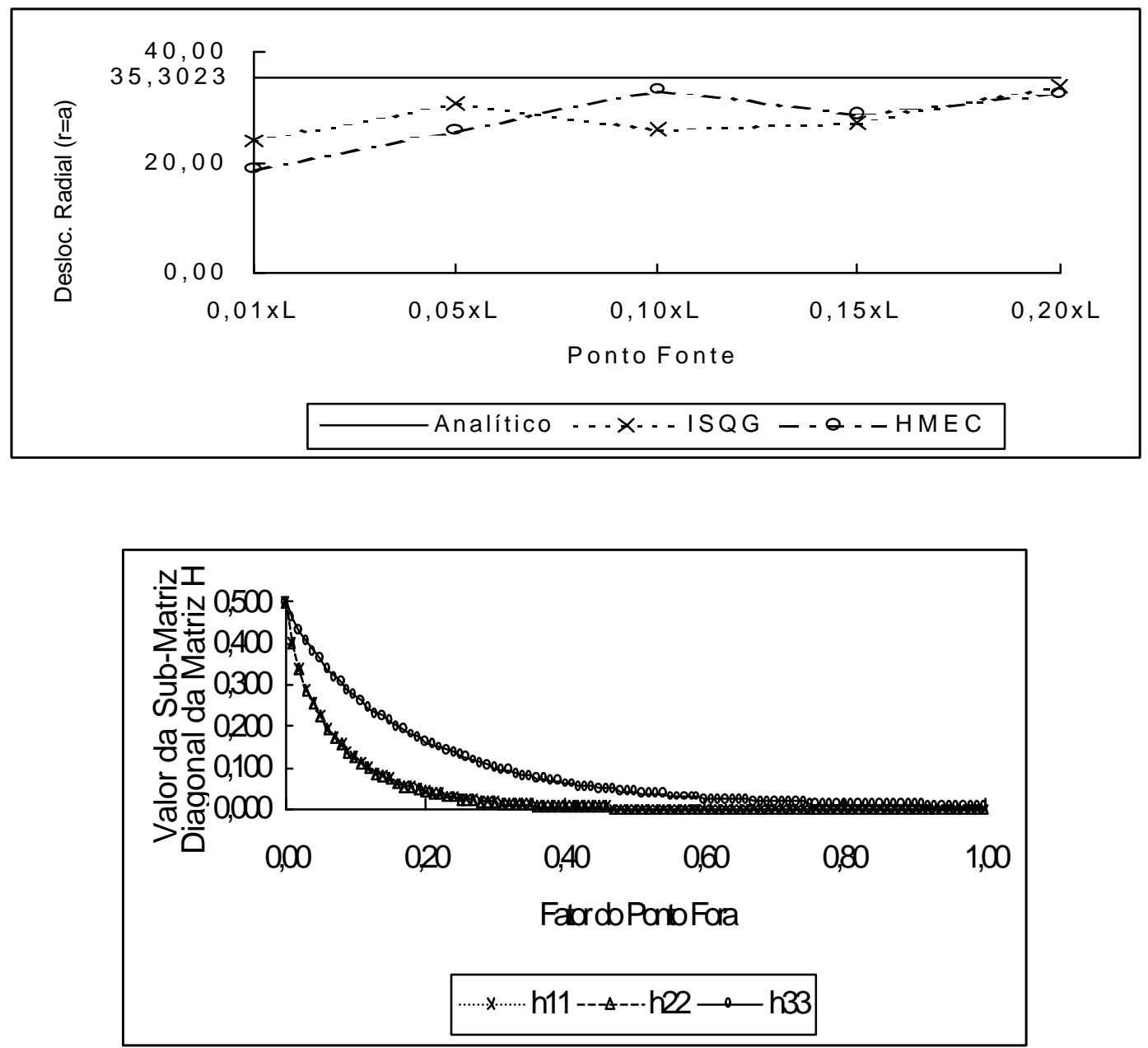

Gráfico 7.24 - Deslocamentos radiais para $r=a$ - elemento linear

Tabela 7.35 - Tensões circunferenciais para $r=a$ - elemento linear

\begin{tabular}{cccccccc}
\hline $\begin{array}{c}\text { RELAÇÃO } \\
\text { b/a }\end{array}$ & $\begin{array}{c}\text { TENSÃO CIRCUNF. } \\
(\mathrm{r}=\mathrm{a})\end{array}$ & ISSA & ERRO (\%) & $\begin{array}{c}\text { FORMULAÇÃO } \\
\text { ISQG }\end{array}$ & ERRO (\%) & HMEC & ERRO (\%) \\
\hline & & & & & & & \\
1,01 & 50,0033 & 31,3070 & $-37,39$ & 47,3680 & $-5,27$ & 46,8730 & $-6,26$ \\
1,02 & 25,0066 & 17,1080 & $-31,59$ & 23,7500 & $-5,03$ & 23,5570 & $-5,80$ \\
1,03 & 16,6765 & 12,0190 & $-27,93$ & 15,9060 & $-4,62$ & 15,8060 & $-5,22$ \\
1,04 & 12,5131 & 9,3514 & $-25,27$ & 11,9390 & $-4,59$ & 11,8940 & $-4,95$ \\
1,05 & 10,0163 & 7,6938 & $-23,19$ & 9,5650 & $-4,51$ & 9,5410 & $-4,74$ \\
1,10 & 5,0317 & 4,1750 & $-17,03$ & 4,8566 & $-3,48$ & 4,8863 & $-2,89$ \\
1,20 & 2,5604 & 2,2464 & $-12,27$ & 2,5843 & 0,93 & 2,6141 & 2,10 \\
1,40 & 1,3601 & 1,2325 & $-9,38$ & 1,3519 & $-0,60$ & 1,3593 & $-0,06$ \\
1,60 & 0,9845 & 0,9048 & $-8,10$ & 0,9972 & 1,29 & 1,0000 & 1,57 \\
1,80 & 0,8104 & 0,7517 & $-7,24$ & 0,8328 & 2,76 & 0,8341 & 2,92 \\
2,00 & 0,7143 & 0,6670 & $-6,63$ & 0,7419 & 3,87 & 0,7426 & 3,97 \\
& & & & & & & \\
\hline
\end{tabular}




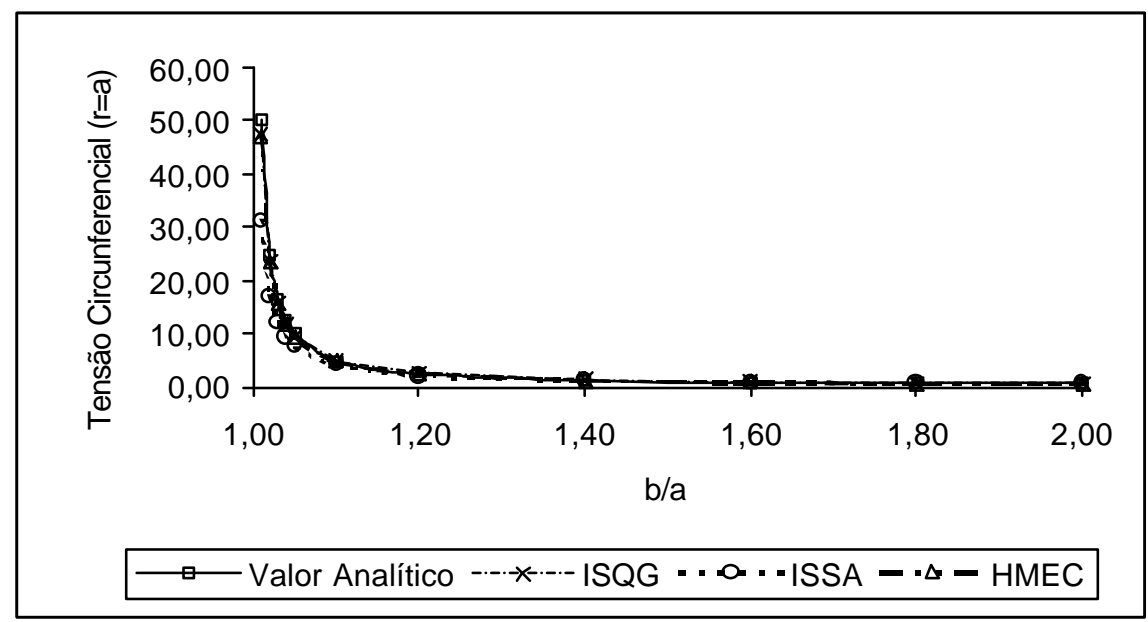

Gráfico 7.25 - Tensões circunferenciais para $r=a$ - elemento linear

Tabela 7.36 - Número de condicionamento - exemplo 5 - elemento linear

\begin{tabular}{|c|c|c|c|}
\hline \multirow{2}{*}{$\begin{array}{c}\text { RELAÇÃO } \\
\text { b/a }\end{array}$} & \multicolumn{3}{|c|}{ NÚMERO DE CONDICIONAMENTO } \\
\hline & ISSA & ISQG & HMEC \\
\hline 1,01 & 4.788 & 493.421 & 544.109 \\
\hline 1,02 & 2.799 & 1.123 .002 & 1.753 .326 \\
\hline 1,03 & 2.152 & 834.054 & 1.111 .747 \\
\hline 1,04 & 1.810 & 339.687 & 352.303 \\
\hline 1,05 & 1.618 & 1.577 .043 & 3.624 .248 \\
\hline 1,10 & 1.257 & 129.549 & 281.498 \\
\hline 1,20 & 1.204 & 308.770 & 352.614 \\
\hline 1,40 & 1.391 & 71.466 & 83.819 \\
\hline 1,60 & 1.592 & 64.412 & 94.812 \\
\hline 1,80 & 1.793 & 56.482 & 84.737 \\
\hline 2,00 & 2.000 & 53.095 & 92.508 \\
\hline
\end{tabular}

O elemento linear não se mostrou estável para a relação $b / a=1,01$, mas conduz a bons resultados quando se utiliza o fator 0,2 . Mas àmedida que a relação b/a vai aumentando, a estabilidade também aumenta. Novamente cs melhores resultados são obtidos com a utilização da formulação com o ponto fonte fora do contorno (ISQG), seguida pela formulação que utiliza as funções hiper-singulares (HMEC), que se mostrou muito boa para este tipo de problema. Os números de condicionamento da formulação com o ponto fonte no contorno (ISSA) foram bem menores do que as outras duas formulações, mostrando que o sistema está bastante estável para esta formulação, apesar disso os resultados não foram bons. Dessa forma apresenta-se no gráfico 7.26 um estudo da sub-matriz $(3 \times 3)$ da 
diagonal principal da matriz $\mathrm{H}$ para o ponto central superior, como feito analogamente para outros exemplos.
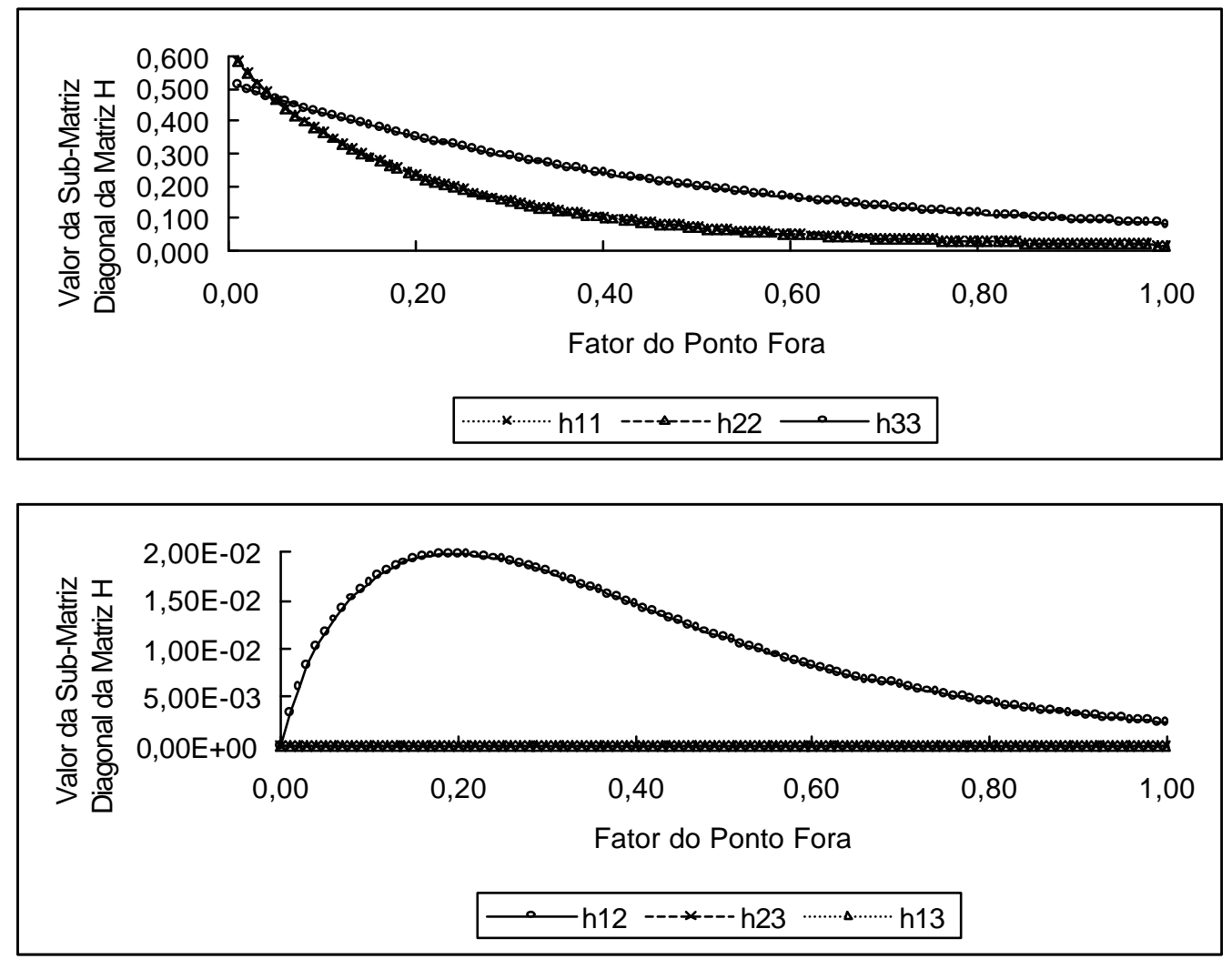

\section{Gráfico 7.26 - Sub-matriz da diagonal principal da matriz H - elemento linear - exemplo 5}

Como nos exemplos anteriores, com o ponto fonte externo ao contorno existe um elemento da sub-matriz que é diferente de zero $\left(h_{12}\right.$ e $\left.h_{21}\right)$, além dos elementos da diagonal desta sub-matriz. Diferentemente do que acontece quando o ponto fonte está no contorno, em que apenas os elementos da diagonal desta sub-matriz são diferentes de zero. Isto melhora a solução do sistema linear, pois existem mais valores diferentes de zero. Porém, deve-se lembrar que a melhora da discretização levará a soluções estáveis e, portanto, boas para a formulação ISSA ou ISQG.

Neste exemplo não se utilizará o elemento quadrático porque para este representar bem a superfície esférica do problema precisaria de uma discretização no mínimo igual àquela utilizada para o elemento linear, pois o elemento utilizado é plano, o que aumentaria em muito o número de graus de liberdade. Além disso, o elemento linear já conduz a resultados conclusivos. 


\subsection{Exemplo 6 - Casca esférica sob pressão interna}

Este exemplo é uma pequena variação do exemplo anterior, agora é calculada a metade da esfera (casca) submetida à pressão interna, com toda a base apoiada, como mostra a figura 7.13. Este exemplo serve para analisar a perturbação causada pelas restrições devido aos apoios. A discretização é a mesma apresentada na figura 7.12, diferenciando-se a base, que agora precisa ser discretizada. Para as relações $b / a<1,10$, a base é discretizada com apenas uma fileira, por ser fina; e já para relações $b / a \geq 1,10$, a base é discretizada em duas fileiras de elementos, por essas bases já serem mais espessas. Com isso as discretizações para relações $b / a<1,10$ possuem 480 elementos e 338 nós (1014 GL) e 528 elementos e 362 nós (1086 GL) para as outras relações.

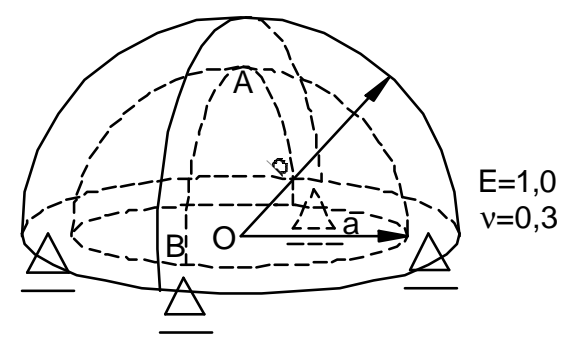

Figura 7.13 - Casca esférica

Para o cálculo dos deslocamentos escolheu-se dois pontos no contorno, um na parte central superior, Ponto A (tabelas 7.37 e 7.39), e outro na base, Ponto B (tabelas 7.38 e 7.40) para verificar a perturbação causada pelos apoios.

Tabela 7.37 - Deslocamentos radiais para $r=a$ - Ponto A - elemento linear

\begin{tabular}{cccccccc}
\hline $\begin{array}{c}\text { RELAÇÃO } \\
\text { b/a }\end{array}$ & $\begin{array}{c}\text { DESLOC. RADIAL } \\
\text { ANALIITCO }(\mathrm{r}=\mathrm{a})\end{array}$ & ISSA & ERRO (\%) & ISQG & ERRO (\%) & HMEC & ERRO (\%) \\
\hline & & & & & & & \\
1,01 & 35,3023 & 31,0330 & $-12,09$ & 38,5590 & 9,23 & 37,7180 & 6,84 \\
1,02 & 17,8046 & 16,1010 & $-9,57$ & 19,1510 & 7,56 & 18,9460 & 6,41 \\
1,03 & 11,9736 & 10,9550 & $-8,51$ & 12,7260 & 6,28 & 12,6290 & 5,47 \\
1,04 & 9,0591 & 8,3474 & $-7,86$ & 9,5051 & 4,92 & 9,4704 & 4,54 \\
1,05 & 7,3114 & 6,7726 & $-7,37$ & 7,7365 & 5,81 & 7,7933 & 6,59 \\
1,10 & 3,8222 & 3,6133 & $-5,47$ & 4,0073 & 4,84 & 4,0048 & 4,78 \\
1,20 & 2,0923 & 2,0153 & $-3,68$ & 2,1884 & 4,59 & 2,2108 & 5,66 \\
1,40 & 1,2521 & 1,2127 & $-3,14$ & 1,2681 & 1,28 & 1,2698 & 1,42 \\
1,60 & 0,9891 & 0,9521 & $-3,75$ & 0,9898 & 0,07 & 0,9839 & $-0,53$ \\
1,80 & 0,8673 & 0,8286 & $-4,46$ & 0,8620 & $-0,61$ & 0,8468 & $-2,36$ \\
2,00 & 0,8000 & 0,7589 & $-5,14$ & 0,7855 & $-1,82$ & 0,7663 & $-4,22$ \\
\hline
\end{tabular}


Tabela 7.38 - Deslocamentos radiais para $r=a$ - Ponto B - elemento linear

\begin{tabular}{cccccccc}
\hline $\begin{array}{c}\text { RELAÇÃO } \\
\text { b/a }\end{array}$ & DESLOC. RADIAL & \multicolumn{5}{c}{ FORMULAÇÃO } \\
\hline & ANALITICO $(\mathrm{r}=\mathrm{a})$ & ISSA & ERRO (\%) & ISQG & ERRO (\%) & HMEC & ERRO (\%) \\
1,01 & 35,3023 & 18,9240 & $-46,39$ & 11,0680 & $-68,65$ & 19,9060 & $-43,61$ \\
1,02 & 17,8046 & 10,7480 & $-39,63$ & 8,6331 & $-51,51$ & 11,2500 & $-36,81$ \\
1,03 & 11,9736 & 7,7602 & $-35,19$ & 7,3100 & $-38,95$ & 8,5832 & $-28,32$ \\
1,04 & 9,0591 & 6,1717 & $-31,87$ & 6,2748 & $-30,74$ & 7,0758 & $-21,89$ \\
1,05 & 7,3114 & 5,1736 & $-29,24$ & 5,2777 & $-27,82$ & 6,1821 & $-15,45$ \\
1,10 & 3,8222 & 3,0129 & $-21,17$ & 3,3200 & $-13,14$ & 3,5007 & $-8,41$ \\
1,20 & 2,0923 & 1,8032 & $-13,82$ & 2,0544 & $-1,81$ & 1,6929 & $-19,09$ \\
1,40 & 1,2521 & 1,1449 & $-8,56$ & 1,2311 & $-1,67$ & 1,2838 & 2,53 \\
1,60 & 0,9891 & 0,9233 & $-6,66$ & 0,9717 & $-1,77$ & 1,0387 & 5,01 \\
1,80 & 0,8673 & 0,8177 & $-5,72$ & 0,8516 & $-1,81$ & 0,9504 & 9,58 \\
2,00 & 0,8000 & 0,7585 & $-5,18$ & 0,7855 & $-1,82$ & 0,9311 & 16,39 \\
& & & & & & & \\
\hline
\end{tabular}

Tabela 7.39 - Tensões circunferenciais para $r=a$ - Ponto A - elemento linear

\begin{tabular}{cccccccc}
\hline $\begin{array}{c}\text { RELAÇÃO } \\
\text { b/a }\end{array}$ & TENSÃO CIRCUNF. & \multicolumn{7}{c}{ FORMULAÇÃO } \\
\hline & $(\mathrm{r}=\mathrm{a})$ & ISSA & ERRO (\%) & ISQG & ERRO (\%) & HMEC & ERRO (\%) \\
1,01 & 50,0033 & 35,4930 & $-29,02$ & 48,0840 & $-3,84$ & 48,0920 & $-3,82$ \\
1,02 & 25,0066 & 18,9540 & $-24,20$ & 24,0390 & $-3,87$ & 24,0680 & $-3,75$ \\
1,03 & 16,6765 & 13,1200 & $-21,33$ & 16,0600 & $-3,70$ & 16,0710 & $-3,63$ \\
1,04 & 12,5131 & 10,1010 & $-19,28$ & 12,0380 & $-3,80$ & 12,0590 & $-3,63$ \\
1,05 & 10,0163 & 8,2434 & $-17,70$ & 9,8041 & $-2,12$ & 9,8689 & $-1,47$ \\
1,10 & 5,0317 & 4,3734 & $-13,08$ & 4,8849 & $-2,92$ & 4,8873 & $-2,87$ \\
1,20 & 2,5604 & 2,3105 & $-9,76$ & 2,6197 & 2,31 & 2,6907 & 5,09 \\
1,40 & 1,3601 & 1,2515 & $-7,98$ & 1,3599 & $-0,01$ & 1,3371 & $-1,69$ \\
1,60 & 0,9845 & 0,9165 & $-6,90$ & 1,0023 & 1,81 & 0,9686 & $-1,62$ \\
1,80 & 0,8104 & 0,7630 & $-5,86$ & 0,8368 & 3,26 & 0,7898 & $-2,54$ \\
2,00 & 0,7143 & 0,6794 & $-4,88$ & 0,7453 & 4,34 & 0,6790 & $-4,94$ \\
& & & & & & & \\
\hline
\end{tabular}

Tabela 7.40 - Tensões circunferenciais para $r=a$ - Ponto B - elemento linear

\begin{tabular}{|c|c|c|c|c|c|c|c|}
\hline \multirow{2}{*}{$\begin{array}{c}\text { RELAÇÃO } \\
\text { b/a } \\
\end{array}$} & \multirow{2}{*}{$\begin{array}{c}\text { TENSÃO CIRCUNF. } \\
(r=a)\end{array}$} & \multicolumn{6}{|c|}{ FORMULAÇÃO } \\
\hline & & ISSA & $\mathrm{ERRO}(\%)$ & ISQG & ERRO (\%) & HMEC & ERRO (\%) \\
\hline 1,01 & 50,0033 & 38,6580 & $-22,69$ & 43,7900 & $-12,43$ & 45,1780 & $-9,65$ \\
\hline 1,02 & 25,0066 & 20,4660 & $-18,16$ & 21,9740 & $-12,13$ & 22,4140 & $-10,37$ \\
\hline 1,03 & 16,6765 & 14,0540 & $-15,73$ & 14,6780 & $-11,98$ & 15,0130 & $-9,98$ \\
\hline 1,04 & 12,5131 & 10,7510 & $-14,08$ & 11,0550 & $-11,65$ & 11,3430 & $-9,35$ \\
\hline 1,05 & 10,0163 & 8,7304 & $-12,84$ & 8,9151 & $-10,99$ & 9,1370 & $-8,78$ \\
\hline 1,10 & 5,0317 & 4,5797 & $-8,98$ & 4,3656 & $-13,24$ & 4,5595 & $-9,38$ \\
\hline 1,20 & 2,5604 & 2,4017 & $-6,20$ & 2,3473 & $-8,32$ & 1,7070 & $-33,33$ \\
\hline 1,40 & 1,3601 & 1,2912 & $-5,07$ & 1,2303 & $-9,54$ & 1,4697 & 8,06 \\
\hline 1,60 & 0,9845 & 0,9320 & $-5,34$ & 0,8935 & $-9,25$ & 1,2686 & 28,86 \\
\hline 1,80 & 0,8104 & 0,7594 & $-6,30$ & 0,7317 & $-9,72$ & 1,3114 & 61,82 \\
\hline 2,00 & 0,7143 & 0,6587 & $-7,78$ & 0,6381 & $-10,67$ & 1,5065 & 110,91 \\
\hline
\end{tabular}

E os números de condicionamento são apresentados na tabela 7.41. 
Tabela 7.41 - Número de condicionamento - exemplo 6 - elemento linear

\begin{tabular}{crrr}
\hline $\begin{array}{c}\text { RELAÇÃO } \\
\text { b/a }\end{array}$ & \multicolumn{3}{c}{ NÚMERO DE CONDICIONAMENTO } \\
ISSA & \multicolumn{1}{c}{ ISQG } & \multicolumn{1}{c}{ HMEC } \\
\hline & & & \\
1,01 & 4.706 & 2.572 .163 & 307.557 \\
1,02 & 2.446 & 7.911 .922 & 3.745 .289 \\
1,03 & 1.721 & 1.645 .726 & 3.022 .989 \\
1,04 & 964 & 105.638 & 603.666 \\
1,05 & 698 & 1.148 .200 & 2.139 .514 \\
1,10 & 778 & 197.562 & 116.714 \\
1,20 & 867 & 191.785 & 222.322 \\
1,40 & 921 & 31.987 & 31.314 \\
1,60 & 1.041 & 30.109 & 33.715 \\
1,80 & 765 & 39.146 & 38.397 \\
2,00 & 907 & 64.672 & 61.986 \\
& & & \\
\hline
\end{tabular}

Como no ponto A não há perturbação, os resultados para deslocamentos e tensões não foram muito afetados, e no ponto B é possível ver claramente que os resultados, em geral, pioraram bastante. Isto é devido à perturbação causada pelo tipo de restrição, mostrando que o MEC é muito sensível ao tipo de restrição aplicado. No caso da esfera inteira, é possível aplicar restrições para que todas as reações sejam nulas, mas no caso da casca isto não é possível. Não foi feita uma análise de estabilidade da solução para saber se esta discretização é aceitável, apenas se fez uma analogia com o problema anterior.

\subsection{Exemplo 7 - Placa retangular com flexão pura}

Este exemplo mostra a aplicação do MEC-3D em placas com curvaturas cilíndricas, esféricas e com momentos aplicados em apenas dois lados. Solucionam-se placas com várias relações $\mathrm{h} / \mathrm{L}$. Os resultados analíticos foram obtidos a partir de Timoshenko \& Woinovsky-Krieger (1956).

\subsubsection{Placa retangular com curvatura cilíndrica}

A figura 7.14 mostra a placa com curvatura cilíndrica, assim como suas propriedades físicas e geométricas, e sua discretização na figura 7.15. 


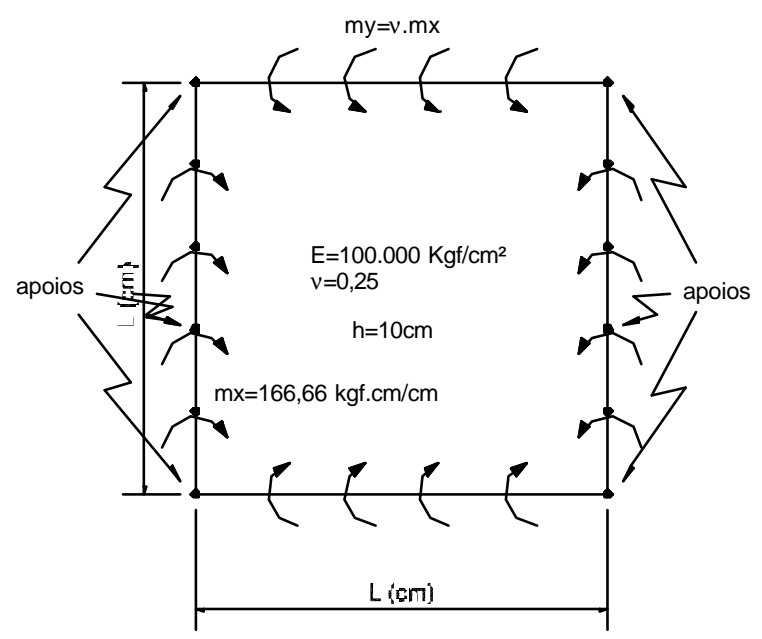

Figura 7.14 - Placa retangular com curvatura cilíndrica - elemento linear

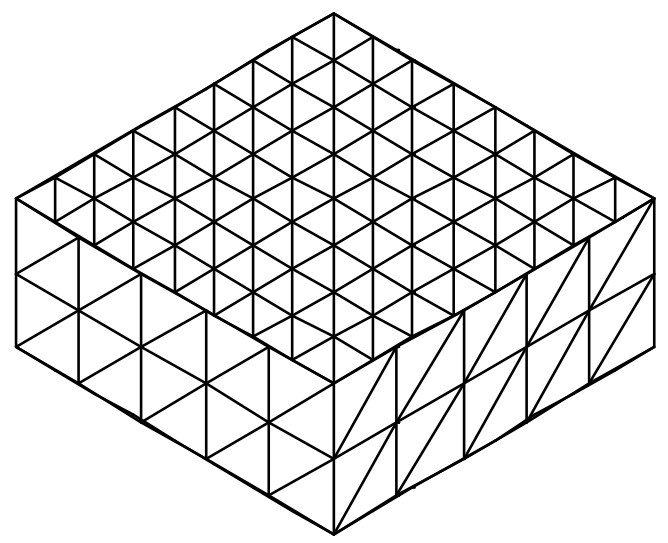

Figura 7.15 - Discretização da placa retangular - L=25cm - malha $8 \times 8$ - elemento linear

Para as placas com outras dimensões foram mantidas as mesmas densidades nas bordas e as malhas das faces, superior e inferior, ficaram as seguintes:

$\mathrm{L}=25 \mathrm{~cm}$ - malha $8 \times 8-702 \mathrm{GL}$

$\mathrm{L}=50 \mathrm{~cm}-$ malha $16 \times 16-2130 \mathrm{GL}$

$\mathrm{L}=100 \mathrm{~cm}$ - malha $24 \times 24-4506 \mathrm{GL}$

No gráfico 7.27 é mostrada a convergência para a placa retangular com $\mathrm{L}=25 \mathrm{~cm}$ e com curvatura cilíndrica. Não se mostrará a convergência para as outras placas, pois a densidade de malha foi mantida, a não ser para a placa com $\mathrm{L}=100 \mathrm{~cm}$. 


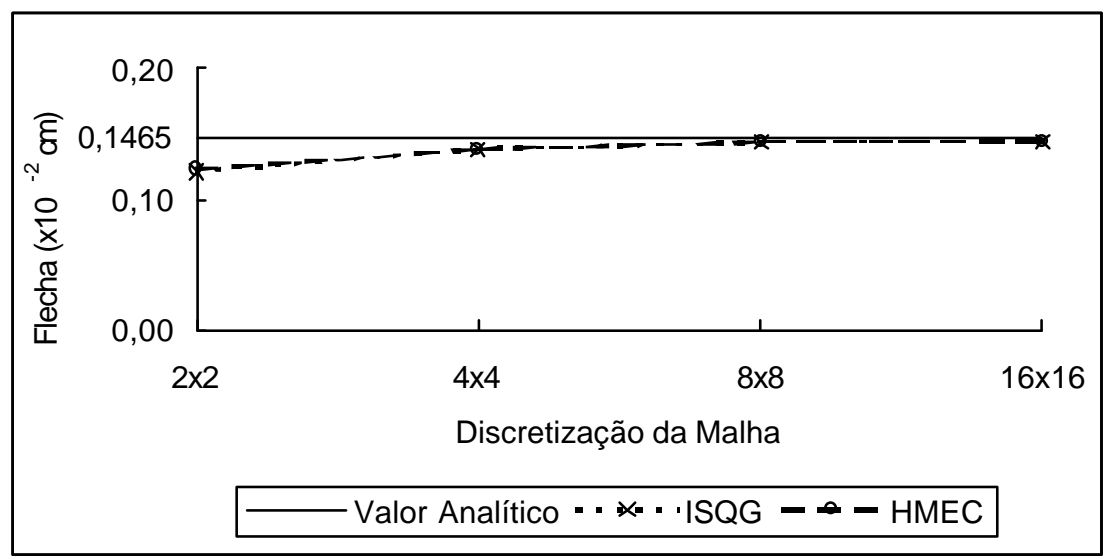

Gráfico 7.27 - Convergência da placa retangular com curvatura cilíndrica - $L=25 \mathrm{~cm}$ elemento linear

Através do gráfico 7.27 escolheu-se a malha $8 \times 8$ para se realizar a análise com o elemento linear. No gráfico 7.28 mostra-se a estabilidade desta malha para 0 elemento linear.

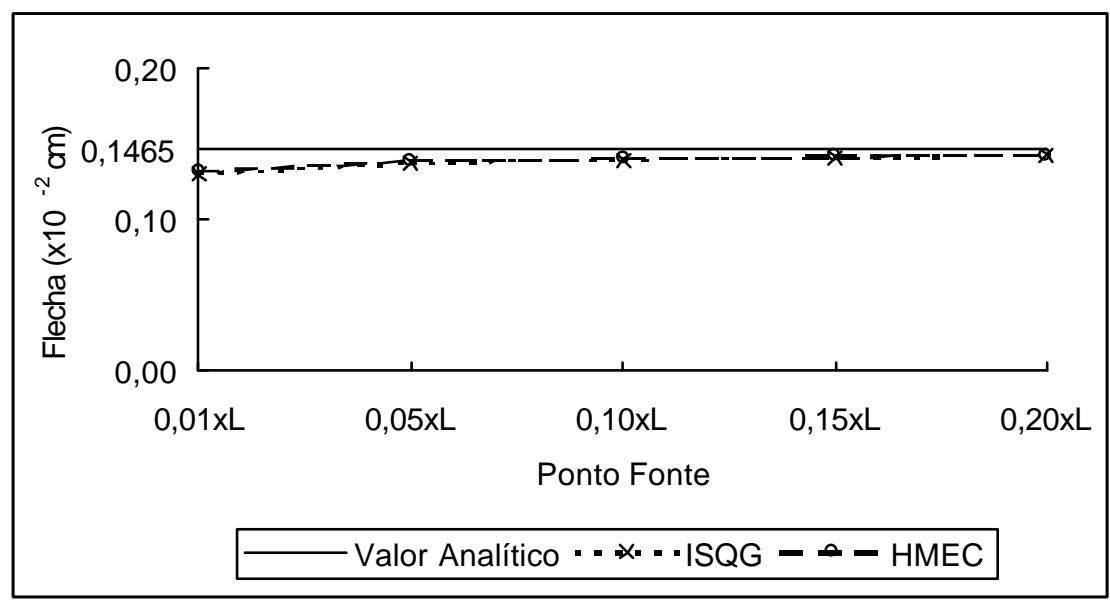

Gráfico 7.28 - Estabilidade da malha $8 \times 8-L=25 \mathrm{~cm}$ - elemento linear

A estabilidade da malha $8 \times 8$ para a placa com $L=25 \mathrm{~cm}$, ainda mostra uma pequena variação, tanto para a formulação ISQG quanto para a HMEC, mesmo assim, compara-se os deslocamentos transversais no centro da placa para os diversos comprimentos e as diversas formulações, a saber, ISSA, ISQG e HMEC para o elemento linear. Valores estes apresentados na tabela 7.42 e na tabela 7.43 apresenta-se os números de condicionamento. Utilizou o valor de 0,2 para o fator da técnica do ponto fora, por este apresentar o menor erro, segundo o gráfico 7.28. 
Tabela 7.42 - Deslocamentos em placas retangulares com curvatura cilíndrica elemento linear

\begin{tabular}{|c|c|c|c|c|c|c|c|c|}
\hline \multirow{2}{*}{$\begin{array}{c}\mathrm{L} \\
(\mathrm{cm})\end{array}$} & \multicolumn{2}{|c|}{ RELACÃO DESLOC. ANALÍTICO } & \multicolumn{6}{|c|}{ FORMULAÇÃO } \\
\hline & $H: L$ & $\operatorname{EM} X_{3}\left(x 10^{-2} \mathrm{~cm}\right)$ & ISSA & $\operatorname{ERRO}(\%)$ & ISQG & ERRO (\%) & HMEC & ERRO (\%) \\
\hline & $1:$ & & & & & $-2,58$ & & $-2,58$ \\
\hline 50 & $1:$ & 0 & 8 & -1 & 0,5 & $-2,62$ & 0, & $-2,62$ \\
\hline 100,00 & $1: 10$ & 2,3437 & 1,8129 & $-22,65$ & 2,2019 & $-6,05$ & 2,2011 & $-6,09$ \\
\hline
\end{tabular}

Tabela 7.43 - Número de condicionamento - curvatura cilíndrica - elemento linear

\begin{tabular}{rcrrr}
\hline \multicolumn{1}{c}{$\mathrm{L}$} & RELAÇÃO & \multicolumn{3}{c}{ NÚMERO DE CONDICIONAMENTO } \\
$(\mathrm{cm})$ & $\mathrm{H}: \mathrm{L}$ & ISSA & ISQG & HMEC \\
\hline & & & & \\
25,00 & $1: 2,5$ & 77.491 & 1.270 .000 & 443.569 \\
50,00 & $1: 5$ & 88.237 & 1.748 .465 & 642.178 \\
100,00 & $1: 10$ & 60.327 & 1.440 .471 & 500.840
\end{tabular}

Para as placas retangulares com curvaturas cilíndricas e resolvidas com elemento linear, o erro apresentado pela formulação ISSA (ponto fonte no contorno) foi bem maior do que as outras duas formulações, apesar do número de condicionamento ser menor. Mas como discutido anteriormente, sendo a resposta analítica uma função do $2^{0}$ grau, para que o elemento linear apresentasse resultados melhores seria necessária uma discretização maior ainda, o que acarretaria em um enorme tempo de processamento.

Para o elemento quadrático foram escolhidas as seguintes discretizações:

$\mathrm{L}=25 \mathrm{~cm}$ - malha $2 \times 2-330 \mathrm{GL}$

$\mathrm{L}=50 \mathrm{~cm}-$ malha $2 \times 2-330 \mathrm{GL}$

$\mathrm{L}=100 \mathrm{~cm}$ - malha $4 \times 4-810 \mathrm{GL}$

A discretização da malha $2 \times 2$ e $L=25 \mathrm{~cm}$ pode ser vista na figura 7.16.

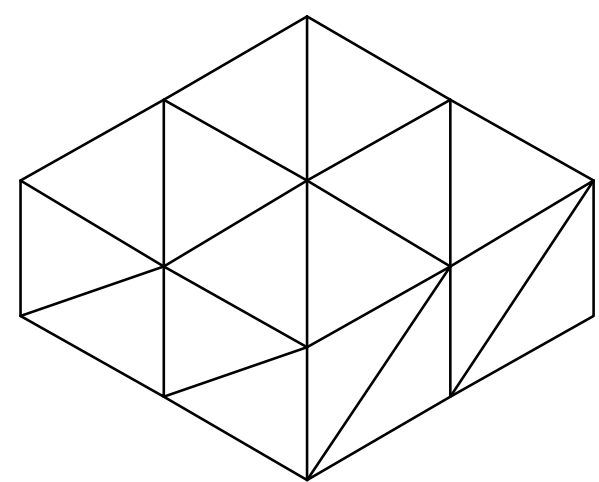

Figura 7.16 - Discretização da placa retangular - $L=25 \mathrm{~cm}$ - malha $2 \times 2$ - elemento quadrático 
O gráfico 7.29 mostra a estabilidade da placa com $L=25 \mathrm{~cm}$ e malha $2 \times 2$ para o elemento quadrático. Após isso, mostram-se os deslocamentos transversais no centro da placa na tabela 7.44 e na tabela 7.45 são apresentados os números de condicionamento.

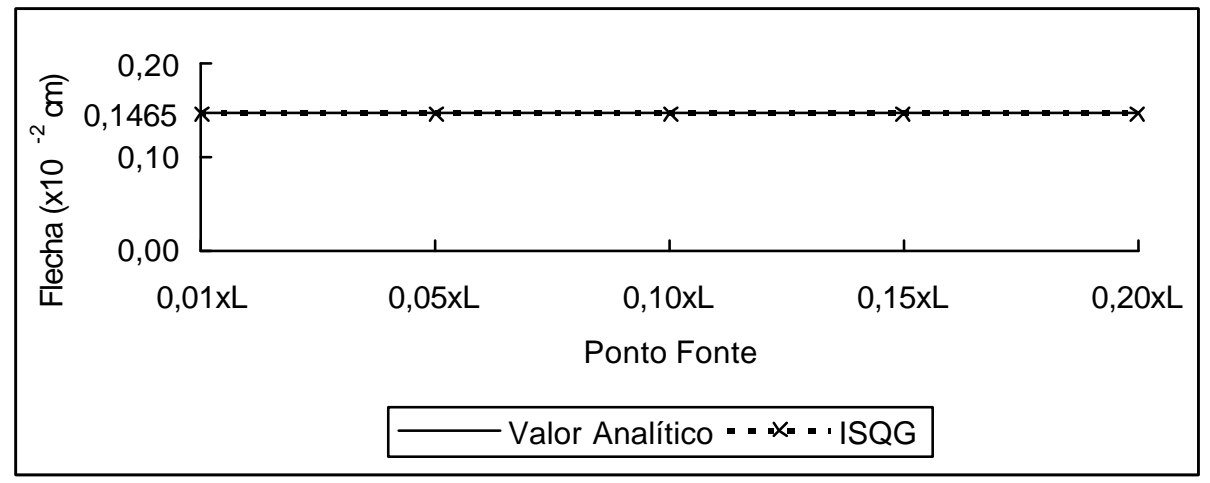

Gráfico 7.29 - Estabilidade da malha $2 \times 2-L=25 \mathrm{~cm}$ - elemento quadrático

Tabela 7.44 - Deslocamentos em placas retangulares com curvatura cilíndrica elemento quadrático

\begin{tabular}{ccccccc}
\hline \multicolumn{1}{c}{$\begin{array}{c}\mathrm{L} \\
(\mathrm{cm})\end{array}$} & $\begin{array}{c}\text { RELAÇÃO } \\
\mathrm{H}: \mathrm{L}\end{array}$ & $\begin{array}{c}\text { DESLOC. ANALÍTICO } \\
\mathrm{EM} \mathrm{X}_{3}\left(\times 10^{-2} \mathrm{~cm}\right)\end{array}$ & ISSA & ERRO (\%) & ISQG & ERRO (\%) \\
\hline & & & & & & \\
25,00 & $1: 2,5$ & 0,1465 & 0,1465 & 0,00 & 0,1465 & 0,00 \\
50,00 & $1: 5$ & 0,5859 & 0,5859 & 0,00 & 0,5858 & $-0,03$ \\
100,00 & $1: 10$ & 2,3437 & 2,3436 & $-0,01$ & 2,3436 & $-0,01$ \\
& & & & & & \\
\hline
\end{tabular}

Tabela 7.45 - Número de condicionamento - curvatura cilíndrica - elemento quadrático

\begin{tabular}{ccrr}
\hline $\mathrm{L}$ & $\begin{array}{c}\text { RELAÇÃO } \\
\mathrm{H}: \mathrm{cm})\end{array}$ & \multicolumn{2}{c}{$\begin{array}{c}\text { NÚMERO DE CONDICIONAMENTO } \\
\text { ISSA }\end{array}$} \\
\hline & & & \multicolumn{1}{c}{ ISQG } \\
25,00 & $1: 2,5$ & 71.904 & 12.284 .879 \\
50,00 & $1: 5$ & 57.039 & 851.824 \\
100,00 & $1: 10$ & 47.136 & 4.478 .909 \\
& & & \\
\hline
\end{tabular}

Como previsto, o elemento quadrático apresenta resultados excelentes para este tipo de problema, independente da relação H:L. O erro que aparece é devido ao cálculo numérico, e o condicionamento com o ponto fonte no contorno (ISSA) é bem melhor do que com o ponto fonte fora (ISQG). 


\subsubsection{Placa retangular com curvatura esférica}

Para a curvatura esférica foram mantidas as mesmas densidades de malhas e graus de liberdade da curvatura cilíndrica, pois o grau da resposta em deslocamento é o mesmo. A única diferença é na aplicação do carregamento e apoios, que podem ser vistos na figura 7.17.

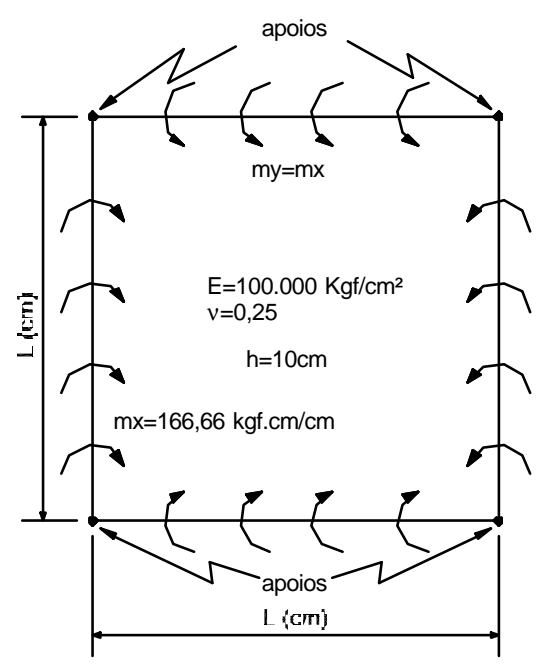

Figura 7.17 - Placa retangular com curvatura esférica - elemento linear

Também não irão se mostrar convergência e estabilidade, por estes já terem sido abordados em 7.8.1.

$\mathrm{Na}$ tabela 7.46 apresentam-se os valores de deslocamentos transversais para o ponto central da placa com curvatura esférica e na tabela 7.47 os números de condicionamento. $\mathrm{O}$ fator de ponto fora também foi mantido.

Tabela 7.46 - Deslocamentos em placas retangulares com curvatura esférica elemento linear

\begin{tabular}{|c|c|c|c|c|c|c|c|c|}
\hline \multirow{2}{*}{$\begin{array}{c}\mathrm{L} \\
(\mathrm{cm})\end{array}$} & \multicolumn{2}{|c|}{ RELAÇÃO DESLOC. ANALÍTICO } & \multicolumn{6}{|c|}{ FORMULAÇÃO } \\
\hline & $\mathrm{H}: \mathrm{L}$ & $\mathrm{EM} \mathrm{X}_{3}\left(\times 10^{-2} \mathrm{~cm}\right)$ & ISSA & ERRO (\%) & ISQG & ERRO (\%) & HMEC & ERRO (\%) \\
\hline 25,00 & $1: 2,5$ & 0,2344 & 0,2034 & $-13,22$ & 0,2230 & $-4,84$ & 0,2246 & $-4,16$ \\
\hline 50,00 & $1: 5$ & 0,9375 & 0,8027 & $-14,38$ & 0,8956 & $-4,47$ & 0,8971 & $-4,31$ \\
\hline 100,00 & $1: 10$ & 3,7500 & 2,5202 & $-32,79$ & 3,3518 & $-10,62$ & 3,3528 & $-10,59$ \\
\hline
\end{tabular}


Tabela 7.47 - Número de condicionamento - curvatura esférica - elemento linear

\begin{tabular}{ccccr}
\hline $\mathrm{L}$ & $\mathrm{RELAÇÃO}$ & \multicolumn{3}{l}{ NÚMERO DE CONDICIONAMENTO } \\
$(\mathrm{cm})$ & $\mathrm{H}: \mathrm{L}$ & ISSA & ISQG & HMEC \\
\hline & & & & \\
25,00 & $1: 2,5$ & 38.339 & 52.109 & 70.286 \\
50,00 & $1: 5$ & 29.699 & 34.065 & 54.648 \\
100,00 & $1: 10$ & 16.819 & 21.371 & 16.238 \\
& & & & \\
\hline
\end{tabular}

Como para a curvatura cilíndrica, os maiores erros apresentados foram os da formulação ISSA, o que já era previsto, o que mudou foi o condicionamento das formulações ISQG e HMEC, isto foi devido às condições de contorno impostas em deslocamentos, que conseguiu representar melhor o problema físico.

Para o elemento quadrático, também foram utilizadas as mesmas discretizações do problema anterior para este tipo de elemento. E os resultados de deslocamentos e números de condicionamento encontram-se nas tabelas $7.48 \mathrm{e}$ 7.49 , respectivamente.

Tabela 7.48 - Deslocamentos em placas retangulares com curvatura esférica elemento quadrático

\begin{tabular}{ccccccc}
\hline \multicolumn{1}{c}{$\begin{array}{c}\mathrm{R} \\
(\mathrm{cm})\end{array}$} & $\begin{array}{c}\text { RELAÇÃO } \\
\mathrm{H}: \mathrm{L}\end{array}$ & $\begin{array}{c}\text { DESLOC. ANALÍTICO } \\
\text { EM } \mathrm{X}_{3}\left(\times 10^{-2} \mathrm{~cm}\right)\end{array}$ & ISSA & ERRO (\%) & ISQG & ERRO (\%) \\
\hline & & & & & & \\
25,00 & $1: 2,5$ & 0,2344 & 0,2344 & 0,00 & 0,2344 & 0,00 \\
50,00 & $1: 5$ & 0,9375 & 0,9374 & $-0,01$ & 0,9374 & $-0,01$ \\
100,00 & $1: 10$ & 3,7500 & 3,7499 & 0,00 & 3,7455 & $-0,12$ \\
& & & & & & \\
\hline
\end{tabular}

Tabela 7.49 - Número de condicionamento - curvatura esférica - elemento quadrático

\begin{tabular}{ccrr}
\hline $\begin{array}{c}\mathrm{L} \\
(\mathrm{cm})\end{array}$ & $\begin{array}{c}\text { RELAÇÃO } \\
\mathrm{H}: \mathrm{L}\end{array}$ & \multicolumn{2}{c}{$\begin{array}{c}\text { NÚMERO DE CONDICIONAMENTO } \\
\text { ISSA }\end{array}$} \\
\hline & & & \multicolumn{1}{c}{ ISQG } \\
25,00 & $1: 2,5$ & 48.903 & 21.258 .877 \\
50,00 & $1: 5$ & 33.165 & 449.102 \\
100,00 & $1: 10$ & 15.897 & 757.799 \\
& & & \\
\hline
\end{tabular}

Tal qual a curvatura cilíndrica, o elemento quadrático também não apresentou erro, a não ser numérico, e os números de condicionamento da formulação ISSA são bem melhores do que a formulação ISQG. 


\subsubsection{Placa retangular com momentos uniformes em duas bordas}

Este exemplo é análogo aos outros dois anteriores, portanto as discretizações e o número de graus de liberdade são mantidos. Na figura 7.18 apresenta-se o carregamento aplicado e as condições de contorno em deslocamentos.

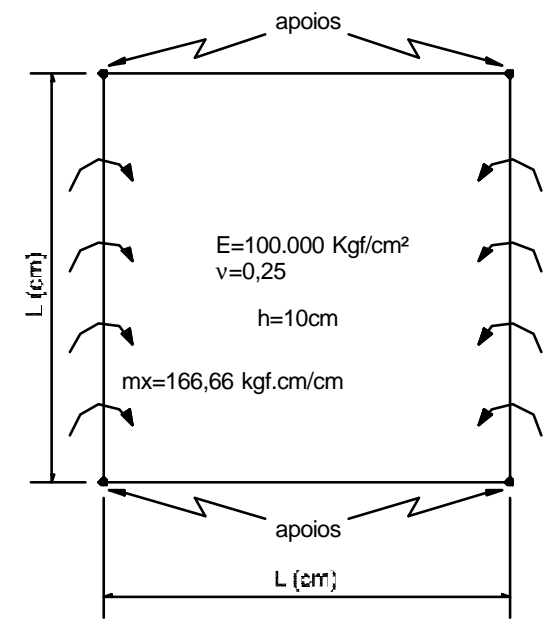

Figura 7.18 - Placa retangular com momentos uniformes em duas bordas - elemento linear

As discretizações e números de graus de liberdade foram mantidos para este exemplo. Na tabela 7.50 apresentam-se os resultados de deslocamentos transversais no ponto central da placa e na tabela 7.51 os números de condicionamento. Neste caso também se manteve o fator de ponto fora.

Tabela 7.50 - Deslocamentos em placas retangulares com momentos uniformes em duas bordas - elemento linear

\begin{tabular}{ccccccccc}
\hline \multicolumn{1}{c}{$\mathrm{L}$} & \multicolumn{2}{c}{ RELAÇÃO DESLOC. ANALÍTICO } & \multicolumn{5}{c}{ FORMULAÇÃO } \\
$(\mathrm{cm})$ & $\mathrm{H}: \mathrm{L}$ & $\mathrm{EM} \mathrm{X}_{3}\left(\times 10^{-2} \mathrm{~cm}\right)$ & ISSA & ERRO (\%) & ISQG & ERRO (\%) & HMEC & ERRO (\%) \\
\hline & & & & & & & & \\
25,00 & $1: 2,5$ & 0,1172 & 0,1017 & $-13,22$ & 0,1133 & $-3,28$ & 0,1125 & $-3,99$ \\
50,00 & $1: 5$ & 0,4687 & 0,4013 & $-14,39$ & 0,4496 & $-4,08$ & 0,4487 & $-4,28$ \\
100,00 & $1: 10$ & 1,8750 & 1,2601 & $-32,79$ & 1,6776 & $-10,53$ & 1,6763 & $-10,60$ \\
& & & & & & & & \\
\hline
\end{tabular}


Tabela 7.51 - Número de condicionamento - placas retangulares com momentos uniformes em duas bordas - elemento linear

\begin{tabular}{ccccr}
\hline $\mathrm{L}$ & $\mathrm{R} E L A C ̧ A ̃ O$ & \multicolumn{3}{c}{ NÚMERO DE CONDICIONAMENTO } \\
$(\mathrm{cm})$ & $\mathrm{H}: \mathrm{L}$ & ISSA & ISQG & HMEC \\
\hline & & & & \\
25,00 & $1: 2,5$ & 38.339 & 52.109 & 70.286 \\
50,00 & $1: 5$ & 29.699 & 34.065 & 54.648 \\
100,00 & $1: 10$ & 16.819 & 21.371 & 16.238 \\
& & & & \\
\hline
\end{tabular}

Os erros foram análogos aos exemplos anteriores, justamente por este exemplo apresentar o mesmo tipo de comportamento.

Para o elemento quadrático também se mantiveram todos os parâmetros dos dois exemplos anteriores resolvidos com este elemento. $\mathrm{Na}$ tabela 7.52 apresentam-se os deslocamentos transversais e na tabela 7.53 os números de condicionamento.

Tabela 7.52 - Deslocamentos em placas retangulares com momentos uniformes em duas bordas - elemento quadrático

\begin{tabular}{ccccccc}
\hline $\begin{array}{c}\mathrm{L} \\
(\mathrm{cm})\end{array}$ & $\begin{array}{c}\text { RELAÇÃO } \\
\mathrm{H}: \mathrm{L}\end{array}$ & $\begin{array}{c}\text { DESLOC. ANALÍTICO } \\
\text { EM } x_{3}\left(\times 10^{-2} \mathrm{~cm}\right)\end{array}$ & ISSA & ERRO (\%) & ISQG & ERRO (\%) \\
\hline & & & & & & \\
25,00 & $1: 2,5$ & 0,1172 & 0,1172 & 0,00 & 0,1172 & 0,00 \\
50,00 & $1: 5$ & 0,4687 & 0,4687 & $-0,01$ & 0,4686 & $-0,02$ \\
100,00 & $1: 10$ & 1,8750 & 1,8749 & $-0,01$ & 1,8732 & $-0,10$ \\
& & & & & & \\
\hline
\end{tabular}

Tabela 7.53 - Número de condicionamento - placas retangulares com momentos uniformes em duas bordas - elemento quadrático

\begin{tabular}{rlrr}
\hline $\begin{array}{c}\mathrm{L} \\
(\mathrm{cm})\end{array}$ & $\begin{array}{c}\text { RELAÇÃO } \\
\mathrm{H}: \mathrm{L}\end{array}$ & $\begin{array}{c}\text { NÚMERO DE CONDICIONAMENTO } \\
\text { ISSA }\end{array}$ & \multicolumn{1}{c}{ ISQG } \\
\hline & & & \\
25,00 & $1: 2,5$ & 48.903 & 21.258 .877 \\
50,00 & $1: 5$ & 33.165 & 449.102 \\
100,00 & $1: 10$ & 15.897 & 757.799 \\
& & & \\
\hline
\end{tabular}

Como para os outros exemplos, pois este problema possui o mesmo comportamento, o elemento quadrático não apresenta erro, a não ser numérico. 


\subsection{Exemplo 8 - Placa quadrada simplesmente apoiada com carga uniformemente distribuída}

Neste exemplo examinam-se com placas quadradas com diversas relações entre espessura e lado, simplesmente apoiadas nas quatro bordas (apoios no plano médio das placas) e com carregamento uniformemente distribuído (flexão simples). As placas possuem propriedades geométricas e físicas de acordo com a figura 7.19. Este exemplo foi resolvido apenas com o elemento quadrático, pois se fosse utilizado o elemento linear, haveria a necessidade de uma enorme discretização para que os resultados fossem confiáveis e aceitáveis. Os resultados obtidos pelo MEC-3D foram comparados com os resultados obtidos por Ribeiro (1976) apud Ribeiro (1992), por este levar em consideração o efeito do esforço cortante no cálculo de deslocamentos.

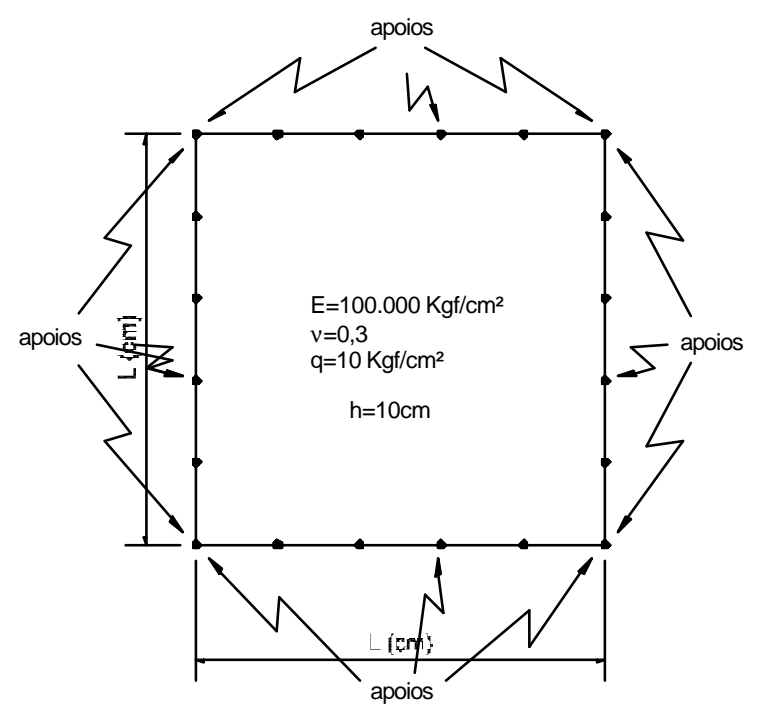

Figura 7.19 - Placa quadrada simplesmente apoiada

As placas analisadas possuem os seguintes vãos e discretizações:

$\mathrm{L}=40 \mathrm{~cm}$ - malha $4 \times 4-1098 \mathrm{GL}$ e bordas discretizadas de acordo com a figura 7.20;

$\mathrm{L}=50 \mathrm{~cm}$ - malha $8 \times 8$ - $2346 \mathrm{GL}$ e bordas com o mesmo número de elementos; $\mathrm{L}=100 \mathrm{~cm}$ - malha $10 \times 10-3258 \mathrm{GL}$ e bordas com o mesmo número de elementos. 


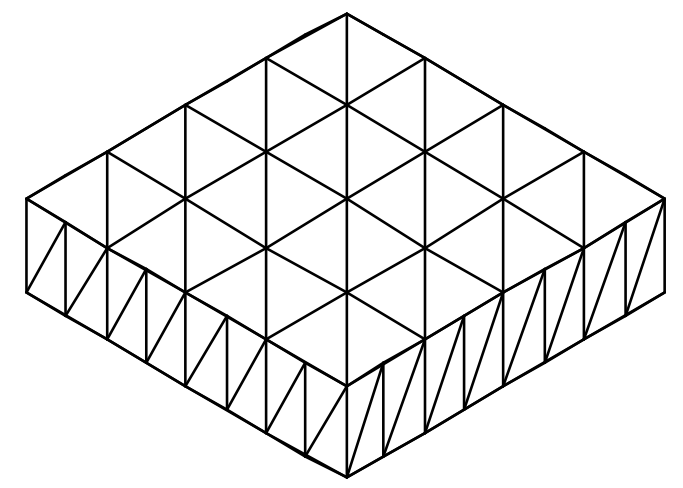

Figura 7.20 - Discretização da placa quadrada $L=40 \mathrm{~cm}$ - malha $4 \times 4$ - elemento quadrático

No gráfico 7.30 é apresentada a convergência do elemento quadrático para a placa com vão $L=40 \mathrm{~cm}$, utilizando-se o fator de ponto fora com valor 0,1 .

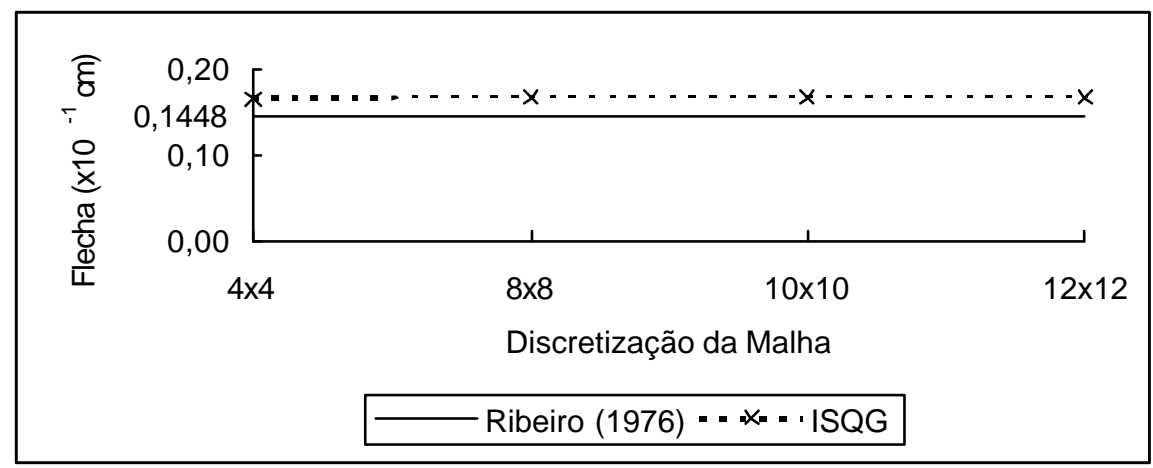

Gráfico 7.30 - Convergência da placa retangular simplesmente apoiada sob flexão simples $-\mathrm{L}=40 \mathrm{~cm}$ - elemento quadrático

Como não houve grande variação nos resultados, escolheu-se a malha $4 \times 4$ para se realizar toda a análise. No gráfico 7.31 mostra-se a estabilidade da solução para a malha escolhida.

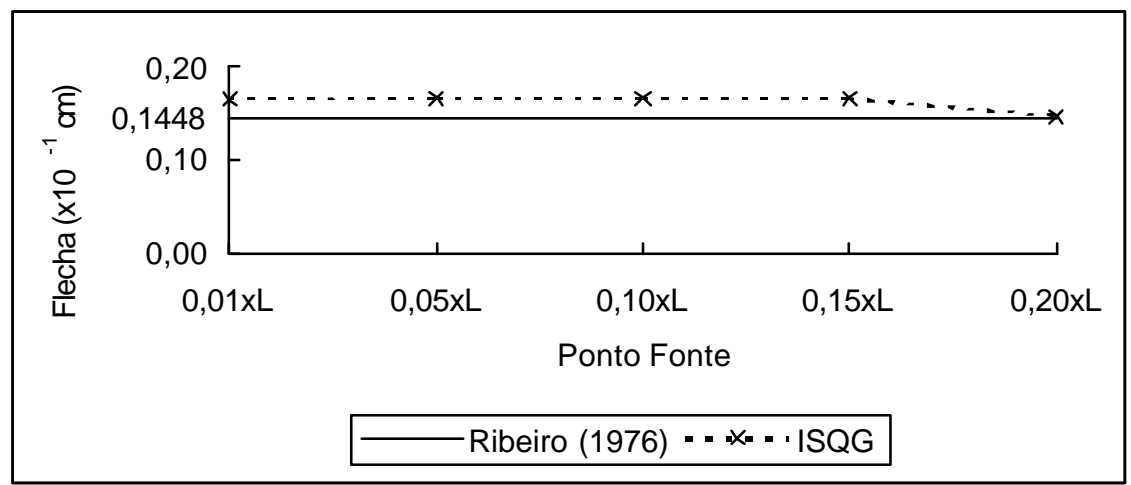

Gráfico 7.31 - Estabilidade da malha $4 \times 4$ - L=40cm - elemento quadrático 
Pelo gráfico 7.31 que mostra a estabilidade verifica-se que não há variabilidade, a não ser para o ponto fonte localizado a $0,2 \mathrm{~L}$ do elemento, o que não mostra que este valor é o correto. A seguir, na tabela 7.54 , são comparados os fatores $w \cdot\left(\frac{100 \cdot D}{q \cdot L^{4}}\right)$ para as placas com diversos vãos, e na tabela 7.55 mostram-se os números de condicionamento. Utiliza-se o valor de 0,1 para o fator da técnica do ponto fora, pois como 0,2 é o único ponto destoante, os resultados com este fator não poderiam de maneira alguma ser confiáveis, apesar de, provavelmente, apresentar, máximos valores dos termos cruzados da matriz $\mathrm{H}$, importantes para esta análise.

Tabela 7.54 - Comparação dos fatores $w\left(100 \mathrm{D} / \mathbf{q l}^{4}\right)$ - elemento quadrático

\begin{tabular}{cccccccc}
\hline $\begin{array}{c}\mathrm{L} \\
(\mathrm{cm})\end{array}$ & $\begin{array}{c}\text { RELAÇÃO } \\
\mathrm{H}: \mathrm{L}\end{array}$ & $\begin{array}{c}\text { Ribeiro } \\
(1976)\end{array}$ & $\begin{array}{c}\text { Ribeiro - MEC } \\
(1992)\end{array}$ & ISSA & \multicolumn{2}{c}{ FORMULAÇÃO } \\
ERRO (\%) & ISQG & ERRO (\%) \\
\hline & & & & & & & \\
40,00 & $1: 4$ & 0,518 & 0,5182 & 0,6042 & 16,60 & 0,5901 & 13,87 \\
50,00 & $1: 5$ & 0,478 & 0,4779 & 0,5448 & 13,99 & 0,5439 & 13,80 \\
100,00 & $1: 10$ & 0,424 & 0,4241 & 0,4507 & 6,26 & 0,4628 & 9,11 \\
\hline
\end{tabular}

Tabela 7.55 - Número de condicionamento - exemplo 8 - elemento quadrático

\begin{tabular}{|c|c|c|c|}
\hline \multirow{2}{*}{$\begin{array}{c}\mathrm{L} \\
(\mathrm{cm})\end{array}$} & \multirow{2}{*}{$\begin{array}{c}\text { RELAÇÃO } \\
H: L\end{array}$} & \multicolumn{2}{|c|}{ NÚMERO DE CONDICIONAMENTO } \\
\hline & & ISSA & ISQG \\
\hline 40,00 & $1: 4$ & 927.192 .065 & 33.640 .040 \\
\hline 50,00 & $1: 5$ & 999.999.999.999 & 2.702.036.693 \\
\hline 100,00 & $1: 10$ & 999.999.999.999 & 77.169 .923 \\
\hline
\end{tabular}

Como previsto, os resultados pelo MEC-3D estão muito aquém do que se esperava, pois se utilizando o elemento quadrático seria necessária uma discretização absurda para que os resultados se tornassem aceitáveis e estáveis, pois a resposta do problema é do $4^{\circ}$ grau. 


\section{CAPÍTULO 8}

\section{CONCLUSÕES}

Neste trabalho abordou-se o problema elástico tridimensional pelo método dos elementos de contorno com novas formulações além da clássica. Foram desenvolvidas integrais analíticas para o elemento constante, e semi-analíticas para os elementos constante, linear e quadrático. As integrais analíticas agilizam o processo de integração, incrementando a velocidade de processamento.

Observou-se, tanto para a formulação clássica quanto para a formulação hiper-singular, que, quando se utilizam malhas pouco densas (pobres) os resultados obtidos pelo MEC apresentam grande dependência em relação à posição do ponto fonte.

Este problema está diretamente relacionado a dois fatores. O primeiro, já comentado, diz respeito à densidade de malha e grau de aproximação das variáveis empregados. O segundo está relacionado àqualidade das integrais efetuadas.

A utilização do processo de integração via quadratura de Hammer e divisão em subelementos triangulares de mesmo tamanho se mostrou ineficiente, tanto em tempo de processamento quanto na precisão das integrais quase singulares executadas, não resolvendo o problema da dependência dos resultados com relação àposição do ponto fonte.

Para contornar tal situação desenvolveu-se processo de integração de elementos quase singulares baseado em coordenadas polares adimensionais e subdivisão progressiva do raio conforme Mom-ma et al. (1996).

Como conseqüência obtiveram-se resultados estáveis para qualquer posição do ponto fonte, desde que sua distância ao elemento de contorno quase singular 
seja menor que metade do máximo comprimento dos lados deste elemento, condição que garante a boa representação integral do problema.

A estabilidade conseguida pela aplicação das técnicas de integração singular e quase singular, desenvolvidas neste trabalho, obviamente ocorre quando o fator densidade de discretização é atendido.

No início do trabalho acreditava-se que o uso de elementos de contorno com aproximação constante e com integrais singulares bem feitas resultaria em um processo numérico estável e ótimo. Os exemplos revelaram o contrário, ou seja, o uso de aproximação constante não consegue atingir estabilidade na solução, apesar de se conseguir convergência nos resultados para pontos de colocação localizada em distâncias específica, como atesta o exemplo de sólido sob força transversal. O uso de integrais singulares neste caso foi uma catástrofe devido à ausência de termos cruzados na região da diagonal principal da matriz $\mathrm{H}$.

Em seguida imaginou-se que o uso de soluções hiper-singulares ou de formulações baseadas em forças concentradas melhoraria o condicionamento do sistema de equações e conseqüentemente os resultados. Concluiu-se que a qualidade dos resultados nos sistemas lineares resolvidos não depende significativamente do número de condicionamento.

Neste momento notou-se que os resultados e sua estabilidade dependiam exclusivamente da densidade de malha e da qualidade de integração.

Assim, implementaram-se os elementos linear e quadrático. $O$ uso destes elementos possibilitou a análise de convergência e estabilidade (com relação à posição do ponto fonte) dos exemplos processados, concluindo-se que o melhor uso do método se dá com o uso de integrais singulares ou quase singulares, pois estes apresentam um melhor condicionamento, desde que a discretização seja bem feita.

Conclui-se que o elemento quadrático apresenta taxa de convergência e nível de estabilidade superior ao elemento linear, principalmente nos exemplos de placas submetidas àflexão.

Descarta-se a utilização do elemento constante devido ao não preenchimento da condição de estabilidade.

Recomenda-se, além do teste de convergência para uma progressão de malhas, a verificação da estabilidade dos resultados com relação à posição do ponto fonte para se aceitar um resultado, supostamente correto, como verdadeiro. 
Caso se escolha uma malha bastante densa, de acordo com a aproximação escolhida, basta verificar a invariabilidade dos resultados com relação àlocalização do ponto fonte.

Como recomendação final deve-se ter cuidado ao se observar resultados científicos baseados no MEC, quando a densidade de malha for pobre.

Como comentário geral, acredita-se que na literatura superestima-se a capacidade de aproximação do MEC quando se observam as densidades de malha aplicadas.

Com tudo o que foi apresentado e concluído nesta pesquisa, pode-se no futuro implementar um procedimento automático para a escolha da malha que conduza a resultados confiáveis. E com isso produzir um software, cuja utilização por usuários com pouco conhecimento sobre o assunto seja simples e eficiente. Pesquisas voltadas ao estudo das propriedades espectrais da matriz $\mathrm{G}$ devem ser executados no futuro, tentando avaliar as perturbações introduzidas nos resultados ao se prescrever deslocamentos. 


\section{ANEXO A}

\section{Valor principal de Cauchy para o elemento constante}

Para obter o valor principal de Cauchy da integral (6.59), é necessário substituir o limite inferior da integral por um raio $\varepsilon$, como pode ser visto na figura A.1, e fazer o limite deste tendendo a zero:

$$
\underset{\sim}{h}=\int_{\theta_{i}}^{\theta_{f}} \frac{(1-2 \cdot v)}{8 \cdot \pi \cdot(1-v)} \cdot\left(r,{ }_{i} \cdot \eta_{j}-r,{ }_{j} \cdot \eta_{i}\right) \cdot\left[\ln R-\lim _{\varepsilon \rightarrow 0}(\ln \varepsilon)\right] \cdot d \theta
$$

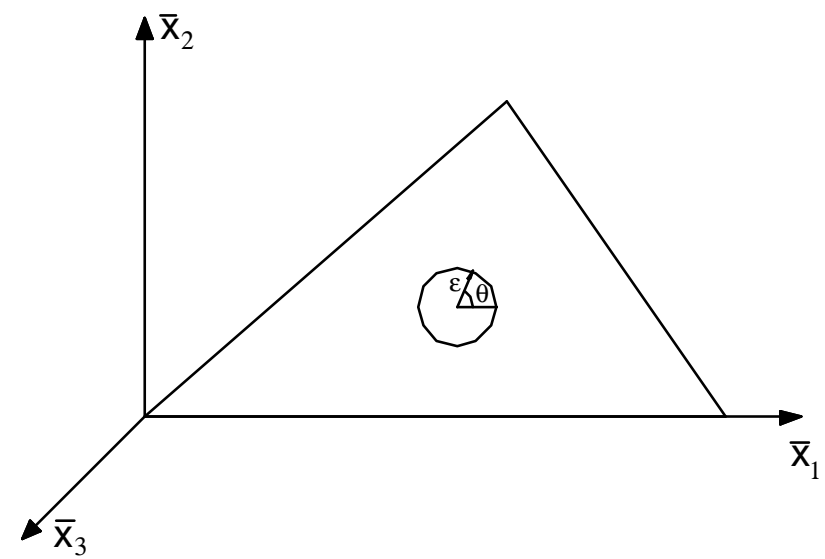

Figura A.1 - Valor principal de Cauchy para o elemento constante

Substituindo-se a parte constante por $\mathrm{K}$ e desmembrando-se a integral em duas partes: 


$$
\underset{\sim}{h}=K \int_{\theta_{i}}^{\theta_{f}}\left(r,{ }_{i} \cdot \eta_{j}-r,{ }_{j} \cdot \eta_{i}\right) \cdot \ln R \cdot d \theta-K \cdot \int_{\theta_{i}}^{\theta_{f}}\left(r,{ }_{i} \cdot \eta_{j}-r,{ }_{j} \cdot \eta_{i}\right) \cdot \lim _{\varepsilon \rightarrow 0}(\ln \varepsilon) \cdot d \theta \quad \text { ( A.2 ) }
$$

Agora basta demonstrar que a segunda parte da integral (A.2) tende a zero quando $\varepsilon$ tender a zero. De acordo com a figura A.1, a segunda parte da integral (A.2) assume o seguinte aspecto:

$-K \cdot \int_{0}^{2 \pi}\left(r_{, i} \cdot \eta_{j}-r,{ }_{j} \cdot \eta_{i}\right) \cdot d \theta \cdot \lim _{\varepsilon \rightarrow 0}(\ln \varepsilon)$

Para demonstrar que essa integral vale zero, basta demonstrar que:

$$
\int_{0}^{2 \pi}\left(r,{ }_{i} \cdot \eta_{j}-r,{ }_{j} \cdot \eta_{i}\right) \cdot d \theta=0
$$

Para isso substituem-se as diferenciais do raio em relação às coordenadas globais (6.55). Caso $i=j$ tem-se diretamente que:

$$
\int_{0}^{2 \pi}\left(r,,_{i} \cdot \eta_{i}-r,{ }_{i} \cdot \eta_{i}\right) \cdot d \theta=0
$$

Caso $i \neq j$ tem-se que:

$$
\begin{aligned}
& \int_{0}^{2 \pi}\left[\left(\cos \theta \cdot \frac{\partial \bar{X}_{1}}{\partial X_{i}}+\operatorname{sen} \theta \cdot \frac{\partial \bar{X}_{2}}{\partial X_{i}}\right) \cdot \eta_{j}-\left(\cos \theta \cdot \frac{\partial \bar{X}_{1}}{\partial X_{j}}+\operatorname{sen} \theta \cdot \frac{\partial \bar{X}_{2}}{\partial X_{j}}\right) \cdot \eta_{i}\right] \cdot d \theta \\
& =\left[\left(\operatorname{sen} \theta \cdot \frac{\partial \bar{X}_{1}}{\partial X_{i}}-\cos \theta \cdot \frac{\partial \bar{X}_{2}}{\partial X_{i}}\right) \cdot \eta_{j}-\left(\operatorname{sen} \theta \cdot \frac{\partial \bar{X}_{1}}{\partial X_{j}}-\cos \theta \cdot \frac{\partial \bar{X}_{2}}{\partial X_{j}}\right) \cdot \eta_{i}\right]_{0}^{2 \pi} \\
& =0
\end{aligned}
$$

Portanto:

$$
-K \cdot \int_{0}^{2 \pi}\left(r,{ }_{i} \cdot \eta_{j}-r,{ }_{j} \cdot \eta_{i}\right) \cdot d \theta \cdot \lim _{\varepsilon \rightarrow 0}(\ln \varepsilon)=0 \quad \text { (C.Q.D.) }
$$




\section{Valor principal de Cauchy para o elemento linear}

Procedendo-se da mesma forma que para o elemento constante, integrandose (6.68), tem-se a seguinte expressão:

$$
\begin{aligned}
h_{i j}= & \int_{\theta_{i}}^{\theta_{f}} \frac{(1-2 \cdot v)}{8 \cdot \pi \cdot(1-v)} \cdot\left(r,{ }_{i} \cdot \eta_{j}-r,{ }_{j} \cdot \eta_{i}\right) . \\
& {\left[\xi_{k}^{S} \cdot\left(\ln R-\lim _{\varepsilon \rightarrow 0}(\ln \varepsilon)\right)+\frac{R}{2 \cdot A} \cdot\left(b^{k} \cdot \cos \theta+a^{k} \cdot \operatorname{sen} \theta\right)\right] \cdot d \theta }
\end{aligned}
$$

Dessa forma é necessário demonstrar que a parte que possui o limite de $\varepsilon$ tende a zero quando $\varepsilon$ tender a zero. Assim tem-se a seguinte integral:

$$
\int_{\theta_{i}}^{\theta_{f}}\left(r, \cdot \eta_{j}-r,{ }_{j} \cdot \eta_{i}\right) \cdot\left[-\xi_{k}^{S} \cdot \lim _{\varepsilon \rightarrow 0}(\ln \varepsilon)\right] \cdot d \theta
$$

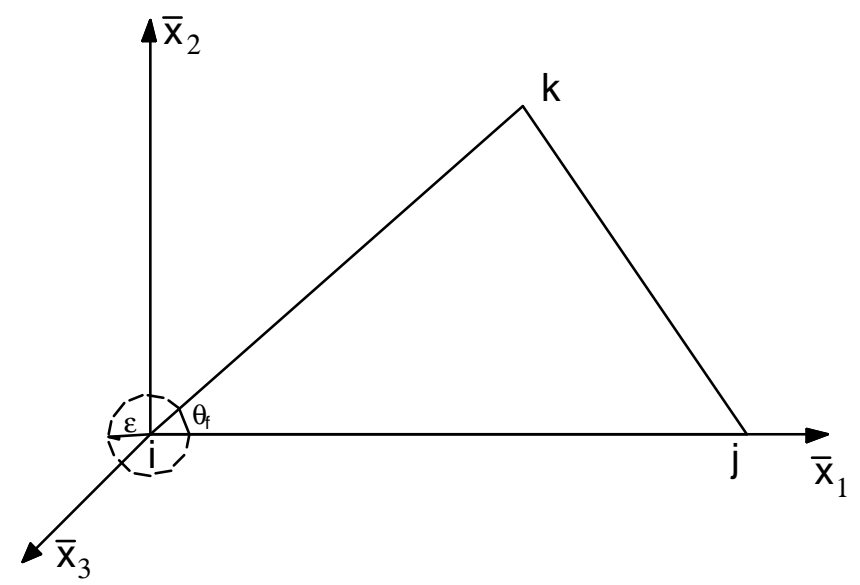

Figura A.2 - Valor principal de Cauchy para o elemento linear

De acordo com a figura A.2, tem-se que:

$$
-\xi_{k}^{S} \cdot \int_{0}^{\theta_{f}}\left(r,{ }_{i} \cdot \eta_{j}-r,{ }_{j} \cdot \eta_{i}\right) \cdot d \theta \cdot\left[\lim _{\varepsilon \rightarrow 0}(\ln \varepsilon)\right]
$$

Tomando-se a soma de todos os elementos que possuem como ponto fonte 0 ponto i tem-se que $\theta_{\mathrm{f}}=2 \pi$. Portanto basta demonstrar que: 


$$
\int_{0}^{2 \pi}\left(r,{ }_{i} \cdot \eta_{i}-r,{ }_{i} \cdot \eta_{i}\right) \cdot d \theta=0
$$

Como se demonstrou anteriormente, essa integral vale zero, portanto:

$$
\int_{\theta_{i}}^{\theta_{f}}\left(r,{ }_{i} \cdot \eta_{j}-r,{ }_{j} \cdot \eta_{i}\right) \cdot\left[-\xi_{k}^{S} \cdot \lim _{\varepsilon \rightarrow 0}(\ln \varepsilon)\right] \cdot d \theta=0 \quad \text { (C.Q.D.) }
$$




\section{Integrais com variável em $\theta$}

Neste anexo são mostradas as primitivas das integrais com variável em $\theta$ a solução das integrais singulares (6.51) e (6.52).

Integral (1):

$\int \frac{1}{b^{k} \cdot \cos \theta+a^{k} \cdot \operatorname{sen} \theta} \cdot d \theta=\frac{1}{\sqrt{\left(a^{k}\right)^{2}+\left(b^{k}\right)^{2}}} \cdot \ln \mid \frac{b \cdot \operatorname{tg}\left(\frac{\theta}{2}\right)-a^{k}+\sqrt{\left(a^{k}\right)^{2}+\left(b^{k}\right)^{2}}}{b \cdot \operatorname{tg}\left(\frac{\theta}{2}\right)-a^{k}-\sqrt{\left(a^{k}\right)^{2}+\left(b^{k}\right)^{2}}}+c$

( B.1)

Onde:

$$
\sqrt{\left(a^{k}\right)^{2}+\left(b^{k}\right)^{2}}=\mathrm{L}_{\mathrm{ml}}
$$

Esta integral possui uma descontinuidade para $b^{k}=0$ e para $\theta=\pi / 2$. para 0 primeiro caso é fácil verificar que o lado direito da igualdade fica dessa forma:

$$
\int \frac{1}{b^{k} \cdot \cos \theta+a^{k} \cdot \operatorname{sen} \theta} \cdot d \theta=\int \frac{1}{a^{k} \cdot \operatorname{sen} \theta} \cdot d \theta=-\frac{1}{a^{k}} \cdot \ln \left|\frac{1}{\operatorname{sen} \theta}+\frac{1}{\operatorname{tg} \theta}\right|
$$

Já o segundo caso é preciso verificar o limite quando $\theta \rightarrow \pi / 2$ : 
$\lim _{\theta \rightarrow \frac{\pi}{2}}\left(\ln \left|\frac{b \cdot \operatorname{tg}\left(\frac{\theta}{2}\right)-a^{k}+\sqrt{\left(a^{k}\right)^{2}+\left(b^{k}\right)^{2}}}{b \cdot \operatorname{tg}\left(\frac{\theta}{2}\right)-a^{k}-\sqrt{\left(a^{k}\right)^{2}+\left(b^{k}\right)^{2}}}\right|\right)=\lim _{\theta \rightarrow \frac{\pi}{2}}\left(\ln \mid \frac{b \cdot \operatorname{tg}\left(\frac{\theta}{2}\right)}{b \cdot \operatorname{tg}\left(\frac{\theta}{2}\right)}\right)=1$

Para obter este limite é só aplicar a regra de L'Hospital.

Integral (2):

$$
\begin{aligned}
\int \frac{\cos ^{2} \theta}{b^{k} \cdot \cos \theta+a^{k} \cdot \operatorname{sen} \theta} \cdot d \theta= & \frac{2 \cdot a^{k} \cdot \cos ^{2}\left(\frac{\theta}{2}\right)}{\left[\left(a^{k}\right)^{2}+\left(b^{k}\right)^{2}\right]}+\frac{b^{k} \cdot \operatorname{sen} \theta}{\left[\left(a^{k}\right)^{2}+\left(b^{k}\right)^{2}\right]}+ \\
& \frac{\left(a^{k}\right)^{2}}{\left[\left(a^{k}\right)^{2}+\left(b^{k}\right)^{2}\right]^{\frac{3}{2}}} \cdot \ln \mid \frac{b \cdot \operatorname{tg}\left(\frac{\theta}{2}\right)-a^{k}+\sqrt{\left(a^{k}\right)^{2}+\left(b^{k}\right)^{2}}}{b \cdot \operatorname{tg}\left(\frac{\theta}{2}\right)-a^{k}-\sqrt{\left(a^{k}\right)^{2}+\left(b^{k}\right)^{2}} \mid+c}
\end{aligned}
$$

( B.4 )

Esta integral também apresenta descontinuidade para $b^{k}=0$ e $\theta=\pi / 2$, o limite da parte logarítmica foi mostrado anteriormente:

$$
\int \frac{\cos ^{2} \theta}{b^{k} \cdot \cos \theta+a^{k} \cdot \operatorname{sen} \theta} \cdot d \theta=\int \frac{\cos ^{2} \theta}{a^{k} \cdot \operatorname{sen} \theta} \cdot d \theta=\frac{1}{a^{k}}\left[-\ln \left(\frac{1}{\operatorname{sen} \theta}+\frac{1}{\operatorname{tg} \theta}\right)+\cos \theta\right]
$$

Integral (3):

$$
\begin{aligned}
\int \frac{\operatorname{sen}^{2} \theta}{b^{k} \cdot \cos \theta+a^{k} \cdot \operatorname{sen} \theta} \cdot d \theta & =-\frac{2 \cdot a^{k} \cdot \cos ^{2}\left(\frac{\theta}{2}\right)}{\left[\left(a^{k}\right)^{2}+\left(b^{k}\right)^{2}\right]}-\frac{b^{k} \cdot \operatorname{sen} \theta}{\left[\left(a^{k}\right)^{2}+\left(b^{k}\right)^{2}\right]}+ \\
& \frac{\left(b^{k}\right)^{2}}{\left[\left(a^{k}\right)^{2}+\left(b^{k}\right)^{2}\right]^{\frac{3}{2}}} \cdot \ln \left|\frac{b \cdot \operatorname{tg}\left(\frac{\theta}{2}\right)-a^{k}+\sqrt{\left(a^{k}\right)^{2}+\left(b^{k}\right)^{2}}}{b \cdot \operatorname{tg}\left(\frac{\theta}{2}\right)-a^{k}-\sqrt{\left(a^{k}\right)^{2}+\left(b^{k}\right)^{2}}}\right|+c
\end{aligned}
$$

Esta integral só possui descontinuidade para $\theta=\pi / 2$, e o limite da parte logarítmica já foi mostrada anteriormente. 
Integral (4):

$$
\begin{aligned}
\int \frac{\operatorname{sen} \theta \cdot \cos \theta}{b^{k} \cdot \cos \theta+a^{k} \cdot \operatorname{sen} \theta} \cdot d \theta & =-\frac{2 \cdot b^{k} \cdot \cos ^{2}\left(\frac{\theta}{2}\right)}{\left[\left(a^{k}\right)^{2}+\left(b^{k}\right)^{2}\right]}+\frac{a^{k} \cdot \operatorname{sen} \theta}{\left[\left(a^{k}\right)^{2}+\left(b^{k}\right)^{2}\right]}- \\
& \frac{b^{k} \cdot a^{k}}{\left[\left(a^{k}\right)^{2}+\left(b^{k}\right)^{2}\right]^{\frac{3}{2}}} \cdot \ln \left|\frac{b \cdot \operatorname{tg}\left(\frac{\theta}{2}\right)-a^{k}+\sqrt{\left(a^{k}\right)^{2}+\left(b^{k}\right)^{2}}}{b \cdot \operatorname{tg}\left(\frac{\theta}{2}\right)-a^{k}-\sqrt{\left(a^{k}\right)^{2}+\left(b^{k}\right)^{2}}}\right|+c
\end{aligned}
$$

Esta integral, como as outras, possui descontinuidade para $\theta=\pi / 2$.

Todas essas integrais foram obtidas através da técnica de integração que substitui $z=\operatorname{tg}(\theta / 2)$ e em seguida a técnica de frações parciais. 


\section{ANEXOC}

\section{Formulação do MEC para forças concentradas}

No intuito de melhorar o condicionamento do sistema linear, chegou-se a esta formulação com forças concentradas, consiste em aplicar forças pontuais e não, de forças distribuídas, como é o método clássico dos elementos de contorno. Para isso se faz uso da propriedade (3.21) do Delta de Dirac. Desenvolvendo a parte da Identidade Somigliana que contém forças reais, tem-se:

$$
\begin{aligned}
& \int_{\Gamma(Q)} u^{*}(s, Q) \cdot p(Q) \cdot d \Gamma(Q)=\int_{\Gamma(Q)} u^{*}(s, Q) \cdot P(S) \cdot \delta(S, Q) \cdot d \Gamma(Q) \\
& =\int_{\Gamma(Q)} u^{*}(s, Q) \cdot \delta(S, Q) \cdot d \Gamma(Q) \cdot P(S)=u^{*}(s, S) \cdot P(S)
\end{aligned}
$$

Com o ponto $\mathrm{S}$ fora do contorno e o ponto $\mathrm{S}$ no contorno, como mostra a figura C.1.

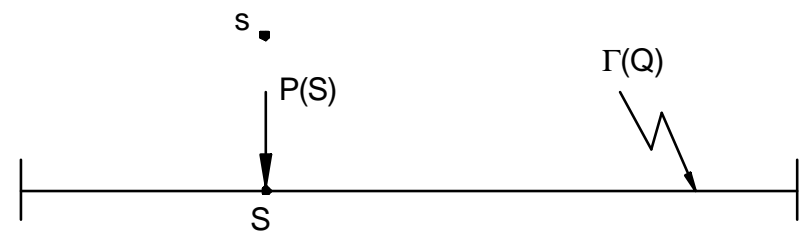

Figura A.3 - Formulação com forças concentradas

Sendo assim, a Identidade Somigliana assume o seguinte aspecto:

$$
\int_{\Gamma} u_{j}(Q) \cdot p_{i j}^{*}(s, Q) \cdot d \Gamma(Q)=P_{j}(S) \cdot u_{i j}^{*}(s, S)+\int_{\Omega} b_{j}(q) \cdot u_{i j}^{*}(s, q) \cdot d \Omega(q)
$$




\section{REFERÊNCIAS BIBLIOGRÁFICAS}

BARBIRATO, J. C. C. (1999). Método dos elementos de contorno com a reciprocidade dual para a análise transiente tridimensional da mecânica do fraturamento. São Carlos, Tese (Doutorado) - Escola de engenharia de São Carlos, Universidade de São Paulo.

BREBBIA, C. A.; (1978). The boundary element method for engineers. London, Pentech Press.

BREBBIA, C. A. \& DOMINGUEZ, J. (1992). Boundary elements - and introductory course. Southampton, Computational Mechanics Publications.

BREBBIA, C. A.; TELLES, J. C. F.; WROBEL, L. C. (1984). Boundary element techniques. Berlin, Springer-Verlag.

CALDERÓN, E. T. (1996). Sobre o uso do método dos elementos de contorno (MEC) para o estudo de interação de placas com o meio contínuo. São Carlos, Tese (Doutorado) - Escola de engenharia de São Carlos, Universidade de São Paulo.

CODA, H. B. (1993). Análise tridimensional transiente de estruturas pela combinação entre o método dos elementos de contorno e o método dos elementos finitos. São Carlos, Tese (Doutorado) - Escola de engenharia de São Carlos, Universidade de São Paulo.

CRUSE, T. A. (1969). Numerical solutions in three dimensional elastostatics. International Journal of Solids and Structures, v. 5, n. 12, pp. 1259-1274. 
DUMONT, N. A. (1999). An assessment of the spectral properties of the matrices obtained in the boundary element methods. In: XV Congresso Brasileiro de Engenharia Mecânica. Águas de Lindóia, São Paulo.

FOLTRAN, C. E. (1999). Análise de problemas planos em regime elasto-plástico pelo método dos elementos de contorno. Campinas, Dissertação (Mestrado) Faculdade de Engenharia Civil, Universidade Estadual de Campinas.

HAMMER, P. C.; MARLOWE, O. J.; STROUD, A. H. (1956). Numerical integration over simplexes and cones. Mathematical Tables and Other Aids to Computation, v. X, n. 55, pp. 130-137.

INGBER, M. S. \& RUDOLPHI, T. J. (1990). Solution of potential problems using combinations of the regular and derivative boundary integral equations. Applied Mathematical Modelling, v. 14, n. 10, pp. 536-543.

LOVE, A. E. H. (1944). A treatise on the mathematical theory of elasticity: $4^{\text {th }}$. ed. New York, Dover Publications.

MOMHA, M. L.; VENTURINI, W. S.; CODA, H. B. (1996). A simple technique to evaluate quasi-singular integrals. In: I Seminário sobre o Método dos Elementos de Contorno. Rio de Janeiro.

RIBEIRO, G. O. (1992). Sobre a formulação do método dos elementos de contorno para a flexão de placas usando as hipóteses de Reissner. São Carlos, Tese (Doutorado) - Escola de engenharia de São Carlos, Universidade de São Paulo.

RIZZO, F. J. (1967). An integral equation approach to boundary value problems of classical elastostatics. Quarterly of Applied Mathematics, v. XXV, pp. 83-95.

RUDOLPHI, T. J. (1989). Higher order elements and element enhancement by combined regular and hypersingular boundary integral equations. In: ANNIGERI, B. S. \& TSENG, K., ed. Boundary element methods in engineering. New York, Springer-Verlag. 
SÁ, P. A. C. O. \& TELLES, J. C. F. (1986). Análise de problemas de elasticidade linear tridimensional pelo método dos elementos de contorno utilizando as soluções fundamentais de Kelvin e Mindlin. In: Congresso Latino Americano Sobre Métodos Computacionais para Engenharia. São Carlos, v. I, pp. 43-60.

SCHIEL, F. (1984). Introdução à resistência dos materiais. São Paulo, Editora Harper \& Row do Brasil Ltda.

TADEU, A. \& ANTÓNIO, J. (2000). Use of constant, linear and quadratic boundary elements in $3 D$ wave diffraction analysis. Engineering Analysis with Boundary Elements, v. 24, pp. 131-144.

TELLES, J. C. F. \& PRADO, A. A. (1993). Hyper-singular formulation for $2 D$ potential problems. In: ALIABADI, M. H. \& BREBBIA, C. A., ed. Advanced Formulations in Boundary Element Methods. Great Britain, The Cromwell Press.

TIMOSHENKO, S. P. \& WOINOWSKY-KRIEGER, S. (1956). Theory of plates and shells. New York, McGraw-Hill.

TIMOSHENKO, S. P. \& GOODIER, J. N. (1970). Theory of elasticity. Tokyo, McGraw-Hill Kogakusha.

TIMOSHENKO, S. P. \& GERE, J. E. (1994). Mecânica dos sólidos. Tradução - José Rodrigues de Carvalho. Rio de Janeiro, Livros Técnicos e Científicos S.A. 


\section{OBRAS CONSULTADAS}

BARBIRATO, J. C. C. (1991). Formulação do método dos elementos de contorno para sólidos elásticos tridimensionais; baseada na solução fundamental de Mindlin. São Carlos, Dissertação (Mestrado) - Escola de engenharia de São Carlos, Universidade de São Paulo.

BEER, G. \& WATSON, J. O. (1994). Introduction to finite and boundary element methods for engineers. West Sussex, John Wiley \& Sons Ltd.

CHATI, M. K., MUKHERJEE, S., MUKHERJEE, Y. X. (1999). The boundary node method for three-dimensional linear elasticity. International Journal for Numerical Methods in Engineering, v. 46, pp. 1163-1184.

CODA, H. B. (1990). Análise da vibração livre de meios elásticos bidimensionais pelo método dos elementos de contorno. São Carlos, Dissertação (Mestrado) Escola de engenharia de São Carlos, Universidade de São Paulo.

CODA, H. B. (2000). Contribuição à análise dinâmica transiente de meios contínuos pelo método dos elementos de contorno. São Carlos, Tese (Livre-docência) Escola de engenharia de São Carlos, Universidade de São Paulo.

CRUSE, T. A. \& RICHARDSON, J. D. (1996). Non-singular Somigliana stress identities in elasticity. International Journal for Numerical Methods in Engineering, v. 39, pp. 3273-3304.

EL-ZAFRANY, A., FADHIL, S., DEBBIH, M. (1994a). A modified Kirchhoff theory for boundary element bending analysis of thin plates. International Journal of Solids and Structures, v. 31, n. 21, pp. 2885-2899. 
EL-ZAFRANY, A., FADHIL, S., DEBBIH, M. (1994b). Boundary element analysis of thick Reissner plates in bending. Engineering Analysis with Boundary Elements, v. 14, n. 2, pp. 159-169.

EL-ZAFRANY, A., FADHIL, S., DEBBIH, M. (1995). An efficient approach for boundary element bending of thin and thick plates. Computers and Structures, v. 56, n. 4 , pp. 565-576.

FADHIL, S. \& EL-ZAFRANY, A. (1994). Boundary element analysis of thick Reissner plates on two-parameter foundation. International Journal of Solids and Structures, v. 31, n. 21, pp. 2901-2917.

HARTMANN, F. (1980). Computation the C-matrix in non-smooth boundary points. In: BREBBIA, C. A., ed. New developments in boundary element methods. Southampton, Computational Mechanics Centre publication.

INGBER, M. S. (1989). The evaluation of the normal derivative along the boundary in the direct boundary element method. Applied Mathematical Modelling, v.13, n.1, pp.32-40.

KARAMI, G. \& DERAKHSHAN, D. (1999). An efficient method to evaluate hypersingular and supersingular integrals in boundary integral equations analysis. Engineering Analysis with Boundary Elements, v. 23, pp. 317-326.

LEUNG, C. Y. \& WALKER, S. P. (1997). Iterative solution of large three-dimensional BEM elastostatics analyses using the GMRES technique. International Journal for Numerical Methods in Engineering, v. 40, pp. 2227-2236.

LIU, Y. (1998). Analysis of shell-like structures by the boundary element method based on 3-D elasticity: Formulation and Verification. International Journal for Numerical Methods in Engineering, v. 41, pp. 541-558.

LUTZ, E., YE, W., MUKHERJEE, S. (1998). Elimination of rigid body modes from discretized boundary integral equations. International Journal of Solids and Structures, v. 35, n. 33, pp. 4427-4436. 
MANSUR, W. J., ARAUJO, F. C., MALAGHINI, J. E. B. (1992). Solution of BEM systems of equations via iterative techniques. International Journal for Numerical Methods in Engineering, v. 33, pp. 1823-1841.

MATSUNAGA, H. (1997). Buckling instabilities of thick elastic plates subjected to inplane stresses. Computers and Structures, v. 62, n. 1, pp. 205-214.

MORAN, K. M. \& CICCI, D. A. (2000). Sensitivity of ridge-type estimation methods to condition number. Applied Mathematics and Computation, v. 112, pp. 143-159.

MUKHERJEE, Y. X., MUKHERJEE, S., SHI, X., NAGARAJAN, A. (1997). The boundary contour method for three-dimensional linear elasticity with a new quadratic boundary element. Engineering Analysis with Boundary Elements, v. 20, pp. 35-44.

PAPADRAKAKIS, M. \& BITOULAS, N. (1993). Accuracy and effectiveness of preconditioned conjugate gradient algorithms for large and ill-conditioned problems. Computers Methods in Applied Mechanics and Engineering, v. 109, pp. 219-232.

RASHED, Y. F., ALIABADI, M. H., BREBBIA, C. A. (1998). Hypersingular boundary element formulation for Reissner plates. International Journal of Solids and Structures, v. 35, n. 18, pp. 2229-2249.

ROCHA, F. S. (1988). Análise de descontinuidades pelo método dos elementos de contorno. São Carlos, Tese (Doutorado) - Escola de engenharia de São Carlos, Universidade de São Paulo.

STERN, M. (1979). A general boundary integral formulation for the numerical solutions of plate bending problems. International Journal of Solids and Structures, v. 15 , n. 10 , pp. $769-782$.

TIMOSHENKO, S. P. (1983). History of strength of materials. New York, McGrawHill Book Co., Inc. 
UREKEW, T. J. \& RENCIS, J. J. (1994). An iterative solution strategy for boundary element equations from mixed boundary value problems. Computers Methods in Applied Mechanics and Engineering, v. 118, pp. 13-28.

VALENTE, F. P. \& PINA, H. L. G. (1998). Iterative solvers for BEM algebraic systems of equation. Engineering Analysis with Boundary Elements, v. 22, pp. 117124.

VENTURINI, W. S. (1983). Boundary element method in geomechanics. Berlin, Springer-Verlag.

YOUNG, A. (1997). Improved numerical method for the traction boundary integral equation by application of stokes' theorem. International Journal for Numerical Methods in Engineering, v. 40, pp. 3141-3161. 


\section{APÊNDICE I}

\section{Soluções analíticas de alguns problemas apresentados}

\section{A. Sólido submetido à força longitudinal}

Supondo um sólido elástico, homogêneo e isotrópico submetido a uma força longitudinal distribuída sobre o topo, e que possui módulo de elasticidade $\mathrm{E}$ e coeficiente de Poisson v, como mostra a figura l.1.

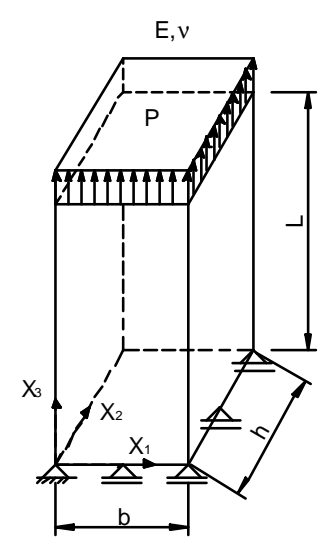

Figura $\quad$ I.1 - Sólido elástico sob força longitudinal

As tensões deste sólido são dadas pelas seguintes expressões:

$$
\left\{\begin{array}{l}
\sigma_{x}=\sigma_{y}=0 \\
\sigma_{z}=P \\
\tau_{x y}=\tau_{x z}=\tau_{y z}=0
\end{array}\right.
$$


E os deslocamentos são dados pelas expressões:

$$
\left\{\begin{array}{l}
u(x, y, z)=-\frac{1}{E} \cdot v \cdot P \cdot x \\
v(x, y, z)=-\frac{1}{E} \cdot v \cdot P \cdot y \\
w(x, y, z)=\frac{1}{E} \cdot P \cdot z
\end{array}\right.
$$

\section{B. Sólido submetido à força transversal}

Supondo o mesmo sólido anterior, só que submetido à força transversal, como mostra a figura I.2, têm-se agora dois tipos de deslocamentos: um devido à flexão e outro devido ao cisalhamento. Este último pode normalmente ser desprezado para valores de $L>6 B$, de acordo com Schiel (1984), pois sua ordem de grandeza é bem menor do que o deslocamento devido àflexão. Mas para se ter o valor mais correto para os deslocamentos, e inclusive calcular sólidos com relação $\mathrm{L}<3 \mathrm{~B}$, como apresentado nos exemplos 2 e 3, apresenta-se neste apêndice o deslocamento total causado pela força transversal.

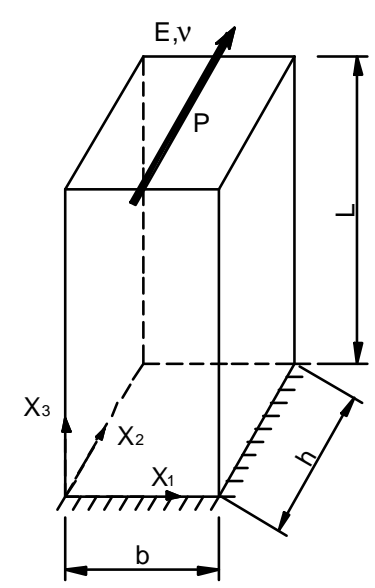

Figura $\quad 1.2$ - Sólido elástico sob força transversal 
As tensões são dadas pelas seguintes expressões:

$$
\left\{\begin{array}{l}
\sigma_{x}=\sigma_{y}=0 \\
\sigma_{z}=\frac{M}{I} \cdot y \\
\tau_{x y}=\tau_{x z}=0 \\
\tau_{y z}=\frac{3}{2} \cdot \frac{V}{b \cdot h} \cdot\left[1-\left(\frac{y}{h / 2}\right)^{2}\right]
\end{array}\right.
$$

Onde:

M: momento fletor na seção em estudo;

V: esforço cortante na seção em estudo;

I: momento de inércia àflexão da seção em estudo;

b e h: dimensões da seção.

O deslocamento transversal para o eixo central devido somente à flexão é dado pela expressão:

$$
v_{1}\left(x_{3}\right)=\frac{P \cdot L^{3}}{6 \cdot E \cdot I} \cdot\left[2-3 \cdot \frac{z}{L}+\left(\frac{z}{L}\right)^{3}\right]
$$

$\mathrm{E}$ o deslocamento transversal devido às tensões de cisalhamento, obtido através do método de energia de deformação é:

$$
v_{2}\left(x_{3}\right)=1,2 \cdot \frac{V \cdot L_{z}}{G \cdot A}
$$

Onde:

V: esforço cortante na seção;

$\mathrm{L}_{z}$ : comprimento do sólido até a seção;

G: módulo de elasticidade transversal;

A: área da seção em estudo.

E o deslocamento total é obtido por:

$$
v=v_{1}+v_{2}
$$




\section{Viga bi-apoiada submetida a uma carga concentrada no meio do vão}

Para o cálculo da viga bi-apoiada, também foi levado em consideração a deformação devido æ̀s tensões cisalhantes. A viga também é um sólido elástico, isotrópico e homogêneo, como mostra a figura I.3.

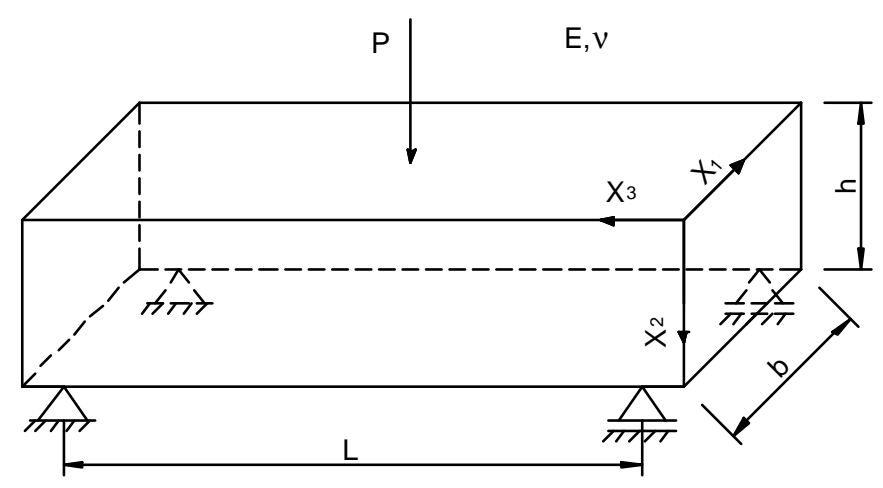

Figura $\quad$ I.3 - Viga bi-apoiada sob flexão simples

As expressões para tensões são análogas às apresentadas para o sólido submetido à força transversal, pois é um caso de flexão simples. O deslocamento transversal devido àflexão sobre o eixo central é o seguinte:

$$
v_{1}\left(x_{3}\right)=\frac{P \cdot L^{3}}{16 \cdot E \cdot I} \cdot\left[\frac{z}{L}-\frac{4}{3} \cdot\left(\frac{z}{L}\right)^{3}\right], \quad p / x_{3} \leq \frac{L}{2}
$$

E o deslocamento devido às tensões cisalhantes é obtido analogamente ao exemplo anterior:

$$
v_{2}\left(x_{3}\right)=1,2 \cdot \frac{V \cdot L_{z}}{G \cdot A}, \quad p / z \leq \frac{L}{2}
$$

Assim o deslocamento total é dado por:

$$
v=v_{1}+v_{2}
$$




\section{Esfera vazada submetida à pressão interna}

Este exemplo trata-se de uma esfera vazada com pressão interna e externa, a figura I.4 mostra a esfera com suas dimensões.

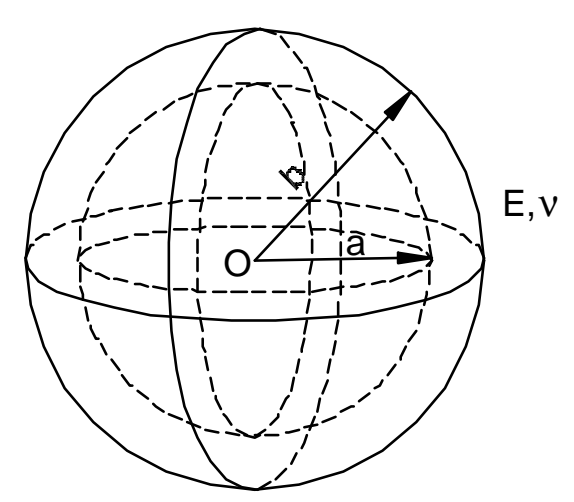

Figura I.4 - Esfera vazada

As tensões em termos de coordenadas esféricas, obtidas a partir de Timoshenko \& Goodier (1970) são:

$$
\left\{\begin{array}{l}
\boldsymbol{\sigma}_{r}=\frac{E}{(1+\mathbf{v}) \cdot(1-2 \cdot \mathbf{v})} \cdot\left[(1-\mathbf{v}) \frac{\partial u}{\partial r}+2 \cdot \boldsymbol{v} \cdot \frac{u}{r}\right] \\
\sigma_{t}=\frac{E}{(1+\mathbf{v}) \cdot(1-2 \cdot \mathbf{v})} \cdot\left(\frac{u}{r}+\mathbf{v} \cdot \frac{\partial u}{\partial r}\right)
\end{array}\right.
$$

Onde $\sigma_{\mathrm{r}}$ é a tensão radial e $\sigma_{\mathrm{t}}$ é a tensão circunferencial. $E$ os deslocamentos radiais são dados por:

$$
u(r)=\frac{(1-2 \cdot \mathrm{v}) \cdot\left(P_{i} \cdot a^{3}-P_{o} \cdot b^{3}\right)}{E \cdot\left(b^{3}-a^{3}\right)} \cdot r+\frac{(1+\mathrm{v}) \cdot\left(P_{i}-P_{o}\right) \cdot a^{3} \cdot b^{3}}{2 \cdot E \cdot\left(b^{3}-a^{3}\right)} \cdot \frac{1}{r^{2}}
$$

Onde:

$P_{\mathrm{i}}$ : pressão interna;

$P_{0}$ : pressão externa;

a: raio interno;

b: raio externo;

r: raio do ponto escolhido. 